Portland State University

PDXScholar

Winter 3-14-2014

\title{
The Design, Implementation, Assessment, and Evaluation of a Power Systems Protection Laboratory Curriculum
}

Jennifer Ferris

Portland State University

Follow this and additional works at: https://pdxscholar.library.pdx.edu/open_access_etds

Part of the Curriculum and Instruction Commons, and the Power and Energy Commons Let us know how access to this document benefits you.

\section{Recommended Citation}

Ferris, Jennifer, "The Design, Implementation, Assessment, and Evaluation of a Power Systems Protection Laboratory Curriculum" (2014). Dissertations and Theses. Paper 1623.

https://doi.org/10.15760/etd.1622

This Thesis is brought to you for free and open access. It has been accepted for inclusion in Dissertations and Theses by an authorized administrator of PDXScholar. Please contact us if we can make this document more accessible: pdxscholar@pdx.edu. 
The Design, Implementation, Assessment, and Evaluation of a Power Systems Protection Laboratory Curriculum

by

Jennifer Beth Ferris

A thesis submitted in partial fulfillment of the requirements for the degree of

\author{
Master of Science \\ in \\ Electrical and Computer Engineering
}

Thesis Committee:

Dr. Robert Bass, PhD, Chair

Dr. Melinda Holtzman

Dr. James Morris

Portland State University

2014 
C 2014 Jennifer Beth Ferris 


\begin{abstract}
Over the last the last three decades, there has been a significant and documented decrease of power engineering curriculum within U.S. electrical engineering programs.

Development of lifelong learners capable of critical and independent thinking is required to replace the large number of upcoming retirees from the industry and to prepare for the engineering challenges new technologies, laws and regulations are bringing to the electrical power system. In response to these challenges, Portland State University redesigned its BSEE and MSECE power engineering programs in order to create a launching pad for successful power engineering careers. A course series on power system protection is part of the requisite curriculum for both programs. Due to the complex and applied nature of this subject, the course features a laboratory component.
\end{abstract}

This thesis addresses the pedagogical and engineering problems associated with developing power systems protection laboratory curriculum. Laboratory equipment and curriculum design were guided by outcomes defined by ABET EC2000 and specific outcomes defined for the laboratory that we adopted from research commissioned by ABET. Hands-on experience with industry equipment and software enhances classroombased course curriculum, expanding student understanding of the complexities of the subject of power system protection. Intergroup and intragroup communication is required in the laboratory, as are weekly written reports synthesizing subject material and experimental results. The outcomes of the laboratory are evaluated through a combination of grading rubrics and student participation in individual laboratory 
assignment surveys.

The laboratory is comprised of three stations consisting of electromechanical (EM) relays and digital relay equipment. The practical experience with industry standard relays types happens in concert with a software-based simulation program. There are three phases of development; Phase I includes the design of the physical laboratory and the procural, or design and fabrication, of necessary equipment. Phase II involves the teaching term of the laboratory and the active assessment. Phase III involves the design and specification of a model-scale laboratory, to be built in future terms, which will provide students with the ability to test power system protection equipment in a physically simulated environment. This model-scale system will also provide a platform for future expansions of the power system protection laboratory in terms of distance protection, generation control, phasor measurement units, and system control schemes. 
This work is dedicated to my students. It was done for you and could not have been done without you. Thank you.

That which I begin with enthusiasm, may I end with awareness 


\section{Acknowledgments}

First and foremost I thank my parents for raising me so carefully and with such loving support, always encouraging my continuous pursuit of higher education. You both are undoubtedly a huge part of the reason I find academia so incredibly extraordinary and rewarding. I thank Bop Bop and Grandma Me for the foundations they set for me. A big thank you to Kelly for his friendship, inspiration, and enthusiasm for my work. Thank you to Ashely for being a cohort and friend. Also, thank you to Alison and Ferris for being there the whole way through; and to Mattie for infusing some brief, beautifully human magic to the winter term by providing me a space to be soft and just myself, outside of my research.

I thank my committee members for their participation in this process. I thank SEL for their generous donation of our digital relays, which got the lab off the ground, as well as for their continued involvement expanding our laboratory equipment. I also thank AccuSource, Tektronix, Test Equity, and PGE for their contributions to the laboratory equipment. I thank Luis for his experience and for his catalytic direction.

Most of all, my deepest gratitude to Bob for tailoring this research specifically to my academic interests and for providing me with this opportunity to work, learn, build, and develop. Your mentorship has been invaluable; not only because of your direct guidance but because you lead by example, effectively blending engineering and education. Thank you for being a role model and for your unwavering confidence in my ability to succeed. 


\section{Table of Contents}

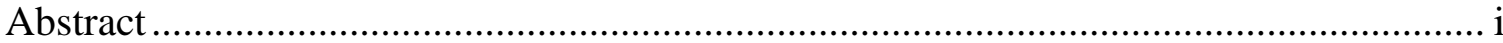

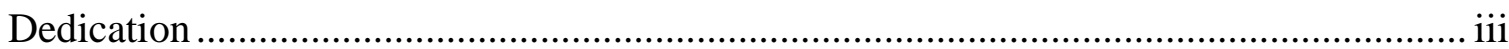

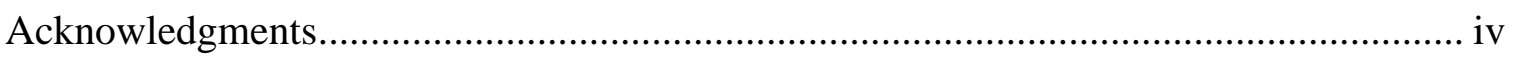

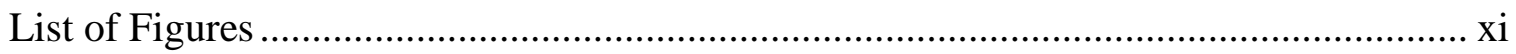

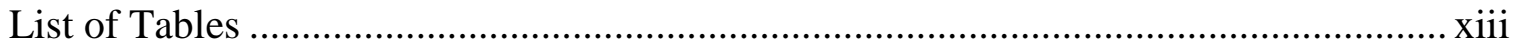

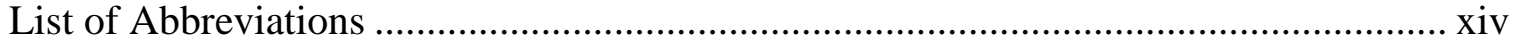

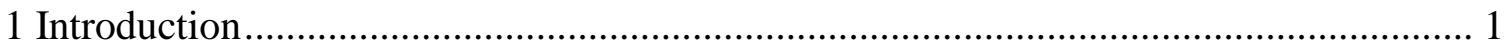

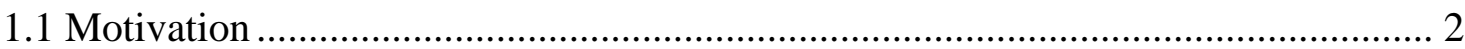

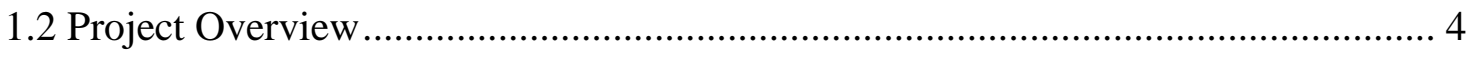

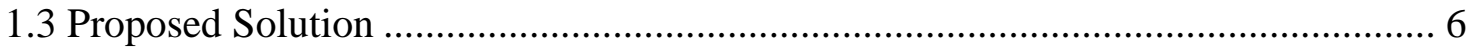

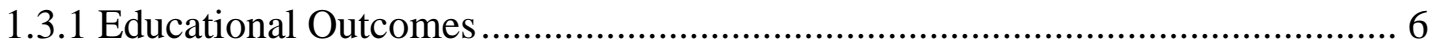

1.3.2 Student and Laboratory Outcomes ............................................................. 7

1.4 Laboratory Development Phases................................................................... 9

1.4.1 Laboratory Development Phases I........................................................... 10

1.4.2 Laboratory Development Phases II .......................................................... 10

1.4.3 Laboratory Development Phases III ........................................................... 11

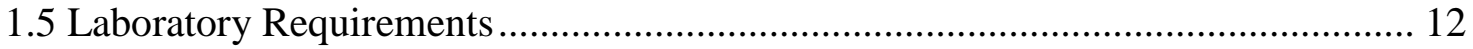

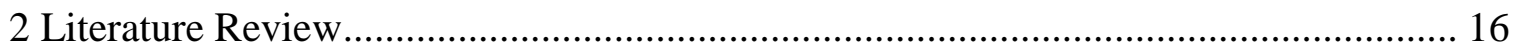

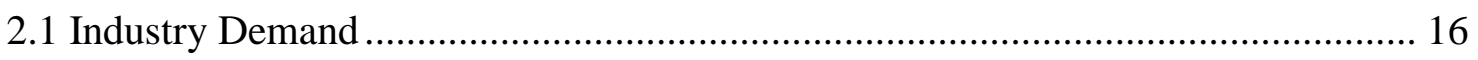

2.2 The Laboratory Contribution to Engineering Education.................................... 18

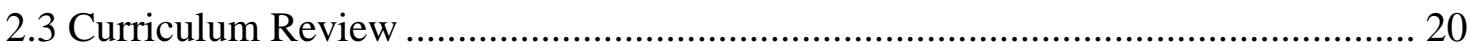

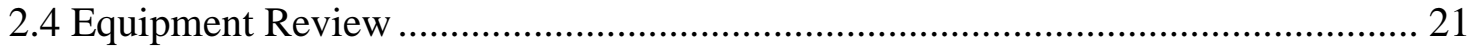

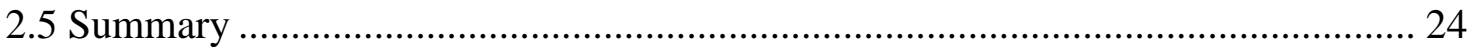

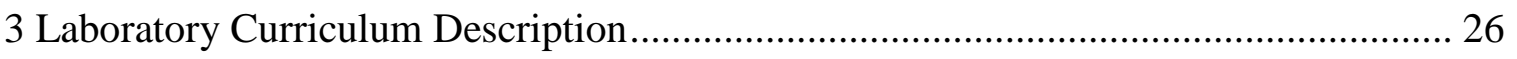

3.1 Lab 1: Introduction to ASPEN software ............................................................ 26

3.2 Lab 2: Thermal properties of a conductor in free air ........................................... 28

3.3 Lab 3: Auxiliary Electromechanical (EM) Relays .............................................. 32

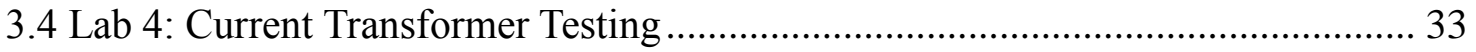

3.5 Lab 5: Electromechanical Overcurrent Relays.................................................. 38

3.6 Lab 6: ASPEN radial OC coordination .......................................................... 42 
3.7 Lab 7: Digital relay (SEL-551) testing (50/51) .................................................... 44

3.8 Lab 8: ASPEN looped system coordination............................................................ 46

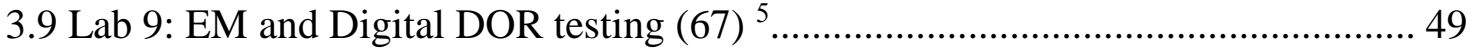

4 Equipment Design............................................................................................... 56

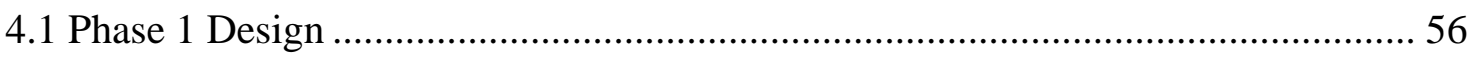

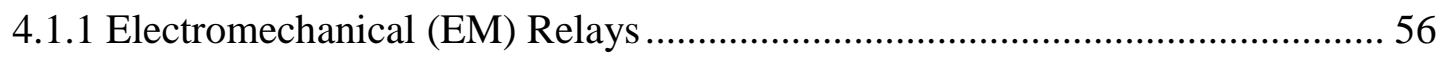

4.1.2 Fuse Holder......................................................................................... 58

4.1.3 Electromechanical Relay Testing Systems and High Current Source.............. 59

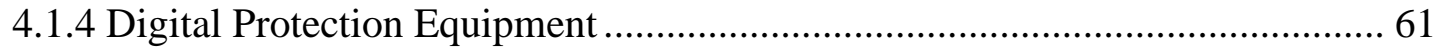

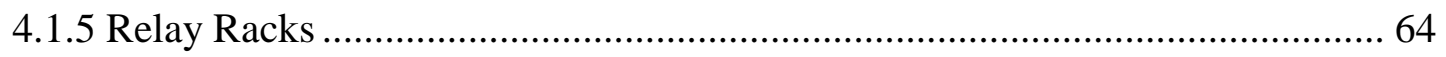

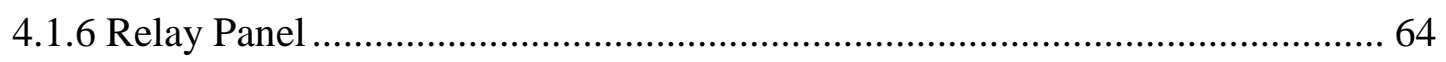

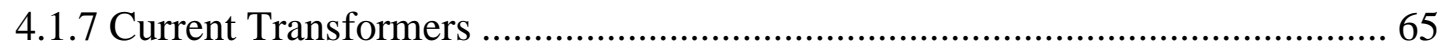

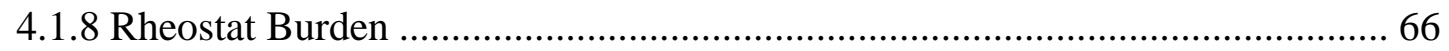

4.1.9 Oscilloscopes and Current Probes ................................................................... 67

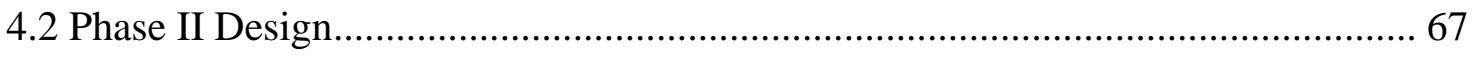

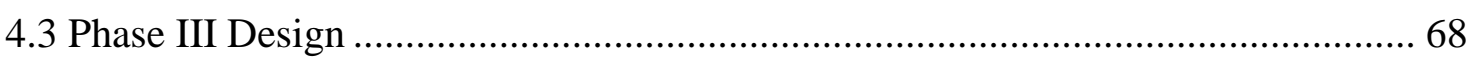

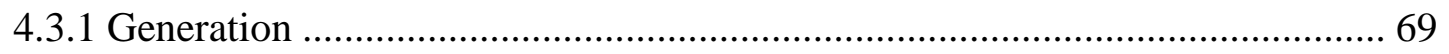

4.3.2 Generation Transformers ............................................................................. 71

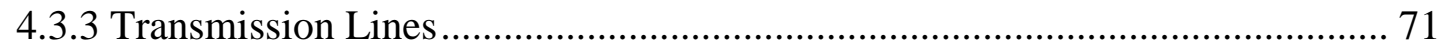

4.3.4 Load Shifting Transformer Bank............................................................... 74

4.3.5 Bus, Generation, and Load Panels............................................................. 76

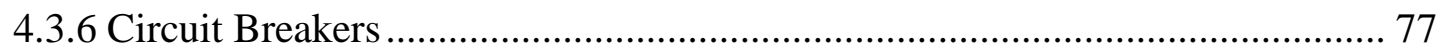

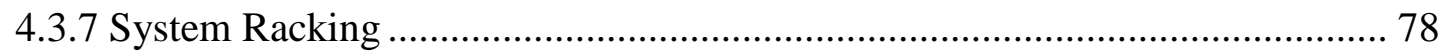

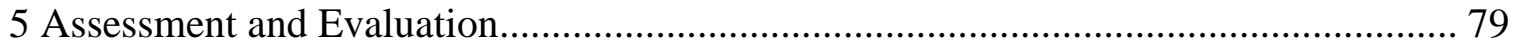

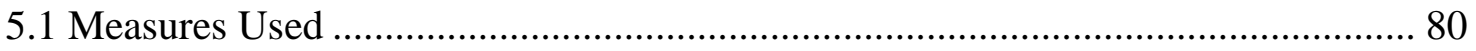

5.1.1 Rubric Assessment of Weekly Reports .......................................................... 80

5.1.2 Assignment Outcomes and Overall Laboratory Outcomes Surveys ................ 81

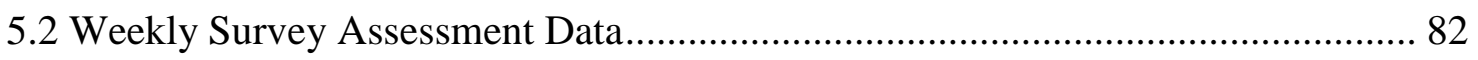

5.2.1 Laboratory 1 - ASPEN Introduction Survey Results........................................ 82

5.2.2 Laboratory 2 - Thermal Properties Survey Results ........................................... 86 
5.2.3 Laboratory 3 - Auxiliary Relay Testing Survey Results .................................. 90

5.2.4 Laboratory 4 - Current Transformer Testing Survey Results ............................. 94

5.2.5 Laboratory 5 - Electromechanical OC Testing Survey Results......................... 97

5.2.6 Laboratory 6 - ASPEN Radial OC Coordination Survey Results ................... 101

5.2.7 Laboratory 7 - Digital OC Relay Testing Survey Results ................................ 104

5.2.8 Laboratory 8 - ASPEN Looped OC Coordination Survey Results ................ 108

5.2.9 Analysis of Laboratory 9 - Directional Over-current Relays Survey Results ......

5.3 Overall Laboratory Outcomes Assessment Data ................................................. 114

5.3.1 Feisel's Laboratory Outcomes Survey Results................................................. 114

5.3.2 Laboratory as a separate, one credit course .................................................... 119

5.3.3 Overall Laboratory Student Comments ……………………......................... 120

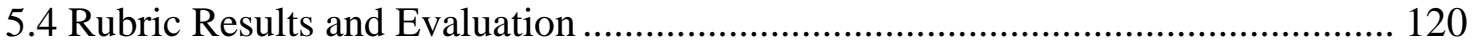

5.5 Closing the Loop - Improvements to the Laboratory Based on Assessments....... 123

5.5.1 Survey Results for Laboratory Assignment and Feisel Outcomes ................. 123

5.5.2 Rubric Results for ABET Student Learning Outcomes.................................... 130

5.6 Evaluation - Outcomes and lessons learned .................................................... 130

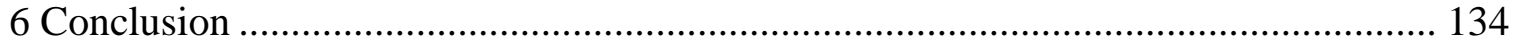

6.1 Engineering Solution...................................................................................... 134

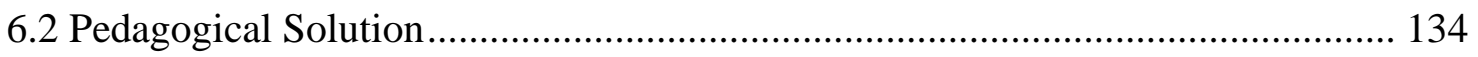

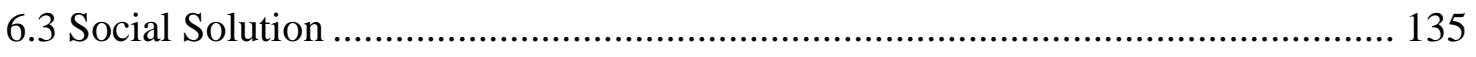

Appendix A: Revised Laboratory Assignment Instructions ........................................... 141

A.1 Revised ASPEN Software Introduction Instructions ............................................ 142

A.2 Revised Wire Heating and Fuses Instructions..................................................... 153

A.3 Revised Auxiliary Relay Testing and CT Testing Instructions............................. 156

A.4 Revised EM Relay Radial Over-current Protection (50/51) Instructions.............. 163

A.5 Revised Coordination of OC Relays in Radial Systems Instructions .................. 167

A.6 Revised Digital Relay Radial Over-current Protection (50/51) Instructions ....... 170

A.7 Revised Protection Coordination of Looped Systems Instructions ...................... 174

A.8 Revised Directional Over-current Relays (67) Instructions .................................. 178

Appendix B: Original Assignment Instructions......................................................... 187 
B.1 Original ASPEN Software Introduction Instructions ................................... 188

B.2 Original Wire Heating and Fuses Instructions ............................................. 192

B.3 Original Auxiliary Relay Testing Instructions ................................................. 195

B.4 Original Current Transformer Testing Instructions ........................................ 200

B.5 Original EM Relay Radial Over-current Protection (50/51) Instructions ............ 203

B.6 Original Coordination of OC Relays in Radial Systems Instructions ................ 207

B.7 Original Digital Relay Radial Over-current Protection (50/51) Instructions ....... 210

B.8 Original Protection Coordination of Looped Systems Instructions .................... 213

B.9 Original Directional Over-current Relays (67) Instructions............................. 216

Appendix C: Phase I - Laboratory Design Drawings............................................... 224

C.1 Protection relay panel and rack plan view ................................................... 225

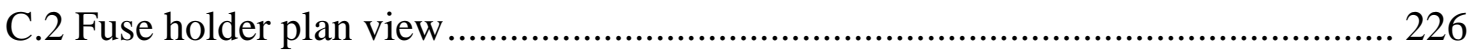

Appendix D: Phase III - Laboratory-Scale Power System Design Drawings ................ 227

D.1 Phase III model-scale power system one-line drawing ................................... 228

D.2 Phase III model-scale power system three-phase line drawing......................... 229

D.3 Phase III model-scale power system Bus 1 three-phase wiring diagram ............ 230

D.4 Phase III model-scale power system Bus 2 three-phase wiring diagram ............ 231

D.5 Phase III model-scale power system Bus 3 three-phase wiring diagram ............ 232

D.6 Phase III model-scale power system generation transformer bank ..................... 233

D.7 Phase III model-scale power system circuit breaker control circuit diagram ...... 234

D.8 Phase III model-scale power system load shifting transformer bank................. 235

D.9 Phase III model-scale power system generic fault simulator wiring diagram .... 236

D.10 Phase III model-scale power system Bus 1 plan drawing .............................. 237

D.11 Phase III model-scale power system Bus 2 plan drawing ............................... 238

D.12 Phase III model-scale power system Bus 3 plan drawing ............................... 239

D.13 Phase III model-scale power system load shifting transformer plan drawing ... 240

D.14 Phase III model-scale power system transmission line module plan drawing ... 241

D.15 Phase III model-scale power system 10 mile transmission line panel drawing . 242

D.16 Phase III model-scale power system 8 mile transmission line panel drawing ... 243

D.17 Phase III model-scale power system 6 mile transmission line panel drawing ... 244 
D.18 Phase III model-scale power system Generation 1 panel drawing .................... 245

D.19 Phase III model-scale power system Generation 2 panel drawing ................... 246

D.20 Phase III model-scale power system WECC panel drawing ............................ 247

D.21 Phase III model-scale power system Loads panel drawing ............................ 248

D.22 Phase III model-scale power system From 10 Mile Line panel drawing .......... 249

D.23 Phase III model-scale power system To 10 Mile Line panel drawing .............. 250

D.24 Phase III model-scale power system From 8 Mile Line panel drawing ............ 251

D.25 Phase III model-scale power system To 8 Mile Line panel drawing ................. 252

D.26 Phase III model-scale power system From 6 Mile Line panel drawing ............. 253

D.27 Phase III model-scale power system To 6 Mile Line panel drawing ................ 254

D.28 Phase III model-scale power system Sync Check panel drawing .................... 255

Appendix E: Laboratory Assignment Rubrics ...................................................... 256

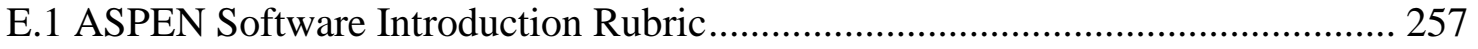

E.2 Wire Heating and Fuses Rubric ................................................................. 259

E.3 Auxiliary Relay Testing Rubric ........................................................... 261

E.4 Current Transformer Testing Rubric ........................................................... 263

E.5 EM Relay Radial Over-current Protection (50/51) Rubric ................................ 265

E.6 Coordination of OC Relays in Radial Systems Rubric................................... 267

E.7 Digital Relay Radial Over-current Protection (50/51) Rubric............................ 269

E.8 ASPEN Looped System Coordination Rubric ............................................. 271

E.9 Directional Over-current Relays (67) Rubric ................................................ 273

Appendix F: Laboratory Course Syllabus............................................................. 275

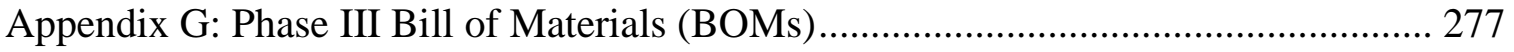

G.1 BOM 1 - General panel materials list for drawings C.1 - C.14 ....................... 278

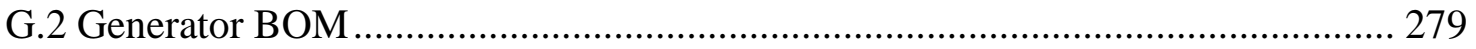

G.3 Transmission line module BOM ........................................................ 280

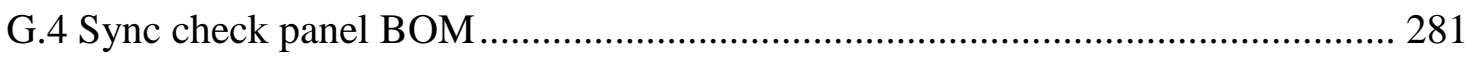

G.5 Electromechanical relays and relay testers BOM...................................... 282

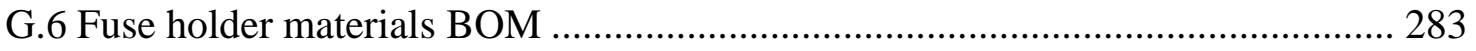

G.7 Current Transformers and rheostat BOM.................................................. 284 
Appendix F: Student Survey Comments............................................................ 285 


\section{List of Figures}

Figure 1: Laboratory 1 - an example of a radial system model in the ASPEN modeling software package.

Figure 2: Laboratory 1 - an example of a looped system model from the Blackburn text ..

Figure 3: Thermal properties experiment: physical implementation of wire being tested with a high current source connection and thermocouple measuring temperature. Acrylic fuse holder was designed

Figure 4: Comparison of experimental to theoretical $\mathrm{T}(\mathrm{t})$ curve. Expand more. Describe the purpose of this plot, with respect to the curriculum................................................ 30

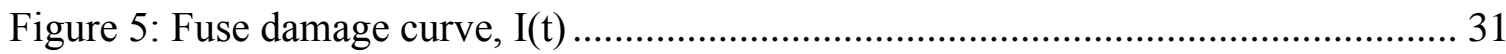

Figure 6: Generic plunger style auxiliary relay diagram ............................................ 33

Figure 7: CT magnetization testing connection diagram............................................... 34

Figure 8: Magnetization curve of AL500 Veris CT with turns ratio 50:5 ....................... 35

Figure 9: Measurement of a CT to $120 \%$ over nominal primary current at rated burden ....

Figure 10: Measurement of a CT to $300 \%$ over rated burden at nominal primary current ..

Figure 11: Physical setup for $\mathrm{CT}$ testing using the MultiAmp SR-51A relay testing unit....

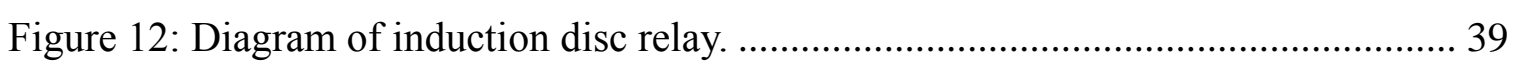

Figure 13: US Standard U3 very inverse curve from the General Electric IAC53

instruction manual. Source: General Electric ……………........................................... 40

Figure 14: US Standard U4 extremely inverse curve from the General Electric IAC77 instruction manual. Source: General Electric …………........................................... 41

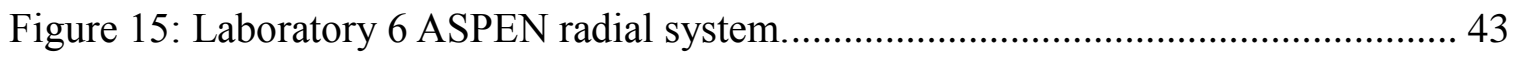

Figure 16: SEL-551 digital overcurrent relay. .......................................................... 45

Figure 17: SEL-551 inverse time OC element testing results.......................................... 45

Figure 18: A three-bus looped power system modeled in ASPEN software for the ninth laboratory assignment covering looped system coordination design .............................. 47

Figure 19: General Electric JBC51N US Standard U2 inverse curve. ............................ 50 Figure 20: General Electric JBC51N overcurrent relay testing input connections diagram.

Figure 21: General Electric JBC51M (-) Y1A relay tesing connections. ......................... 52

Figure 22: Fuse holder design. Source: Ferris ......................................................... 58

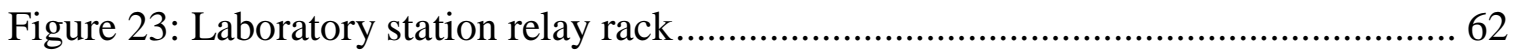

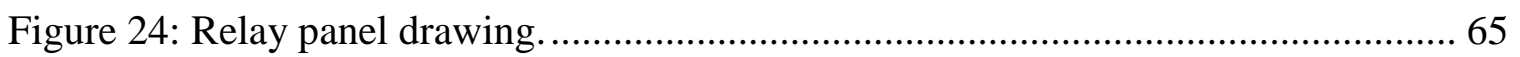




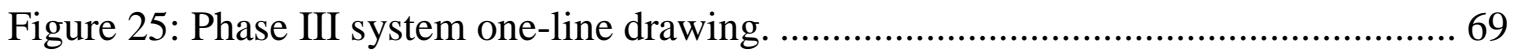

Figure 26: Generation transformer bank drawing..................................................... 70

Figure 27: Transmission module plan drawing. ........................................................ 72

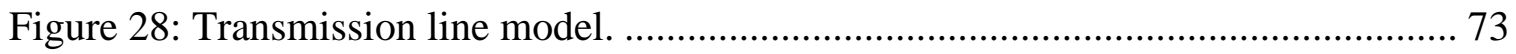

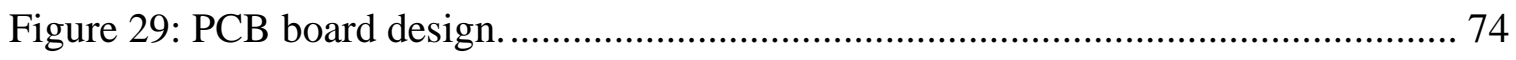

Figure 30: Load shifting transformer bank wiring diagram....................................... 75

Figure 31: Load shifting transformer bank plan drawing. ............................................ 75

Figure 32: Assessment and Evaluation Feedback Cycle. Source: Ferris........................ 79

Figure 33: ASPEN Introduction Survey Results .................................................... 85

Figure 34: Thermal Properties Survey Results ........................................................ 89

Figure 35: Auxiliary Relay Testing Objective 1 Survey Results.................................... 92

Figure 36: Auxiliary Relay Testing Objectives 2 \& 3 Survey Results ........................... 92

Figure 37: Current Transformer Testing Survey Results............................................. 96

Figure 38: EM Relay Radial Over-current Protection Survey Results ......................... 100

Figure 39: ASPEN Coordination of OC Relays in Radial Systems Survey Results ...... 103

Figure 40: Digital Relay Radial Over-current Protection Survey Results ..................... 107

Figure 41: ASPEN Looped OC Coordination Survey Results ................................... 110

Figure 42: Directional Over-current Relays (67) Survey .......................................... 113

Figure 43: Feisel's Laboratory Objectives Survey Results........................................ 118

Figure 44: Grading rubric assessment data ......................................................... 122

Figure 45: Pulsar relay testing unit user manual instructions for directional unit test ... 129

Figure 46: JBC manual relay connections for 3-single phase JBC relays ..................... 179

Figure 47: JBC manual, referenced in Pulsar test instructions, for directional unit test

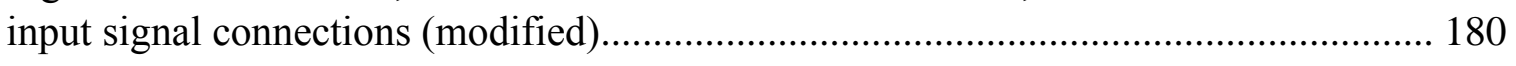

Figure 48: Pulsar manual testing instructions for the directional unit, with noted adjustments for the laboratory testing process ....................................................... 181

Figure 49: US Inverse Curve U2 from JBC51N instruction manual ........................... 182 


\section{List of Tables}

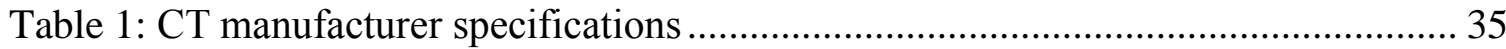

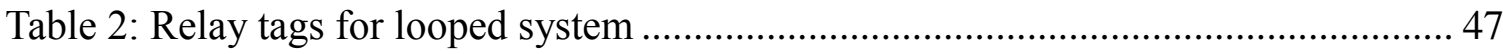

Table 3: Current flow direction for lagging current angles........................................... 51

Table 4: Case 1 fault parameters ............................................................................... 54

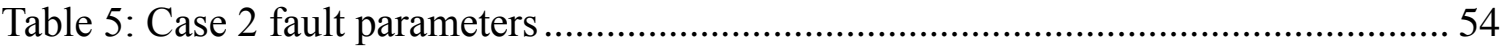

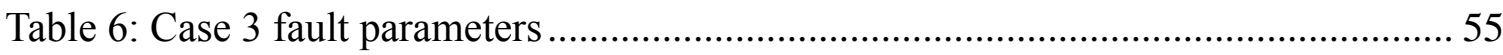

Table 7: Current Workforce \& Eligible for Retirement - 2015 \& Projected to Retire -

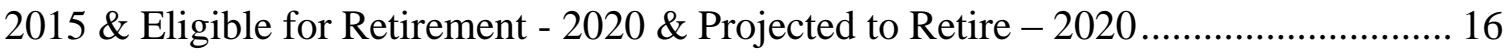

Table 8: Transmission line module impedance parameter values .................................. 73 


$\begin{array}{ll}\text { List of Abbreviations } \\ 50 / 51 & \text { ANSI device number for instantaneous overcurrent/time-de } \\ & \text { overcurrent elements } \\ 67 & \text { ANSI device number for directional overcurrent element } \\ 79 & \text { ANSI device number for circuit breaker re-closer element } \\ \text { DO } & \text { directional overcurrent } \\ \text { DOR } & \text { directional overcurrent relay } \\ \text { EC2000 } & \text { Engineering Criteria 2000 } \\ \text { EM } & \text { multiple of pick-up current } \\ \text { M } & \text { microprocessor } \\ \text { MP } & \text { overcurrent } \\ \text { OC } & \text { Schweitzer Engineering Laboratories } \\ \text { SEL } & \end{array}$




\section{Introduction}

For several decades, the recognition for the need of power engineering focused graduates has been acknowledged in the educational and industrial sectors. [1] [2] At the same time there has been a national decline in engineering program curriculum [3] and power engineering is no exception. With recent publications projecting nation-wide, large-scale retirements from the power industry, [4] [5] the redevelopment of the power engineering curriculum has gained popularity in electrical engineering programs. [6] In response to the need for power engineers entering the workplace, [7] [8] Portland State University has invested in redesigning the three phase power program within the Electrical \& Computer Engineering College that is customized to the large and diverse power industry of the Portland metropolitan area. The research presented in this body of work focuses on the development, execution, assessment, and evaluation of the educational goals of the teaching laboratory for the 400/500-level power system protection course, which is a requisite for all graduates in the power systems emphasis. These educational goals are derived from the standard ABET EC2000 [9] [10] and ABET laboratory objectives, set out by Feisel [11] [12], to provide students with hands-on experience with industry protection equipment and software, enhance the classroom-based course curriculum, and acquaint students with industry standards and design practices. To physically manifest these goals, relevant laboratory equipment is essential to support the curriculum effectively. The procural of necessary equipment specific to this course sequence was achieved thru specification and designs, fabrication, donations through industry partnerships, and purchasing though equipment vendors. 


\subsection{Motivation}

The Portland, OR, metropolitan area is graced with an abundance of power-related entities, creating a strong industry power industry focus. These entities include two federal entities focused on hydropower and transmission, two investor-owned utilities, two dozen power engineering consultancies, several power plant developers \& operators, and a growing number of high-tech manufacturers and software companies focused on smart-grid products and services. [13] We refer to this collection of power-related entities as the Portland Power Pool.

The Portland Power Pool represent a sizable fraction of the regional economy. [13] In previous years, investment in new generation and transmission, innovations in communications and IT, and rapidly-decreasing prices for renewable resources are all contributed to the industry's growth. Now, on the cusp of the pending large-scale retirements, it is necessary for industry-ready power engineering graduates to be entering the workforce who have an education focused on the specific needs and challenges inherent to the Pacific Northwest power industry demands.

In response to these opportunities and challenges, the ECE Department at Portland State University (PSU) has committed to developing educational pathways for electrical engineering students to become power engineers. Two educational pathways are available, leading to BS EE or MS ECE degrees with specializations in power engineering and an overlapping set of three 400/500-level courses has been created in 
order to encourage the BS EE graduates to matriculate into the MS ECE power program. The goal is to attract new students who wish to enhance their educational depth in power engineering while enhancing professional opportunities for PSU graduates. Educational pathways are provided for working professionals to develop a locally-educated engineering workforce supported by the regional power industry. [3] [14] [15] [16] The power system protection laboratory that is the focus of this research is a critical feature of these two programs, developed in tandem with the required power systems protection course, ECE 4/548, to provide practical experience to support the theoretical concepts presented in lecture theory.

In order to provide students with practical hands-on experiences in preparation for careers in the local power industry, we have designed the protection lab curriculum around using standard industry relays, software and test equipment. [12] [17] The purpose of the protection lab is to provide practical educational experiences for both working professionals enhancing their engineering education, and more traditional full-time electrical engineering students. [3] [13]

To establish the role of the laboratory within this engineering program, the educational goals of the lab were derived from ABET EC $2000^{1}$ and the laboratory objectives set out by Feisel. [11] [12] These outcomes provide the foundation to develop, assess, and evaluate the hands-on experience of the student with industry protection equipment and

\footnotetext{
${ }^{1}$ ABET, http://www.abet.org/
} 
software, enhance the classroom-based course curriculum, and acquaint students with industry standards and design practices. [2] [17] [18] The importance of the role in facilitating inductive learning [19] [20] and generating student engagement and enthusiasm in course material is also considered in the ability to retain program enrollments and knowledge retention. [21] [22] [23]

Power system protection is a rich and dense subject, and at PSU the course topics are covered during a fast-paced, ten week lecture series; time spent conducting laboratory experiments is therefore very important for emphasizing the real-world application of lecture material. The protection laboratory curriculum provides students an opportunity to apply theory learned in lecture to practical, industry-relevant issues, in turn preparing students for immediate employment in the electric power industry. [24]

\subsection{Project Overview}

With a shortage of industry-ready engineers entering the workplace, as described by Sen and Grice [4] [5], education of power system theory and application for engineering graduates requires a practical component. [17] [25] In order to address the pedagogical dissonance between theory and practice, the laboratory component of engineering education is vital because it allows for an applied educational bridge to explore industry by utilizing industry standard equipment [3] [26] and through the technique of personal engagement by the students. The effect of this personal involvement in the laboratory cannot be not underestimated in the role the laboratory plays in education. [21] [22] This is partly because curriculum uses industry standard equipment and software contribution 
to the preparation of creating workforce-ready professionals at the time of graduation. [3] [27] [28]

Power systems protection is a very specialized topic within power engineering, yet it is a very essential part of the industry since the reliability of the electric grid itself depends on the safe operation of the system components. [24] [29] The PSU power system protection course topics are covered during a fast-paced, ten week lecture series; time spent conducting laboratory experiments is therefore very important for emphasizing the real-world application of lecture. The protection laboratory curriculum provides students an opportunity to apply theory learned in lecture to practical, industry-relevant issues, in turn preparing students for immediate employment in the electric power industry. Basic theories of equipment damage, system sensors, and different types of overcurrent protection are explored in this laboratory. [24] [29] To succeed in the protection industry, students must have retained a basic knowledge of the relationship of current to time regarding equipment damage, the understanding of the principles of overcurrent (OC) protection, the concepts of relay coordination in different types of systems, and the proper application and setting of industry standard equipment. In the power industry, the outmoded electromechanical (EM) equipment still bears relevancy due to its prevalence on the existing electrical grid and it basic operating principles being the foundations of the modern, microprocessor (MP) based equipment. The outmoded equipment allows the students to physically observe these operating principles, which is not possible with the digital equipment. 


\subsection{Proposed Solution}

Using the adopted educational outcomes from ABET and Feisel, a laboratory course was designed and implemented during the winter term of 2012. This laboratory consists of nine assignments tailored closely to lecture course material and taught over a ten week term. The following subsections detail these outcomes and their purpose in this research.

\subsubsection{Educational Outcomes}

The main goal for the outcome of this laboratory is to provide students with practical, hands-on experience applying concepts learned in lecture to standard industry protection equipment, thereby further enhancing the students' learning and career preparation. To meet this objective, the laboratory outcomes were adopted from the results of the colloquy of engineering educators, and subsequently authored by Feisel, et al, who met in San Diego in 2002 to define "The Fundamental Objectives of Engineering Instructional Laboratories". [11] [12] Developed for ABET with funding from the Sloan Foundation, we adopted a subset of these fourteen published "objectives" when designing the PSU power system protection laboratory curriculum. While defined by Feisel as “objectives”, we have chosen to adopt the ABET definitions of "objectives" and "outcomes", whereby "objectives" are defined as "broad statements that describe what graduates are expected to attain within a few years of graduation. Program educational objectives are based on the needs of the program's constituencies." "Student learning outcomes," aka SLOs, are

${ }^{2}$ Assessment Planning, American Board of Engineering and Technology, http://www.abet.org/assessmentplanning/ 
defined as "describe what students are expected to know and be able to do by the time of graduation. These relate to the knowledge, skills, and behaviors that students acquire as they progress through the program." ${ }^{2}$ As such, we consider the Feisel "objectives" to be "outcomes," and use this terminology hereafter.

In 2009, the IEEE Power System Relaying Committee (PSRC) established goals for protection laboratory curriculum. [24] Following the goal of the PSRC for universities to adopt an education model to successfully prepare students for industry, we established laboratory outcomes for the protection laboratory which will assist in creating a smooth transition from education to industry employment. [11] [12] [24]

\subsubsection{Student and Laboratory Outcomes}

\subsubsection{Laboratory Outcomes Derived from ABET SLOs}

Since 2001 ABET has standardized an outcomes-based accreditation criteria for national engineering programs nationally. [9] These outcomes have been specified as Engineering Criteria 2000 (EC2000), commonly termed ABET Student Outcomes a-k, and are detailed as $^{3}$,

a. an ability to apply knowledge of mathematics, science and engineering;

b. an ability to design and conduct experiments, as well as to analyze and interpret data;

\footnotetext{
${ }^{3}$ ABET, http://www.abet.org/
} 
c. an ability to design a system, component, or process to meet desired needs within realistic constraints such as economic, environmental, social, political, ethical, health and safety, manufacturability, and sustainability;

d. an ability to function on multidisciplinary teams;

e. an ability to identify, formulate, and solve engineering problems;

f. an understanding of professional and ethical responsibility;

g. an ability to communicate effectively;

h. the broad education necessary to understand the impact of engineering solutions in a global, economic, environmental, and societal context;

i. a recognition of the need for, and an ability to engage in life-long learning;

j. a knowledge of contemporary issues;

k. an ability to use the techniques, skills, and modern engineering tools necessary for engineering practice.

For this laboratory focuses on four of these outcomes, a, b, d, and g. These were assessed by weekly report grading rubrics to qualitatively evaluate the students' ability to understand and apply their power system protection engineering knowledge by utilizing the mathematics and engineering taught in lecture theory, the design and analysis of protection coordination schemes, work successfully in teams, and communicate effectively in writing.

\subsubsection{Laboratory Outcomes Derived from Feisel}

For a laboratory to have a functional place within the engineering education paradigm, specific goals, or outcomes, must be established in order for the laboratory to be 
effective. These outcomes then serve as benchmarks for evaluation of course effectiveness. [9] [12] [27] Of the thirteen laboratory outcomes outlined by Feisel [11] [12], we focused on ten specifically for this laboratory,

- Instrumentation (1)

- Models (2)

- Experiment (3)

- Data Analysis (4)

- Design (5)

- Learn from Failure (6)

- Psychomotor (8)

- Safety (9)

- Communication (10)

- Teamwork (11)

The three Feisel outcomes that not adopted for this research were Creativity (7), Ethics in the Laboratory (12), and Sensory Awareness (13).

\subsection{Laboratory Development Phases}

The laboratory is taught during a ten week term in tandem with the course lecture. The presentation of laboratory curriculum aligns closely with the presentation of lecture course topics. [25] 


\subsubsection{Laboratory Development Phases I}

This laboratory was organized to supplement and reinforce the lecture course material by following behind a week after course material was presented. The nine labs each correlate with the previous week's lecture material, with the intention of creating student engagement in the learning process so as to develop greater memory retention of, and general positive attitude toward, the course material. [20] [22]

The initial design of the curriculum and corresponding equipment was considered Phase I of the laboratory. During this time individual laboratory assignments were created based on the suggested outline of the lecture course professor, and rubrics were created for quantitative assessment of the ABET student outcomes. Industry standard equipment and software were specified, ordered and assembled. Equipment donations from Schweitzer Engineering Laboratories (SEL), Test Equity, PGE, PacifiCorp and Veris Industries were secured, and other equipment was purchased with funds granted by the PGE Education Foundation and the PSU Dean's Office. Three laboratory stations were designed and assembled for use. Educational discounts for oscilloscopes were provided by Test Equity and by AccuSource for the digital relay testing unit.

\subsubsection{Laboratory Development Phases II}

Phase II corresponds with the teaching implementation of laboratory during Winter Term 2013. Six three-hour laboratory sessions were conducted weekly to accommodate the large class size and limited number of laboratory stations. During this phase rubrics 
were used to assess ABET outcomes. The individual laboratory assignment objectives were assessed by weekly surveys which involved voluntary student participation. A final student survey was administered at the end of the term to assess the ten laboratory outcomes adopted from Feisel. Adjustments to assignment curriculum were made during this time as well, to accommodate for equipment needs and lecture instructor request for a dedicated current transformer (CT) laboratory prior to the experimentation on overcurrent $(\mathrm{OC})$ relay equipment. The addition of the digital relay testing unit was added at this time after it was discovered that the electromechanical based relay testers did not have the functionality for testing directional current, an important aspect of looped system protection.

\subsubsection{Laboratory Development Phases III}

The final phase of the laboratory, Phase III, was to address the role of the laboratory to grow and provide future possibilities for student designed experimentation. [17] [25] A laboratory-scale model detailed by Mohagheghi, located at Georgia Institute of Technology, [18] was heavily referenced for the design and specification of the backbone of a modular, three bus laboratory-scale power system which is to be constructed and continued in design by future undergraduate students. This basic system consists of two generation sources, six loads, six single phase transformers, three transmission lines, and a connection to the utility. [18] Each generation source is associated with a separate bus. Transmission lines are configurable in length by multiples of two mile sections between each of the modular buses for design flexibility and were modeled after the Georgia Tech 
laboratory design for transmission lines. [18] Clearly labeled custom panels for the system interconnection nodes physically identify the configuration of power system components between the three buses. These buses are represented by 72 inch relay racks on castors. A load shifter, also modeled after the Georgia Tech laboratory, was designed to emulate the effect of load shifting experienced by the load bus. Each bus has been designed to hold a maximum of two loads. The load designs are left for future student projects in the laboratory.

\subsection{Laboratory Requirements}

To meet the laboratory outcomes, the laboratory was first considered from a macroscopic viewpoint, defining how the ten overarching laboratory outcomes adopted from Feisel [12] directly apply to the laboratory. The following ten outcomes are detailed with respect to their direct application in the laboratory, [13]

\section{Instrumentation (1)}

Students investigate the characteristics and limitations of current transformers, and they make measurements of parameter of various electromechanical relays.

\section{Models (2)}

Students compare physical measurements with the corresponding theoretical models, and evaluate the validity of theory learned in lecture. Students build software models to test protection coordination theory and compare results to what is expected during an actual event, determining the ability of the theory to predict real world behavior.

\section{Experiment (3)}


Weekly assignment descriptions clearly articulate test procedures, experiments and equipment. Students implement test procedures and experiments after a demonstration by the laboratory instructor.

\section{Data Analysis (4)}

Students collect, analyze and interpret data collected from testing equipment and software programs. They present these data through the written reports and through discussions with the lab instructors.

\section{Design (5)}

Students simulate power systems and coordination schemes using software tools according to specifications outlined in assignment instructions. They then test and debug simulation processes to determine design effectiveness.

\section{Learn from Failure (6)}

The laboratory assignments present many opportunities that test the students' ability to identify the reasons for unsuccessful outcomes, especially with regard to the labs covering the outmoded electromechanical industry equipment.

\section{Psychomotor (8)}

The laboratory assignments require students to properly select, analyze and operate laboratory equipment and assemble testing systems. We expect students to reference equipment data sheets and users manuals as means for teaching themselves how to operate test equipment and prepare elements for testing.

Safety (9) 
Students must complete a quiz regarding electrical safety prior to using any energized lab equipment. Students apply lock-out, tag-out procedures when using energized sources. Each lab session reviews the safety procedures during the assignment introduction and overview prior to beginning experimentation.

\section{Communication (10)}

Strong communication skills are necessary for quality engineering in any discipline. The curriculum focuses on communication between team members and lab instructors. Each lab requires students to write a technical report covering experiment theory and results. Teamwork (11)

Students work in teams of three while performing tasks in lab and synthesizing results for the written reports.

The individual laboratory assignment objectives were considered from a microscopic perspective and were individually based on the assignment experiments with the equipment and analysis of data. These individual objectives were related back to one or more of the ten laboratory outcomes of instrumentation, models, experiment, data analysis, design, learning from failure, psychomotor, safety, communication, and teamwork.

The rubric assessment of written reports assessed student knowledge of engineering and mathematical concepts, the ability of the student to synthesize and communicate understanding of these concepts and apply them to the experiment analysis, and the 
ability of the students to work in teams. In this way the ABET Student Outcomes from EC2000 were assessed and able to be evaluated, an important aspect of the goals of EC2000. 


\section{Literature Review}

\subsection{Industry Demand}

Industry demand for incoming power engineers, prompted by a previous lack in power engineering enrollments in college noted at the turn of the century, has been acknowledged as problematic for years. [4] [5] [6] The strong regional and national demand for power engineering graduates is supported by a 2011 survey from the Center for Energy Workforce Development (CEWD) projecting a 38\% turnover in industry engineers to occur between 2010 and 2015, with an additional 15\% turnover in the subsequent five years, aggregating to a need for nearly 15,000 replacements by 2020. [7] [8] Three of the large employers of power engineers in the metropolitan region, Portland General Electric (PGE), PacifiCorp and the Bonneville Power Administration (BPA), project significant levels of retirements in the immediate future, 2015-2020 (Table 1). [7] [8]

Table 1: Current Workforce \& Eligible for Retirement - 2015 \& Projected to Retire - 2015 \& Eligible for Retirement - 2020 \& Projected to Retire - 2020 [13]

\begin{tabular}{|l|l|l|l|l|l|}
\hline & $\begin{array}{l}\text { Current } \\
\text { Workforce }\end{array}$ & $\begin{array}{l}\text { Eligible for } \\
\text { retirement - } \\
\mathbf{2 0 1 5}\end{array}$ & $\begin{array}{l}\text { Projected to } \\
\text { Retire - 2015 }\end{array}$ & $\begin{array}{l}\text { Eligible for } \\
\text { retirement - } \\
\mathbf{2 0 2 0}\end{array}$ & $\begin{array}{l}\text { Projected to } \\
\text { Retire - 2020 }\end{array}$ \\
\hline PGE & 95 & 44 & $68 \%$ & 42 & $62 \%$ \\
\hline PacifiCorp & 200 & 27 & $80 \%$ & 38 & $90 \%$ \\
\hline BPA & 450 & 144 & $66 \%$ & 216 & $84 \%$ \\
\hline
\end{tabular}

To consider the implications of the lack of engineers entering the workforce, the effect the workforce has on education must be examined. Industry partnerships in engineering programs are essential to producing a professional workforce. [2] [15] [16] 
Montoya, et al, cite the restructuring, segmentation, and specialization of the power industry as a significant factor in the future of engineering education, and one that will spur innovation. [15] These realities of the power industry are reflected in Grover's research on the decline of "innovative" engineering professionals entering the workforce, for which he considers industry involvement in engineering education essential. [3] The involvement of industry in education spurs innovations in technology and educational experiences because they are driven directly by industry needs. [3] [26] Genheimer, et al, cite the effectiveness of industry partnerships at the university level for reinforcing ABET EC2000 criteria through the assessment process involved in industry-related student research projects. [30] With direct industry involvement shaping research projects and providing working industry experience for students, a regionally-focused program is developed based on the needs of the area.

Each power education program that is unique to its geographical region and, shaped by the local industry, has the potential to create more innovation and workforce-ready graduates than one of a nationally standardized form. [13] [27] The Clemson University Research Center Association cites the success of university research projects tied to positive working relationships between the university and industry partners as more evidence of the importance of active industry involvement in engineering education [2] since students are actively learning industry engineering requirements through the practice of engineering. [31] 


\subsection{The Laboratory Contribution to Engineering Education}

From the mid nineteen-eighties through the nineteen-nineties there was a decrease in engineering enrollments across the nation [19] which created a focus on both the cause of the decline and how to improve the engineering education experience to increase, and then maintain, recruitment in engineering programs. [21] The place of the laboratory in the education has become an important part of this because it provides a way "to get students involved in learning and to distribute some of the responsibility and joy of learning to them." [32] These benefits of the laboratory, combined with required SLO set out by EC2000, have the potential to integrate many of these criteria in one course. [33] [34]

Felder describes inductive learning as the process of gathering information, processing it, and then applying it to theory while deductive learning is the generality of theory that then evolves into specific applications. [35] While the educational style of lecture courses have the history of being deductive, [19] [20] the laboratory experience presents a space to facilitate cooperative based learning inherent to inductive methods of knowledge transfer. This is done by creating small, cooperative learning communities within groups, which have an increased effect on student engagement in the learning process. [34] This effect contributes positively to both retention of information and student satisfaction of the learning experience. [20] Part of the effectiveness in the education process of a laboratory is the ability of the laboratory to address the individuality of laboratory participants to introduce their own insights and experiences to increase the learning experience of the whole, [36] including the laboratory instructor, 
through the cooperative learning. The laboratory instructors are considered "as organizers and facilitators of learning opportunities", dispersing and absorbing knowledge in a reciprocal manner with students. [23]

Gleason addresses this issue of program enrollment retention with hands-on experience in the laboratory [21] citing an increase in student involvement and subsequent satisfaction in the education process. Similarly, Huet found that student engagement in the course material was important to academic success. [37] Mountain cites the importance of report writing and the improvement in written communication skills akin to those of the actual hands-on experience itself along with its importance in ABET EC2000 standards for engineering curriculum. [38] Johri's research on the effect that dialog has in learning since higher learning is acquired in the experience of the individual and the social world as the experience is synthesized. [22] Language is the means of "shared meaning making" [22] and is therefore at heart of knowledge transfer. The increase in verbal communication is another benefit of the laboratory since this verbal component also directly infers a personal involvement in the topic at hand. For these reasons, the PSRC has deemed the laboratory component of the power system protection subject is referred to as "essential". [24] To continue laboratory relevance, consistent assessment and evaluation must be made to curriculum and its delivery. Student feedback is an important aspect of this process. It gives the students a participative role in the learning process and it allows for assessment and evaluation of the curriculum at hand. [37] 


\subsection{Curriculum Review}

In 2000, Sidhu at the University of Saskatchewan identified the basic curriculum for the university power system protection course taught over a thirteen week term. The course covered principles of protection, the operation of electromagnetic (EM) and solid state relays, relaying principles, and the basic concepts for radial distribution protection and looped system protection. [39] Nine years later a similar outline for power system protection curriculum was suggested by the PSRC, reiterated these same points by stating that the power system protection education curriculum must illustrate different relay types, curve characteristics, and testing equipment. Operational selectivity of relays is also a requirement, as this relates to the coordination principles inherent to effective power system protection practices. [24]

There is a current movement to set a national power engineering curriculum covering different power system topics, with the goal of standardizing the laboratory space and equipment. [27] [28] While this standardized curriculum approach has its merits in the foundations of attempting to provide functional structure for power engineering education, it disregards the importance of the university responsibility for developing curriculum best suited to the individual resources of the educational institute as well as the surrounding geographical power industry. [3] For some laboratory courses, the traditional method of teaching the laboratory in close proximity to lecture material is still a valid approach. [25] 
A power system software curriculum component is also a requirement of the PSRC as part of preparing students for industry employment. [24] Simulation programs are used in industry to test protection coordination, determining the efficacy of the design before physical implementation. [40] Design software and control software are also used to create systems and test scaled models in a laboratory setting which mimic real power system operations. [18] [40] [41]

The assessment and revision of curriculum to continually provide effective and topical power education is an important part of meeting the EC2000 outcome criteria. In a study on the effectiveness of EC2000, Latucca et al. found that 2004 graduates were more actively engaged in their own learning and programs emphasized a greater "openness to new ideas and people." [42] This influence of openness resulting from the ABET EC2000 criteria, which supports an inductive learning style, is directly applicable to the changes involved in power engineering with regard to the new dynamics of the changing electrical grid landscape. [43] [44]

\subsection{Equipment Review}

Generically relays are used across multiple industry disciplines because they are a means of executing control over an electric system. The term "relay" in the context of power systems holds a different connotation. A generic functional description of a "relay" as defined by the IEEE states "A relay is an electric device designed to respond to input conditions in a prescribed manner and, after specified conditions are met, to cause contact operation or similar abrupt change in associated electric control circuits." [29] [45] In the 
context of power engineering, a "relay," or more specifically a "protective relay," is "a relay whose function is to detect defective lines or apparatus or other power system conditions of an abnormal or dangerous nature and to initiate appropriate control circuit action." The latter definition, from IEEE 100-1992, is the one applicable to the topics in this document. [45]

Due to the intricate nature of the subject matter of power system protection, it is necessary for the laboratory equipment to reflect both the principles of the course lecture as well as that which is found in industry. [17] [14] Both $\mathrm{EM}^{4}$ and $\mathrm{MP}^{5}$ relays are consistently used in power system protection course theory due to overcurrent protection being the fundamental principle of protection for transmission and distribution, which expands into directional overcurrent, and distance relaying. [24] [39] [46] Electromechanical relay protection is the foundation of the protection industry and holds important teaching value [13] since their mechanical construction allows for student observation of operations that are not visible in the digital relay models. [29] [47]

MP-based relay equipment have become the contemporary standard in power system protection, resulting from their ability to house many protection functions in one unit. [18] [40] [48] Today, there is an educational focus on MP-based relays in response to the technological changes seen in the power industry. [15] The focus on digital equipment is

${ }^{4}$ Electromechanical

${ }^{5}$ Microprocessor 
derived from an industry movement from EM- to MP-based systems with the advent of new types of variable generation being seen at both the utility scale down to residential level. [6] [44] As a result of this industry change, contemporary university laboratories have followed suit and have placed a focus MP relays as is the case for the University of Saskatchewan, [49] the Georgia Institute of Technology, [18] and University of Texas at Arlington. [50]

There is a focus on physical power system simulation in the power system curriculum. Mehta details a model scale power system used to simulate numerical distance protections where lumped models of transmission lines were built to accompany the 418 volt, three-phase supply which is used to test an industry standard distance relay. [46] [51] This set up is similar, but simpler, than the laboratory-scale power system model at the Georgia Institute of Technology. [18] [41] This facility currently has a modular power system protection laboratory that is used to instruct students on coordination of radial system protection and also is used for research of phasor measurement units (PMUs). This laboratory is of a modular design that includes scaled model transmission line modules designed for a $2.8 \mathrm{kVA}, 194$ volt three phase power system using a National Instruments waveform generator and a home theater amplifier capable of producing 2.8 kilowatt as a generations source combined with a set of isolation transformers to boost the line voltage from 56 volts to 115 volts. [18] [41] This same laboratory also uses a novel transmission line module assembly, which takes into account both the mutual impedance of lines and the shunt capacitance in two-mile sections made up of three 
modules. [18] In a larger scale design, the University of Texas at Arlington also utilizes a model scale power system using MP based relays inside a looped system with multiple generation sources and modeled transmission lines. [50] This laboratory is used to test relay protection principles and design. The Anhui University of Technology in Ma'anshan China has a four-bus, model-scale microgrid laboratory that models a variety of generation and employs the testing of protection theory. [52]

\subsection{Summary}

For the power systems laboratory to have an effective place in university programs, the curriculum and equipment must have relevancy to the principles and theory of the subject. This includes a focus on more than contemporary, computer based equipment. The pedagogical advantage to using historically-accurate equipment allows students to physically study the principles the power system protection industry was built upon. This outmoded equipment needs to be used in conjunction with the modern, MP-based relays to illustrate the parallels in operation and to acquaint students with current industrystandard equipment. In conjunction with this practical experience associated with equipment use and power system protection, a working power system model allows for expansion and exploration in the laboratory. Such a model provides a small-scale representation of a working power system and gives the opportunity to test, observe, and experiment with protection equipment and theory as it would be applied in industry. 
By establishing clear course outcome and assignment objectives, the PSU power systems protection laboratory has a vital effect in supporting modern power engineering education practices. Employing assessment and evaluation ensures that the curriculum stays relevant to the subject as it applied to the changing topology of the power industry and enhances student engagement and investment in the learning process. Industry partnership in the university also contributes to this feedback loop of reinforcing relevant curriculum and as it applies to the changing industry. 


\section{Laboratory Curriculum Description}

To meet educational standards as described by Brahma [24] and Feisel [11] [12]the curriculum elaborates on the lecture curriculum subjects outline as provided by the lecturing professor. The curriculum covers thermal principles of conduit, the use of industry standard software and relay equipment as well as the topics of radial and looped system protection coordination using overcurrent and directional overcurrent (DO) elements. For the laboratory, industry-standard power protection relay equipment and instrumentation transformer are tested and characterized. For all experiments, a short report detailing the assignment results was required from each group. Rubrics associated with these assignments are located in Appendix E. The order of lab assignments is intended to follow the lecture material weekly to follow theory directly with practical application.

\subsection{Lab 1: Introduction to ASPEN software}

Weekly laboratory assignments begin with an introduction to ASPEN, an industry standard software package used to design protection coordination schemes. This first lab introduces students to the software used in subsequent labs while concurrently providing a week of course lecture material to be taught. For this first assignment, students build a model of a simple radial system (Figure 1) and a simple looped system (Figure 2). 


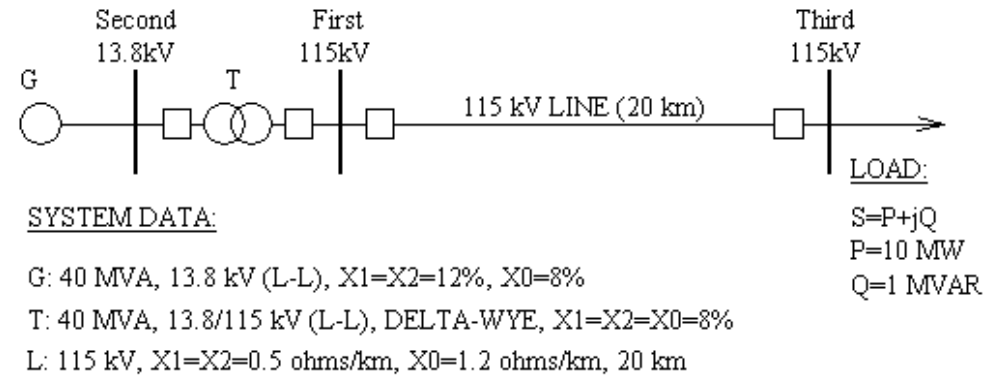

Figure 1: An example of a radial system model in the ASPEN modeling software package. Source: L. Perez.

Students start creating the model by choosing a system MVA base, and then follow written instructions for adding components into the system with the values provided in Figure 1. At this time relay groups are not added to the circuit breakers since the goal of this first lab being analysis of the power flow under a single bus contingency and not the design of a protection scheme. A triple-line-to-ground and a single-line-to-ground fault with no outage are simulated close in to the line side circuit breaker next to the bus marked FIRST. Students are required to include an image of one-line system diagram results from the desktop and save the TTY report results generated by ASPEN for the assignment report and to determine the neutral currents of the transformers under these faults. The simulated results are then compared to calculated values for the system for the fault currents. The models are saved for later assignments involving relay coordination with ASPEN.

The same procedure is repeated for the looped system in Figure 2. Close-in faults for triple-line-to-ground and single-line-to-ground are performed at all buses. A new line is 
added between Stations $\mathrm{R}$ and $\mathrm{K}$ with impedance parameters of $\mathrm{L}=100 \mathrm{mi}, \mathrm{X} 1=0.5 \mathrm{pu}$, $\mathrm{X} 0=1.5 \mathrm{pu}$ and the same fault studies are repeated with the addition of the new line and a line-to-line fault are simulated at the midpoint of the new line to demonstrate the difference in system response with a new line and fault location. The sequence components $0-1-2$ and the phase a-b-c currents and voltages at each end of the new line are recorded with the change in power flow results after the addition of the new line.

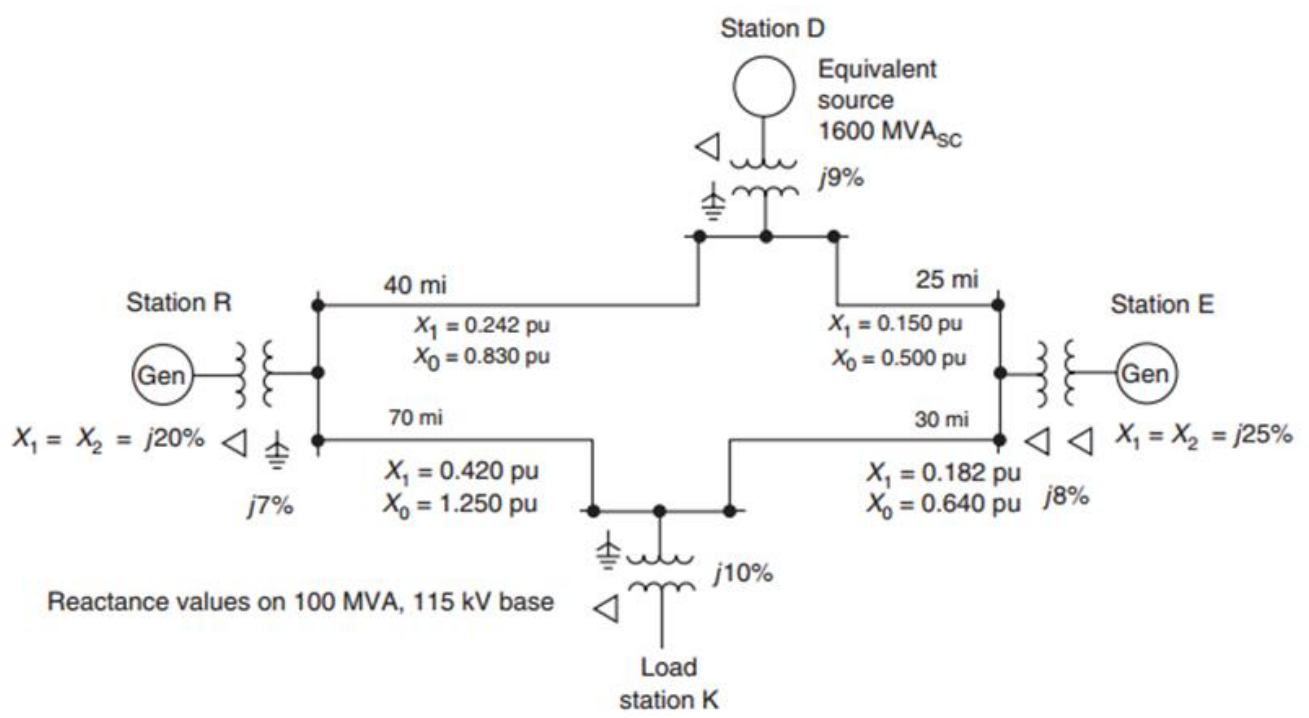

Figure 2: An example of a looped system model from the Blackburn text [29]

\subsection{Lab 2: Thermal properties of a conductor in free air}

The second laboratory assignment covers the thermal properties associated with bare copper wire. The assignment requires applying a current source to different copper wire gauges of 8 AWG and 10 AWG. The temperature vs. time relationship is explored experimentally for the conductor's temperature response to a constant amount of current over time. Similarly, the current vs. time relationship is explored experimentally by testing the melting points of a 24 AWG copper wire to representing fuse damage 
behavior. The inverse relationship of conductor damage to time is the basis for all power system protection theory because it indicates the amount of time to equipment failure under elevated thermal conditions experienced with increased current flow. To illustrate these relationships examples of the experimentally derived temperature vs. time curves compared to theoretical calculations for the copper material and wire gauge at two different levels of current (Figure 5). The temperature scale is given in degrees above ambient temperature of $72^{\circ} \mathrm{F}$.

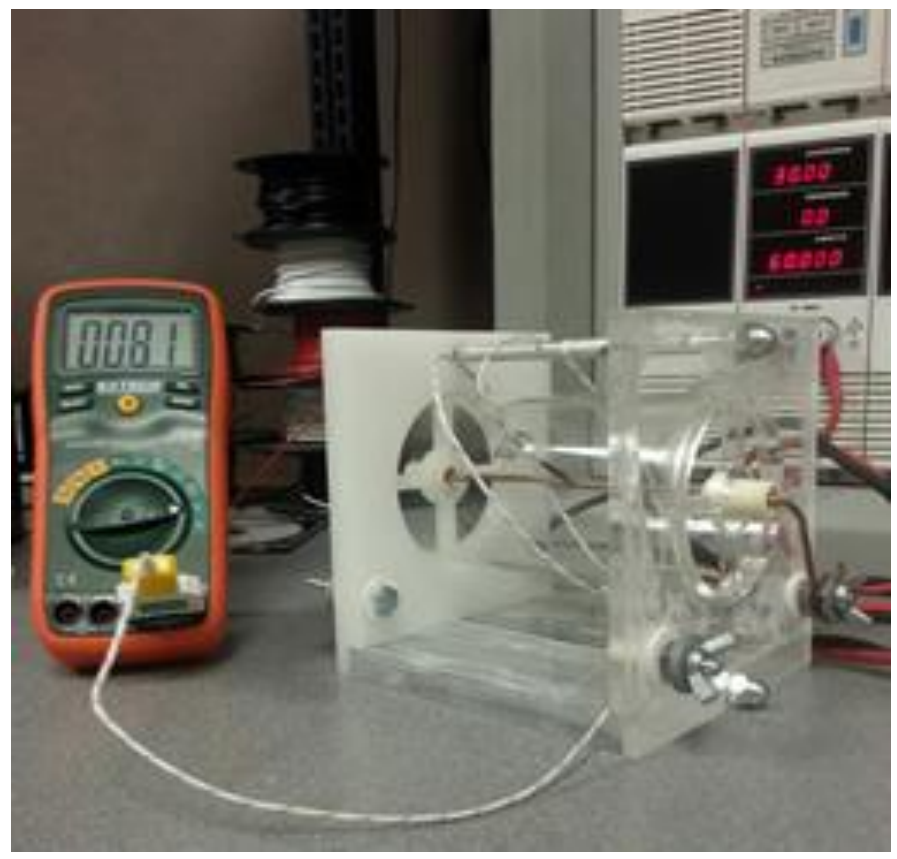

Figure 3: Thermal properties experiment: physical implementation of wire being tested with a high current source connection and thermocouple measuring temperature. Acrylic fuse holder was designed specifically for this application. Source: Ferris 


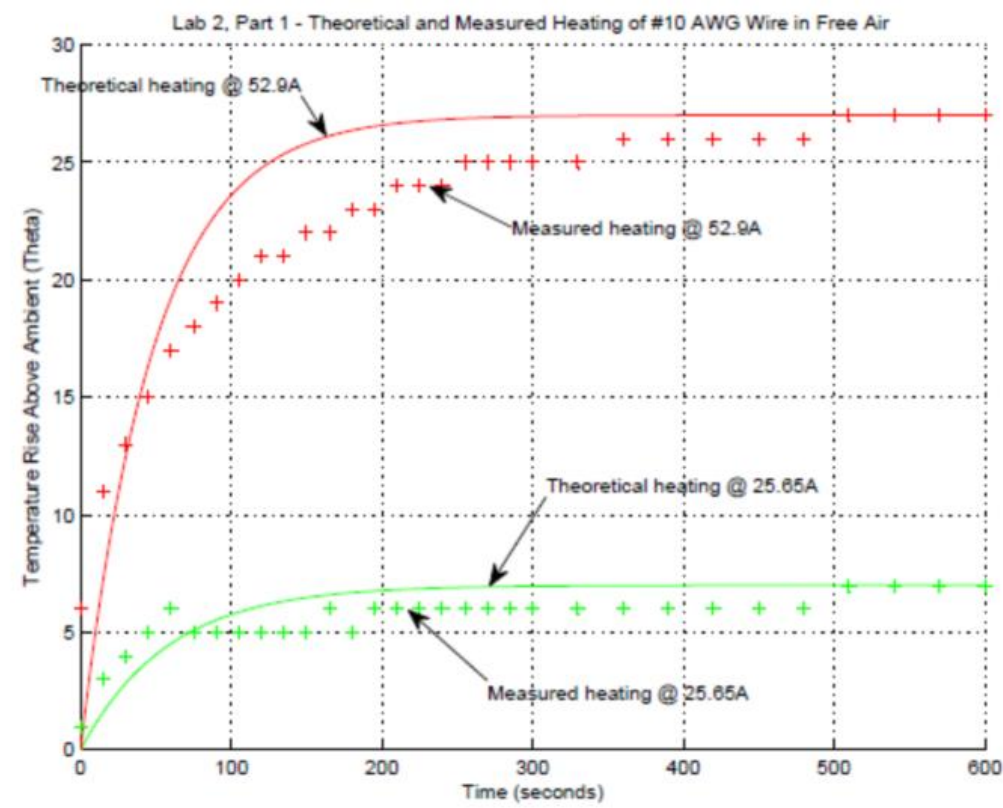

Figure 4: Comparison of experimental to theoretical T(t) curve. Expand more. Describe the purpose of this plot, with respect to the curriculum.

Source: Student results

Using a 24 AWG size wire, the minimum melting time and total clearing time of a fuse element is found using the results of experiments by heating the copper conductor at several different current levels and recording the response of the wire visually. The experiment is set up with the fuse holder as shown in Figure 1.

The minimum melting time, $\mathrm{t}_{\mathrm{mm}}$, is difficult to measure physically, so the time the wire changes colors to glowing orange is used to represent this time. The fuse total clearing time, $t_{c c}$, is measured at the time the copper conductor broke. Figure 5 shows an example of student results for this experiment. 


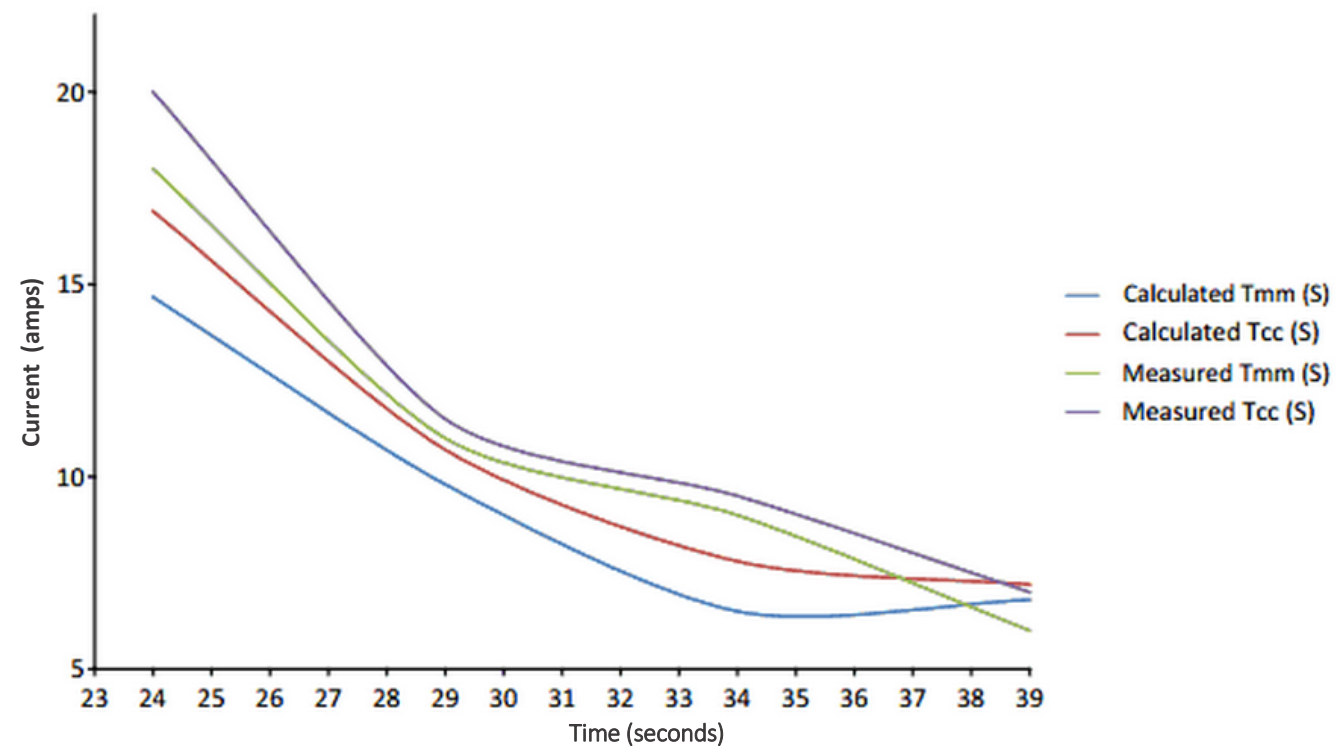

Figure 5: Fuse damage curve, I(t)

Source: Student results

The equations used to define the melting time curve is given in lecture theory as,

$$
I * t_{m m}=\frac{C D A^{2}}{\rho_{0} \alpha_{0} \ln \left(1+\alpha_{0}\left(T_{m}-T_{0}\right) / 1+\alpha_{0}\left(T_{i}-T_{0}\right)\right)}
$$

Similarly the total clearing time equation is given as,

$$
I * t_{c c}=\frac{C D A^{2}}{\rho_{0} \alpha_{0} \ln \left(1+\alpha_{0}\left(T_{d}-T_{0}\right) / 1+\alpha_{0}\left(T_{i}-T_{0}\right)\right)}
$$

Using constants provided in lecture theory,

- $\mathrm{C}$ is the specific heat capacity of copper

- $\mathrm{D}$ is the density of copper

- $\mathrm{A}$ is the area of the copper conduit

- $\alpha_{0}$ is the temperature coefficient of copper at the nominal temperature of 20 degrees Celsius, given in units of $1 / \mathrm{K}$

- $\rho_{0}$ is the resistivity of copper at the nominal temperature of degrees Celsius, given 
in units of $\Omega * m$.

- $\mathrm{T}_{0}$ is the nominal temperature of 20 degrees Celsius

- $\mathrm{T}_{\mathrm{a}}$ is the ambient temperature of the surrounding free air

- $\mathrm{T}_{\mathrm{i}}$ is the initial temperature of the surrounding free air

- $\mathrm{T}_{\mathrm{m}}$ is the minimum melting temperature

- $\mathrm{T}_{\mathrm{d}}$ is the damage temperature at the point of the total clearing time, $\mathrm{T}_{\mathrm{cc}}$

Again, it is important to compare the measured values to the calculated values from the course lecture theory to illustrate the similarities and differences of the theoretical and practical applications of this subject. A report of the assignment results is required from each group.

\subsection{Lab 3: Auxiliary Electromechanical (EM) Relays}

To introduce the basic concepts of electromechanical relay operation and testing, and to give time for students to learn and synthesize the new concepts of relay setting and operations, the current and voltage EM auxiliary relays are introduced in the third lab assignment. At the start of this laboratory session all groups gather together with the assigned relays while the laboratory instructor details all working parts of the relays, including the current pick-up set screw and related contacts. The plunger contraption of the relay is not visible, but runs through the core of the instrument (Figure 6). These are tested using the MultiAmp relay equipment. Each relay type operates under the same design; the resulting force due to the flux associated with the applied current moves the plunger, which controls the position of the instantaneous OC contacts. 
The only requirements for this lab are to record the current pick-up setting for the instantaneous element contacts, compare that value to the current output listed by the MultiAmp tester, and then note the precision of the calibration of the instrument. A timing test on the instantaneous element was also performed in order to demonstrate the quick operation of the instantaneous $\mathrm{OC}$ element and to experience running an $\mathrm{OC}$ operation timing test. Overall the applied part of this lab assignment is concise and brief since the introductory equipment demonstration and tutorial performed by the TA is comprehensive and involves answering questions from the group before beginning the relay testing. The introduction makes up a significant portion of this laboratory assignment.

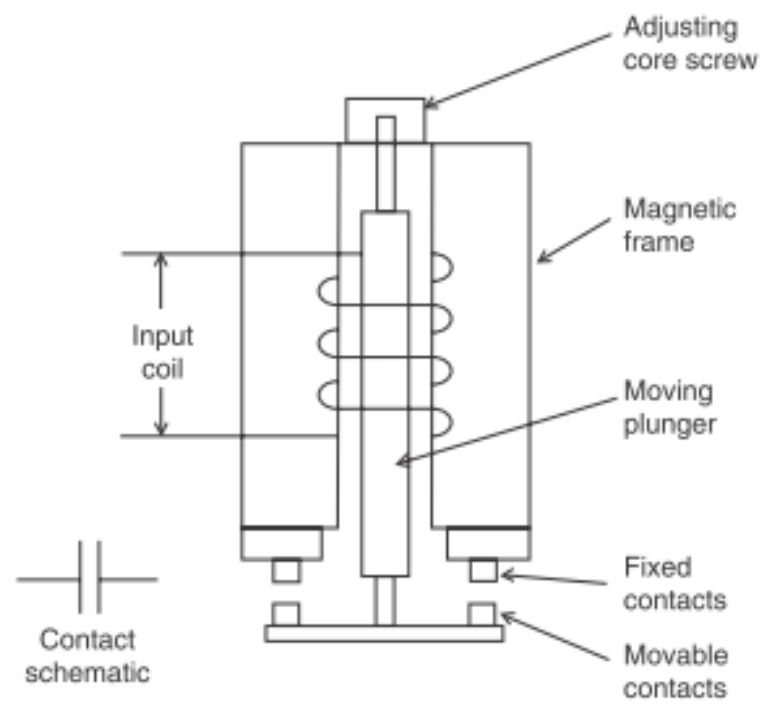

Figure 6: Generic plunger style auxiliary relay diagram [29]

\subsection{Lab 4: Current Transformer Testing}

For the fourth laboratory assignment, current transformer core saturation is tested for 
different turns ratios under overcurrent conditions and overburden conditions. The MultiAmp relay testing units are used to source the required high currents. The magnetization curve of metering-class CTs are found experimentally by taking the CT through a range of voltages on the secondary terminal until the CT is driven into saturation. Students use this experimental curve to determine the rated burden of the CT, found at the knee-point of the magnetization curve, and compare it to the manufacture's burden rating. The manufacturer provides rated burden in VA units, so students perform calculations to determine the nominal rated burden in ohms using the rated current of the CT.

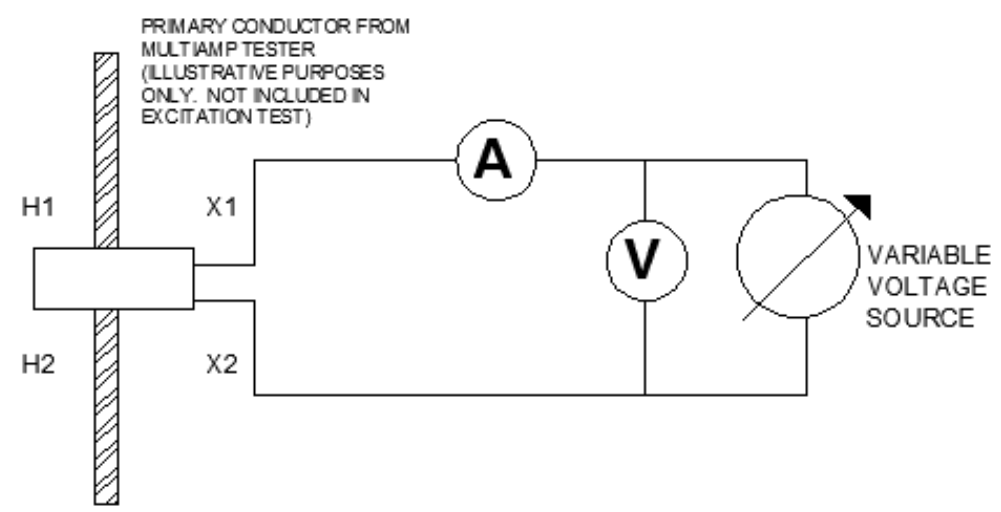

Figure 7: CT magnetization testing connection diagram.

Source: Ferris 
Table 2: CT manufacturer specification

\begin{tabular}{|c|c|c|c|}
\hline Model & Ratio & $\begin{array}{c}\text { Accuracy at } \\
\mathbf{6 0 ~ H z}\end{array}$ & $\begin{array}{c}\text { Burden } \\
\text { Capacity in } \\
\text { VA }\end{array}$ \\
\hline AL500 & $50: 5$ & $3 \%$ & 2.0 \\
\hline AL101 & $100: 5$ & $1 \%$ & 2.0 \\
\hline
\end{tabular}

The CTs for this lab are provided by a donation from Veris Industries and have turns ratios of 100:5 amps and 50:5 amps. These CTs are purchased by Veris from another supplier and, as a result, have no associated manufacturer's magnetization curve available to compare the experimental curve with. Students visually located the knee point of their experimental curve and use the corresponding voltage and current amounts to calculate the rated burden, comparing this to their calculated values of rated burden from the CT datasheet (Table 2). An example of the experimental magnetization curve from the 50:5 CT experiment produced during the course term is shown in Figure 8.

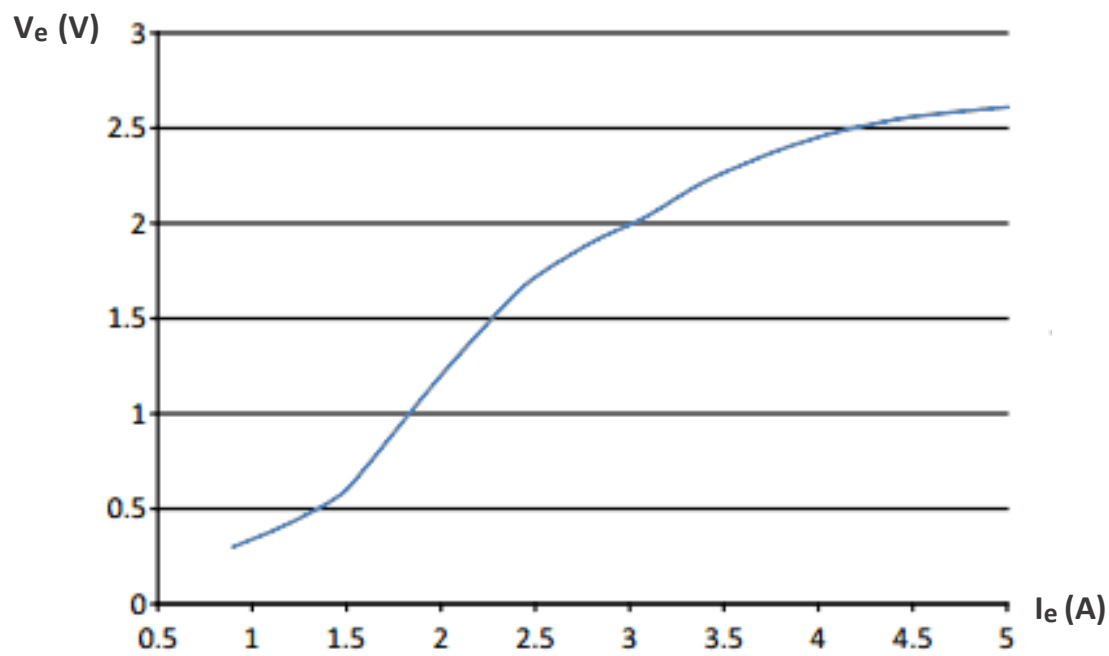

Figure 8: Magnetization curve of AL500 Veris CT with turns ratio 50:5. Source: Student results 
Overcurrent and overburden tests were performed by students in the second part of this lab assignment by using 4 AWG conductor connected to the 250 A output of the MultiAmp SR-51 series testers and ramping slowly ramping the current to at least $120 \%$ above the rated current for the 50:5 CTs. As students increase the current, they monitor the current waveforms visually for the CT response to overcurrent input on the primary at rated burden (Figure 9). The current waveforms for the results of the CT measurements are recorded and discussed as part of the deliverables for the first part of the assignment.

The overburden tests require all three of the rheostats to be connected in series. This is because the rheostats are carefully designed for the CT rated burden of 0.08 ohms at the 5 amp rated current on the secondary terminals of the CTs. The rheostat loads are not oversized to be able to provide an over-burden individually. The results of the overburden tests are recorded from the oscilloscope display as part of the deliverables for the second part of the assignment (Figure 10). 


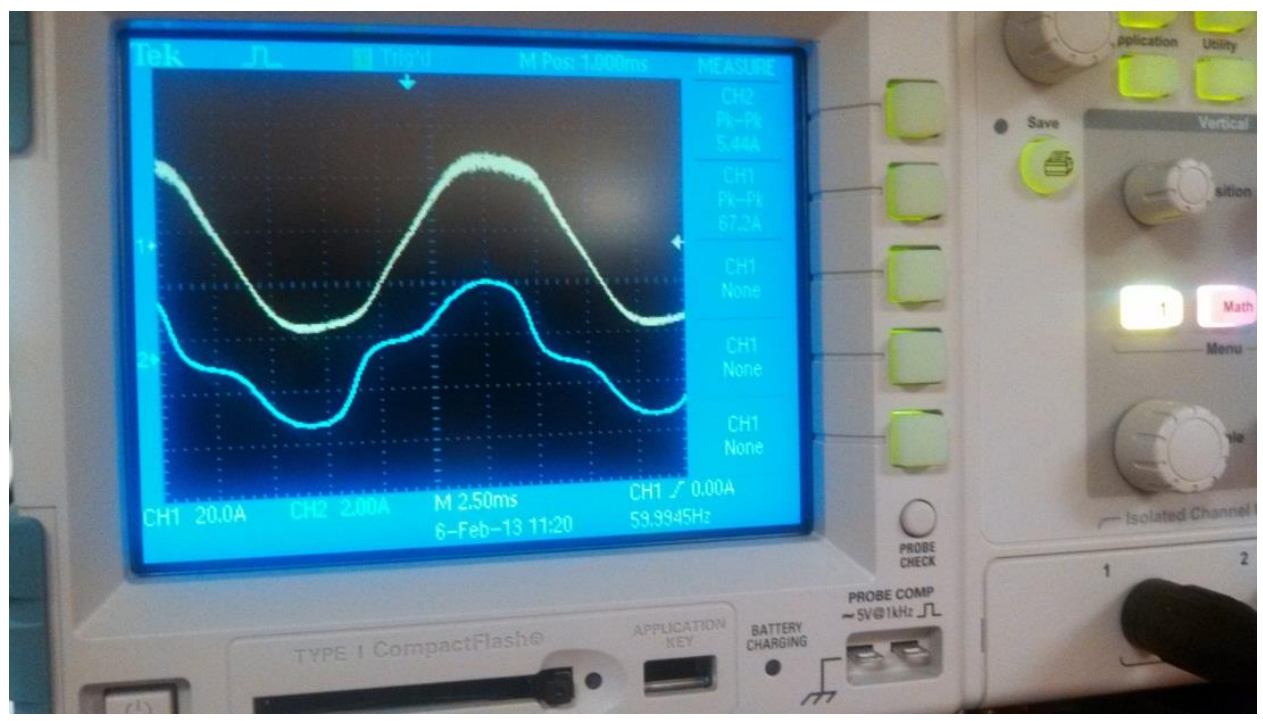

Figure 9: Measurement of a CT to $120 \%$ over nominal primary current at rated burden Source: Student results

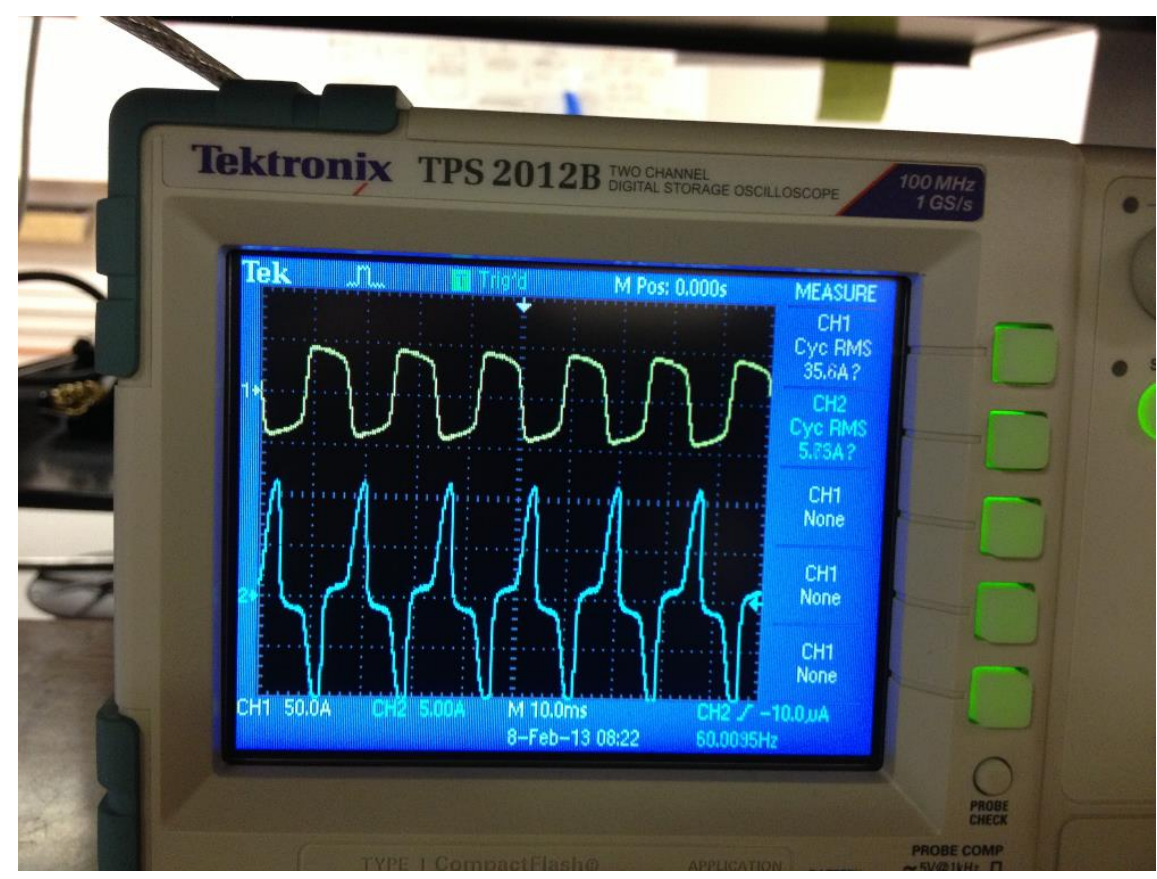

Figure 10: Measurement of a CT to $300 \%$ over rated burden at nominal primary current Source: Student results 


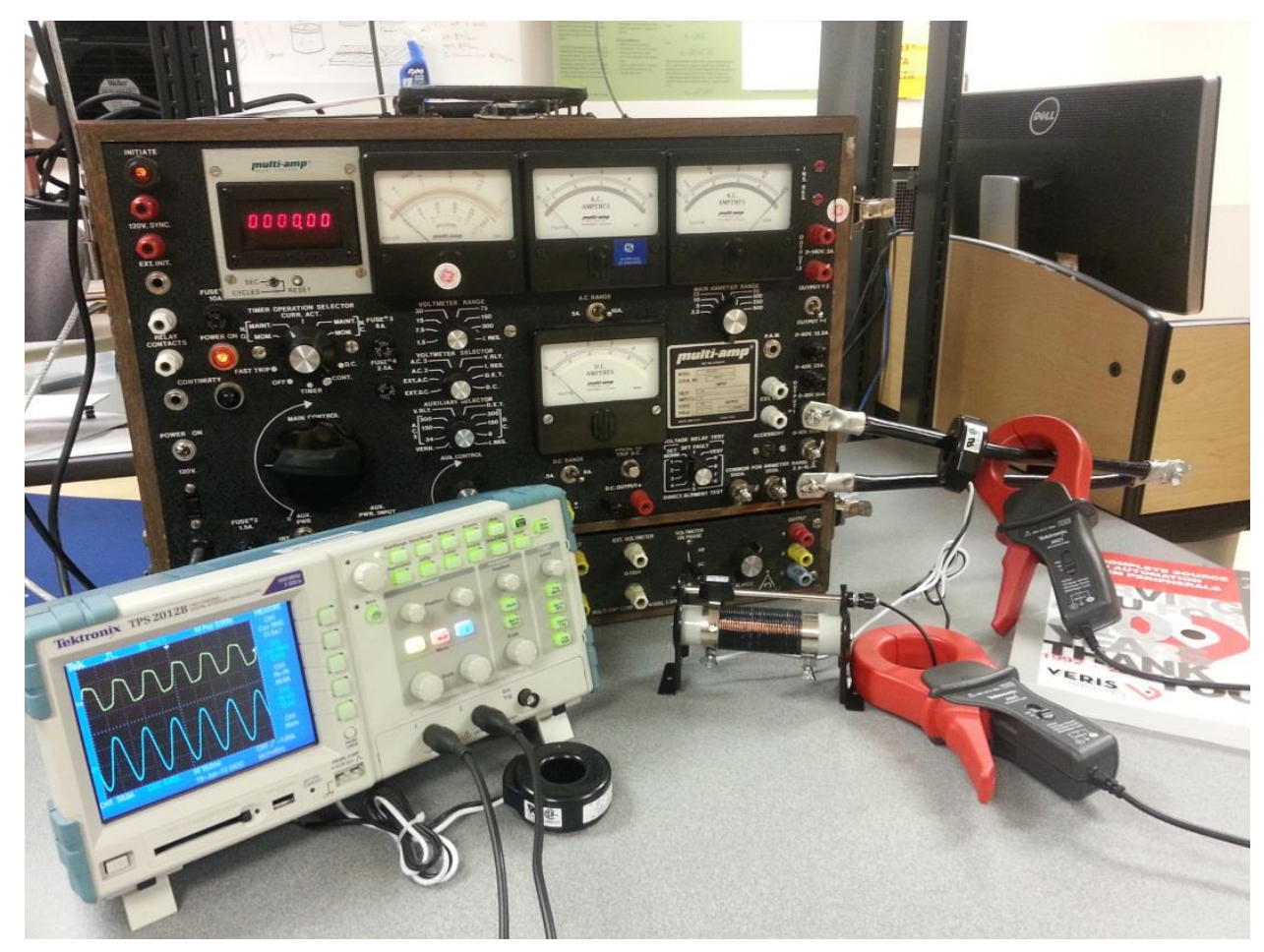

Figure 11: Physical setup for CT testing using the MultiAmp SR-51A relay testing unit Source: Ferris

\subsection{Lab 5: Electromechanical Overcurrent Relays}

The fifth laboratory assignment experiments with overcurrent EM relays donated from

Portland General Electric. The EM relays are the same equipment described and used as examples in theory from lecture coursework. The relays have a time delay element with an induction disc that operates under the force of magnetic flux generated by the current applied to a permanent magnet (Figure 12). 


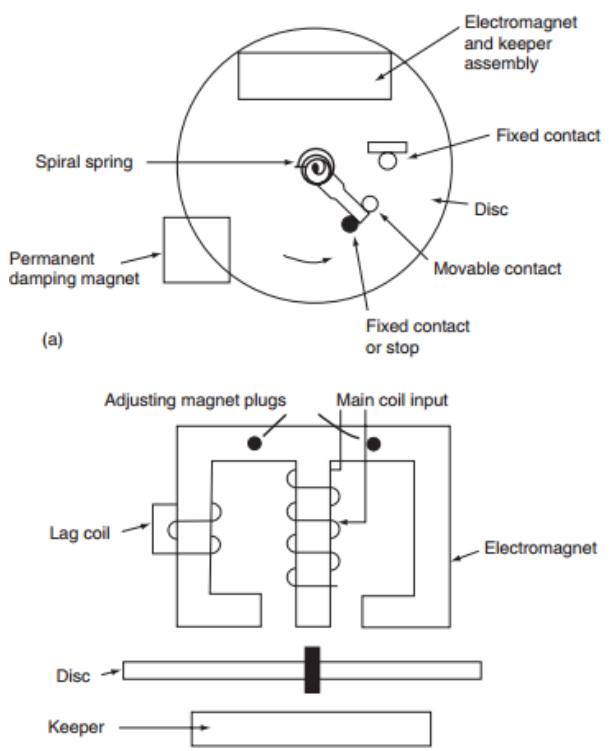

Figure 12: Diagram of induction disc relay. Source: Blackburn [29]

The relays in the labs operate on inverse and very-inverse time curves of EM relays made by General Electric,

- IAC53B - US Very Inverse Curve (U3) (Figure 13)

- IAC77B - US Extremely Inverse Curve (U4) (Figure 14) 


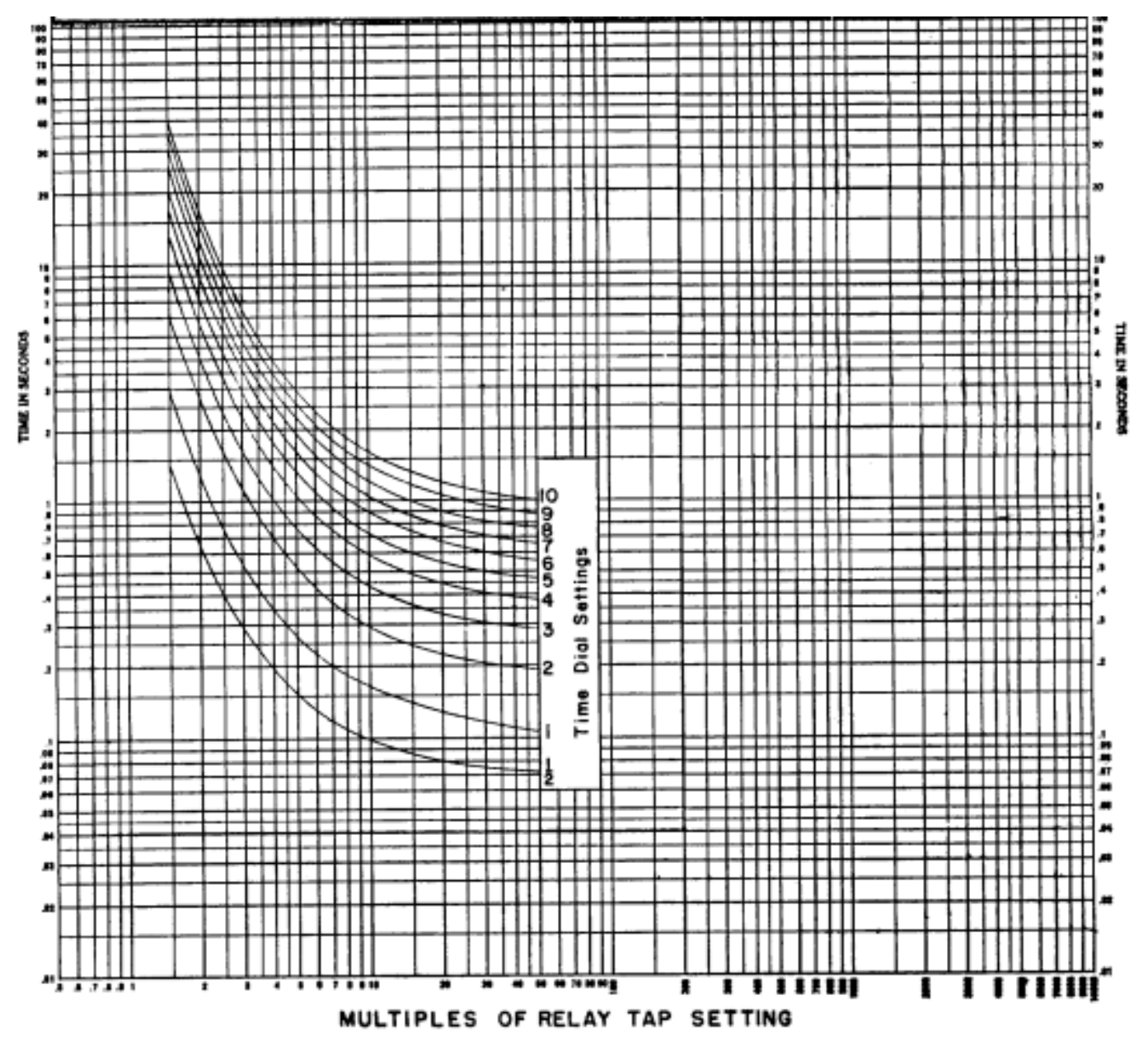

Figure 13: US Standard U3 very inverse curve from the General Electric IAC53 instruction manual. Source: General Electric ${ }^{6}$

${ }^{6}$ General Electric IAC instruction manual,

http://www.gedigitalenergy.com/products/manuals/iac/geh1788.pdf 


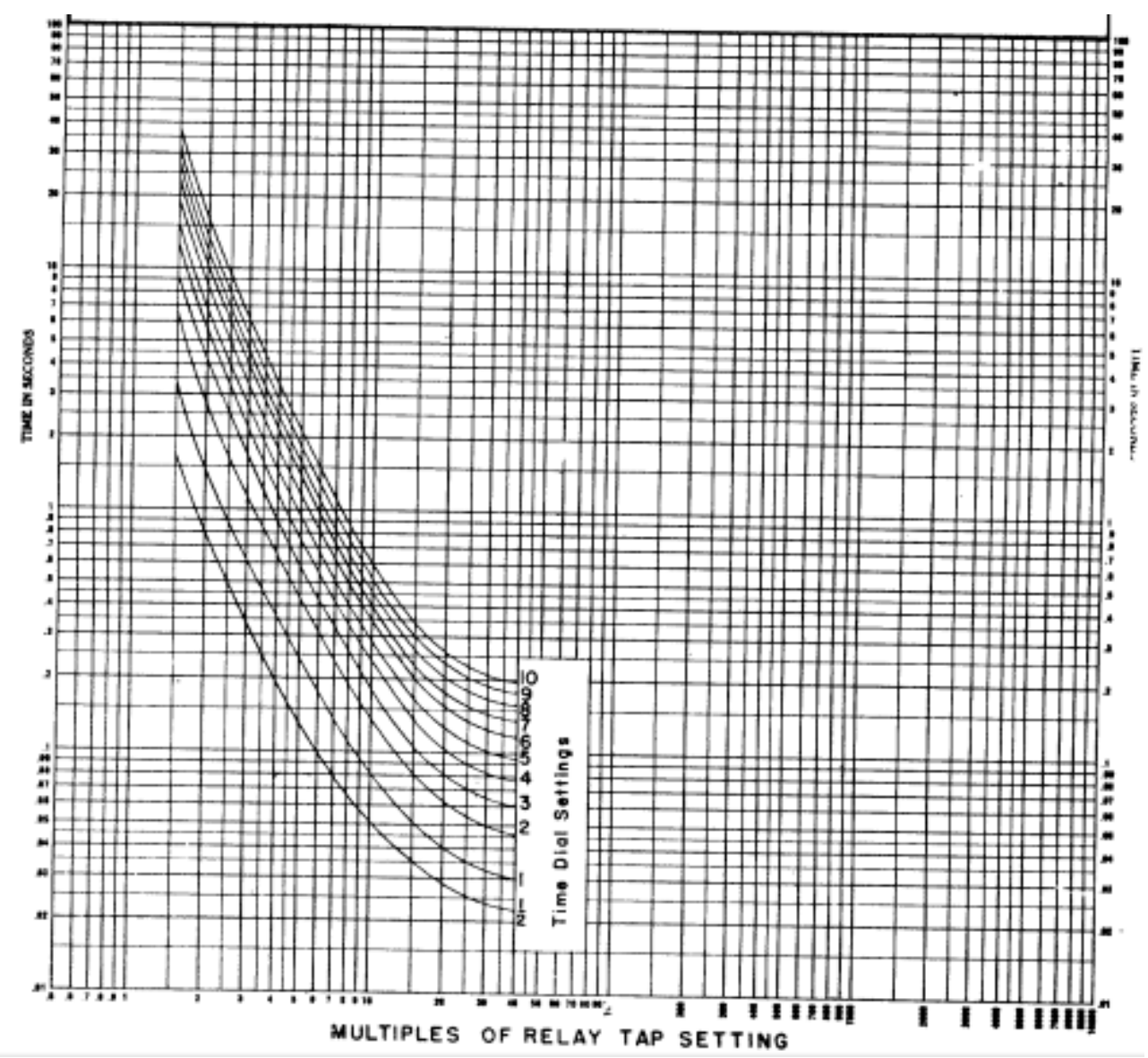

Figure 14: US Standard U4 extremely inverse curve from the General Electric IAC77 instruction manual. Source: General Electric ${ }^{7}$

The corresponding equation to calculate the operating time for the U3 very inverse U.S. curve is,

$$
t_{o p}=T D\left(0.180+5.95 / M^{2}-1\right)
$$

A similar operating time equation for the U4 extremely inverse U.S. Curve is,

$$
t_{o p}=T D\left(0.0963+3.88 / M^{2}-1\right)
$$

Where $\mathrm{M}$ is the multiple of the pick-up current and is calculated as,

${ }^{7}$ IAC77 instruction manual, General Electric,

http://www.gedigitalenergy.com/products/manuals/iac/geh2059.pdf 


$$
M=I_{\text {fault }} /\left(I_{\text {pick-up }} * C T R\right)
$$

Students test both types of curves on the MultiAmp relay testers and verify the timeinverse operating times of the two relay types by calculating the operating time based on their relay settings of TD and pick-up current, as well as the supplied test current. The students then produce a short report of their results, comparing experimental values to calculated values and make note of discrepancies between the theoretical calculations and tested conditions.

\subsection{Lab 6: ASPEN radial OC coordination}

For the ASPEN radial coordination, a modified model of the radial system from the first lab assignment is used (Figure 15). The system model is modified to add relays to the circuit breakers. To synthesize other laboratory exercises, one of the models of OC relays from fifth laboratory assignment testing EM OC relays, the IAC53 operating on US Standard U3 very inverse time curve, is selected as the time OC devices controlling the circuit breaker operations. This is done purposefully in order to be able to compare simulation results with experimental results in future lab assignments involving digital relays and to relate the operation of the model system relays to those of the EM relays tested in the previous assignment. 


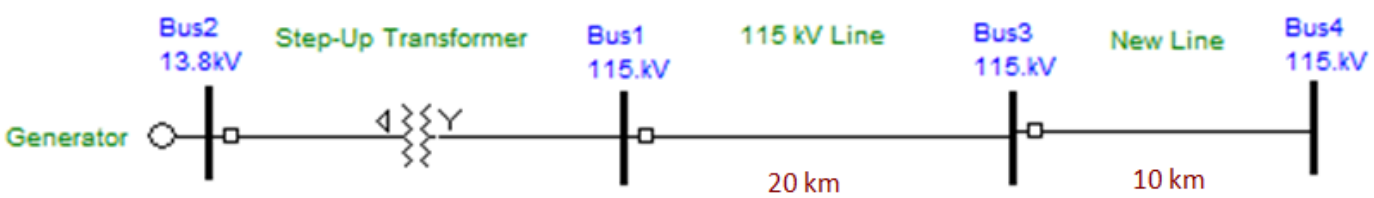

Figure 15: Laboratory 6 ASPEN radial system. Source: Ferris

Students simulate a three-line-to-ground fault at the same point on Bus 1, as was done in the Lab 1 assignment, to affirm the results are still the same as previously recorded in the first laboratory assignment. Students record fault current values along with a figure of the model. Results are verified by the TAs. After determining the accuracy of the model, students delete the load on Bus 3 and add a $10 \mathrm{~km}, 115 \mathrm{kV}$ line to a fourth bus with the following impedances,

- $\mathrm{Z}_{\mathrm{L} 1}=\mathrm{Z}_{\mathrm{L} 2}=\mathrm{j} 0.1$ p.u.

- $\mathrm{Z}_{\mathrm{L} 0}=\mathrm{j} 0.3$ p.u.

Circuit breakers for the new line are then added, with relay groups, with the following specifications. For the circuit breaker nearest Bus 3 on the $10 \mathrm{~km}$ line,

- ID: R1

- Relay Type: General Electric IAC53

- CT Ratio: $400 / 5=80$

- I $_{\text {pick-up: }} 5$ amps

- Time Dial: 3

- Instantaneous: 100,000 amps 
For the circuit breaker nearest Bus 1 on the $20 \mathrm{~km}$ line:

- ID: R2

- Relay Type: General Electric IAC53

- CT Ratio: $600 / 5=120$

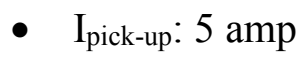

- Time Dial: 1.5

- Instantaneous: 100,000 amps

A close-in, three-phase fault to Bus 3 is simulated and the results of the relay operation and fault current are recorded with time operations on the simulated model. These results are compared to the calculated values found using the time operation equations and the provided curves. The results are documented as part of the deliverables for the assignment.

\subsection{Lab 7: Digital relay (SEL-551) testing $(50 / 51)^{8}$}

In this lab assignment, students are given an introduction the digital relays and testing equipment by setting and testing the basic SEL OC relay, SEL-551 (Figure 16). This relay has both instantaneous OC operation (50) and time OC operation (51).

${ }^{8}$ IEEE/ANSI standard device numbers for electrical power system device function numbers, acronyms, and contact designations under IEEE Standard C37.2-2008. [53] 


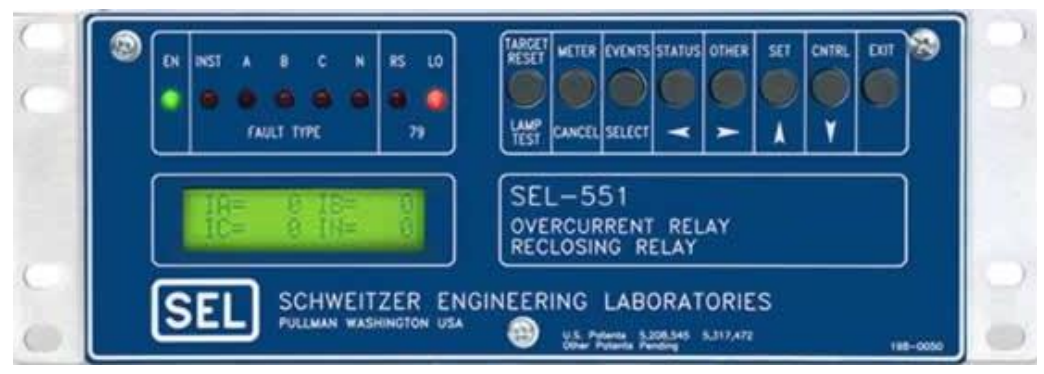

Figure 16: SEL-551 digital overcurrent relay.

Source: $S E L^{9}$

The relay is programmed with a U3 curve, and the 120 CTR of the relay group located at Bus 1 from the ASPEN radial coordination of Lab 6. The three-phase primary fault current results for relay $\mathrm{R} 1$ on Bus 1 is used in the SEL-AMS test software to test the relay operation against the same conditions found in Lab 6. Students then compare the tested operating values to the calculated and simulated results from the ASPEN radial coordination assignment from the previous week (Figure 17).

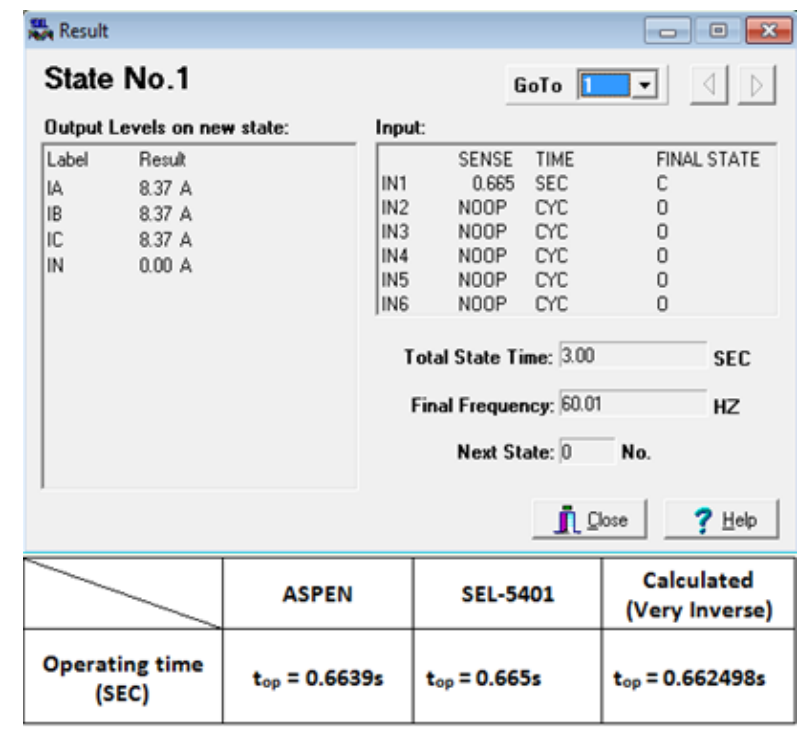

Figure 17: SEL-551 inverse time OC element testing results. Source: Student results

${ }^{9}$ SEL-551Instruction Manual, Schweitzer Engineering Laboratories, selinc.com 


\subsection{Lab 8: ASPEN looped system coordination}

The ASPEN looped system is built based on the looped system model, but was altered to reflect a more simple system with three looped buses (Figure 18). Relay tags are provided to maintain congruency in the system design for the lab groups (Table 3). All relay types are specified as the same, but students are required to decide where regular OC relays are used in the model instead of directional overcurrent relays (DOR) and deactivate the DO on the appropriately relays in the relay settings. The parameters given for the relays are,

- Relay Type: JBC51

- CT Ratio: $400 / 5=80$

- TAP: $5 \mathrm{~A}$

- Time Dial: 3

- Instantaneous: $100,000 \mathrm{~A}$

- Directional Time Element: (student discretion)

- Directional Instantaneous: unchecked 


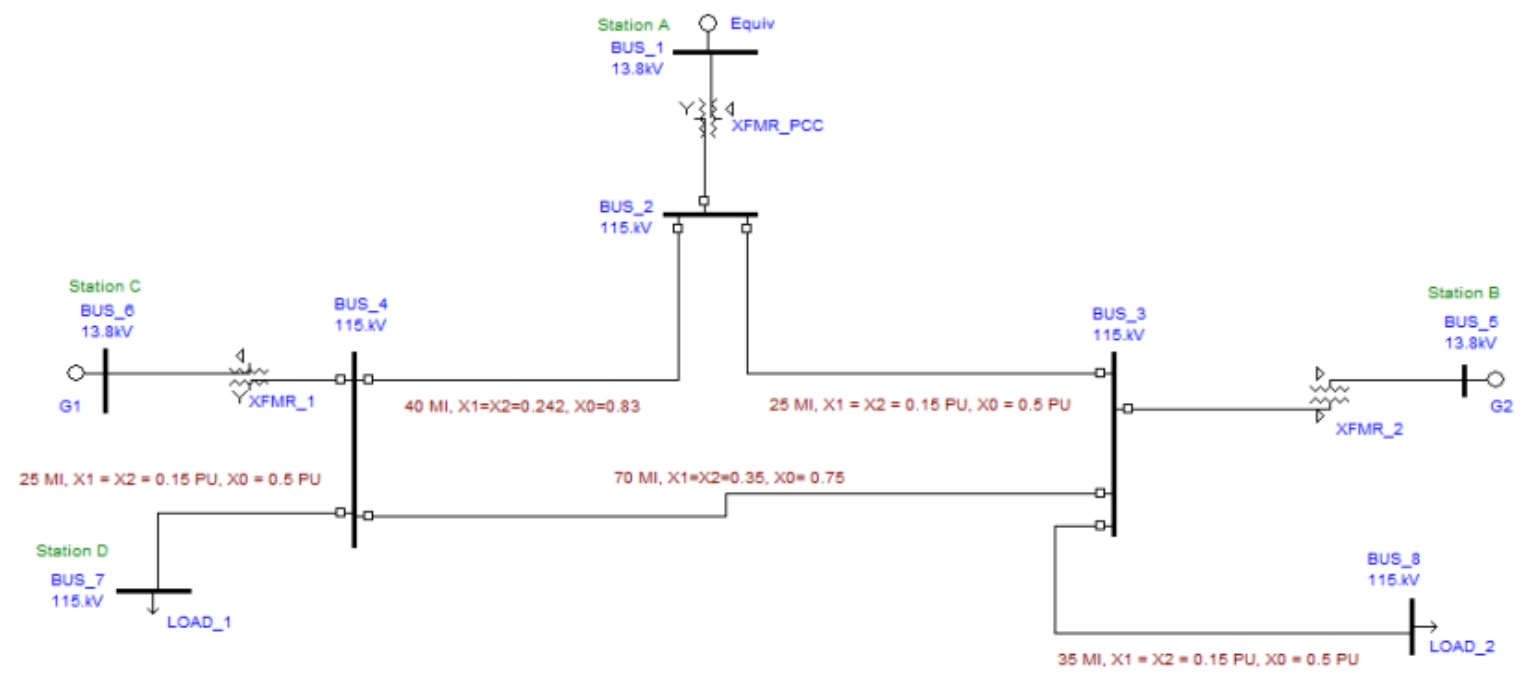

Figure 18: A three-bus looped power system modeled in ASPEN software for the ninth laboratory assignment covering looped system coordination design Source: Ferris

Table 3: Relay tags for looped system

\begin{tabular}{|l|r|r|}
\hline Relay & From Bus & To Bus \\
\hline R1 & 2 & 1 \\
R2 & 4 & 6 \\
R3 & 2 & 3 \\
R4 & 2 & 4 \\
R5 & 4 & 7 \\
R6 & 3 & 2 \\
R7 & 3 & 8 \\
R8 & 3 & 4 \\
R9 & 4 & 3 \\
R10 & 4 & 2 \\
R11 & 3 & 5 \\
\hline
\end{tabular}


And system parameters are given as,

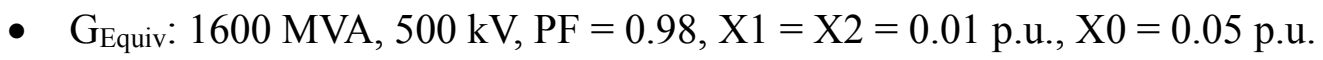

- $\mathrm{G}_{1}$ : $80 \mathrm{MVA}, 115 \mathrm{kV}, \mathrm{PF}=0.98, \mathrm{X} 1=\mathrm{X} 2=0.16$ p.u., $\mathrm{X} 0=0.1$ p.u.

- $\mathrm{G}_{2}$ : $100 \mathrm{MVA}, 115 \mathrm{kV}, \mathrm{PF}=0.98, \mathrm{X} 1=\mathrm{X} 2=0.135$ p.u., $\mathrm{X} 0=0.09$ p.u.

- $\quad \mathrm{LOAD}_{1}: 115 \mathrm{kV}, 10 \mathrm{MW}, 1 \mathrm{MVAR}$

- $\mathrm{LOAD}_{2}: 115 \mathrm{kV}, 10 \mathrm{MW}, 1 \mathrm{MVAR}$

- $\mathrm{XFMR}_{1}: 13.8 / 115 \mathrm{kV}, \mathrm{X} 1=\mathrm{X} 0=0.1$

- $\mathrm{XFMR}_{2}: 13.8 / 115 \mathrm{kV}, \mathrm{X} 1=\mathrm{X} 0=0.09$

- $\quad$ XFMR $_{P C C}: 500 / 115 \mathrm{kV}, \mathrm{X} 1=\mathrm{X} 0=0.02$

Using the provided system and relay information, combined with the example 12.8 from the Blackburn textbook on page 432 covering looped system relay coordination, [29] coordination pairs are determined by performing close-in, three-line-to-ground faults on each bus separately. These individual bus contingencies are performed first clockwise and then counterclockwise around the loop, starting at the equivalent source on Bus 2 (Figure 18). The difference in analysis direction methods simplifies the coordination process, since even in this small system there are eleven relays to be considered. Relay pairs are designed based on the simulation results of this clockwise and counterclockwise analysis around the loop. This determines the time dial settings of the relays in the loop with an ideal coordination interval range of 0.3 to 0.4 seconds for the far-bus fault and an ideal operation in less than 0.2 seconds for close-in faults. Student coordination designs are recorded in an organized presentation of the relay coordination pairs. 
Students are verbally instructed to note the language in the Blackburn 12.8 example on page 435 that gives clues to the difference between the theory and practice of protection design, "assuming that relays at breaker 8 can eventually be set to operate for close-in fault 26 at no more than 0.24 seconds." [29] In the protection design field, it is crucial for engineers to be able to understand the concept of design and how this concept breaks from the mental rigidity of idealizing theory because of the requirement for flexibility and educated personal judgment.

\subsection{Lab 9: EM and Digital DOR testing (67) ${ }^{8}$}

By the time of the last assignment of the term, students are exposed to the testing equipment and procedures from working on the other laboratory assignments, so the DO testing on the EM and digital models are combined into the same assignment. For the EM model of DOR, students are required to download the JBC51N user manual and with the TA identified the terminals associated with voltage and current source inputs as well as relay contacts used in the Timing Test and Directional Unit Test. The Pulsar user manual is also referenced for the testing procedure of the directional unit of the JBC51N relay with a US Standard U2 inverse curve (Figure 19). 


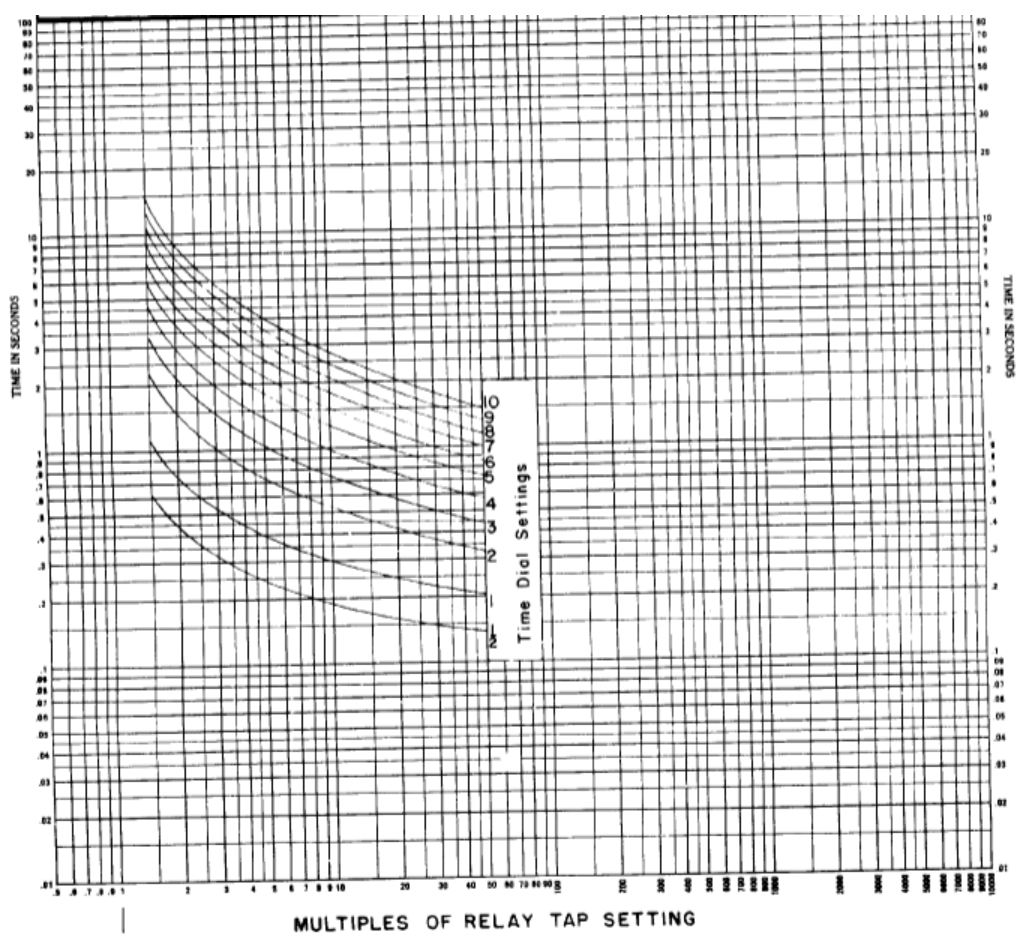

Figure 19: General Electric JBC51N US Standard U2 inverse curve. Source: General Electric ${ }^{10}$

The assignment requires students to follow the procedure outlined in the Pulsar instruction manual, using the JBC51N user manual as needed. The written lab assignment instructions give the phase angle direction over ranges from the Pulsar instruction manual and give the students directions on where to look in the manual to set the phase angle relationships (Table 4).

${ }^{10}$ JBC51 instruction manual, General Electric, http://www.gedigitalenergy.com/products/manuals/jbc/gek49848.pdf 
Table 4: Current flow direction for lagging current angles ${ }^{11}$

\begin{tabular}{|c|c|c|c|c|c|c|c|c|}
\hline $\begin{array}{c}\text { P.F. } \\
\text { Angle- } \\
\text { Lag }\end{array}$ & $\mathbf{9 0 - 1 3 5}$ & $\mathbf{1 3 5 - 1 8 0}$ & $\mathbf{1 8 0 - 2 2 5}$ & $\mathbf{2 2 5 - 2 7 0}$ & $\mathbf{2 7 0 - 3 1 5}$ & $\mathbf{3 1 5 - 3 6 0}$ & $\mathbf{0 - 4 5}$ & $\mathbf{4 5 - 9 0}$ \\
\hline $\begin{array}{c}\text { Power } \\
\begin{array}{c}\text { Flow }- \\
\text { kW } \\
\text { IN/OUT }\end{array}\end{array}$ & OUT & IN & IN & IN & IN & OUT & OUT & OUT \\
\hline
\end{tabular}

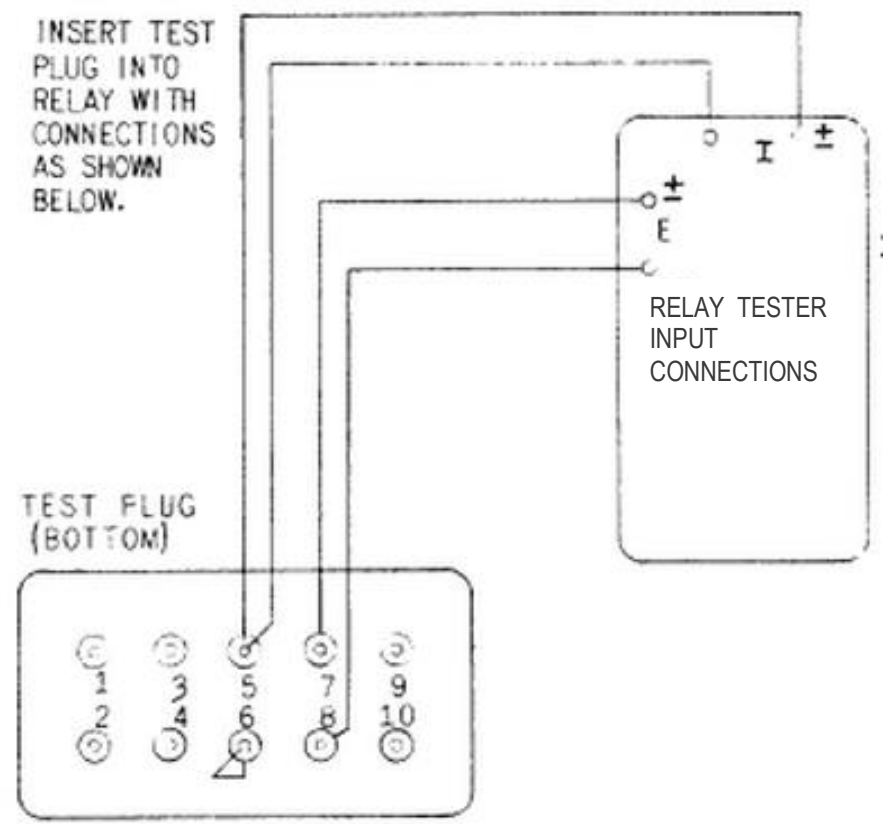

Figure 20: General Electric JBC51N overcurrent relay testing input connections diagram. Source: General Electric ${ }^{10}$

The JBC51N user manual provides a diagram for relay element connections for the JBC51N relays in the laboratory (Figure 21).

${ }^{11}$ Pulsar relay testing unit, AVO-MultiAmp Corporation, http://www.biddlemegger.com/biddleug/Pulsar_UG.pdf 


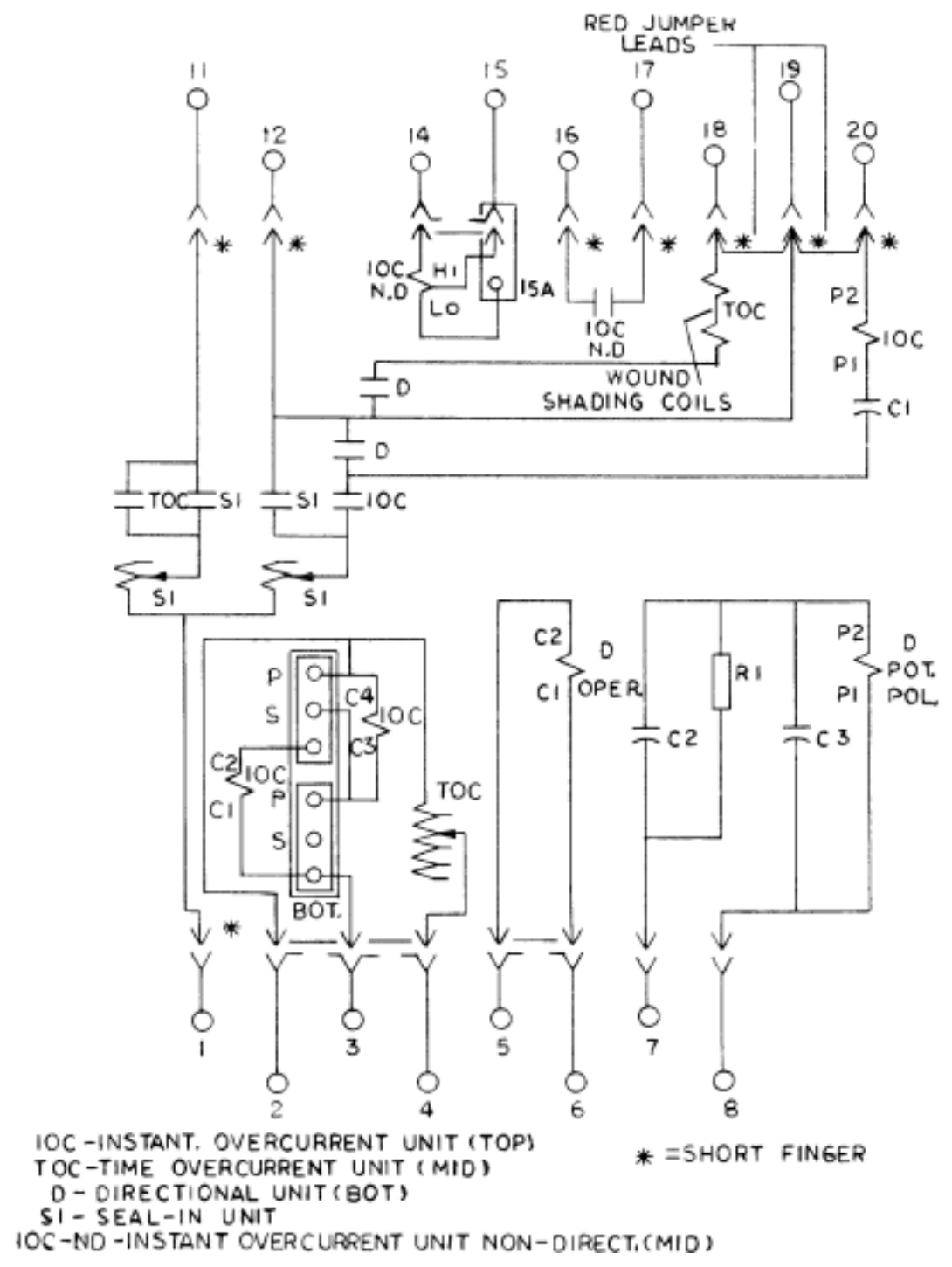

Figure 21: General Electric JBC51M (-) Y1A relay tesing connections. Source: General electric ${ }^{10}$

The second part of the assignment requires students to test the directional element of the SEL-351 digital relay. The relay manual is downloaded from the PSU desire-to-learn website and the following equipment is used,

- SEL-351 Protection System relay

- SEL-AMS 
- SEL-5401

- AcSELerator QuickStart software

- C724 ribbon cable

- C234A serial port cable

- C662 USB to serial port cable

To synthesize course lecture material, two of the three cases of tested fault data are from the lecture homework assignment. These values are programmed in the SEL-5401 software to test the relay DO element operation. The intention is to apply the results of the relay operation to the calculated results from student homework. Case 1 is a balanced 3- $\varphi$ fault (Table 5). Case 2 (Table 6) and case 3 (Table 7) correspond with the lecture course homework fault values.

The settings for the SEL-351 relay, programmed in the AcSELerator QuickStart software, are provided at the end of the written lab assignment instructions as are the simple logic variables. The students are required to record operation values and compare them to the calculated values from the homework detailed in their assignment report. 
Table 5: Case 1 fault parameters

\begin{tabular}{|c|c|c|}
\hline Quantity & $\begin{array}{c}\text { Magnitude } \\
\text { (Primary } \mathbf{k V} \text { and } \\
\text { amps) }\end{array}$ & Angle (degrees) \\
\hline $\mathrm{V}_{\mathrm{A}}$ & 13.2 & 0 \\
\hline $\mathrm{V}_{\mathrm{B}}$ & 13.2 & -120 \\
\hline $\mathrm{V}_{\mathrm{C}}$ & 13.2 & -90 \\
\hline $\mathrm{I}_{\mathrm{A}}$ & 3323 & 150 \\
\hline $\mathrm{I}_{\mathrm{B}}$ & 3323 & 30 \\
\hline $\mathrm{I}_{\mathrm{C}}$ & 3323 & \\
\hline
\end{tabular}

Table 6: Case 2 fault parameters

\begin{tabular}{|c|c|c|}
\hline Quantity & $\begin{array}{c}\text { Magnitude } \\
\text { (Primary kV and } \\
\text { amps) }\end{array}$ & \\
\hline $\mathrm{V}_{\mathrm{A}}$ & 16 & -6 \\
\hline $\mathrm{V}_{\mathrm{B}}$ & 76 & -120 \\
\hline $\mathrm{V}_{\mathrm{C}}$ & 76 & 120 \\
\hline $\mathrm{I}_{\mathrm{A}}$ & 4389 & -80 \\
\hline $\mathrm{I}_{\mathrm{B}}$ & 40 & -84 \\
\hline $\mathrm{I}_{\mathrm{C}}$ & 40 & -84 \\
\hline
\end{tabular}


Table 7: Case 3 fault parameters

\begin{tabular}{|c|c|c|}
\hline Quantity & $\begin{array}{c}\text { Magnitude } \\
\text { (Primary kV and } \\
\text { amps) }\end{array}$ & Angle (degrees) \\
\hline $\mathrm{V}_{\mathrm{A}}$ & 21.4 & -8 \\
\hline $\mathrm{V}_{\mathrm{B}}$ & 76 & -120 \\
\hline $\mathrm{V}_{\mathrm{C}}$ & 76 & 120 \\
\hline $\mathrm{I}_{\mathrm{A}}$ & 1238 & 98 \\
\hline $\mathrm{I}_{\mathrm{B}}$ & 24 & 109 \\
\hline $\mathrm{I}_{\mathrm{C}}$ & 31 & 111 \\
\hline
\end{tabular}




\section{Equipment Design}

The outline for the type of equipment necessary for the laboratory assignments to correspond to the lecture material was provided by the course instructor. In the first phase of laboratory development the equipment design and specifications expanded on this outline to develop the basic tools of. The second phase of equipment design happened during execution of the laboratory in response to laboratory needs. The third phase of equipment design involves the creation of a model-scale power system that was configurable for both radial and looped systems.

\subsection{Phase 1 Design}

For this first phase of equipment design, an outline of basic equipment needs based on the lecture material was provided by the lecture professor. The lecture course instructor, an industry professional with over thirty years of teaching experience in the field, specified the types of EM and digital relay curves which would be acceptable for the lab as well as his expectations for the laboratory equipment to properly supplement his course material. Appendix $\mathrm{C}$ lists the CAD drawings for the equipment designed for Phase 1, including relay rack drawings for the digital relay equipment.

\subsubsection{Electromechanical (EM) Relays}

Of the listed relays it was discovered that PSU was already in possession of the requisite amount of EM OC relays made by General Electric. These relays were of the IAC model line and have either an US Standard very inverse curve (U3) (Figure 13) or a US Standard extremely inverse curve (U4) (Figure 14). These curves belong to the IAC53 
and IAC77 relays, respectively, used in the fourth laboratory assignment testing EM OC relays.

The auxiliary relays of the third lab assignment were specifically requested by the lecture professor due to their plunger style construction. These relays consist of a single instantaneous overcurrent current or over-voltage relay and a number of contacts controlled by the operation of the instantaneous unit. Both the SV and SC use an electromechanical plunger relay, as illustrated in Figure 6. Each type had the same operation, with current or voltage applied to the coil to produce flux, moving the plunger. Due to their simplicity, testing auxiliary relays allowed students to easily become familiar with the MultiAmp relay testing instruments and learn general concepts of relay testing and operation. These relays were purchased from the eBay website.

The models of the EM Distance Overcurrent relays (DOR) were outlined by the course professor. Referencing PGE Foundation Power Laboratory EM relays, one DOR was found to be of an appropriate type, a JBC51N. Since the laboratory requires enough equipment for three workstations, two more of the same DOR relays were purchased. Again, eBay provided the least cost solution for these relays. These relays operate on the same induction disc, overcurrent element as the IAC relays, but this induction disc is also controlled by a DO unit, which is made up of a magnetic core and poles which sense the direction of the current and operate under a specified direction setting. When the DO unit contact is made, the time over current element is activated. 


\subsubsection{Fuse Holder}

The thermal properties assignment required specifying wire types for the heating experiment and the fuse damaging experiment. Since the wires were being heated at currents exceeding 20 Amps, it was necessary to design a fuse holder for safety and to provide secure connections for the current source. Grade B16 alloy steel threaded rod of 1/4"-20 thread were used to bolt the fuse holder walls and base together with zinc-plated steel acorn nut with 1/4"- 20 thread size, and a basic aluminum tube with dimensions of 9/32" OD, .2533" ID to cover the threaded rods providing a finished look to the product.
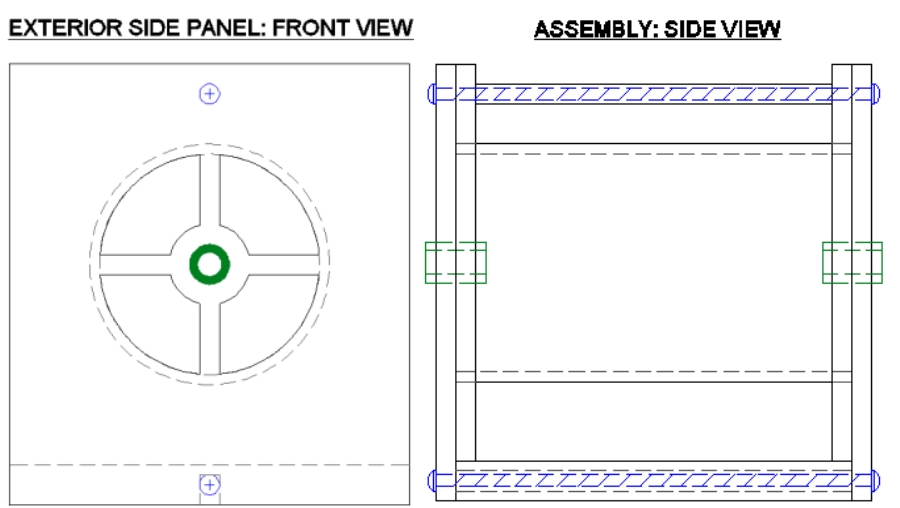

BOTTOM PANEL: UNDERSIDE VIEN

Figure 22: Fuse holder design. Source: Ferris

The body of the fuse holder in Figure 22 was made of 0.25 inch thick acrylic layers bonded together, with ample venting spaces at each end to approximate free air conditions. The fabrication was done with a laser cutter by Etchpop, a local company founded by a colleague, Chester Lindgren. The thickness of the acrylic walls and base was 0.5 inches. Ceramic tube inserts provide insulation between the acrylic material of the walls and the bare wire being heated by the current source (Figure 22) and were 
donated by from Ceramic Technologies Inc. These insulating tubes are an alumina material with a maximum use temperature of 3000 degrees.

Regular flat ceramic washers, of size no. $1 / 4$ " -20 , insulation for the $1 / 4$ "-20 inch fastener bolts, were specified as and fabricated by Ortech Inc., a ceramic parts manufacturer located in Sacramento, CA. These ceramic discs provided insulation between the acrylic walls of the holder and the $1 / 4$ "-20 size galvanized steel flat washers used to secure the copper conductor around the bolts and provide a current path for testing. A Pyrex tube with an outer diameter of three inches and a length of five inches spans the distance between acrylic wall layers. It is held in place with a combination of the acrylic walls and the threaded rod, to provide further protection from the exposed conduit used in the experiments. During the fuse testing experiment, small gauge wire is heated to its melting point and it is necessary to have the material contained for safety. A small hole was blown into the Pyrex tube wall by the PSU chemistry department glass lab for students to be able to position the thermocouple on the center of the wire for the first part of the Thermal Properties laboratory assignment.

\subsubsection{Electromechanical Relay Testing Systems and High Current Source}

To test the EM relays and to be able to provide a current source for the thermal properties laboratory assignment and the CT testing assignment, high current source relay testing units were required. At least three testing modules were needed, one for each learning station. Due to the prohibitive costs of contemporary testing equipment with a high 
current sources, outmoded testing units were sourced. The models are part of the history of the electric grid, and are able to test the EM equipment accurately. They also provide current sources and some provide voltage sources. Two models that provide both voltage and current, combined on single phase outputs and with dependent operation were found on eBay. These were the MultiAmp testing models SR-51A andSR-51S, and SR-76A were procured from eBay sellers as previously used items. The SR-51A and SR-51S models only differed by clock interface style and used the same manual as a result. The SR-51S was analog timing and the SR-51A was updated to digital timing. Contacting Megger, formally MultiAmp, revealed that the user guide for the SR-51A model was the only manual available for the SR-51 model series was the SR-51A reference. Otherwise the systems are the same, providing the ability to generate both a current output and a voltage output simultaneously for tests on a single electrical phase. These units were rated to able to produce currents at levels of $140 \mathrm{~A}$ to $200 \mathrm{~A}$ for 20 minutes. This high current range was necessary for the thermal properties lab and the CT testing lab, since the highest ratio of CT was 100:5 and the CT had to be pushed into saturation by increasing the primary current to at least $120 \%$ of the nominal primary value.

The SR-76 module was also purchased on eBay and is the main current source component of a set. It had no voltage output capability on the half of the set purchased for the lab and also only tests one phase of the relays like the other SR series. For all of these MultiAmp relay models there was no ability to vary the voltage and current 
independently. It was discovered that without this ability, the DOR relays in laboratory assignment eight would not be able to be tested.

\subsubsection{Digital Protection Equipment}

Digital relay equipment was specified by the lecture professor to follow lecture course material. The initial request for the SEL donation listed a variety of relays that would cover overcurrent, transmission line protection, generator protection, and distance protection. This request included relays for future expansion of the laboratory curriculum, but exceeded the initial nine lab curriculum outline needs. Enough equipment for six stations was requested, and equipment for three laboratory stations was granted to the laboratory. These three stations worth of equipment resulted in being approximately $\$ 50,000$ of brand new laboratory equipment, specifically manufactured for the laboratory. Along with the donation came correspondence of interest in continued work with PSU in the future. The donated devices included,

- SEL-551 Overcurrent protection

- SEL-351 Transmission Line protection

- SEL-311L Distance line protection

- SEL-2400 Programmable Automation Controller

- SEL-3530 Real Time Automation Controller

- SEL-AMS relay testing system 
The SEL-AMS is the SEL specific relay tester used in conjunction with SEL-5401

software to simulate systems and test one relay at a time. The AMS does have the ability to test the pilot communications and operations of two SEL-311L relays, which provide distance protection on transmission lines.

The SEL-551, a simple overcurrent relay with only instantaneous (50) and time-inverse (51) overcurrent protection and breaker reclosing (79) capability and limited outputs, protects distribution substation equipment and other more simple, radial topology systems. In industry, this unit replaced EM relay models, such as the IAC53 relays and the auxiliary relays tested in the earlier laboratory assignments.

The SEL-351 relay is a total protection system for distribution system for industrial and utility feeder protection. This relay is used in the laboratory assignments for its DO capability (67), but the relay has the capability of many other protection elements such as overcurrent protection (50/51), overvoltage (59),

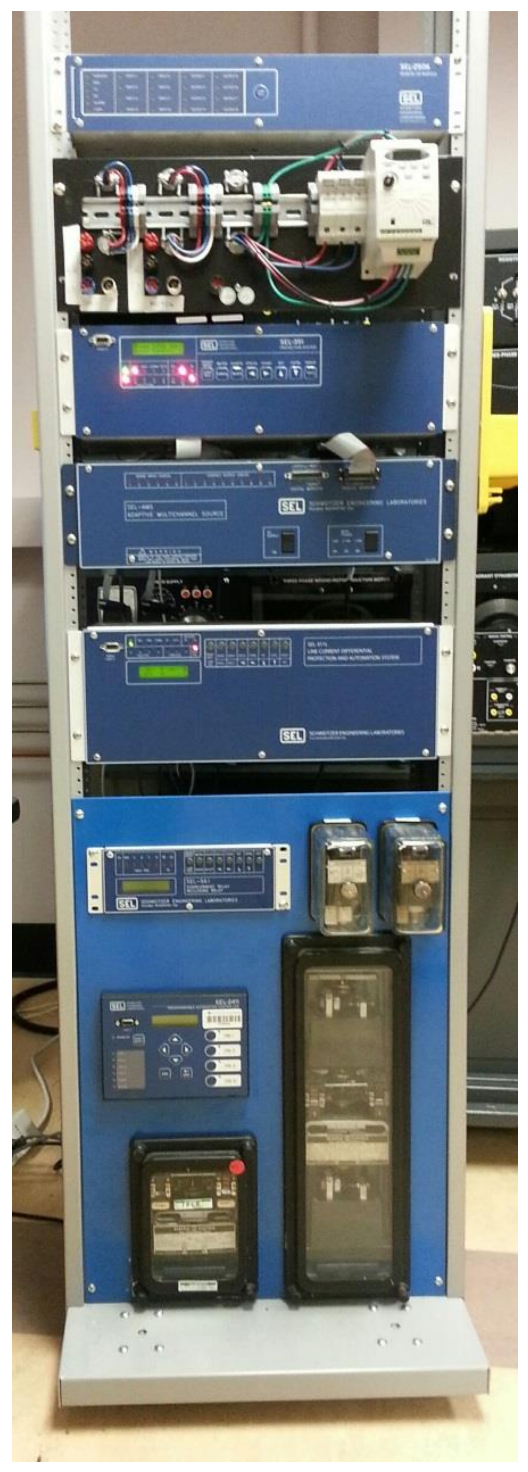

Figure 23: Laboratory station relay rack undervoltage (27), and directional power flow (32). This relay is one of the most 
common found in substations and switch yards to date. The extra functions on this relay open the possibility to expanding laboratory subject curriculum in future classes.

The SEL-311L relay is for distance protection of transmission lines, but similar to the SEL-351 it also holds much more capabilities than just distance protection (21). This relay has the capability to communicate with another SEL-311L relay by pilot wires for the use of the differential current (87) element. It can also protect for over- and underfrequency (81) conditions. While this relay was not used in these laboratory assignments, it provides expansion possibilities for future laboratories and student projects.

There are two pieces of equipment form the SEL donation which do not serve any protection functions, but which have control capabilities. The SEL-2400 Programmable Automation Controller (PAC) was not designed into this initial laboratory curriculum due to the fact it does not have any of the basic protection elements explored in this laboratory course, but it affords the ability to expand the laboratory in the future and introduces a level of control potential for projects. Similarly the SEL-3530 Real Time Automation Controller (RTAC) function lies beyond the current needs of the protection laboratory and also offers no inherent protection elements itself. Instead it is capable of configuring automation systems and collecting data in real time. 


\subsubsection{Relay Racks}

A housing structure was required to use and store the protection relays and other digital equipment in this laboratory therefore standard relay racks manufactured by Bud Industries were specified based on the size of the rack model (Figure 23). The RR-1367 series open relay racks have 77 inches available for mounting relays. This exceeded the current space needs determined by the final equipment list of Phase 1 made up of the digital SEL devices, the digital SEL relay tester, and the four EM relays but allowed for future expansion for the digital relay equipment. These racks were ordered with casters capable of supporting 250 pounds per caster.

\subsubsection{Relay Panel}

While the majority of the modern, digital relays from SEL were of the 19 inch rack mounting style, the SEL-551 and SEL-2400 both required a panel mount. These model dimensions were included in the panel designed to hold the EM relays, none of which are a standard 19 inch rack size. These older EM relays are all panel mount type relays, meaning that the rack style mounting of the digital relays would be insufficient. This need resulted in panels being designed and fabricated for all three stations (Figure 24). The weight of the EM relays required them to be oriented at the bottom of the racks, used as a ballast for the modular racks. 


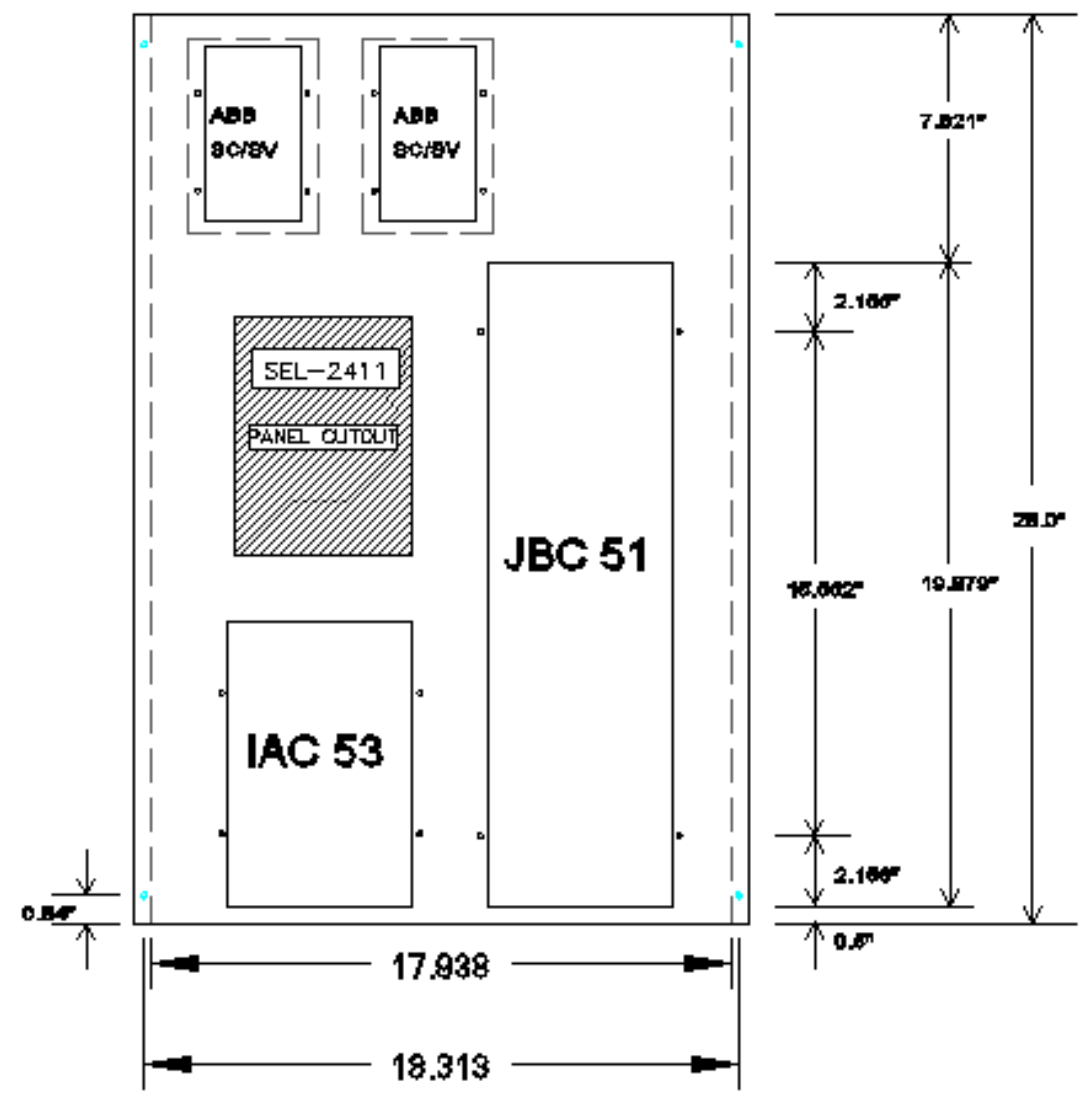

Figure 24: Relay panel drawing.

Source: Ferris

\subsubsection{Current Transformers}

Veris Industries generously offered to donate samples of any of their instrument transformers for our laboratory. Veris, a local energy sensor and control peripherals supply company in Portland, OR, had metering class relays of a small enough turns ratio to be able to experiment with overcurrent tests on the CTs since at least $120 \%$ of the rated primary amps had to be able to be applied at the rated burden 2.0 VA. These CTs had turns ratios of 100:5 and 50:5 of part numbers AL101 and AL500 respectively. Both CT models are solid core, and the AL101 has an accuracy tolerance of 1\% while the AL500 has an accuracy tolerance of 3\%. Both models have a rated burden of $2 \mathrm{VA}$ and both 
models are rated for their accuracy tolerance from $10 \%$ to $100 \%$ of rated current (Table 2).

\subsubsection{Rheostat Burden}

For the overcurrent and over-burden CT tests, a variable load was required that could handle at least 5 amps of current with a very small burden rating close to 0.08 ohms. To calculate the length of wire needed for a single layer of wire wrapping to meet the rated burden, table 1.2 on page 1-8 from the resource available at CED Engineering website for copper wire resistance per foot for different wire gauges ${ }^{12}$ was used, the ohms/1000 feet for solid 14 AWG copper was 2.97, which converts to $0.00297 \mathrm{Ohms} / \mathrm{ft}$. Since 0.08 Ohms, and given an approximate shaft diameter of 1.5 inches on the rheostat with a length of 5 inches, it was established that 27 feet of wire would need to be wound to meet the rated burned. For the $14 \mathrm{AWG}$ at a diameter of 0.064 inches, wrapping 27 feet required 4.32 inches of length available on the shaft to complete the 67.5 turns. With the wire connections on the rheostat considered in the length requirements of the rheostat, the 4.32 inch length of wire wrap along the shaft exceeded the usable space since the rheostat can have only one layer of wire wrapping.

At 16 AWG, a linear resistance of 0.00473 ohms per feet, and 0.051 inch diameter only 17 feet of wire to be wrapped 42.5 times around the rheostat shaft and was calculated to be 2.17 inches of wrap length on the shaft. These dimensions suited the size restrictions

\footnotetext{
${ }^{12} \mathrm{~A}$. Bhatia, Electrical Conductors Course, CED Engineering, http://www.cedengineering.com/upload/Electrical\%20Conductors.pdf
} 
of the rheostat. An undergraduate was hired to wrap the rheostats with a lathe. The layer of wire insulation covering the conduit was then peeled off along the wrappings where the rheostat contacts connect to the conductor to adjust resistance. This way the upgraded rheostats provided $0.08 \mathrm{ohms}$ of resistance.

\subsubsection{Oscilloscopes and Current Probes}

Tektronix and Test Equity worked together to donate six, 2000 amp current probes and provide a fifty percent educational discount on six Tektronix TPS2012B oscilloscopes as the least cost option signal monitoring. The high current rating on the probes was the only option fulfill the laboratory need for measurement equipment rated at greater than 100 amps in order to monitor the primary current in the CT testing lab of overcurrent response for the AL101 series relays with 100:5 amp turns ratio.

\subsection{Phase II Design}

During the term slight equipment adjustments had to be made in order to be able to test the EM DORs. As a result an Avo Pulsar relay tester was sourced from AccuSource Electronics, who provided a generous educational discount of $\$ 10000$ off the unit price for the laboratory. This relay tester was a more modern unit than the MultiAmp units used in the assignments prior to the DOR testing; there were three digital cards present for both voltage and current supplies allowing all three phases of a relay to be tested and allows for voltage and current to be varied independently, and even has phase angle control. These features make the Pulsar adequate for testing directional element of the DOR relay. 
One minor operation issue was recognized during this phase of the laboratory. This was related to the discontinuity in the JBC manual for relay port connections compared to the ports available on the physical JBC51N model itself. In the JBC manual, relay models 51M-Y1A were given a blanket diagram (Figure 21) that contains too many ports for the laboratory JBC51N relay models. It was necessary to partially dismantle one of the JBC51N relays to determine the proper wiring of the unit for testing. Otherwise all other equipment needs were adequate for the course term.

\subsection{Phase III Design}

The Phase I and Phase II design portions of the laboratory created a solid base for introducing students to applications of the lecture course theory. Phase 3 was designed so students could use the digital relay equipment inside a scaled model of a 208 volt, 2.8 kVA modular power system with the capability to imitate load shifts and phase imbalances. This system was designed for both radial and looped systems with two sources feeding into the Western Electrical Coordination Council territory of the electric grid. The entire system consisted of three buses with variable line lengths between each. Each bus had a relay rack dedicated to the bus specific generation, load and transmission lines along with circuit breakers. Panel faces were equipped with binding posts to provide secure, color coded connections for each phase. 


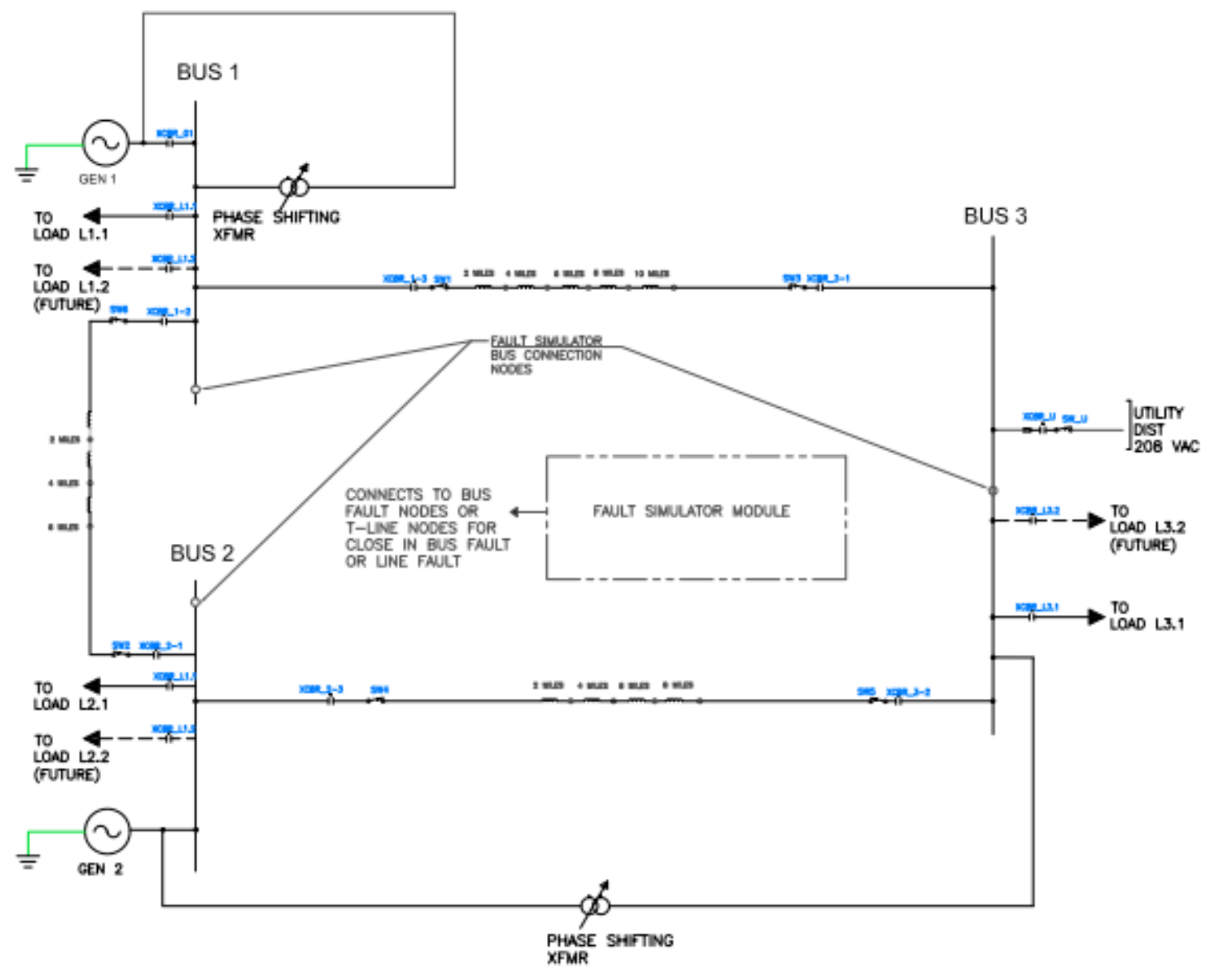

Figure 25: Phase III system one-line drawing.

Source: Ferris

\subsubsection{Generation}

The power source for the system was a challenge due to the size restrictions of the model and the physical space available in the laboratory classroom. Ideally a generator-motor set would be used to model the system source but the size restrictions of this model system made the use of small rotating machines not feasible due to their prohibitive cost and scarcity. Using the Georgia Tech laboratory model [18], the same National Instruments NI 6722 waveform generator was specified to be used in conjunction with 
the National Instruments LabView software educational suite as the generation control for phase voltage amplitude and system frequency.

As with the Georgia Tech lab design, a seven channel Sunfire TGA 7401 audio amplifier capable of 400 Watts per channel for an 8 ohm load was specified. [18] Each phase was sourced by two channels, with the exception of the neutral phase which only used one channel. These channels were designed to provide a combined 800 Watts to each of the three phases. Each channel of the Sunfire amplifier had a maximum capability of 56 Vrms output, so each channel was specified to be sent through a bank of transformers designed similarly to ones used in the Georgia Tech power system to boost the system voltage to 120 volts (Figure 26). [18] This resulted in a system line current of 6.7 amps.

INPUT: NI 6722 WAVEFORM GENERATOR TO 7 CHANNEL SUNFIRE TGA 7401 AMPLIFIER

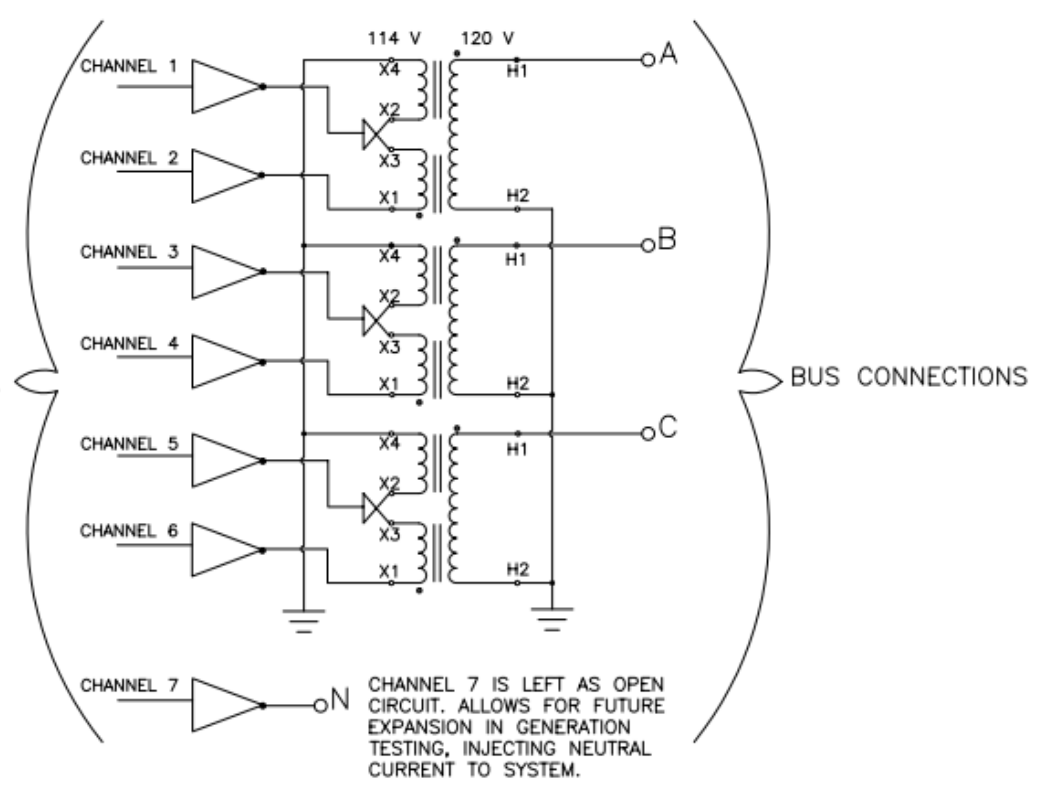

Figure 26: Generation transformer bank drawing. Source: Ferris 


\subsubsection{Generation Transformers}

Since the NI 6722 waveform generation board and amplifier generation set produce a maximum voltage of 56 volts per channel, a bank of isolation transformers was designed using a modified design from the Georgia Tech model-scale power system (Figure 26). [18] This bank of transformers, with a parallel connections on the low voltage side, boosts the voltage level to 114 volts per phase. This bank consists of three, 1:1 turns ratio isolation transformers which can be connected in either parallel or series configurations on the low-voltage terminals. For this application a parallel connection was used to combine the dual amplifier outputs needed for each phase.

\subsubsection{Transmission Lines}

The transmission line modules were designed with four sections of wire wrapping for the three power phases plus the neutral phase of the lines wrapped around a four inch diameter acrylic core. It was given that there are 150 turns for each phase and the neutral phase, of 14 AWG and 16 AWG respectively. Taking into account the circumference of the module bobbin at four inches and the average diameter of $14 \mathrm{AWG}$ at 0.0642 inches and $16 \mathrm{AWG}$ at 0.0508 inches an online wire coil physical properties calculator from Daycounter, Inc. Engineering services ${ }^{13}$ was used to find the required amounts of wire for each gauge.

\footnotetext{
${ }^{13}$ Coil Physical Properties Calculator, Daycounter, Inc. Engineering Services, http://www.daycounter.com/Calculators/Coil-Physical-Properties-Calculator.phtml
} 
For the three phases the 14 AWG magnet wire was specified from an $11 \mathrm{lb}$ spool made up of 870 feet. At 150 turns and a diameter of 0.0642 inches, the online calculator found that each of the phases will use 184 feet, making a total of 552 feet per bobbin. There were three bobbins in one 2-mile section of transmission line which required 1656 feet per 2-mile section. There are a total of twelve 2-mile sections in the system which required approximately 20000 feet of 14 AWG copper wire to model the 24 combined miles of system transmission line.
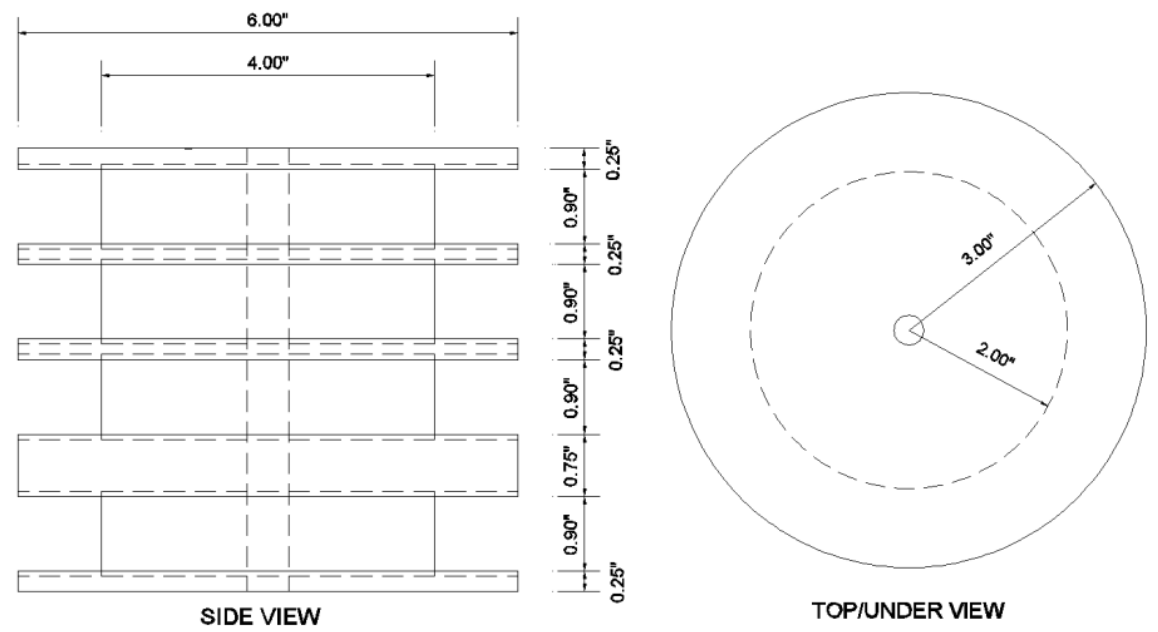

Figure 27: Transmission module plan drawing.

Source: Ferris

The Georgia Tech laboratory specifies this transmission line module and also includes a capacitor board to model parasitic capacitance of a transmission line. [18] In the future the same design will be utilized from the Georgia Tech model, since the systems are of the same rating and similar design and Georgia Tech was able to design these specific parameters using specialized software not accessible to PSU. [18] The transmission impedance parameters are given as, 
Table 8: Transmission line module impedance parameter values [18]

\begin{tabular}{|l|l|}
\hline Line Parameter & Measurement \\
\hline $\mathrm{L}_{\mathrm{s}, \mathrm{a}}$ & $3.44 \mathrm{mH}$ \\
\hline $\mathrm{L}_{\mathrm{s}, \mathrm{b}}$ & $3.39 \mathrm{mH}$ \\
\hline $\mathrm{L}_{\mathrm{s}, \mathrm{c}}$ & $3.44 \mathrm{mH}$ \\
\hline $\mathrm{L}_{\mathrm{s}, \mathrm{n}}$ & $3.36 \mathrm{mH}$ \\
\hline $\mathrm{L}_{\mathrm{m}, \mathrm{ab}}$ & $1.619 \mathrm{mH}$ \\
\hline $\mathrm{L}_{\mathrm{m}, \mathrm{ac}}$ & $0.751 \mathrm{mH}$ \\
\hline $\mathrm{L}_{\mathrm{m}, \mathrm{an}}$ & $0.321 \mathrm{mH}$ \\
\hline $\mathrm{L}_{\mathrm{m}, \mathrm{bc}}$ & $1.631 \mathrm{mH}$ \\
\hline $\mathrm{L}_{\mathrm{m}, \mathrm{bn}}$ & $0.586 \mathrm{mH}$ \\
\hline $\mathrm{L}_{\mathrm{m}, \mathrm{cn}}$ & $1.213 \mathrm{mH}$ \\
\hline $\mathrm{R}_{\mathrm{a}}$ & $0.492 \Omega$ \\
\hline $\mathrm{R}_{\mathrm{b}}$ & $0.489 \Omega$ \\
\hline $\mathrm{R}_{\mathrm{c}}$ & $0.495 \Omega$ \\
\hline $\mathrm{R}_{\mathrm{n}}$ & $0.740 \Omega$ \\
\hline
\end{tabular}

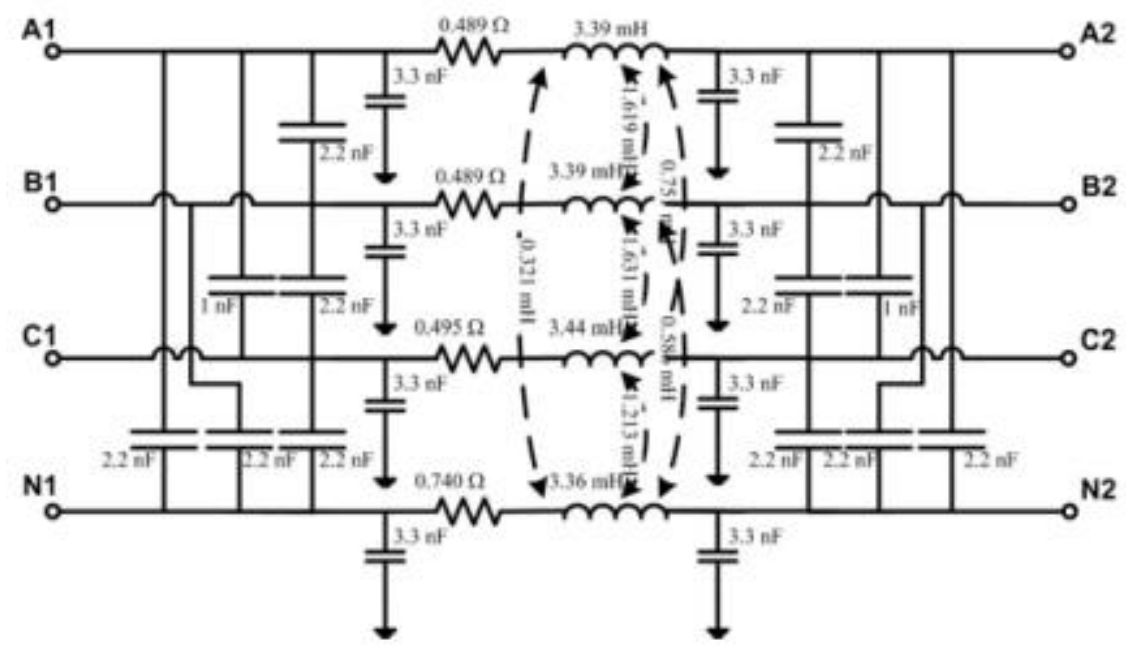

Figure 28: Transmission line model.

Source: Mohagheghi [18] 


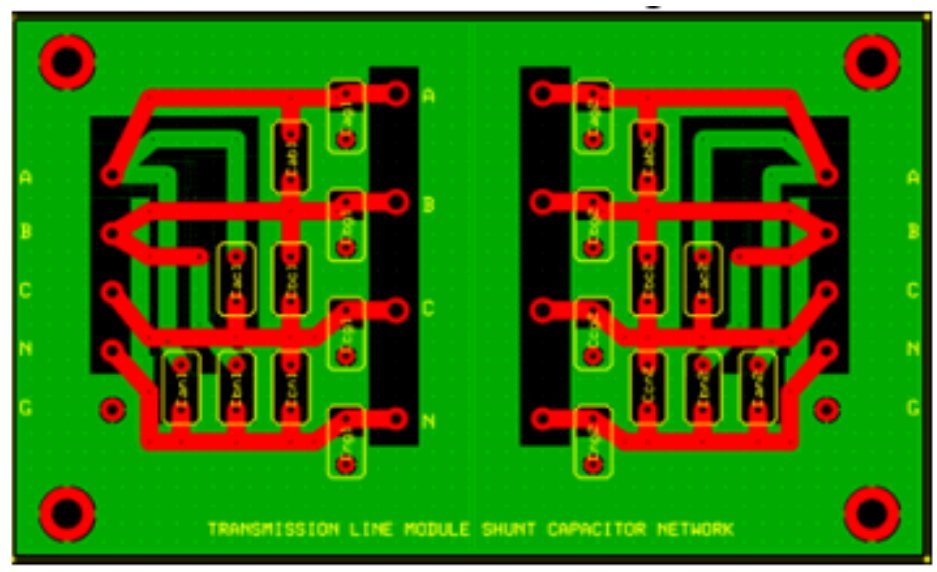

Figure 29: PCB board design.

Source: Mohagheghi [18]

\subsubsection{Load Shifting Transformer Bank}

The load shifting bank was made up of a combination of three single phase isolation transformers and single phase variable transformers. The Georgia Tech model for causing load imbalances was used for the design, where the primary terminals of the isolation transformers are connected in series with the 120 volt transmission line. The secondary terminals of the isolation transformer were connected to the primary terminals of three variable transformers in a variable configuration, powering the variable transformer at the nominal 120 volts. The secondary terminals of the variable transformer bank connected to the transmission to allow an injection of the line-to-line voltage to be injected into the system at a load bus to simulate a load imbalance (Figure 30) (Figure 31). 


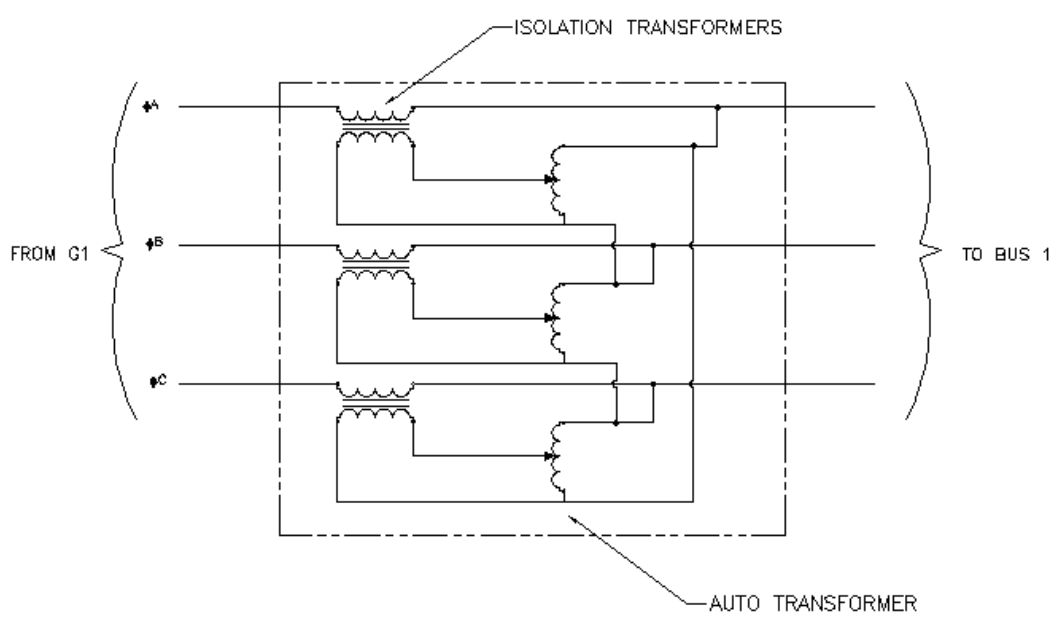

Figure 30: Load shifting transformer bank wiring diagram. Source: Ferris

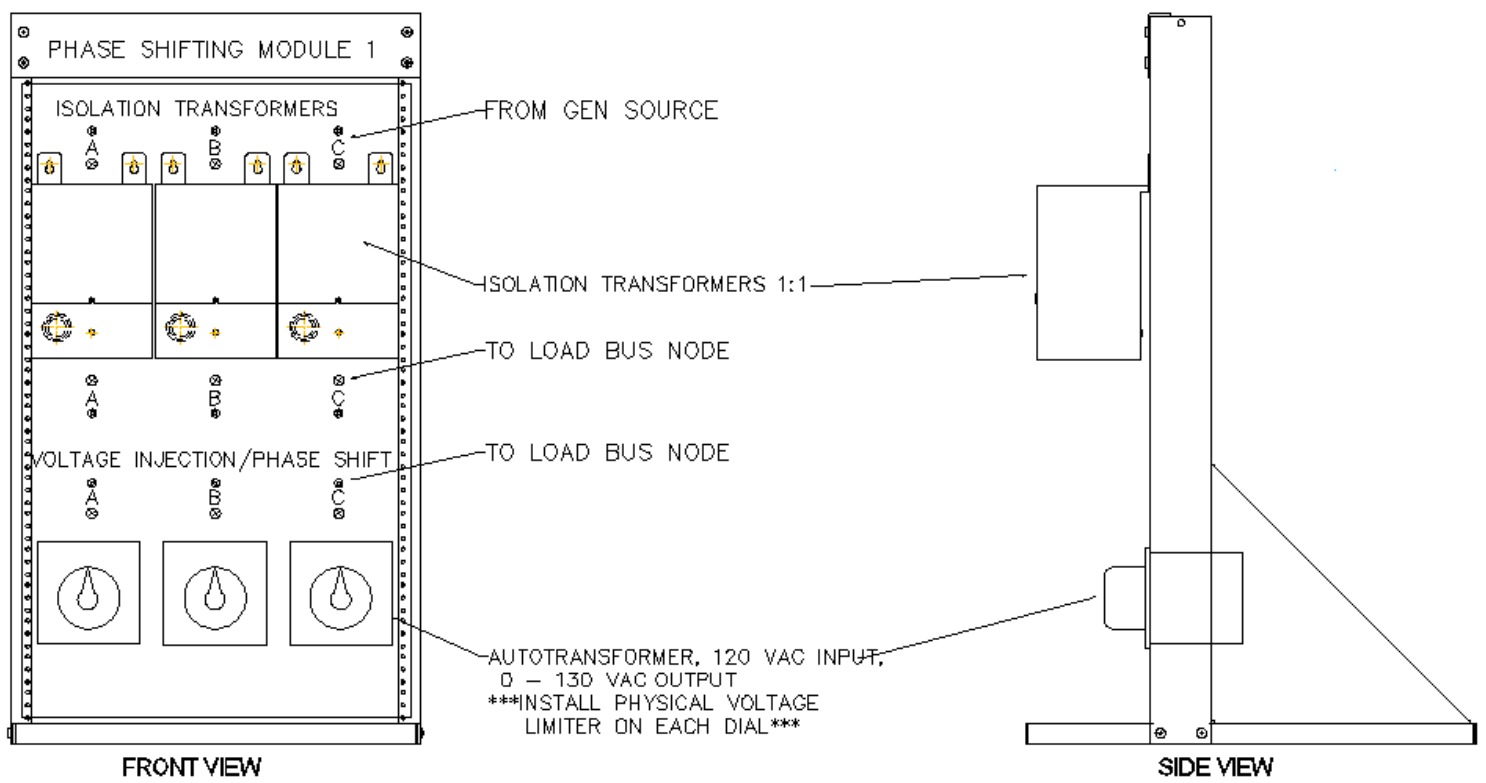

Figure 31: Load shifting transformer bank plan drawing. Source: Ferris

The system was designed to be manually operated per phase with the use of the autotransformer faceplate dials. The plan drawing includes a note regarding the need for 
a physical voltage limiter to be installed on each autotransformer dial to prevent damaging amounts of voltage to be accidentally sourced into the system during use.

\subsubsection{Bus, Generation, and Load Panels}

A combination of $1 \mathrm{U}, 2 \mathrm{U}, 3 \mathrm{U}$, and $4 \mathrm{U}$ blank relay rack panels were used to create the physical interfaces of generation, transmission lines, buses, and loads for the system electrical connections. The panels were designed to mount the proper circuit breakers on DIN rail, represented by standard octal pin relays, and had binding posts for the electrical system connection ports and relay protection control ports. The components on the panels were designed to be bonded to ground for safety.

The $4 \mathrm{U}$ panels are only used for the To Bus 3 panel on Bus 1 and the From Bus 1 panel on Bus 3 because of the number of circuit breakers needed to represent the single phase switching of breakers along the ten mile line. The multiple protection control ports required for circuit breaker operation on all four lines also required more physical space than the $3 \mathrm{U}$ panels were capable of providing. Four 8-pin DPDT were designed to operate the switching of each electrical phase separately, a common configuration in long distance line protection for fault clearing. In industry only longer lines have single pole switching which is why the ten mile line of the system is the only line with this type of protection. 
The $3 \mathrm{U}$ size panels were designed for the rest of the equipment panels related to generation, loads, and buses and included the same binding post ports for the electrical interconnections and relay protection control. These panels had only two relays for threephase circuit breaker switching, one 11-pin octal 3PDT and one 8-pin DPDT octal with parallel coils for simultaneous operation of all three phases and neutral under fault conditions.

The binding posts used for the electrical interconnections were specified to be color coded for the three phases, neutral, and ground connection ports were specified for the panels. These binding posts are capable of carrying 15 amps and are black, red, blue, white, and green following the U.S. standard for three phase electrical systems. The components on the panels were designed to be bonded to ground for safety.

\subsubsection{Circuit Breakers}

System circuit breakers were designed to be simulated by octal pin relays mounded on DIN rail on the panel faces for generation, buses, and loads. For the generation and loads, single pole tripping was designed with a 3PDT, 11-pin octal relay on the three phases electrically paralleled with a 2PDT, 8-pin octal relay coil on the neutral line to open all phase circuit breakers under fault conditions. All relays were Form $\mathrm{C}$ contact rated at 10 amps on the contacts and coil voltages of $125 \mathrm{VDC}$ to be able to respond to the 125 VDC control voltage from the digital SEL relays. The relay contacts were rated for 240 VAC. 
The double throw feature on these relays allowed for the design of a separate trip coil and close coil command from the digital relays, this way the circuit breakers would not reclose until they are reset by the relay itself. This type of control was designed because of its standard use in industry applications as shown in the SEL relay user guide examples of standard relay configurations. ${ }^{14}$

\subsubsection{System Racking}

The same relay racks were used to provide the backbone for Phase 3 as for the digital and EM relays in Phase 1 (Figure 23). This design makes the racks completely modular. The same 77 inch tall relay racks used in Phase One for the protective relay equipment were used for the system racking. Their modular capability allowed a flexible application of design configurations for radial and looped systems.

These racks were large enough to house the entire system backbone, including the transmission line modules. The manufacturer of these racks also makes rack shelving, which was employed in the design for stacking and storing the two mile sections of the transmission line modules.

${ }^{14}$ SEL-551 Instruction Manual, Schweitzer Engineering Laboratories, www.selinc.com 


\section{Assessment and Evaluation}

The effectiveness of the laboratory was assessed using both qualitative and quantitative analysis. Since graduate student participation in the laboratory was voluntary while the undergraduate participation was required as part of the lecture grading and some teams had blended graduate levels, the undergraduate and graduate students were assessed together. A short lab report detailing assignment results was due weekly, as specified in the written assignment instructions. To qualitatively address the effectiveness of the laboratory the course was assessed in two ways; 1) using weekly student surveys for each individual lab; and 2) assessing the overall effectiveness of the individual assignment goals using an end-of-term quiz. This end-of-term quiz assessed how well the course followed the emphasized overarching laboratory objectives adopted from Feisel's thirteen objective outlining the fundamental purpose of educational laboratories in engineering. [11] [12]

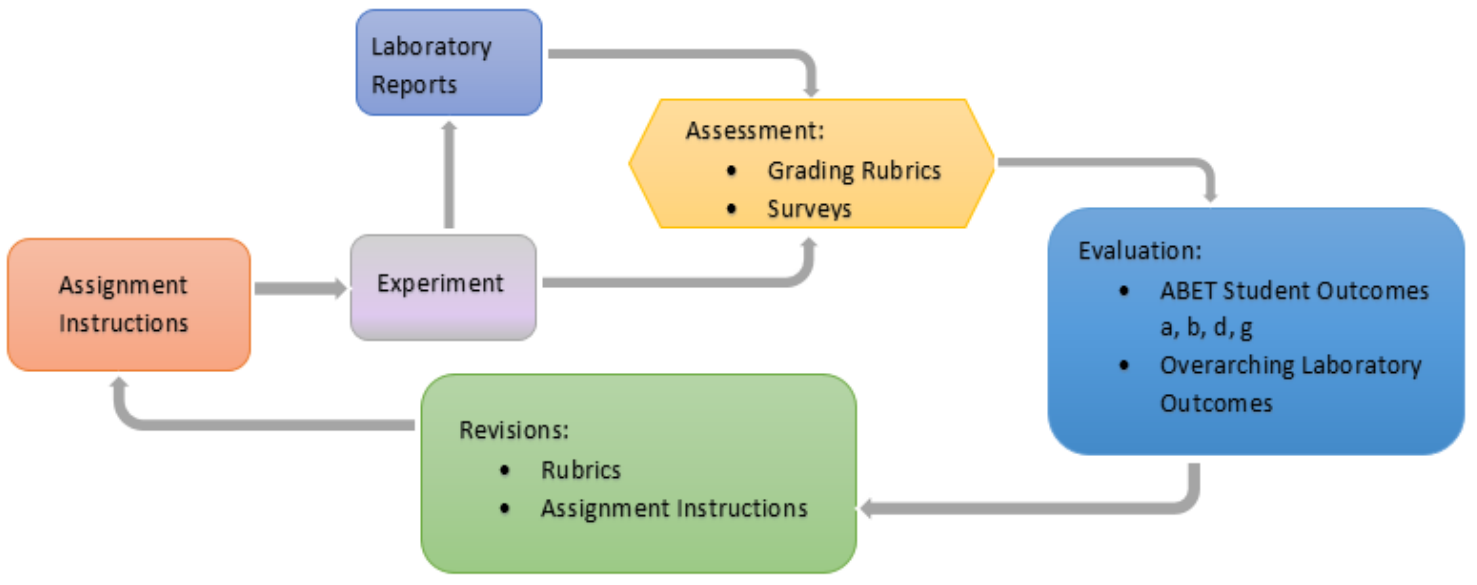

Figure 32: Assessment and Evaluation Feedback Cycle. Source: Ferris 
The results of the rubrics and surveys guided the revision of the rubrics and assignment instructions to improve on outcome achievement. This cycle of assessment and evaluation is illustrated in Figure 32.

\subsection{Measures Used}

A combination of grading rubrics and student participation surveys were used to assess and evaluate the efficacy of the outcomes of the laboratory and the objective of the individual laboratory assignments.

\subsubsection{Rubric Assessment of Weekly Reports}

The weekly reports were used to measure student understanding of assignment material quantitatively for ABET student outcomes (a), (b), (d), and (g). These objectives are described by ABET as,

a. an ability to apply knowledge of mathematics, science, and engineering;

b. an ability to design and conduct experiments, as well as to analyze and interpret data;

d. an ability to function on multi-disciplinary teams;

g. an ability to communicate effectively.

Assignment reports were evaluated for this study on a plus (+), check $(\checkmark)$, minus (-) scale for this assessment where $(+)$ and $(\checkmark)$ results considered the outcome achieved, where (+) indicates the students exceed expectations, a $(\checkmark)$ indicates they meeting expectations and, and (-) indicated they fail to meet expectations. Outcomes (a), (b), and (g) were 
associated with the written communication of the understanding of the engineering and mathematic principles of the experiment, data analysis, and the presentation of reports. Outcome (b) was assessed separately for the assigned tasks, through the physical execution of individual assignment objectives associated with the assignment experiments. Outcome (d) was assessed separately for the effectiveness of teamwork within the small groups.

\subsubsection{Assignment Outcomes and Overall Laboratory Outcomes Surveys}

Each individual lab assignment states the lab outcomes at the beginning of the written instructions. Weekly quizzes were created and posted on the university distance learning site, D2L, and were of voluntarily based participation. Questions regarding the effectiveness of the laboratory in meeting each individual objective were measured in multiple choice form. Each quiz also had a short answer portion requesting student feedback about the laboratory based on student experience.

At the end of the term, a survey assessing the ten overarching laboratory outcomes [12] was made available to the students via D2L and was also voluntarily based participation. This survey had the same format as the weekly surveys, with each individual goal assessed in multiple choice form and a short answer portion at the end for comments regarding the overall lab itself. There was another short answer portion added asking students if making the lab a separate, one credit course would be preferable to having the laboratory attached directly to the lecture and only graded on a participation basis, which 
was required for the undergraduates and optional for graduate students. This survey was posted the last week of the term upon completion of all nine labs.

\subsection{Weekly Survey Assessment Data}

The weekly surveys allowed for a micro-level assessing the overall effectiveness of the laboratory assignment in meeting the laboratory assignment goals, as stated in the written laboratory instructions. Each objective was considered separately for student review based on student personal experience with each assignment. These responses assisted directly in alterations made to individual laboratory assignment instructions.

\subsubsection{Laboratory 1 - ASPEN Introduction Survey Results}

The first survey assessed student involvement with the ASPEN software introduction.

There were twenty-three survey responders to the first survey. Student survey participants answered four multiple choice questions regarding the assignment's effectiveness of meeting the stated goals on the written lab instructions. Students were given a final space to leave their comments regarding their experiences in the lab, both positive and negative. As a result specific aspects of the laboratory experience were detailed by students. The four questions used for assessment were,

- Did this lab meet the stated objective of creating simple cases in ASPEN OneLiner, including positive-, negative- and zero-sequence impedances of line and generators as well as proper transformer connections? 
- Did this lab meet the stated objective of performing basic analysis of the results of the Power Flow function of the ASPEN software on the radial and looped systems?

- Did this lab meet the stated objective of identifying the results of the power flow that have an influence in the fault study?

- Did this lab meet the stated objectives of performing a basic fault study, obtaining results for three-phase, single line-to-ground, line-to-line, and line-toline-to-ground faults in the relevant parts of the system and interpret the results?

\subsubsection{Laboratory 1 - Response to Survey Questions}

Of the twenty-three survey participants, twenty-one of the responders documented that they "learned more than expected" with regard to Question 1, modeling simple cases in the ASPEN software with proper sequence components of line and generator and proper transformer connections. Two students answered "Yes.". There were zero responses of "Kind of..." and "Not really" indicating students who responded to this assignment survey considered this objective either met or exceeded.

For the objective regarding basic analysis of the ASPEN results for both the radial and looped system models nineteen of the twenty-three student sample "Learned more than expected" while two students responded "Yes" and two responded "Kind of..." Again, there were zero responses of "Not really". 
The third objective addressed student assimilation and application of knowledge from previous power systems analysis classes in the identification of results that affect the fault study. the identification the parts of the power flow results that have an influence in the power study had the weakest response, where fifteen participants responded "Learned more than expected", three students responded "Yes", and five students responded "Kind of..." Zero participants responded "Not really".

For the final objective of the assignment regarding performing a basic fault study and obtaining results for three-phase, single line-to-ground, line-to-line, and line-to-line-toground faults in the relevant parts of the system, nineteen of the twenty-three students "Learned more than expected", three students responded "Yes", and one survey participant responded "Kind of..." For the twenty-three survey responders, this gave a $96 \%$ positive response for this objective and zero responses of "Not really". It is notable that all students participating in this found that every objective for the first laboratory assignment was met to some extent, as evidenced by the absence of responses of "Not really" for all four objectives. 
Learned more than expected

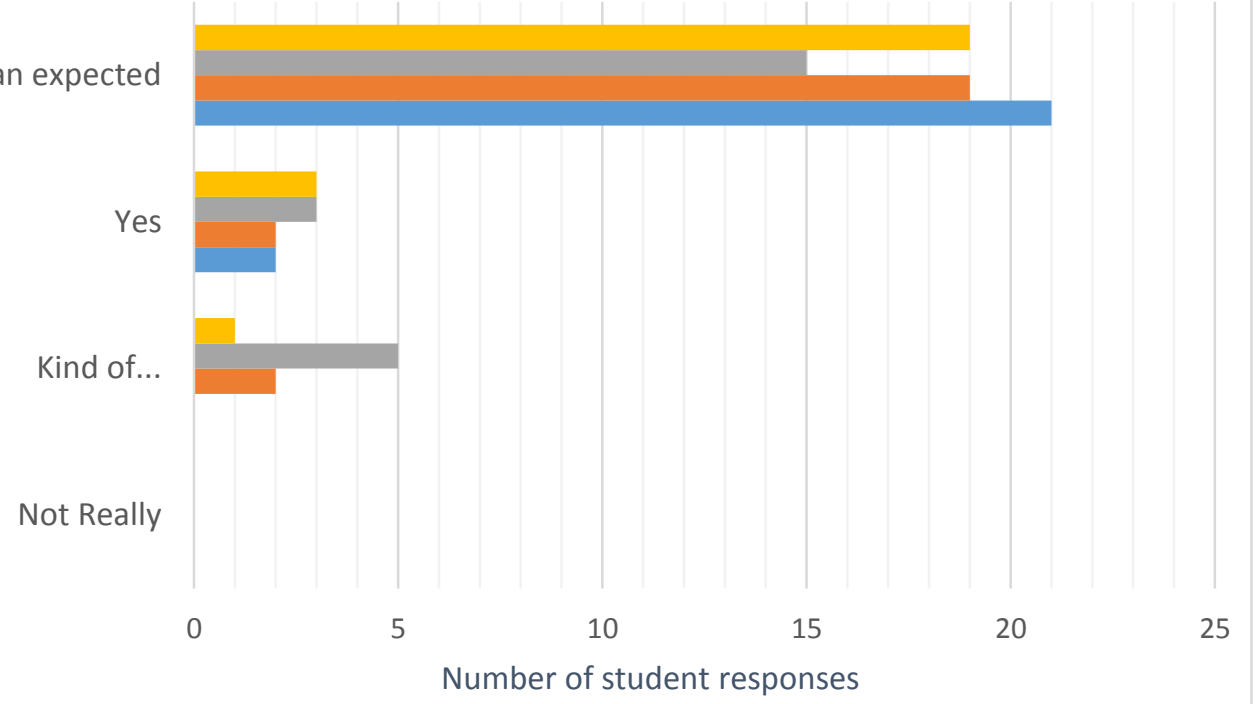

\begin{abstract}
Number of student responses
Did this lab meet the stated objectives of performing a basic fault study, obtaining results for three-phase, single line-to-ground, line-to-line, and line-to-line-to-ground faults in the relevant parts of the system and interpret the results?

Did this lab meet the stated objective of identifying the results of the power flow that have an influence in the fault study?

Did this lab meet the stated objective of performing basic analysis of the results of the Power Flow function of the ASPEN software on the radial and looped systems?

Did this lab meet the stated objective of creating simple cases in ASPEN One-Liner, including positive-, negative- and zero-sequence impedances of line and generators as well as proper transformer connections?
\end{abstract}

Figure 33: ASPEN Introduction Survey Results

\title{
5.2.1.2 Laboratory 1 Student Comments
}

Comments referring to the overall lab were, in general, positive and included statements such as "Very good introduction to ASPEN" and "The fact that the lab is divided into multiple groups is a really plus to the lab, really great TA, and a good learning experience all in all." One student states that all requirements were met, where "requirements" is interpreted as the stated objectives for the lab and the feedback is interpreted as positive. Negative comments included students requesting more model detail in the written 
instructions and how to read the software results table referred to as the TTY. One student requested a short presentation on how to use ASPEN, a task that was not feasible due to technological, physical space, and time restrictions. Rather, TAs were actively providing individual assistance to groups during model building, simulation, and data analysis. One student suggested a simpler model for this assignment as an improvement but did not elaborate as to why. While this information is taken into account, the models in this assignment were made as simple as possible in order to accommodate for time restrictions and to introduce the radial and looped system configurations.

Student willingness to explore and learn with the given materials were indicated in comments mentioning having to "figure some things out in ASPEN but was good learning experience as an engineer." and "After some effort, the TTL output could be easily interpreted." These comments were considered to be positive in nature because they show both a willingness, and an ability, by the students to use the tools provided to expand their own learning while also directly meeting the overarching experimentation and data analysis outcomes of this teaching laboratory.

\subsubsection{Laboratory 2 - Thermal Properties Survey Results}

The thermal properties laboratory survey had twenty responders assessing the

effectiveness of the five objectives listed at the beginning of the laboratory instructions. The survey was given in the same multiple choice format as the previous survey, 
allowing for short answer comments at the end of the survey to enhance the analysis of the multiple choice section. The five objectives of the laboratory were,

- Did this lab meet the stated objective of building the temperature-vs-time heating curve of a wire in free air?

- Did this lab meet the stated objective of requiring students to compare the results of the experimental $\mathrm{T}(\mathrm{t})$ curve with the predicted results and the equation from lecture notes?

- Did this lab meet the stated objective of experimentally building an approximate time-current, I(t), curve for the fuse element?

- Did this lab meet the stated objective of verifying fuse element material type based on thermal behavior?

- Did this lab meet the stated objective of assisting the student to be able to explain the application of a time-current static curve and the dynamic temperature-time response of electrical equipment?

\subsubsection{Results of Laboratory 2 Assignment Objectives}

For the first objective of the assignment regarding construction of a Temperature v. Time heating curve of a wire in free air, fourteen of the twenty survey participants recorded "Yes" and four students responded "Yes and then some". Only two of twenty students responded "Kind of..." and zero students responded "Not really". 
The objective requiring student to compare the results of the experimental $\mathrm{T}(\mathrm{t})$ curve to the theoretical results calculated had thirteen response of "Yes" and four responses of "Yes and then some". There were three responses for "Kind of..."and zero responses for "Not really". The majority of responses for this objective indicated that the students participating in the survey found this objective to be met or exceeded.

The third objective regarding experimentally building an approximate time-current, $\mathrm{I}(\mathrm{t})$, curve for the fuse element received a very high positive response of "Yes" for seventeen of the twenty responders and the final three survey participants recorded "Learned more than expected". There were zero responses for "Kind of..." and "Not really". This was another overwhelming positive response of an objective being met for an assignment. Assessing the objective of verifying fuse element material type based on thermal behavior found that sixteen of the twenty responded "Yes" with three responses of "Learned more than expected" while one responder recorded "Kind of..."with regard to this objective being met. There were zero responses for "Not Really".

Survey participants found that the final objective of the assignment effectiveness in assisting students to be able to explain the application of a time-current static curve and the dynamic temperature-time response of electrical equipment was successful. For this objective twelve of twenty students responded "Yes" to the objective being met while four students responded "Yes and then some" and four students responded "Kind of..." There were zero response of "Not Really". 


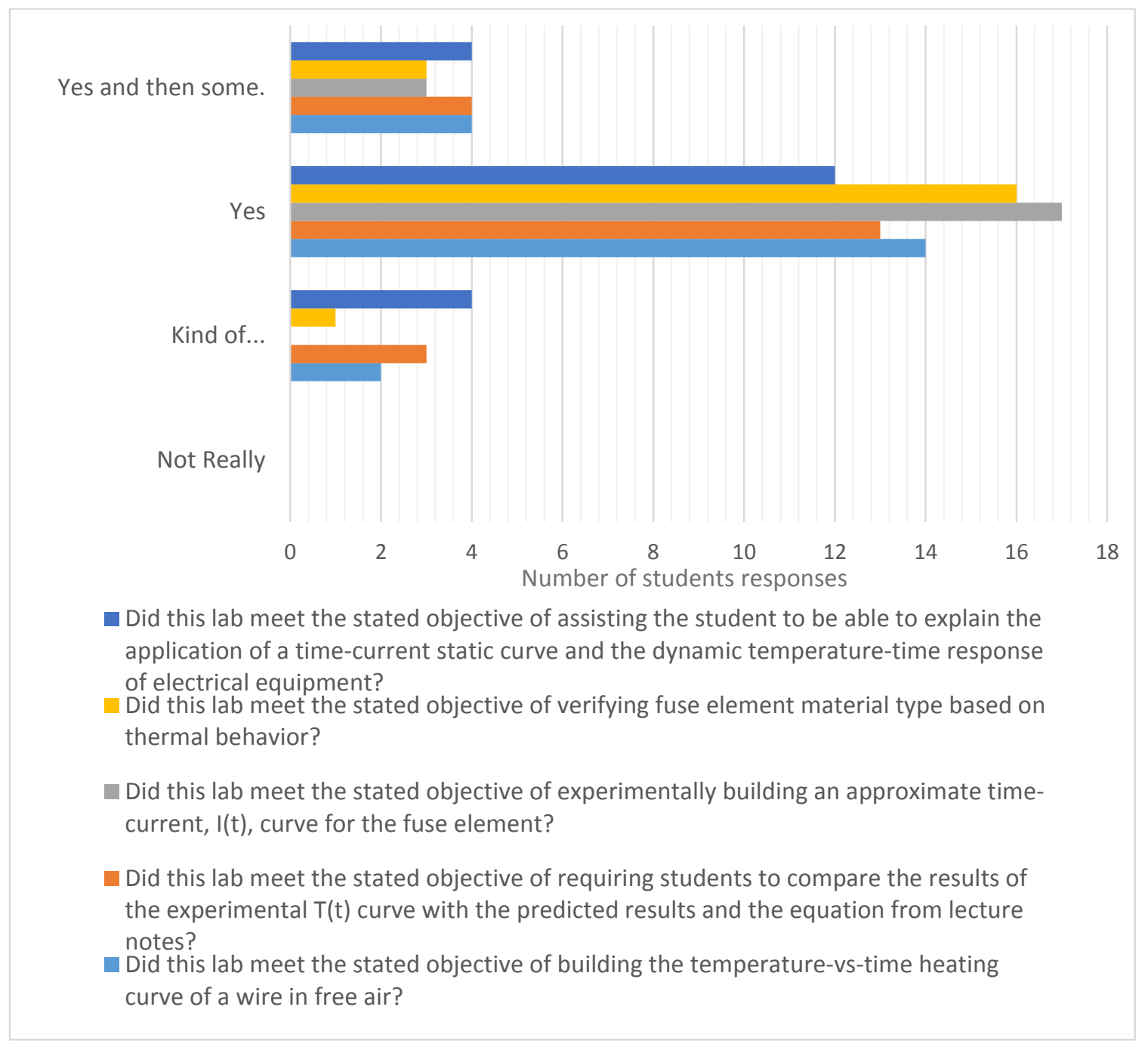

Figure 34: Thermal Properties Survey Results

\subsubsection{Laboratory 2 Student Comments}

Of the twenty responders, eleven left comments. All comments were considered positive, with one student critique of the equipment malfunctions creating issues with the execution of the lab and effected the accuracy of the experimental data, "This lab was interesting in the fact that the damage temps/curves were physically measured. "The frustrating part was that the SR51 supply unit did not work correctly which wasted our 
time. We did not get the correct time to current measurements due to this fact." One response was considered erroneous due to its nonsensical statement to, "please wrap-text Q\#4 for easier reading.” As the lab instructions for this lab assignment do not contain any numbered questions or any spreadsheet cells, it is assumed that this comment was intended for a different assignment. The other comments iterated student enjoyment in the assignment while also finding the laboratory useful in reinforcing lecture subject material. Sample comments regarding the laboratory success include, "This lab work was helpful" and "This lab was fun as well as informative."

\subsubsection{Laboratory 3 - Auxiliary Relay Testing Survey Results}

The third assignment of the laboratory introduced students to the mechanics of an electromechanical relays and testing of instantaneous OC settings on the auxiliary relays. The auxiliary relay testing lab assignment was the shortest and simplest laboratory assignment, with only three objectives outlined,

- Did this lab meet the stated objective of identifying the most relevant parts of the relay testing equipment?

- Did this lab meet the stated objective of testing the pick-up value and operation times of the electromechanical auxiliary relays?

- Did this lab meet the stated objective of familiarizing you with the features and capabilities of the electromechanical relay test equipment?

\subsubsection{Results of Laboratory 3 Assignment Objectives}


For this survey, there were twenty-three responders. The first laboratory objective to identify the most relevant parts of the relay testing equipment had twelve of the students answer "Yes" for this objective and thirteen recorded "Learned more than expected". Only one responder, replied "Kind of..." There were zero responses of "Not really" for this objective.

The objective of testing relay pick-up value and operation times had a high positive response with seventeen of the twenty-three students responding "Yes", seven responding "Yes and then some", and two responding "Kind of...". There were zero responses of "Not really."

Twenty of the twenty-three survey responders left comments regarding their experience with the laboratory assignment. Overall the students found the objective of familiarizing students with the features and capabilities of the electromechanical relay test equipment successful. Of the twenty-three responders, sixteen recorded "Yes" for the objective having been met. Six students recorded "Yes and then some", with four of twenty-three responding "Kind of...." and there were zero responses of "Not really". 
Yes and then some
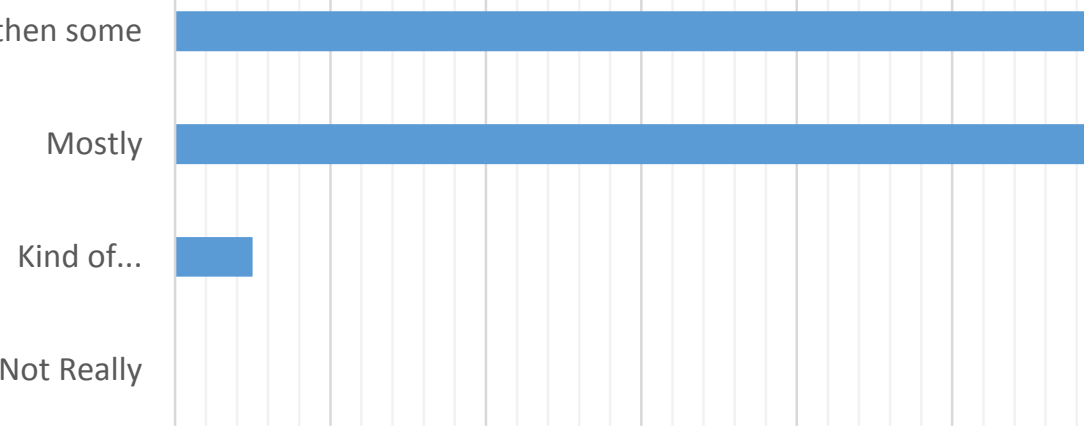

0
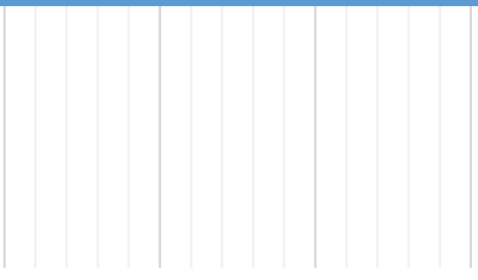

6

8

10

12

Number of student responses

Did this lab meet the stated objective of identifying the most relevant parts of the relay testing equipment?

Figure 35: Auxiliary Relay Testing Objective 1 Survey Results

Learned more than expected
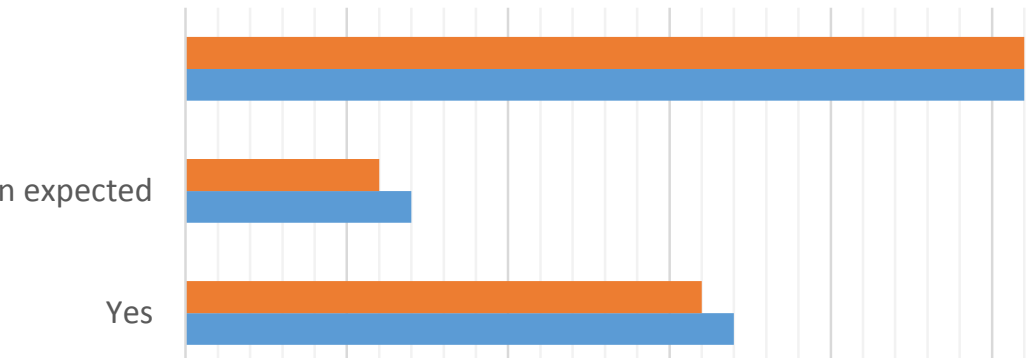

Kind of...

Not Really

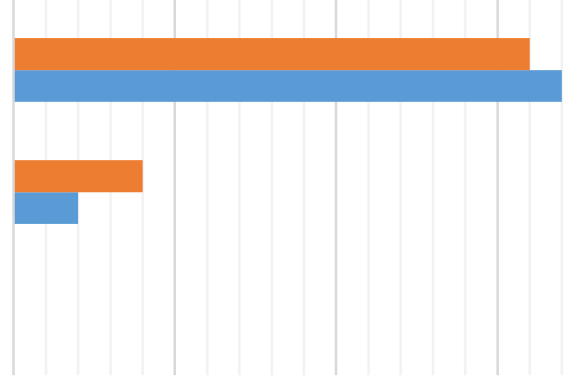

5

10

15

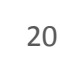

20

25

30

Did this lab meet the stated objective of familiarizing you with the features and capabilities of the the electromechanical relay test equipment?

Did this lab meet the stated objective of testing the pick-up value and operation times of the electromechnical auxiliary relays?

Figure 36: Auxiliary Relay Testing Objectives 2 \& 3 Survey Results 


\subsubsection{Laboratory 3 Student Comments}

There were a range of responses in the student comments regarding student perception of the laboratory assignment. One student wanted a more explanation of the assignment in the introduction of the laboratory, "a more in-depth explanation of lab" while another student's perspective was that there was too much instruction and not enough space for personal learning, "This one is gonna take some practice. I wish there were a way that we could have been led to 'discover' more." A couple students stated they wanted more explanation of the testing equipment knobs and relay testing manuals. The most comprehensive student feedback was given as follows, "Lab assignment: great, have the student get their hands on this equipment which is truly ubiquitous in the industry. Awesome experience! Procedures: thank you for making whatever manuals available as a resource on D2L. Some of these things were made before many of us were born, so said manuals can be hard to find (not impossible) with a mere Google search. Having that resource available saved valuable time that the student can spend interacting with the relays. TA instruction: outstanding! It's very difficult to learn something new when you don't even know what you're looking at. Framing what the relay does, what it's components are, how it might function, was a good start for the student down the path of thinking about how this device might be used in practice in the field. I assure you that even if the TA would 'spoon feed' information about every detail of the relay, there would still be questions. And that's where the learning is, in students' asking questions. Also, the test equipment was not necessarily intuitive, so having a bit of help with it saved valuable time that the student can use thinking about the relay and how 
it works, not being frustrated about how the damned testing machine works. Overall, this lab gives students a good launching point for their own discovery and independent thought about relays in general and these long-in-the-tooth, but reliable mastodons. Can we have more play time, please?!"

The majority of comments for this assignment were overwhelmingly positive and the execution of this assignment was considered successful. Several students appreciated working with the physical equipment and thought it was an entertaining assignment. One student requested more types of relays for testing, which was considered a positive response since it indicates student engagement and interest in course material.

\subsubsection{Laboratory 4 - Current Transformer Testing Survey Results}

The CT lab assignment survey had twenty responders assessing the effectiveness of the laboratory based on the four objectives outlined in the assignment instructions. The students answered four multiple choice questions regarding how well the four objectives of the laboratory assignment, as stated in the assignment instructions, were met. The short answer portion for student comments regarding the assignment was included as well. The four objectives were,

- Determine CT accuracy class and burden rating.

- Construct the magnetization curves of different CT ratios.

- Experimentally determine CT burden through magnetization curve analysis. 
- Compare the experimental results with the burden rating given by the manufacture in the CT datasheet.

\subsubsection{Results of Laboratory 4 Assignment Objectives}

For the first objective of determining $\mathrm{CT}$ accuracy class and burden rating ten of the twenty students recorded "Yes" and ten of the students recorded "Kind of..." There were zero responses of "Yes and then some" and zero responses of "Not really".

For the stated objective of the construction of the magnetization curves for the different CT ratios, magnetization curve analysis had the same response where fifteen students responded "Yes". Five students responded "Kind of...." There were zero responses of "Yes and then some" and zero responses of "Not really".

The next objective for the lab of experimentally determine CT burden through magnetization curve analysis had the same response where fifteen students responded "Yes. Five students responded "Kind of...." Again there were zero responses of "Yes and then some" and zero responses of "Not really".

The final objective of comparing the experimental results with the burden rating given by the manufacture in the CT datasheet had thirteen responses of "Yes" and seven "Kind of..." There were zero responses of "Yes and then some" and zero responses of "Not really". Indicating that this objective was not exceeded by the assignment but that the assignment needed improvements to fully meet this objective. 
It is notable that for this assignment zero students recorded "Yes and then some" for any of the objectives along with zero responses of "Not really" for all the objectives. The lack of responses for "Not really" were especially intriguing due to this particular assignment running into various equipment problems.

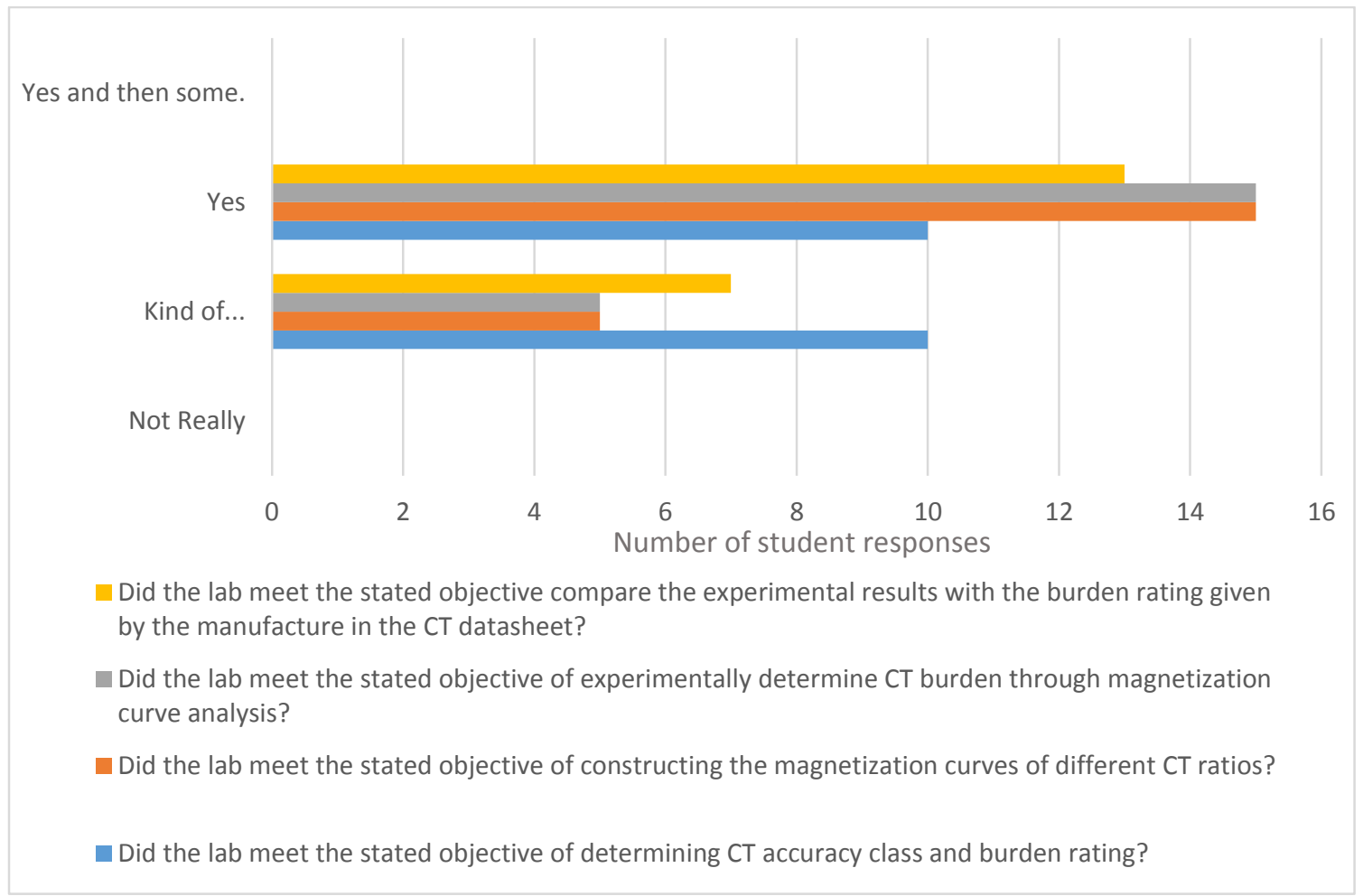

Figure 37: Current Transformer Testing Survey Results

\subsubsection{Laboratory 4 Student Comments}

The majority of the comments addressing student experience with the assignment referenced the issues with the $\mathrm{CT}$ retaining saturation after their first saturation test. Sample comments of these issues were, "CT used was highly magnetized during all experiments, hence the magnetization curve obtained was not a good reflection of its accuracy and range. Having additional CT's to use for a 'sacrificial' experiment and then 
collect useful data with would be an improvement.", "The correct or expected CT curves did not really occur on our 2 units. Since the CT manufacturer does not have any curves on their website data sheet, it was difficult to analyze and figure out what went wrong.", and "CT's a bit difficult to calibrate (keeps staying at saturation)".

There were two responses which were difficult to interpret for the assignment. One student stated it was "ironic using a more accurate CT to measure the intended CT", which might relate to the oscilloscope probes used to record the CT behavior. Another student reported, "[w]e learned about relays more" which was interesting since the assignment did not directly address relays.

Two of the student commenters left positive, but vague feedback regarding the CT assignment. One student found the laboratory assignment "helpful", but did not detail the reasons for what specifically was helpful about the lab. This same student did request the Tektronix software to be installed on the laboratory desktops to aid in data collection during the experiment. The other positive student comment on the lab referenced the student's appreciation of having hands on experience with the CTs.

\subsubsection{Laboratory 5 - Electromechanical OC Testing Survey Results}

For the fifth laboratory assignment covering EM OC relay operation principles, nineteen students participated in assignment feedback. This assignment dealt with the physical inspection and testing of General Electric IAC relays donated from PGE. The quiz followed the same format of a multiple choice question addressing each of the 
assignment objectives along with a short answer portion for student comments regarding their experience with the assignment. The five objectives specific to this assignment were,

- Identify the most important parts of an electromechanical over-current relay.

- Explaining the application of each part of the relay.

- Determine the pick-up current of the inverse-time relay element using a relay testing equipment.

- Determining the instantaneous current of the inverse-time relay element using relay testing equipment.

- Compare the results of the relay operation time with the time curve characteristics given by the manufacturer in the relay manual.

\subsubsection{Results of Laboratory 5 Assignment Objectives}

For the first objective of identifying the most important parts of an electromechanical over-current relay fifteen of the nineteen responders recorded "Yes" and three students recorded "Yes and then some", giving this particular objective a 95\% positive report of being achieved. There was one response of "Kind of..." and zero responses of "Not really".

For the second objective of determining the pick-up current of the inverse-time relay element using a relay testing equipment sixteen of the students recorded "Yes" and three students responded "Yes and then some". There were zero responses of "Kind of..."and 
"Not really", indicating that this objective was either fully met or exceeded based on the experience of all the students responders for this assignment.

Sixteen of the nineteen responders recorded "Yes" for the third objective of determining the pick-up current of the inverse-time relay element using the relay test equipment. Two of the responders recorded the laboratory exceeded in meeting this objective by recording "Yes and then some". There was one student response of "Kind of..."with regard to experiencing and zero responses of "Not really".

For the fourth objective of determining the instantaneous current of the inverse-time relay element using the relay test equipment, fifteen of the nineteen students responded "Yes" and two students responded "Yes and then some". There were two responses of "Kind of..." and zero responses of "Not really".

The final objective for the assignment comparing the results of the relay operation time with the time curve characteristic given by the manufacturer in the relay manual had fifteen student responses of "Yes", two responses of "Yes and then some". For this objective there was one response of "Kind of..."and one response of "Not really". 


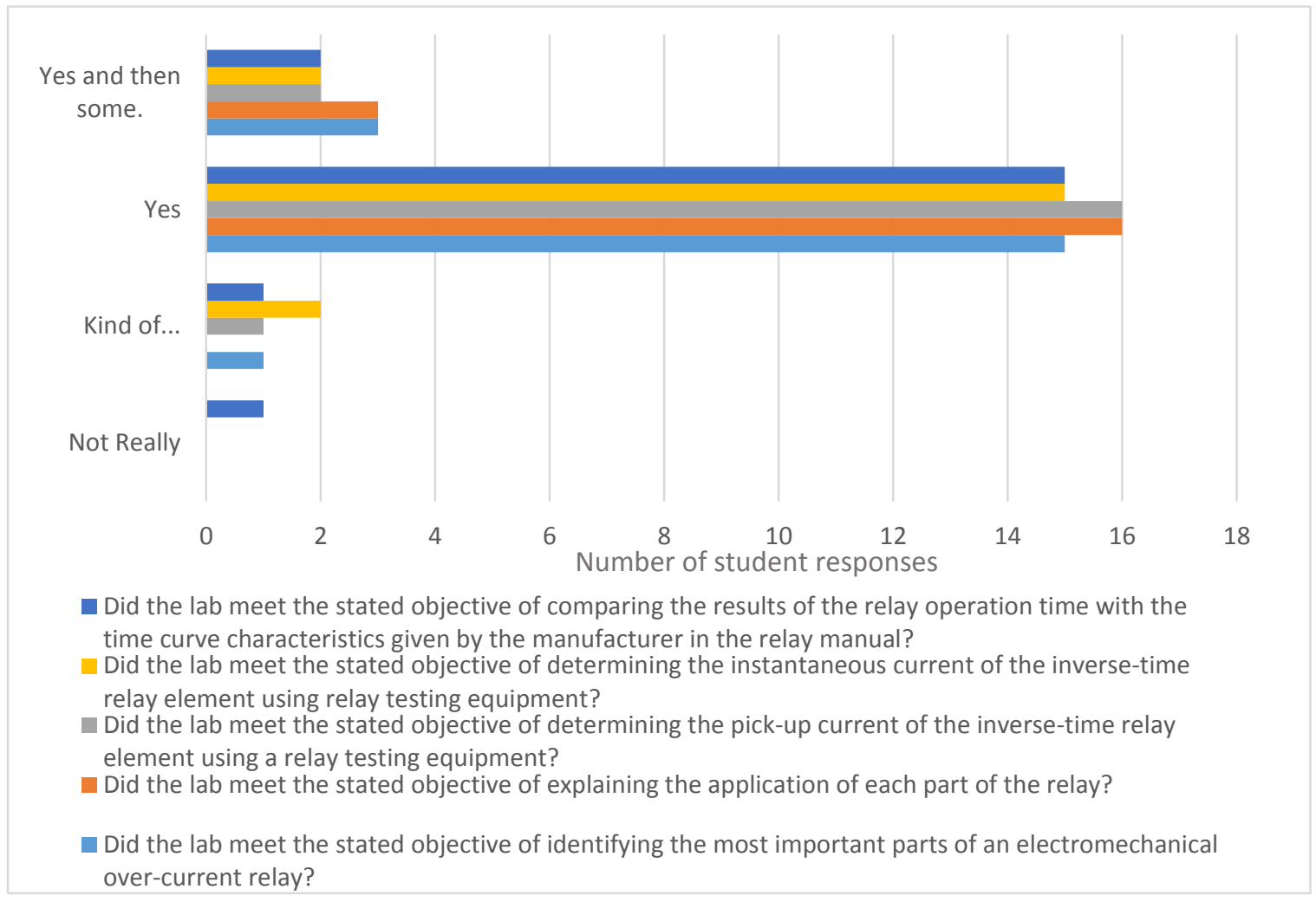

Figure 38: EM Relay Radial Over-current Protection Survey Results

\subsubsection{Laboratory 5 Student Comments}

There were ten students who left comments regarding their experience with the assignment, the majority of which were positive. Overall the comments were positive and indicated that the students enjoyed the assignment and found it useful. Sample comments of "I really enjoyed this lab because actually creating the curves made what the curves actually represent make more sense" and "Great working with everyone. Happy to be practicing real relay analysis." One student commented "Very clear on what will I learn in the lab." which was interpreted that the student experienced the objectives 
to be successful as outlined in the written instructions of the assignment. One student commented that the assignment was interesting.

The only negative feedback from the comments referenced issues with testing the relay instantaneous element, "determining the instantaneous current was a bit difficult with the type and age of relay we were using". Another student requested further explanation using the software and mentioned the difficulties of using ASPEN, indicating that they were responding to a different laboratory assignment, since no software was used in this assignment. One student requested more information for coordination design, which was addressed in the following laboratory assignment and was purposefully omitted from this assignment.

\subsubsection{Laboratory 6 - ASPEN Radial OC Coordination Survey Results}

The sixth laboratory assignment had eighteen responders reviewing the effectiveness of the lab objectives based on their experience with designing OC protection for a simple radial system. The same radial case used in the ASPEN introduction assignment was used for this assignment, with the addition of relay protective equipment added to the system circuit breakers. The four objectives specific to this assignment were,

- Did the lab meet the stated objective of creating a radial case in ASPEN to do fault studies and overcurrent relay coordination?

- Did the lab meet the stated objective of introducing data of phase and ground overcurrent relay elements into the model? 
- Did this lab meet the stated objective of simulating faults and determining the behavior (time response) of the overcurrent relay elements?

- Did this lab meet the stated objective of performing a coordination study to determine relay settings?

\subsubsection{Results of Laboratory 6 Assignment Objectives}

For the first objective regarding the creation of a radial case in ASPEN to perform fault studies and OC protection, sixteen responders recorded "Yes" for the objective being met and two responders recorded "Yes and then some". There were zero responses for both “Kind of..."and "Not really". These results gave a unanimously positive response from the eighteen participants of this assignment survey.

The second objective was related to introducing data of phase and ground elements into the model. Fifteen of the eighteen students responded "Yes" while responded "Yes and then some". There was one response of "Kind of..."to this objective and there were zero responses of "Not really".

The third objective regarding fault simulation and determining OC relay element behavior had fourteen of the students respond "Yes" and three students recorded "Yes and then some". There was one student who found this objective not to have been fully met and responded "Kind of..." and there were zero responses of "Not really". 
Regarding the final objective of performing a coordination study to determine relay settings thirteen responded "Yes" while five of the eighteen student responders reported "Yes and then some" for this objective having been met. There were zero responses for "Kind of..."and "Not really". These results gave a unanimous consensus among the survey responders that the assignment objective for performing coordination studies as part of radial OC coordination had either been met or exceeded, based on their personal experience performing the assignment.

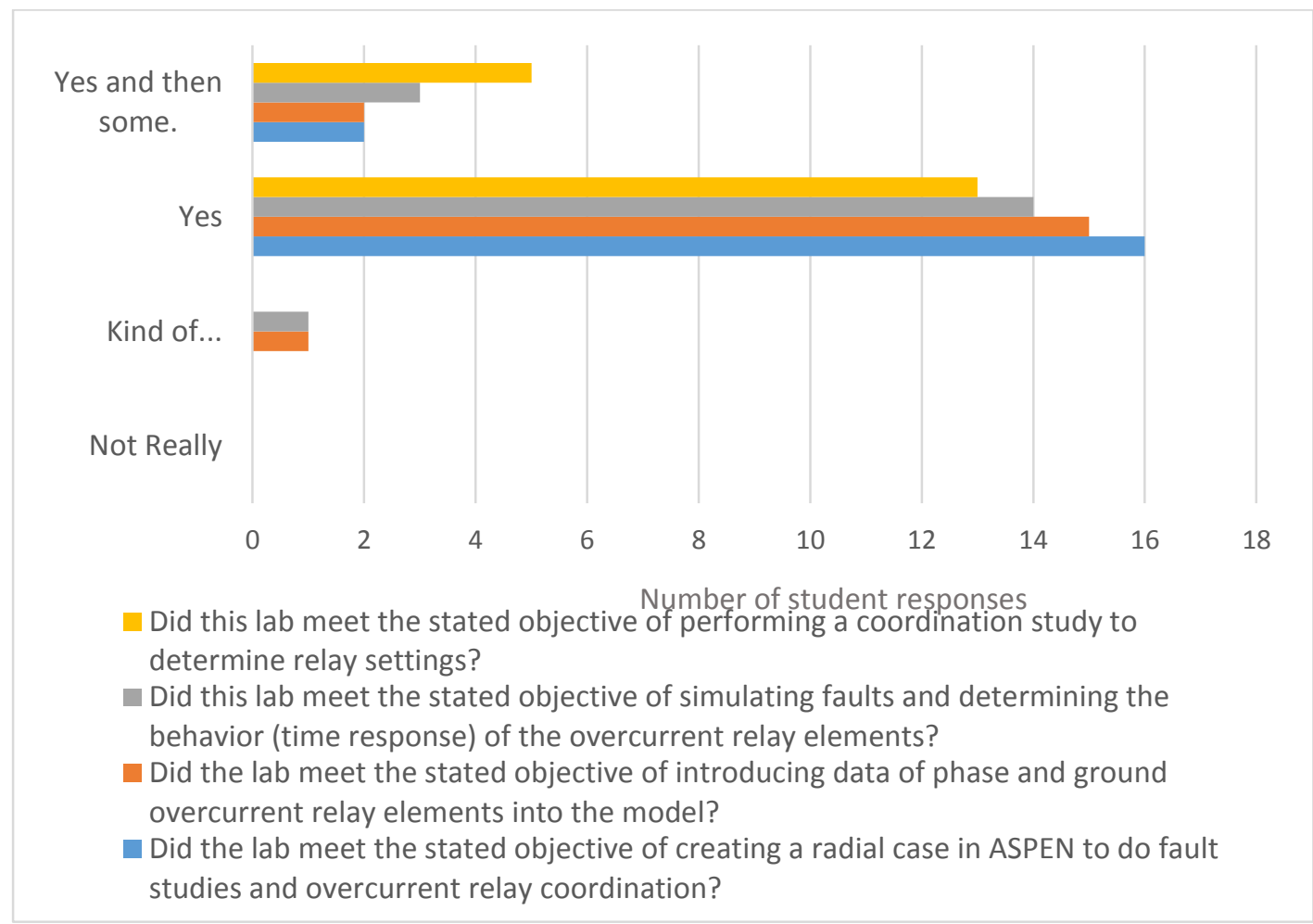

Figure 39: ASPEN Coordination of OC Relays in Radial Systems Survey Results

\subsubsection{Laboratory 6 Student Comments}

There were nine comments left by the eighteen responders for this assignment. Overall the laboratory was considered successful due to the practical experience of using industry 
standard software. Sample comments include "It was nice to see the theoretical relay values matched the physical values for proof of concept." and "Very helpful to see how a protection engineer might do 'coordination'."

The learning curve of using the ASPEN software was addressed in the comment, "introducing data of phase and ground overcurrent relay elements into the model was somewhat confusing". One responder stated "It would be nice to see how easy the other program works (forgot the name) compared to aspen." Again, one student repeated their request for further information covering "good coordination design". One student wanted more time on this assignment but did not give a reason for this. Only one student addressed the issue of the assignment delay behind the lecture material, which was due to the addition of the individual CT assignment, and therefore kept students from being exposed to practical applications of radial coordination until after the midterm. This commenter specifically stated, "Would be good for this lab to happen about the same time coordination is discussed in class and preferably before it is tested in a mid-term exam.”

\subsubsection{Laboratory 7 - Digital OC Relay Testing Survey Results}

The seventh laboratory assignment covered programming and testing the digital SEL OC relays had sixteen responders. This assignment involved verifying test wiring connections, settings input, and interfacing the relay equipment with the PC. This 
assignment had sixteen responders who answered six multiple choice questions regarding each of the assignment objectives. The six objectives specific to this assignment were,

- Implement physical set-up to test SEL-551 using the SEL-RTS (AMS).

- Communicate with the relay using the SEL-AMS.

- Introduce settings to the relay (Phase and ground elements).

- Properly set a test using the SEL-5410 software.

- Test the relay with the AMS and obtain operation times.

- Verify operation results by comparing with results found under software simulation.

\subsubsection{Results of Laboratory 7 Assignment Objectives}

Regarding the first objective on whether the assignment was successful in implementing a physical test setup and programming of settings to the digital relays, thirteen of sixteen students responded "Yes" and two students responded "Yes and then some" with regard to the first objective being met. One student responded "Kind of..."while zero students responded "Not really".

Similarly to the first objective, the objective reviewing the effectiveness of the assignment leading students to setting a relay test case in the SEL vendor software had thirteen student responses of "Yes" and "Yes and then some". One participant responded "Kind of..." while zero students responded "Not really". 
For the objective regarding the establishment of relay-to-test equipment communication, also known as a "handshake", thirteen of students recorded "Yes" and three students recorded "Yes and then some", while zero students responded "Kind of..." or "Not really". All survey participants considered this objective either met or exceeded. Of the sixteen student responders, twelve participants recorded "Yes" for the objective of successfully testing the relay with the SEL testing software. Three responded "Yes and then some", and one student responded "Kind of..." There were zero responses of "Not really".

The final objective for the digital OC testing assignment, regarding the verification of the tested conditions of the relay with the simulated results from ASPEN in the previous assignment, saw thirteen responses of "Yes" and one response "Yes and then some". Two participants recorded "Kind of..." and zero students recorded "Not really". The majority of participants considered this objective either met or exceeded. 


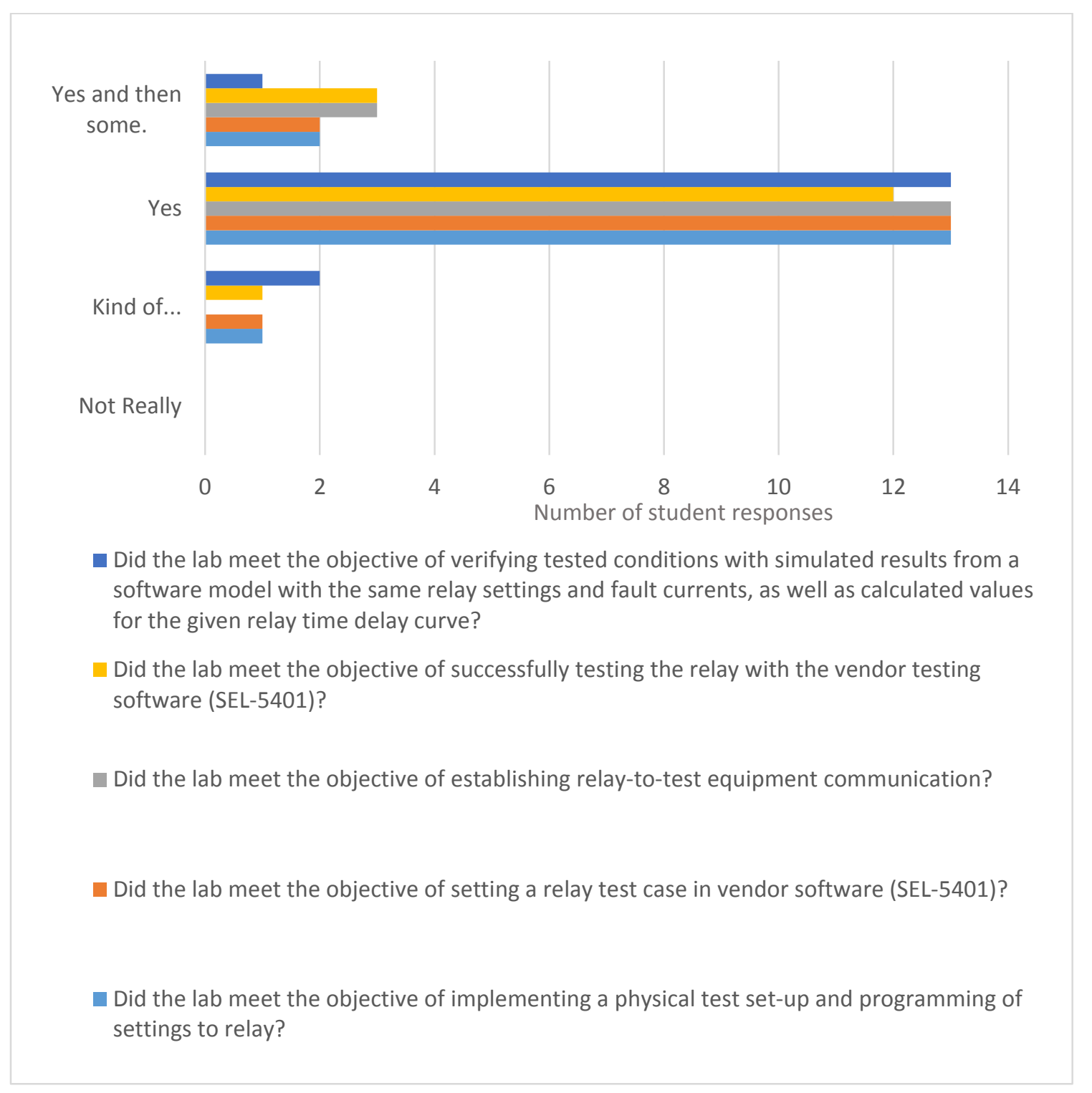

Figure 40: Digital Relay Radial Over-current Protection Survey Results

\subsubsection{Laboratory 7 Student Comments}

Nine students left comments regarding their experience with the assignment. One student gave an appropriate response for the subject material of radial coordination, "This one took a while to sink in". One student referenced working with the "new" relay tester and trying to find the "10-20 connections" of the relay, which refers to the digital and 
mechanical relays in the ninth laboratory assignment involving the SEL-351 relays and the EM JBC51N relays. One student responded "Next year's lab will be great", but the student did not elaborate as to why the current lab was not considered 'great'.

Otherwise, student comments were positive. Sample comments include "good to know that SEL can accurately provide outputs" and "Great hands-on experience". There was nothing in the comments suggesting direct improvements to the assignment nor were there comments that directly related specifically to the success of the assignment.

\subsubsection{Laboratory 8 - ASPEN Looped OC Coordination Survey Results}

The eighth laboratory assignment covering looped system coordination design in the ASPEN software had sixteen responders. There were four objectives related to the system model construction and protection design. These four objectives were,

- Create a looped power system case in ASPEN OneLiner containing the required information to perform fault studies and overcurrent relay coordination.

- Set phase and ground relays into the system model.

- Simulate faults and determining the behavior (operating time) of the relays.

- Performing a coordination study to determine the relay settings.

\subsubsection{Results of Laboratory 8 Assignment Objectives}

There were sixteen student responses reviewing Lab 8 assignment objectives. For the first objective of creating a looped power system case in ASPEN containing all required information to perform fault studies, twelve of the students recorded "Yes" and two 
students recorded "Yes and then some". This gave a positive response of $87 \%$ from the sixteen survey responders with regard to the first objective of creating a looped power system capable of performing fault studies and relay coordination. Two students responded "Kind of..." There were zero responses of "Not really".

Students gave the same responses for the second objective of setting phase and ground relays into the looped system model as they did for the first objective. Twelve of the participants recorded "Yes" and two students recorded "Yes and then some". Two students responded "Kind of..." There were zero responses of "Not really".

For the third objective of simulating faults and determining the relay behavior from fault results saw student participant responses the same as for the first two objectives. Twelve of the students recorded "Yes" and two students recorded "Yes and then some". This gave a positive response of $87 \%$ from the sixteen survey responders with regard to the first objective of creating a looped power system capable of performing fault studies and relay coordination. Two students responded "Kind of..." There were zero responses of "Not really".

The final objective of whether the assignment was successful in performing a coordination study to determine the relay settings found that thirteen of the sixteen student participants recorded "Yes" while two responded "Yes and then some". There was one student response of "Kind of..." Zero students responded "Not really". 


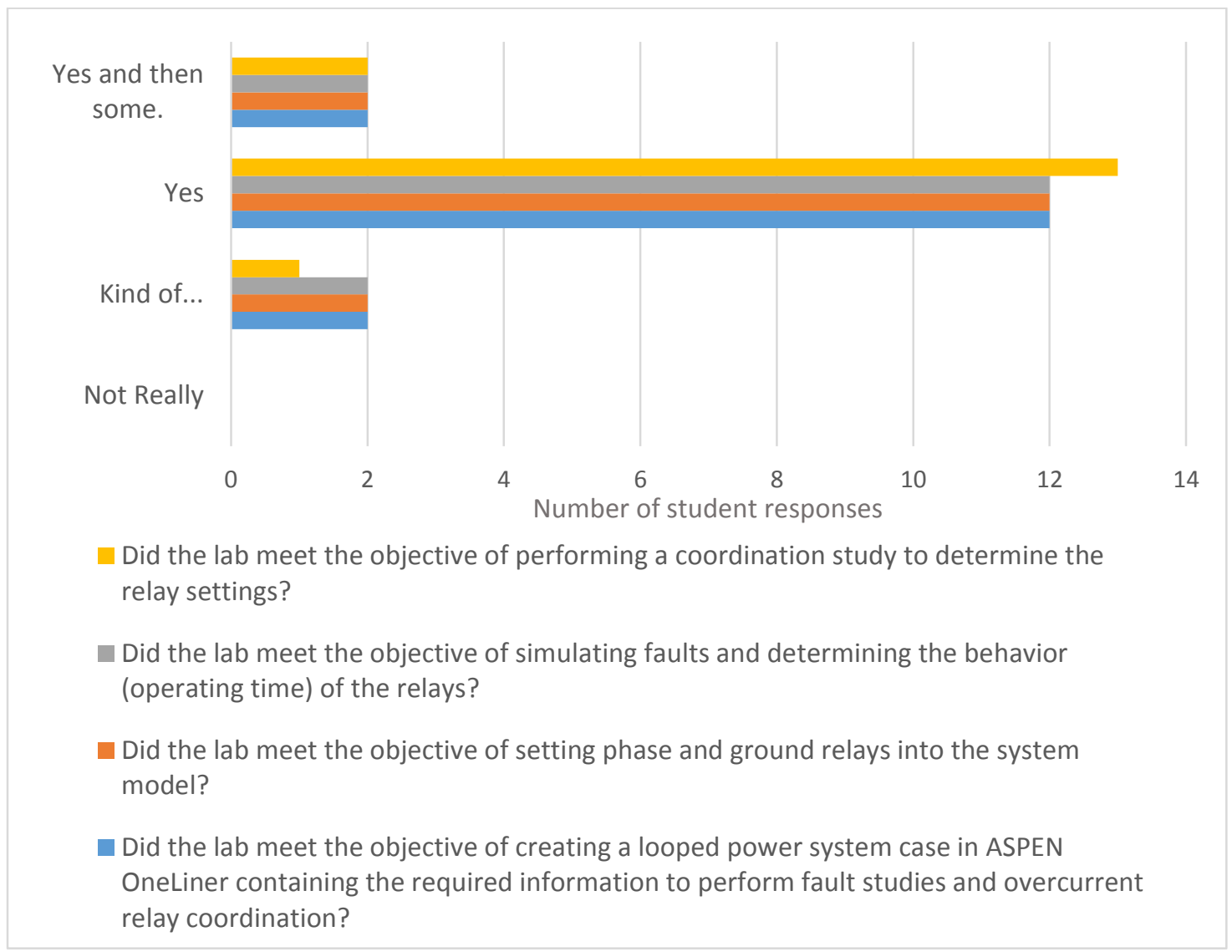

Figure 41: ASPEN Looped OC Coordination Survey Results

\subsubsection{Laboratory 8 Student Comments}

Ten participants gave feedback regarding their experience in the lab. Overall the laboratory was positively received with direct comments regarding the effectiveness of the assignment. The most comprehensive student comment detailed the assignment strength in supporting lecture material and promoting student teamwork and communication. Another student stated they appreciated this assignment building on previous laboratory assignments. One student appreciated having access to the looped system one-line file distributed by the TAs because it allowed the student's group to 
"concentrate on the lab instead of trying to backtrack and figure out what was wrong with our original one liner."

Negative comments critiqued the software, "limited to the capabilities of aspen would be nice to have another program that can do the same a little better." Another team stated issues with coordination, "Our team had problem coordinating the looped system. One relay closest to the bus fault didn't see the fault." There were two neutral comments of "N/A" and "Nothing to add" left in this short answer portion as well.

\subsubsection{Analysis of Laboratory 9 - Directional Over-current Relays Survey Results}

The ninth and final laboratory assignment for this course involved setting and testing EM and digital distance relay equipment. There were fourteen survey participants assessing the effectiveness of the four assignment objectives,

- Identify the different parts of an electromechanical directional over-current relay.

- Implement a physical set-up to test SEL-351 using the SEL-RTS (AMS).

- Communicate with the relay using SEL-RTS.

- Program the settings to the [SEL-351] relay (Phase and Neutral Ground elements).

\subsubsection{Results of Laboratory 9 Assignment Objectives}

Of the fourteen survey responders, eight of the students recorded "Yes" and four students recorded "Yes and then some" regarding the effectiveness of the first objective to the lab 
identifying the different parts of the relay. One student responded "Kind of..." and one student responded "Not really".

For the second objective, implementing a physical setup to test the SEL-351 relay using the SEL relay testing unit, twelve survey participants responded "Yes" and one participant recorded "Yes and then some". There was also one student responder who recorded "Kind of..."with regard to this objective having been met while zero students responded "Not really".

Thirteen of fourteen students responded "Yes" for meeting the laboratory objective of communicating with the relay using the SEL relay testing system while one student responded "Yes and then some". Zero percent of student responders recorded "Kind of..."or "Not really" indicating a unanimous consensus of the survey participants that this objective had been either met or exceeded in the execution of the assignment. The final objective regarding the success of programming settings to the phase and neutral relay elements found that twelve of the fourteen responded "Yes" and two participants responded "Yes and then some". Zero responses were recorded for "Kind of..." and "Not really..."indicating a unanimous consensus of the survey participants that this objective was either met or exceeded. 


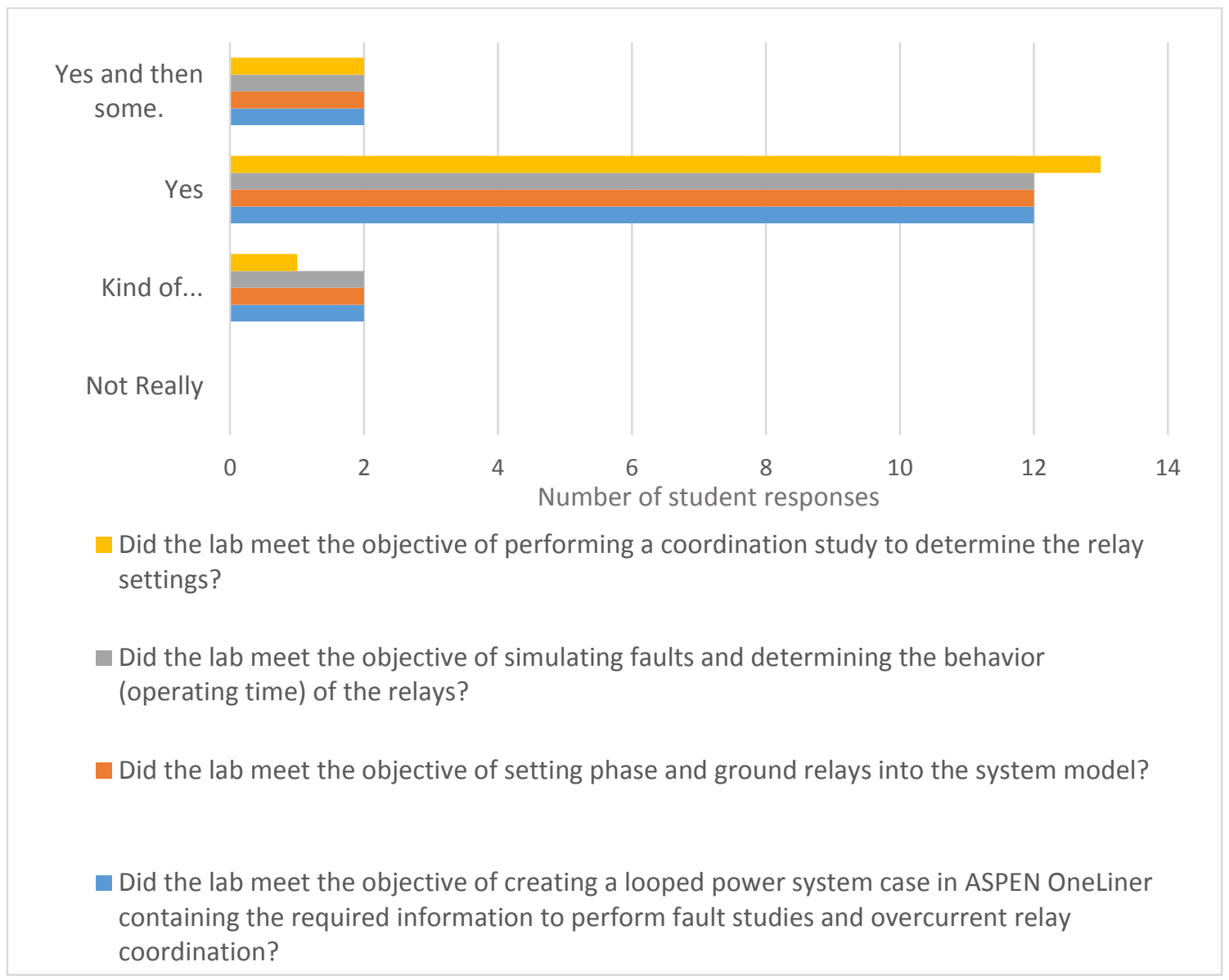

Figure 42: Directional Over-current Relays (67) Survey

\subsubsection{Laboratory 9 Student Comments}

Of the fourteen student responders, no negative comments were recorded. There was one neutral comment of "N/A". Overall student comments reflected student enjoyment of the laboratory, including in the unexpected task of dismantling a JBC51N relay to map the element contacts. Sample comments include, "taking the relay apart was extremely helpful for me as I do not have much hands-on-in-the-garage-with-my-dad experience. Also, getting tossed into the deep end with the Pulsar and only the manual was so close to 
being in a real-world type setting I though[t] I should be getting paid!" and, "Good exercise."

Overall, there were no comments detailing how the assignment could be improved however one participant expressed a desire to be able to test the JBC51N completely, since this was not possible as it was discovered that the relay instruction manual referenced control connections which were not available on the JBC51N relays in the laboratory causing a reorganization of Part I of the laboratory where students analyzed a partially dismantled JBC51N, tracing the connections to elements and comparing these connections to the wiring diagram available in the user manual to ascertain the proper connections for the particular JBC51N model in the lab."

\subsection{Overall Laboratory Outcomes Assessment Data}

At the end of the term students assessed the entire lab according to the laboratory educational objectives of the lab as outlined by Feisel. [11] [12] There were sixteen participants in the survey assessing these objectives. Each objective was evaluated in the same format as the individual assignment objectives with two short answer questions regarding making the lab a separate one credit course and overall feedback regarding the lab itself.

\subsubsection{Feisel's Laboratory Outcomes Survey Results}

There were sixteen participants for the final survey requiring student feedback for the overarching laboratory outcomes adopted from Feisel. [11] [12] 


\subsubsection{Instrumentation}

Thirteen of the sixteen participants responded "Yes" for the instrumentation outcome being met. Three of the participants recorded "Yes and then some" with zero responses of "Kind of..." and zero responses of "Not really", indicating a unanimous response from participants that this objective was either met or exceeded for the entirety of the lab exercise.

\subsubsection{Models}

Thirteen out of sixteen participants recorded "“'Yes" or "Yes and then some" for the Models outcome. Three students responded "Kind of...", and zero responses of "Not really".

\subsubsection{Experimentation}

The third outcome regarding Experimentation by providing weekly assignment descriptions clearly, articulating test procedures, experiments, and equipment for the individual labs had eleven of responders record "Yes", one student recorded "Yes and then some", and four recorded "Kind of..." Zero percent of students responded "Not really".

\subsubsection{Data Analysis}

The fourth outcome of Data Analysis through the collection, analysis, and interpretation of data collected from the testing equipment and software programs, requiring the presentation of these data through written reports and verbal discussions with lab instructors saw a ten response of "Yes", four "Yes and then some", and two recorded "Kind of..."with zero responses of "Not really". 


\subsubsection{Design}

The review of the Design outcome had intriguing result of twelve student response of "Yes" and three student responses of "Yes and then some" with one participant recording "Kind of..." and zero responses of "Not really". These results are intriguing because analysis of the result table revealed the written description of the Design outcome in the survey was repeated from Data Analysis outcome, therefore the Design outcome was not truly assessed by students. The responses were different for both outcome, even with both objective carrying the same description. A possible reason for this is that students identified more with their own interpretation of the specified outcome and relied less on the written description provided in the survey for the outcome.

\subsubsection{Learn from Failure}

The sixth outcome to Learn from Failure had the same responses as Design outcome with twelve responses of "Yes", three responses of "Yes and then some", and one recorded "Kind of..."with zero responses of "Not really". These results were surprising considering the amount of equipment failure experienced in the term, combined with the setbacks experienced in the CT lab and with the JBC51N user manual discontinuity. At least one student did not experience the ability to learn from these failures during the term, which indicates other students may not have found the lesson in failing.

\subsubsection{Psychomotor Skills}

The seventh outcome regarding Psychomotor Skills. This outcome required students to properly select, analyze, and operate laboratory equipment as well as assemble testing systems by referencing equipment data sheets, user manuals, and physically interacting 
with equipment successfully had thirteen of sixteen survey participants record "Yes".

There were two responses of "Yes and then some" and one response of "Kind of..."while zero survey participants recorded "Not really".

\subsubsection{Safety}

The eighth outcome, Safety, requiring a safety quiz regarding electrical safety prior to using the equipment saw twelve of sixteen survey participants respond with "Yes" and three participants record "Yes and then some". One student responded "Kind of..."for this outcome being met and zero survey participants responded "Not really".

\subsubsection{Communication}

The ninth overall laboratory Communication outcome accomplished through written and verbal means had fourteen of sixteen participants respond "Yes" with zero responses of "Yes and then some", indicating that while the majority of students found this outcome met, none of the survey participants found this overarching outcome of communication exceeded. Two students recorded a response of “Kind of..."and zero students reported "Not really".

\subsubsection{Teamwork}

The tenth and final the tenth overall outcome of the laboratory, assessed by how well the lab assignments promoted group work while performing lab tasks and synthesizing results into the written reports, had thirteen of sixteen responses of "Yes" and two responses of "Yes and then some". There was one survey participant who recorded "Kind of..."and zero responses of "Not really". 


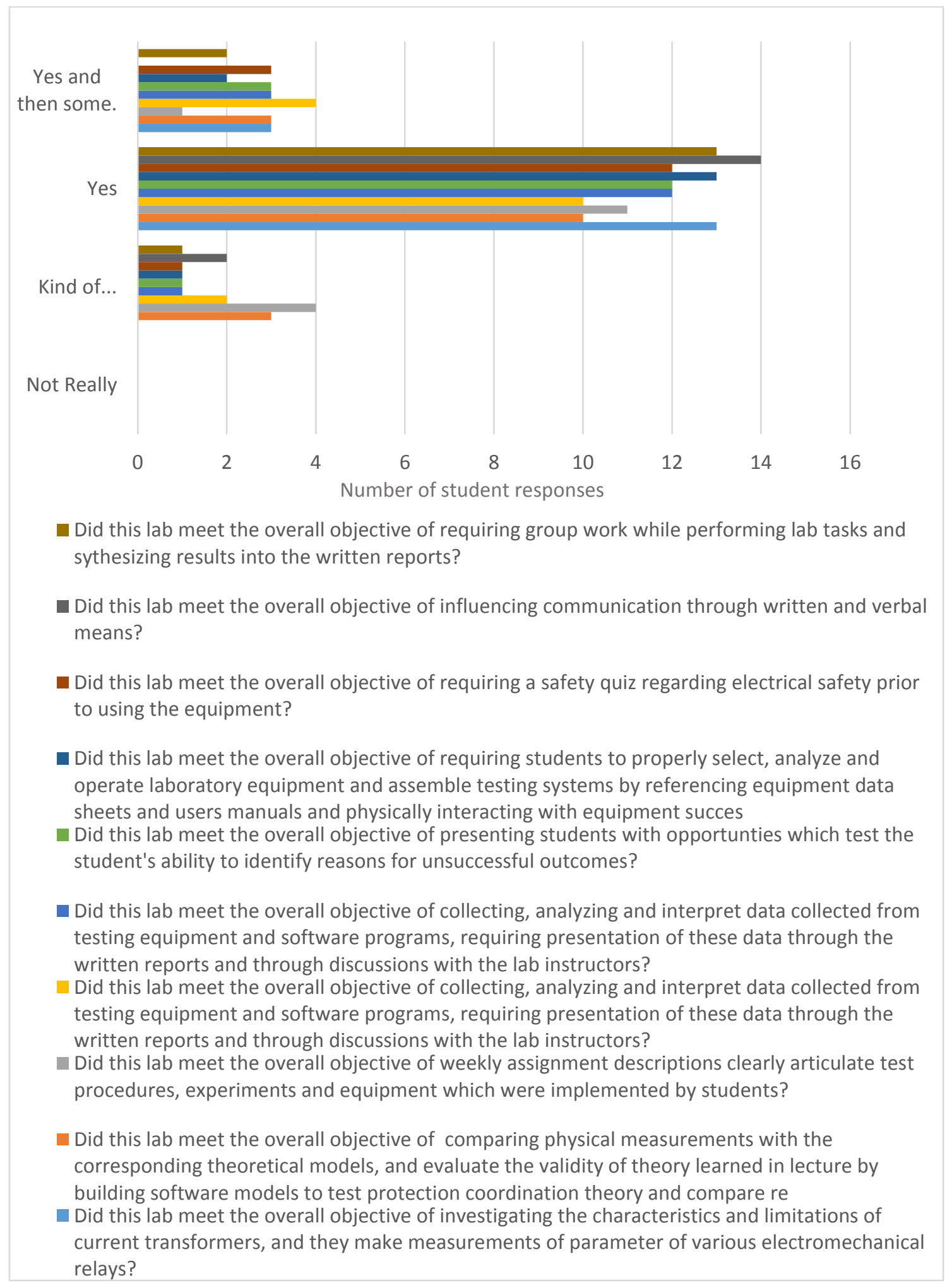

Figure 43: Feisel's Laboratory Objectives Survey Results 


\subsubsection{Laboratory as a separate, one credit course}

Ten survey participants of the sixteen replied to the short answer inquiry about whether this laboratory should be made into a one credit course. Six of these students replied with "Yes". Two students responded thought the laboratory would benefit from being a graded portion of the lecture course. One student gave an ambivalent comment of, "It makes no difference as long as one goes to school to actually learn." Sample comments include, "I think that it is important students have a typical understanding of all aspects of protection including use of common software packages. However, including this lab into the course grading may be a better option than making it a separate grade than the class. Group work does not always reflect on everyone's understanding though and presenting it separately offers a presentation of this fact in transcripts." and "I think the best thing to do would be to include the lab as a percentage of the grade of the class. An extra credit means we have to pay more." There were also a few strongly supportive responses of, "Yes, definitely!!" and "Yes! This course was helpful, but the lack of coordination between lecture and lab experiments made it a bit difficult as to 'why' we were doing some things." This last comment was an especially interesting answer for this question, since all assignment topics of the laboratory fell behind the lecture material the two times the assignment orders were changed to account for equipment needs. Students had exposure to all assignment theory from lecture before attempting the weekly laboratory assignments, indicating a gap for this student in synthesizing assignments to the lecture material presented. 


\subsubsection{Overall Laboratory Student Comments}

Of the sixteen survey participants, eleven left comments regarding the entire laboratory. In general, much gratitude and enthusiasm was shown for the laboratory, as is observable by comments such as "One of my favorite lab class from this University." and "The lab was very fun and informative." and "beneficial =)". The assignments covering relays were mentioned as being the most positively received by students. Two students felt that the lab was a lot of work and should either have less labs or be for graded credit. Two students stated further revision of the write-ups were needed along with fully functional equipment for every station. One student mentioned the faulty testing equipment as an impediment to being able to follow the assignment instructions. One student felt the assignments improved as the term went on, and expressed their gratitude for the experience.

\subsection{Rubric Results and Evaluation}

The rubrics results for the weekly assignments assess the ABET student outcomes (a), (b), (d), and (g). These were assessed on a (+), $(\checkmark),(-)$ scale, where the $(+)$ and $(\checkmark)$ were considered to positively reflect meeting the outcome criteria and the (-) was a negative reflection on the outcome criteria and illustrates where the overall program could be strengthened. For all the reports submitted, the teamwork outcome (d) was always excellently assessed at (+). There were zero $(-)$ and $(\checkmark)$ results for any of the students for this outcome, indicating that the overarching laboratory outcomes of teamwork and communication (verbally based) were met. Students also demonstrated a willingness to work group-to-group, enhancing the overall knowledge transfer of the laboratory. At no 
point during the term were there any complaints or discords regarding laboratory partners, teams, or even other classmates, which is not a standard experience in group communications and indicates a uniqueness inherent to this sample of students. Analyzed as part of the active experiment, criteria (a) regarding the understanding of engineering and mathematical principles as they applied directly to the assignment experiments was also very strong. Students were able to successfully complete the assignment objectives through the assignment experiments and some were even willing to explore inside each experiment further.

The criteria which experienced the greatest deficit in student performance were those directly assessing the quality of the written report. Consistently there was a lack of labeled axes in data plot, often figures were not labeled. For some students, the spell check function was not considered to be a valid form of personal editing. Many of the reports did not verbally discuss the outcomes of the data, failing to show the students' understanding of the engineering and mathematical principles (a) in written communication $(\mathrm{g})$. 


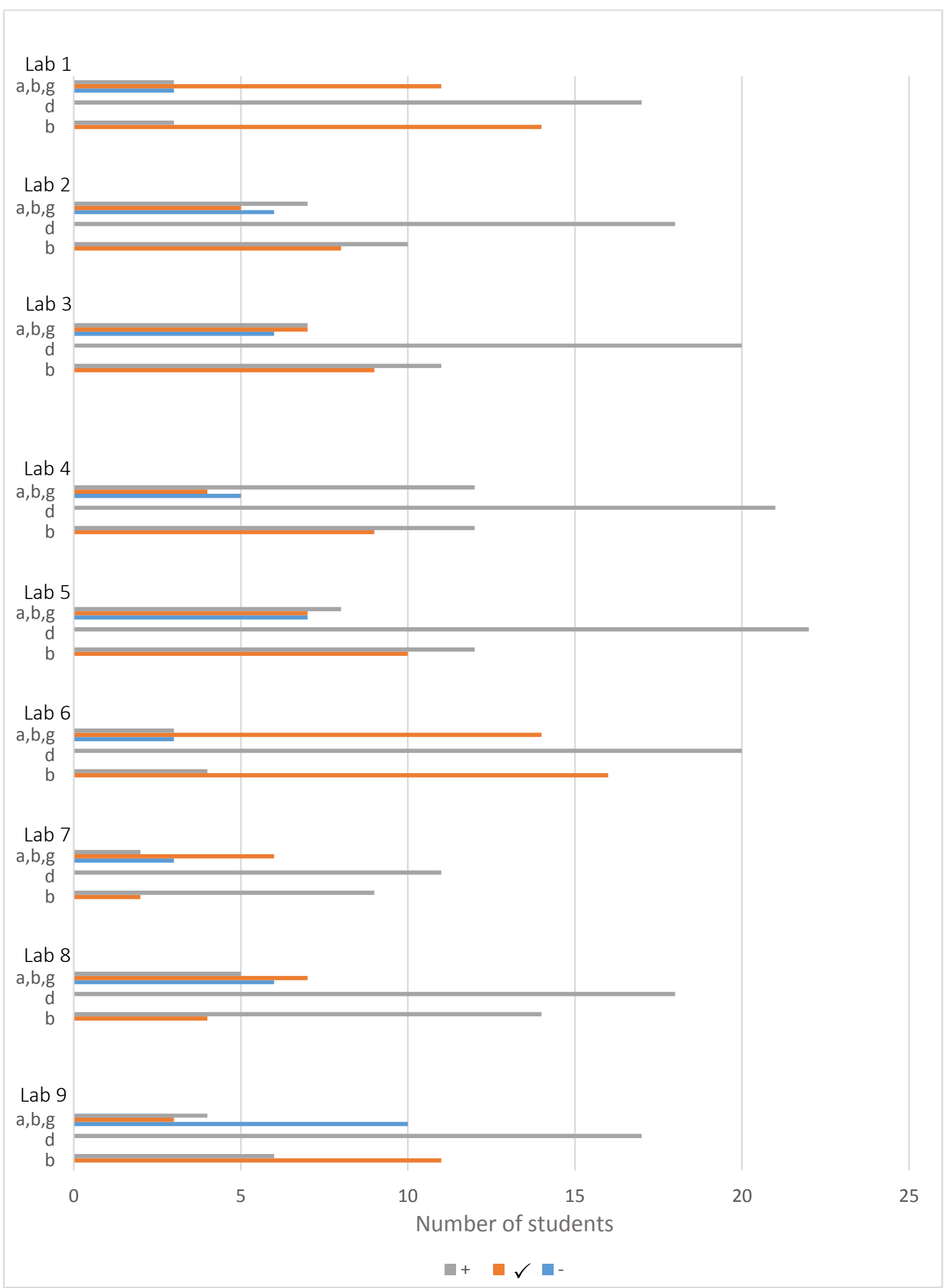

Figure 44: Grading rubric assessment data 


\subsection{Closing the Loop - Improvements to the Laboratory Based on Assessments}

\subsubsection{Survey Results for Laboratory Assignment and Feisel Outcomes}

Based on the survey assessments, each lab was edited appropriately to improve the laboratory effectiveness. The comments regarding faulty equipment which caused frustrations by impeding both the learning experience and increasing the amount of time spent on the assignments was not related intrinsically to the assignment material and therefore did not affect the editing of the laboratory assignment instructions. Appendix A lists the revised laboratory instructions to be used for teaching future terms of the laboratory, while Appendix B lists the original laboratory instructions for comparison.

\subsubsection{Laboratory 1 - ASPEN Introduction Improvements}

The majority of students gave a high level of positive response with regard to the assignment objectives. This positive feedback, combined with the student comments for the assignment, indicated only minor adjustments to this first laboratory assignment instructions were necessary. The most significant alteration to this ASPEN introduction laboratory was the addition of an appendix showing screen-shots of the parameters for each component in the looped system. This addition simplified the laboratory by providing visual aids for the inputs on the looped system. Since the students build the radial system first, and learned the method of model building and simulation the addition of this added visual aid for the looped system was not diminishing the student learning experience. This clarification directly addressed student requests for updated looped 
system model information while simplifying the execution of the assignment itself, leaving more time for simulation and analysis portions of the laboratory.

\subsubsection{Laboratory 2 - Thermal Properties Improvements}

While the overall results of this laboratory assignment were positive based on survey results for assignment objectives and student comments, the biggest improvement to this laboratory would be fully functioning testing equipment. The MultiAmp relay testers used in this assignment were decade's old, pre-owned equipment found on eBay. Based on these comments and results from the survey objectives, it was suggested that students would benefit from the addition of TTY window information in the written instructions of the laboratory assignment and in the introduction of the assignment by TAs. Since analysis of the TTY information window is part of the autodidactic nature of the assignment, no adjustments to the lab assignment instructions addressed this student concern. Future teaching terms will relay on TA discretion as to how much information is provided to read the TTY window, since TTY results are listed in a relatively straight forward format labeling the results of fault studies clearly. Since this was a senior undergraduate/graduate level laboratory course, students were expected to perform their own self-learning techniques, using tools such as internet search engines and software help menus to assist in learning how to use the software.

\subsubsection{Laboratory 3 - Auxiliary Relays}

Since this laboratory was overall successful, shown by the positive responses to the assignment objectives and survey participant comments, the instructions for the 
assignment were not altered for future teaching terms. The highly recorded success of the first objective identifying relay components is linked to the hands on introduction of the relays by the TA. Students were given their testing relays in their groups and were able to physically follow along with TA tutorial of the components and operations of the devices. It will be imperative for TAs to continue with this assignment introduction in future terms. The only alteration made to this assignment was the addition of the condensed CT testing assignment.

\subsubsection{Laboratory 4 - Current Transformers}

The CT assignment was the weakest lab, based on student surveys. No responders reported the any of the assignment objectives being overachieved. This lab also impeded students from working with the EM OC relays during the same week that the subject was taught, and consequently delayed their exposure to practical experience with OC equipment and coordination. For future terms, this lab will be included in the third assignment covering auxiliary relays in order to keep pace with the lecture material. As a result of this reorganization moving the $\mathrm{CT}$ testing to the third laboratory assignment, the following five laboratory assignments were renumbered to reflect their new teaching order in the term being a week ahead of the original curriculum.

\subsubsection{Laboratory 5 - Electromechanical Overcurrent Relays}

This laboratory was one of the most successful and popular assignments among student survey participants. Part of the success based on the laboratory objectives and student comments regarding the assignment can be attributed to the same format of introduction 
of the assignment by the TA where each lab group pulled out the OC EM relay from the mounting case and examined the relay working parts. Future terms will need to continue with this hands-on, group oriented assignment introduction. Based on student survey participant feedback of objectives and comments, no adjustments were made to the assignment instructions other than the renumbering of the assignment to reflect the teaching placement in future terms.

\subsubsection{Laboratory 6 - ASPEN Radial OC Coordination}

While the ASPEN radial OC coordination assignment was considered successful based on the survey responses to assignment objectives, several survey participant comments referenced using the digital relay equipment indicating a confusion for students who participated in the survey at later times in the term with the following laboratory assignment involving the SEL-551 digital OC relays. The comments reflected a learning curve for adding relay groups to the circuit breakers and coordinating relays according to fault studies and course lecture principles. Since this difficulty was expected due to the complexity of the subject and the novelty of the software exposure, and were difficult to fully address in written laboratory instructions, combined with the principle of the laboratory to enhance lecture course theory available in student notes and course textbook, laboratory instructions were not altered based on these comments. Assignment instructions were maintained from the original draft with the only adjustment being made addressing the change in teaching order for future laboratories due to the reorganization of the CT laboratory into the auxiliary EM relay testing assignment. 


\subsubsection{Laboratory 7 - Digital Relay (SEL-551) Testing (50/51)}

The digital OC relay testing laboratory was considered successful based on survey responses to the assignment objectives and the student comments in the survey. Verifying relay operation based on expected results from the ASPEN model was considered especially effective learning tool by the students. For this assignment, the only adjustments made to the instructions was the addition of the laboratory pre-lab exercise covering the settings of SEL-351 relay DOC elements and corresponding logic into the laboratory itself. This laboratory assignment number was also altered to reflect its teaching position in future terms due to combination of the CT assignment with the auxiliary relay assignment.

\subsubsection{Laboratory 8 - ASPEN Looped System Coordination}

The looped system coordination in ASPEN was another highly successful lab assignment, based on survey responses to assignment objectives and participant comments. As a result no changes were made to the assignment instructions other than the renumbering of the assignment to reflect its new teaching placement in future tums. The addition to the laboratory of providing a base model for students to add the relay elements to in ASPEN was highly beneficial to student focus on the looped system coordination design theory and practice adding appropriate relay elements of OC and DOC to the model. The coordination of settings, with the clockwise and counterclockwise analysis of bus fault operation for relay coordination pairs was time consuming, as was relay setting analysis and adjustment. Future laboratory courses need to provide this base model at the 
beginning of the laboratory assignment to be able to continue this focus on looped system coordination design instead of on basic ASPEN model building, which is covered in the first laboratory assignment.

\subsubsection{Laboratory 9 - EM and Digital DOR testing}

This was the only laboratory assignment to receive a response of "Not really" from one survey participant out of all of the laboratory objectives. It was discovered upon attempting to run the Laboratory tests that the user manuals available for the EM DOR relay model JBC51N (US Inverse Curve U2) had a conflicting number of input/output connections to the relay itself. Student groups used this opportunity to open the relay and start tracing connections to relay components to begin determining the proper relay connections needed to test the DOR element. No student was able to test the EM DOR element. The singular response of "Not really" regarding the effectiveness of this lab in meeting the objective of identifying the different parts of an EM DOR was likely related to this issue.

Otherwise, since this laboratory assignment was positively reviewed overall, and none of the eight survey comments suggested changes or issues with the assignment instructions. The adjustments to the labs were to detail the necessary alterations for the wiring of the input and output contacts on the relay. The relay outputs that had been labeled "1" and "11" in both the relay and the JBC instruction manuals were found to correspond with the actual physical contacts on the relay of "1" and " 10 ". The noted change was made in the 
relay tester instruction manual (Figure 45: Pulsar relay testing unit user manual instructions for

directional unit test), and included in the assignment instructions. A notes was also included with this change in the Pulsar manual to guide the students in their consideration of the DO operation during testing and how it relates to the theory from lecture material. The other adjustment made to the lab reflected the order of the assignment in its placement during the term with the reorganization of the CT assignment. This laboratory became the eighth laboratory assignment, to be performed during the ninth week during future terms.

\begin{tabular}{|c|c|c|c|c|c|c|c|}
\hline \multicolumn{7}{|c|}{ PULSAR TEST CONDITIONS } & \\
\hline \multirow[t]{2}{*}{ ASSIGN } & \multicolumn{2}{|c|}{ CONNECT } & \multirow[t]{2}{*}{ TEST } & \multicolumn{2}{|c|}{ VALUES } & \multirow[t]{2}{*}{$\mathrm{HZ}$} & \multirow{6}{*}{$\begin{array}{l}\text { For the laboratory relay } \\
\text { models of JCBS } \\
\text { connect } 10 \text { rather than } 11 \\
\text { There is no } 11 \text { th } \\
\text { connection on these } \\
\text { models. }\end{array}$} \\
\hline & \pm & $\mathrm{N}$ & & & $\angle$ & & \\
\hline $\mathrm{V}_{\mathrm{A}}$ & 7 & 8 & $\mathrm{~V}_{\mathrm{A}}$ & 115 & * & 60.0 & \\
\hline$I_{A}$ & 5 & 6 & $I_{A}$ & 2 & $0^{\circ}$ & 60.0 & \\
\hline MON & 1 & $11 \leftarrow$ & & & & & \\
\hline & & & & & & & \\
\hline
\end{tabular}

\section{DIRECTIONAL UNIT TEST}

1. Set $V_{A}$ and $I_{A}$ for directional unit polarity check based on Figure 24 of $\begin{array}{ll}\text { From previous } \\ \text { section of this }\end{array}$ Set $V_{A}$ and $I_{A}$ for directional unit polarity check based on Figure 24 of
instruction manual and table parameters below. Refer to Phase Angle Relationships Section. All current angles in JBC instruction manual are leading angles, those listed below are lagging angles.

2. Ramp current up or down to determine minimum pickup value.

3. Record the minimum pickup value. Be sure to note which ranges of current angles operate the directional unit. Include this information in your report with a synthesis of how these results compare to the theory of (67) operation based on current flow.

\begin{tabular}{|c|c|c|c|c|c|c|c|c||}
\hline P.F. Angle - Lag & $90-135$ & $135-180$ & $180-225$ & $225-270$ & $270-315$ & $315-360$ & $0-45$ & $45-90$ \\
\hline $\begin{array}{c}\text { Power Flow } \\
\text { KW IN/OUT }\end{array}$ & OUT & IN & IN & IN & IN & OUT & OUT & OUT \\
\hline \hline
\end{tabular}

4. Contacts should be closed when $\mathrm{KW}$ flow is IN and open when $\mathrm{KW}$ flow is out.

5. De-initiate $V_{A}$ and $I_{A}$; VOLTAGE, 1, CURRENT, 1, OFF, EXECUTE.

Figure 45: Pulsar relay testing unit user manual instructions for directional unit test 


\subsubsection{Rubric Results for ABET Student Learning Outcomes}

For the rubrics the majority of results were positive, showing that the outcome was met with a (+) or $(\checkmark)$ rating. The only outcome consistently in poor standing, with a (-) rating, was the data analysis and presentation criteria which directly impacts the ability to communicate the application mathematics, science, and engineering knowledge (a) and the ability to communicate effectively $(\mathrm{g})$. This was due to the quality of the reports. There was a consistent had a lack of labeled axes and often figures were left unlabeled. The spellcheck function was not utilized by all students to its fullest extent and some reports completely left out any discussion regarding results and their application to course theory.

\subsection{Evaluation - Outcomes and lessons learned}

Overall, student surveys showed that the stated goals of the laboratory, as listed previously, were met. The two most requested means of improvement were one, further lab instruction refinement and two, better functioning equipment that is more up to date. Labs instructions were altered as discussed in previous sections. Otherwise, the majority of comments from the survey participants indicated that this laboratory course was an important reinforcement mechanism for the lecture portion of the curriculum, with the most successful assignments involving the testing of the industry relay equipment and the ASPEN software.

In the future, the order of the labs will be maintained as originally planned to keep pace with the course lecture topics. During the assessment period, adjusting to equipment 
needs and arrival times caused a necessity of assignment reorganization as did the request from the course instructor to postpone the OC relay assignment material. The entire CT assignment was created to provide this space in lab assignment material and resulted in students started the electromechanical overcurrent relays the week following their homework assignments and midterm over the subject. Having access and experience with the physical components of this material earlier in the term will be more beneficial to the student learning experience, following the comments given by the students. Since the CT laboratory was condensed, with the overburden testing removed and the assignment added into the third assignment to accompany CT testing, the lab assignments will be able to stay aligned with lecture material to ensure students have physical exposure to the EM relays and radial coordination experience before the corresponding homework and midterm exam are due.

The EM and digital relay testing lab assignment results were the most unexpected, considering the problematic situation of a lack of accurate wiring diagram for the particular EM DOR model. While the students were unable to test the directional element, they were able to use working knowledge of wiring diagrams to inspect a JBC51N relay, attempting to discern the corresponding operation outputs and exploring the working elements of the relay in detail. The comments from the students indicated the educational value found in this task, and are attributed to the success of this laboratory from survey participant feedback. 
The digital relay testing of the SEL-351 introduced students to programming logic for relay output operation along with addition relay settings practice, continued from the digital OC relay testing of the SEL-551. While the relay was exposed to the same conditions found in the looped system model from the previous ASPEN, there was no DOC element operation recorded in the tests performed on the SEL-351. While no student comments addressed this issue in neither the assignment survey the overall objectives survey, the discrepancy between the simulated system of ASPEN and the experimental testing of the SEL-351 is noted since the results of the testing of the SEL551 digital OC relays corresponded to the results of the ASPEN simulations for the radial system OC coordination.

Considering the strong, positive feedback of the survey results, and with student comments affirming the educational usefulness of the laboratory itself, the laboratory course was considered successful. The overarching outcomes of the laboratory were met, according to student survey participant feedback. The minor adjustments made to individual assignments are expected to strengthen the effectiveness of the laboratory for the second teaching term of the course during the winter term of 2014.

For the ABET student outcomes $\mathrm{a}, \mathrm{b}, \mathrm{d}$, and $\mathrm{g}$ the majority of the reports were adequate to excellent for criteria (a) and (d). The evaluation of the criteria considering (a), (b), and (g) together for the written reports shows a lack in report quality. This is an issue to be addressed in the future labs and shows where the biggest amount of growth for the 
effectiveness of the laboratory lies: in motivating students to effectively communicate their information synthesis between the theory and practice found in the laboratory. Students may have experienced a lack of motivation to produce quality writing, due to the courses grading being only participation based. The best quality reports were submitted from the same groups of students consistently and vice versa. The weakest written reports were generally from the same groups of students. Professional report writing is an integral part of being a professional engineer and it is important that graduating students entering the workforce have this skill. 


\section{Conclusion}

As with all engineering problems, this issue is wrapped inside of a social issue; in this case, the need to produce innovated, independent learners capable of building successful professional careers. The uniqueness of the problem specific to this research is that this social issue also has a pedagogical component wrapped around the engineering problem. Therefore the contribution of this research to the solution of the engineering problem also addressed these pedagogical and social concerns.

\subsection{Engineering Solution}

To address the matter of physically providing an appropriate educational space, specific to augmenting the lecture course material, I designed engineering laboratory equipment specific to the lecture course. This equipment was either fabricated from these custom designs, sourced by equipment donations from industry partnerships, or purchased from vendors. The modular design and application of the equipment in the laboratory space allows for future growth in curriculum and research of power systems behavior, protection, monitoring and control through the flexibility of the modular design of the equipment.

\subsection{Pedagogical Solution}

To utilize this equipment, applicable and compelling curriculum pertinent to lecture theory was created. A modern laboratory space was implemented using equipment and software standard to the local industry and three methods of assessment were used for evaluation and improvement by the adoption of ABET SLOs, Feisel outcomes, and the 
PSRC power system protection laboratory goals for necessary curriculum topics to be covered in an introductory power systems protection course. From examination of the results of grading rubrics and surveys, I assessed that the prescribed SLOs adopted for this laboratory were met. Ultimately, the execution of the laboratory research found that the addition of the laboratory to the course contributed to the knowledge base of program graduates in the specialized topic of power system protection.

\subsection{Social Solution}

Directly addressing the need for independent learners and professionals capable of building successful careers, this laboratory contributed to the knowledge base of program graduates for the specialized topic of power system protection. Many of the current student population are actively employed in the Portland Power Pool or conducting research for PPP industry members, such as Bonneville Power Administration, PGE, Pacificorp, and POWER Engineers. The skills applied in the laboratory to successfully use industry standard equipment, design, and troubleshoot issues within a specified timeframe add to the basic abilities necessary for innovative engineering professions capable of independent lifelong learning in the power engineering sector. 


\section{References}

[1] G. Zorpette and K. Pyko, "Can power engineering education be reenergized?: Enrollments are down, leading some educators to cry 'crisis' while the industry itself skims the cream of a smaller crop," Spectrum, vol. 23, no. 12, pp. 26-31, Dec. 1986.

[2] A. Girgis, E. Makram, M. J. Cline and H. J. Fortson, "Power engineering industry interaction at Clemson University," IEEE Transactions on Power Systems, vol. 7, no. 4, pp. 1584-1590, 1992.

[3] J. Gover and P. Huray, "Educating 21st Century Engineers," in Meeting the Growing Demand for Engineers and Their Educators 2010-2020 International Summit, 2007.

[4] P. Sen, "Electric power and energy engineering education in USA: A status report, issues and challenges," in Rural Electric Power Conference (REPC), 2011 IEEE, 2011.

[5] J. Grice, M. Peer and G. T. Morris, "Today's aging workforce - Who will fill their shoes?," in 64th Annual Conference for Protective Relay Engineers, 2011.

[6] V. Heydt and G. Vittal, "Feeding Our Profession," Power and Energy Magazine, IEEE, pp. 38-45, Jan/Feb 2003.

[7] Center for Energy Workforce Development, "Gaps in the Energy Workforce Pipeline: 2011 CEWD Survey Results," 2011.

[8] Oregon and SW Washington Energy Consortium, "Top Jobs in Energy Career Guide," 2011.

[9] T. Bell, "Proven skills: the new yardstick for schools," Spectrum, vol. 37, no. 9, pp. 63-67, Sept. 2000.

[10] D. Woolston, "Outcomes-based assessment in engineering education: A critique of its foundations and practice," in Frontiers in Education Conference, 2008. FIE 2008. 38th Annual , 2008.

[11] L. Feisel, "Learning objectives for engineering education laboratories," in Frontiers in Education, 2002. FIE 2002. 32nd Annual, 2002.

[12] L. Feisel and A. J. Rosa, "The Role of the Laboratory in Undergraduate Engineering Education," Journal of Engineering Education, pp. 121-130, Jan. 2005.

[13] J. Ferris and R. Bass, "A Power Systems Protection Teaching Laboratory for Undergraduate and," in 2013 ASEE Annual Conference, Atlanta, 2013. 
[14] P. Mendoza-Araya, J. Castro, J. Nolasco and R. Palma-Behnke, "Lab-Scale TCR-Based SVC System for Educational and DG Applications," IEEE Transactions on Power Systems, vol. 26, no. 1, pp. 3-11, Feb. 2011.

[15] A. H. Montoya, "The future of the electric utility industry: Opportunities for power engineering education," in Power and Energy Society General Meeting - Conversion and Delivery of Electrical Energy in the 21st Century, 2008 IEEE.

[16] N. Hosseinzadeh and S. Senini, "An Innovative Linkage of Curricula Design, Power Engineering Industries and Universities in Queensland Australia to Promote Engineering Education," in Meeting the Growing Demand for Engineers and Their Educators 20102020 International Summit, 2007 IEEE, 2007.

[17] B. Wollenberg and N. Mohan, "The Importance of Modern Teaching Labs," Power and Energy Magazine, IEEE, vol. 8, no. 4, pp. 44,52, July-Aug. 2010.

[18] S. Mohagheghi, R. H. Alaileh, G. J. Cokkinides and A. S. Meliopoulos, "A Laboratory Setup for a Substation Scaled Model," in Power Tech Conference, Lausanne, 2007.

[19] R. Felder, "Engineering Education: A Tale of Two Paradigms." in B. McCabe, M. Pantazidou, and D. Phillips, eds., Shaking the Foundations of Geo-Engineering Education," in Shaking the Foundations of Geo-Engineering Education, CRC Press, 2012, pp. 9-14.

[20] K. A. Smith, S. D. Sheppard, D. W. Johnson and R. Johnson, "Pedagogies of Engagement: Classroom Based Practices," Journal of Engineering Education, vol. 94, no. 1, pp. 87-101, 2005 .

[21] J. Gleason and K. BoyKin , "Integrated engineering math-based summer bridge program for student retention," Advances in Engineering Education, vol. 2, no. 2, pp. 1-17, 2010.

[22] A. Johri, "Work in progress - reorganizing engineering pedagogy: Preventing student disengagement by increasing dialogic learning," in Frontiers in Education Conference, 2009. FIE '09. 39th IEEE, 2009.

[23] E. A. Eschenbach, E. M. Cashman, A. A. Waller and S. M. Lord, "Incorporating Feminist Pedagogy into the Engineering Learning Experience," in Frontiers in Education, 2005. FIE '05. Proceedings 35th Annual Conference., Indianapolis, 2005.

[24] S. Brahma, J. De La Ree, J. Gers, A. A. Girgis, S. Horowitz, R. Hunt, M. Kezunovic, V. Madani, P. McLaren, A. G. Phadke, M. S. Sachdev, T. S. Sidhu, J. S. Thorp, S. S. Venkata and T. Wiedman, "The Education and Training of Future Protection Engineers," IEEE Transactions on Power Delivery, vol. 24, no. 2, pp. 538 - 544, 2009.

[25] E. Ernst, "A New Role for the Undergraduate Engineering Laboratory," IEEE Transactions on Education, vol. 26, no. 2, pp. 49-51, May 1983. 
[26] S. Lukasik, "Industry as a partner in shaping engineering education," in Frontiers in Education Conference, 1996. FIE '96. Proceedings 26th Annual Conference, 1996.

[27] N. Mohan, "New initiatives in power engineering education at the University of Minnesota," in Power and Energy Society General Meeting, 2011.

[28] N. Mohan, "Teaching utility applications of power electronics in the first course on power systems," in Power Engineering Society General Meeting, 2003.

[29] J. L. Blackburn and T. J. Domin, Protective Relaying: Principles and Applications, 3rd ed., Boca Raton: CRC Press, 2007.

[30] S. Genheimer and R. Shehab, "The effective industry advisory board in engineering education - a model and case study," in Frontiers In Education Conference - Global Engineering: Knowledge Without Borders, Opportunities Without Passports, 2007FIE '07. 37th Annual, 2007.

[31] A. Kornecki, I. Hirmanpour, M. Towhidnajad, R. Boyd, T. Ghiorzi and L. Margolis, "Strengthening software engineering education through academic industry collaboration," in Software Engineering Education \& Training. Tenth Conference on , 1997.

[32] S. Starrett and M. Morcos, "Development of a power learning environment," in Frontiers in Education Conference, 1999. FIE '99. 29th Annual, 1999.

[33] R. Culver, R. McGrann and G. Lehmann, "Preparing students for ABET a-k," in Frontiers in Education, 2005. FIE '05. Proceedings 35th Annual Conference, 2005.

[34] E. O'Neill-Carrillo, T. Martinez-Navedo, J. Ramos, M. Velez-Reyes and E. Marrero, "Undergraduate research and new laboratory practices in power engineering," in Frontiers in Education, 2002. FIE 2002. 32nd Annual, 2002.

[35] L. S. R. Felder, "Learning and teaching styles in engineering education," Engineering Education, vol. 78, pp. 674-681, 1988.

[36] E. O'Neill-Carrillo, A. Irizarry-Rivera and M. Velez-Reyes, "Curriculum improvements in power engineering," in Frontiers in Education Conference, 2001. 31st Annual, 001.

[37] I. Huet, J. Tavares and G. Weir, "Turning Engineers into Reflective University Teachers," in Frontiers in Education Conference, 36th Annual, 2006.

[38] J. Mountain, "Work in progress - Early improvement in report writing skills using critiques," in Frontiers in Education Conference (FIE), 2011, 2011.

[39] T. Sidhu and M. Sachdev, "A graduate program in power system protection," in Power Engineering Society Summer Meeting, 2000, 2000. 
[40] T. Sidhu and M. Sachdev, "Laboratory setup for teaching and research in computer-based power system protection," in 1995 International Conference on Energy Management and Power Delivery, 1995.

[41] A. P. S. Meliopoulos, G. Cokkinides, S. Mohagheghi, Q. Binh Dam, R. Alaileh and G. Stefopoulos, "A laboratory setup of a power system scaled model for testing and validation of EMS applications," in PowerTech, 2009 IEEE Bucharest .

[42] L. R. Lattuca, P. T. Terenzini and . J. F. Volkwein, "Engineering Change: A Study of the Impact of EC2000," ABET, Inc, Baltimore, 2006.

[43] N. Schulz and W. Reder, "The challenges and opportunities of workforce development in power engineering and how the IEEE PES is helping," in 43rd International Universities Power Engineering Conference, 2008, 2008.

[44] S. Varadan, "Today's workforce in tomorrow's smart grid: Bridging the growing gaps," in Power and Energy Society General Meeting, 2012 IEEE, July 2012.

[45] IEEE, "C37.90 - IEEE Standard for Relays and Relay Systems Associated with Electric Power Apparatus," Power System Relaying Committee of the IEEE Power Engineering Society, 2005.

[46] B. Oza and S. Brahma, "Development of power system protection laboratory through senior design projects," IEEE Transactions on Power Systems, vol. 20, no. 2, pp. 532-537, May 2005.

[47] C. R. Mason, The Art and Science of Protective Relaying, Wiley, 1956.

[48] M. Sachdev, "A laboratory for research and teaching of microprocessor-based power system protection," IEEE Transactions on Power Systems, vol. 11, no. 2, pp. 613 - 619, May 1996.

[49] M. Sachdev and T. Sidhu, "A laboratory for research and teaching of microprocessor-based power system protection," IEEE Transactions on Power Systems, vol. 11, no. 2, pp. 613619, May 1996.

[50] W. Lee, J. Gu , R. Li and P. Didsayabut, "A physical laboratory for protective relay education," IEEE Transactions on Education, vol. 45, no. 2, pp. 182-186, May 2002.

[51] R. Mehta, B. Oza, N. Nair and M. Kumar, "Simulation of numerical distance protection through senior design project," in 2012 IEEE International Conference on Power System Technology (POWERCON), 2012.

[52] X. Wang, Y. Li and Y. Yu, "Research on the relay protection system for a small laboratoryscale microgrid system," Industrial Electronics and Applications (ICIEA), 2011 6th IEEE Conference on, vol. 2712, no. 2716, June 2011. 
[53] IEEE, "C37.90 - 2008 IEEE Standard for Relays and Relay Systems Associated with Electric Power Apparatus," Power System Relaying Committee of the IEEE Power Engineering Society, 2005. 
Appendix A: Revised Laboratory Assignment Instructions 


\title{
A.1 Revised ASPEN Software Introduction Instructions
}

\author{
Portland State University \\ Electrical \& Computer Engineering \\ ECE 448/548 Power System Protection I
}

\author{
-Lab 1. ASPEN Software Introduction -
}

\section{Introduction}

This lab introduces students to the power protection equipment and simulation software that will be used in subsequent laboratory assignments. The laboratory students build a single-source, three-phase radial power circuit model and a three-source, single load looped power circuit model using ASPEN software. The laboratory students review symmetrical components and will analyze the fault results by comparing simulated results to calculated results.

\section{Objectives}

At the end of the laboratory exercise the student will be able to do the following:

$>$ Create simple cases in ASPEN OneLiner, including positive-, negative- and zero-sequence impedances of line and generators as well as proper transformer connections.

$>$ Run ASPEN Power Flow on a power system case and perform basic analysis of the results.

$>$ Identify the results of the power flow that have an influence in the fault study

$>$ Perform a basic fault study, obtaining results for three-phase, single line-toground, line-to-line, and line-to-line-to-ground faults in the relevant parts of the system and interpret the results.

\section{PART 1}

\section{Setting up ASPEN}

ASPEN Overcurrent Relay Library Directory must be designated to a specific folder in your personal account.

STEP 1. From the start menu, open ASPEN Configuration window.

STEP 2. Choose a destination folder for the Overcurrent Relay Library Directory by creating a folder on your desktop and directing the configuration there, as shown in Figure 1. 
STEP3. Change the Key Type to Network access, as shown in Figure 1.

STEP4. Save setup.

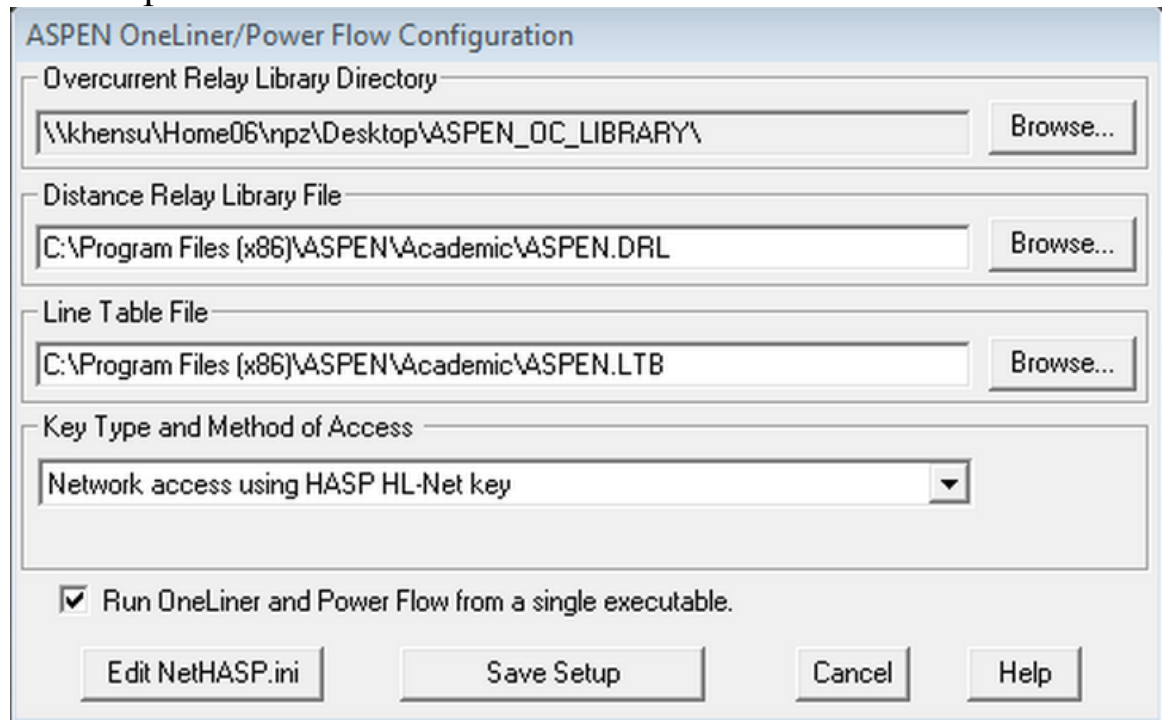

Figure 1: ASPEN Configuration window

\section{Distributed System:}

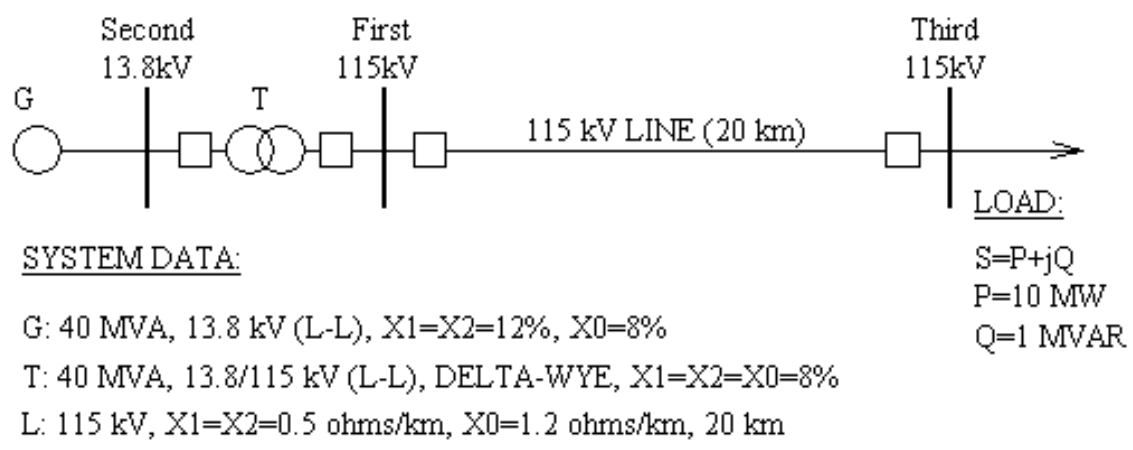

Figure 2: System diagram

STEP 1. In ASPEN OneLiner, choose an appropriate MVA base for the system, then enter the basic system data in the following order:

(Make sure the Device Palette is selected in the View tab)

1) FIRST bus $(115 \mathrm{kV})$

2) SECOND bus $(13.8 \mathrm{kV})$

3) Enter Cable line information (Neglect susceptance, B, and conductance, G.). This will automatically connect to a third bus $(115 \mathrm{kV})$. Rename this new bus as THIRD.

4) Transformer (IMPORTANT: First click the mouse on, or mark, bus FIRST and then mark bus SECOND. The first bus marked cannot contain the DELTA side of the 
transformer.) Enter the per unit values of the data listed in .

5) Add generator to SECOND bus. For transient and subtransient impedance values, use the same values as $\mathrm{X} 1$ and $\mathrm{X} 2$.

6) Add Load information (Must be entered in MW and MVAR. Do not use per unit quantities.)

7) Add the Circuit Breakers (Called "RELAY GROUPS" in OneLiner). Do not add relays to the circuit breakers.

*Note: The provided basic system data may need to be adjusted for chosen MVA ${ }_{\text {base, }}$, if MVA base differs from element MVA nominal rating.

STEP 2. Simulate a three-phase fault close to the breaker on the line side of bus FIRST. Include a snap shot of the result of the fault on the one-line diagram for phase A as well as for the sequence component currents. On the Fault Specification window, choose CLOSE-IN FAULT with NO OUTAGE and 3LG. Save the TTY results for the assignment report.

STEP 3. Simulate a single line-to-ground fault at the same point and save the same information as requested in Step 2.

STEP 4. Determine the neutral currents for both faults, at the transformer and generator neutral connections.

STEP 5. Determine accuracy of fault calculation by hand or by writing a script in Matlab using theoretical calculations.

**Reference tutorial in section 2-8 of the ASPEN OneLiner on-line help. Use the IEEE09.OLR file in the library for this tutorial if you feel you need practice before beginning the assignment.**

\section{PART 2}

\section{Looped System:}




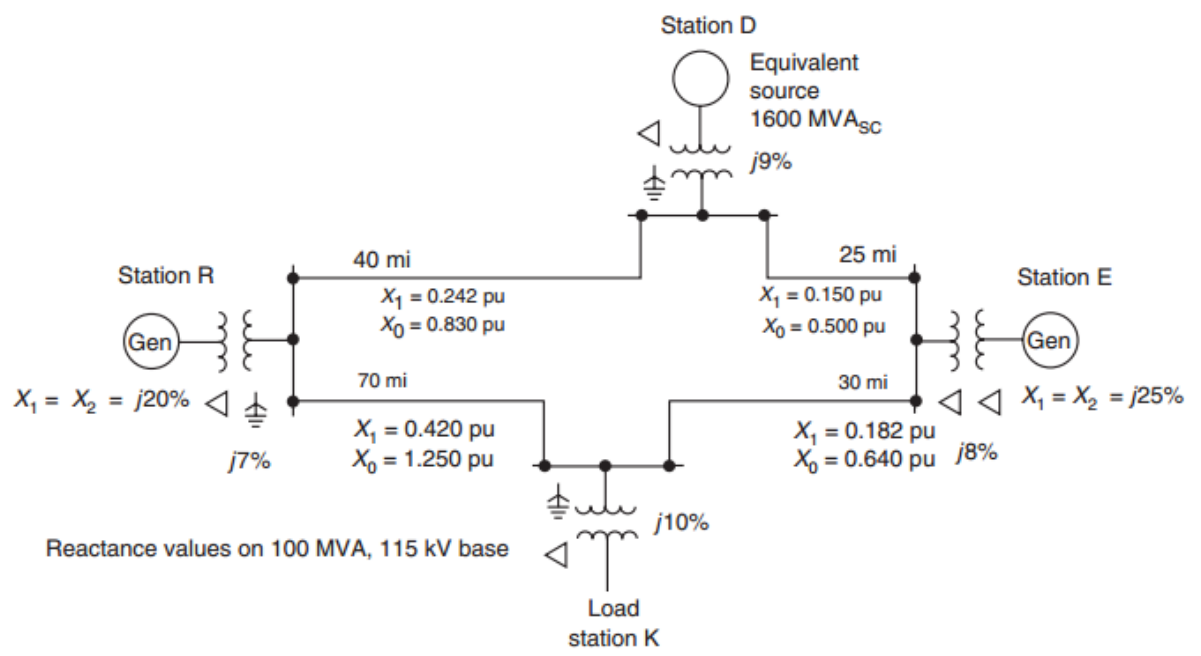

Figure 3: Power system example from Blackburn Relaying Principles (Figure 4.32)

STEP 1. Create the system from Figure 3 using in ASPEN OneLiner, adding circuit breakers to all lines and transformer ends, similar to Part 1 above. Do not click ADD button to add relays to the circuit breakers. This will be done in later labs.

STEP 2. Reference Appendix A for the complete information of system input requirements for the software.

STEP 3. Simulate three-phase and line-to-ground faults at buses in all four stations and determine the 0-1-2 sequence currents and phase a-b-c currents and voltages at each circuit breaker.

STEP 4. Add new line between stations $\mathrm{D}$ and $\mathrm{K}$ with parameters of $\mathrm{L}=100 \mathrm{mi}$, $\mathrm{X} 1=\mathrm{X} 2=0.5 \mathrm{pu}, \mathrm{X} 0=1.5 \mathrm{pu}$.

STEP 5. Repeat the simulations from STEP 3) with the additional line in the system between stations $\mathrm{D}$ and $\mathrm{K}$.

STEP 6. Simulate line-to-ground fault at the midpoint of the new line and determine the sequence 0-1-2 and phase a-b-c currents and voltages at each end of the new line. Note how the power flow results have changed with the addition of the new line.

\section{Deliverables}

Compile your results from the TTY table along with the images of the system one-lines and identify the relevant parts of the results to the fault study. Include in your report the 
information relevant to the fault analysis and write a short analysis for the fault studies regarding the results of the power flow and the effect of adding the new line between Stations D and K. Save the models for future laboratory assignments and share files with all partners. Report is due at EOB on Friday of the second week.

\section{Appendix A: ASPEN looped system component details}

\section{Station D:}

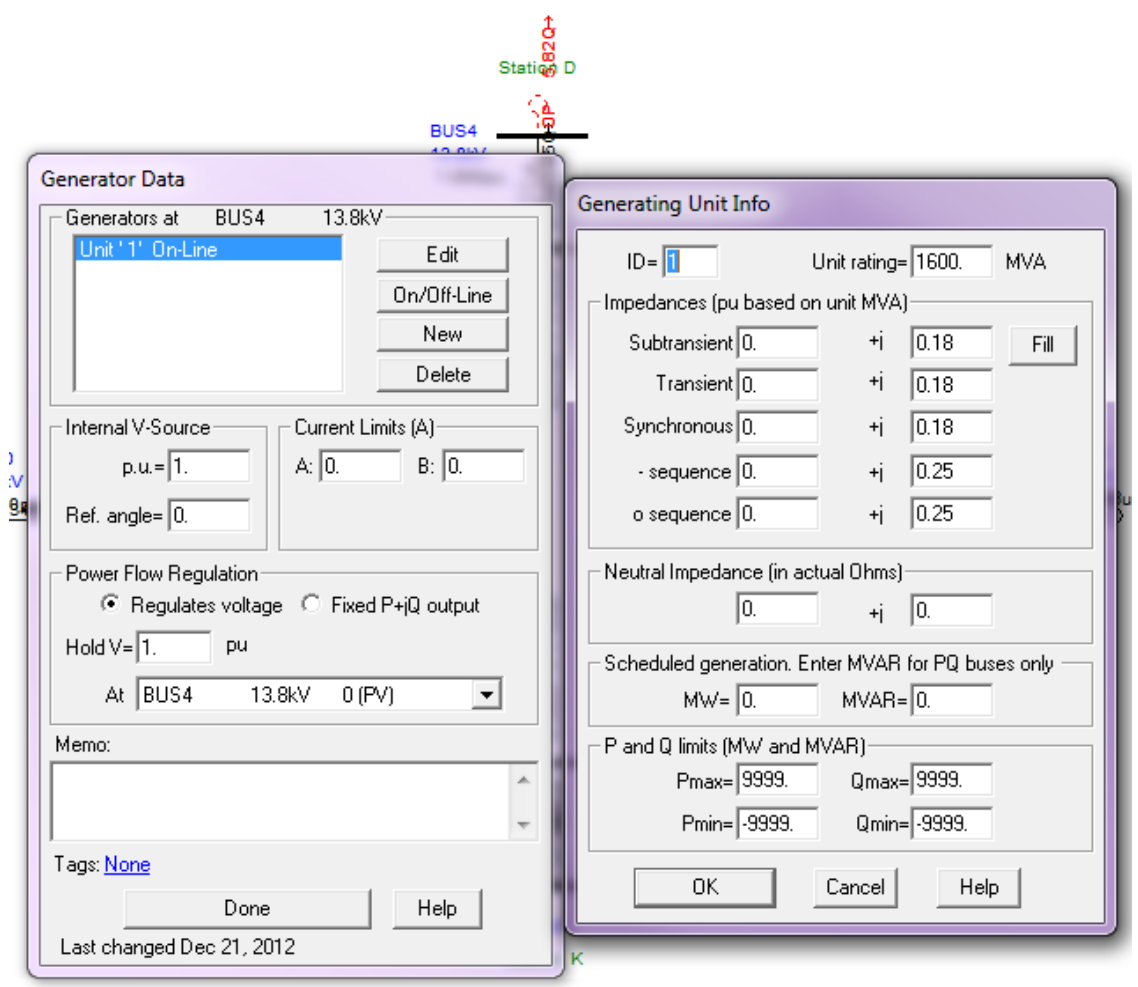




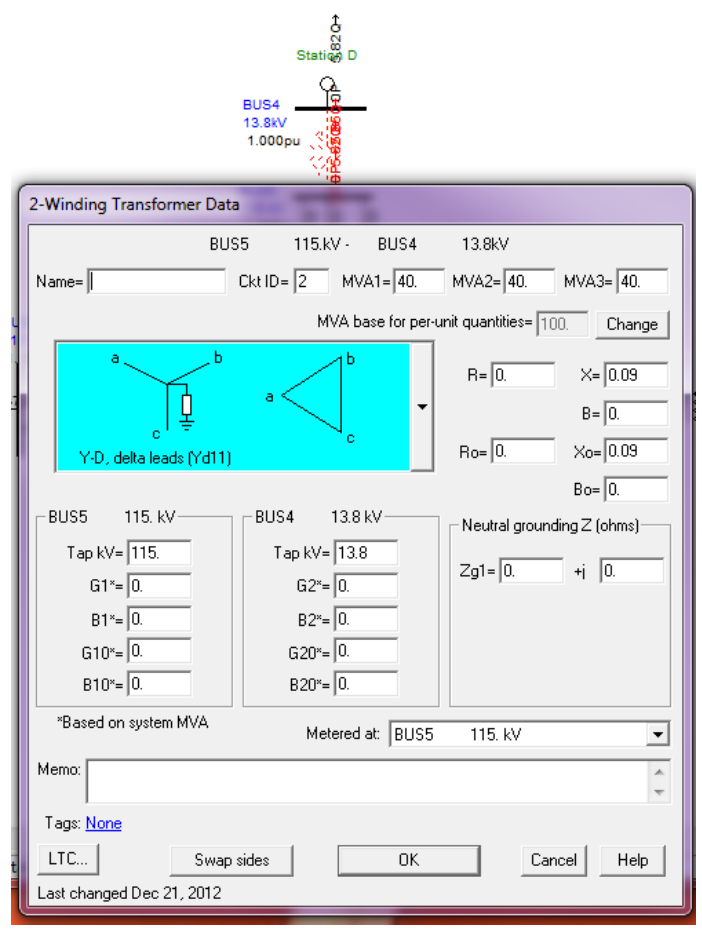

From BUS5 to BUS1:

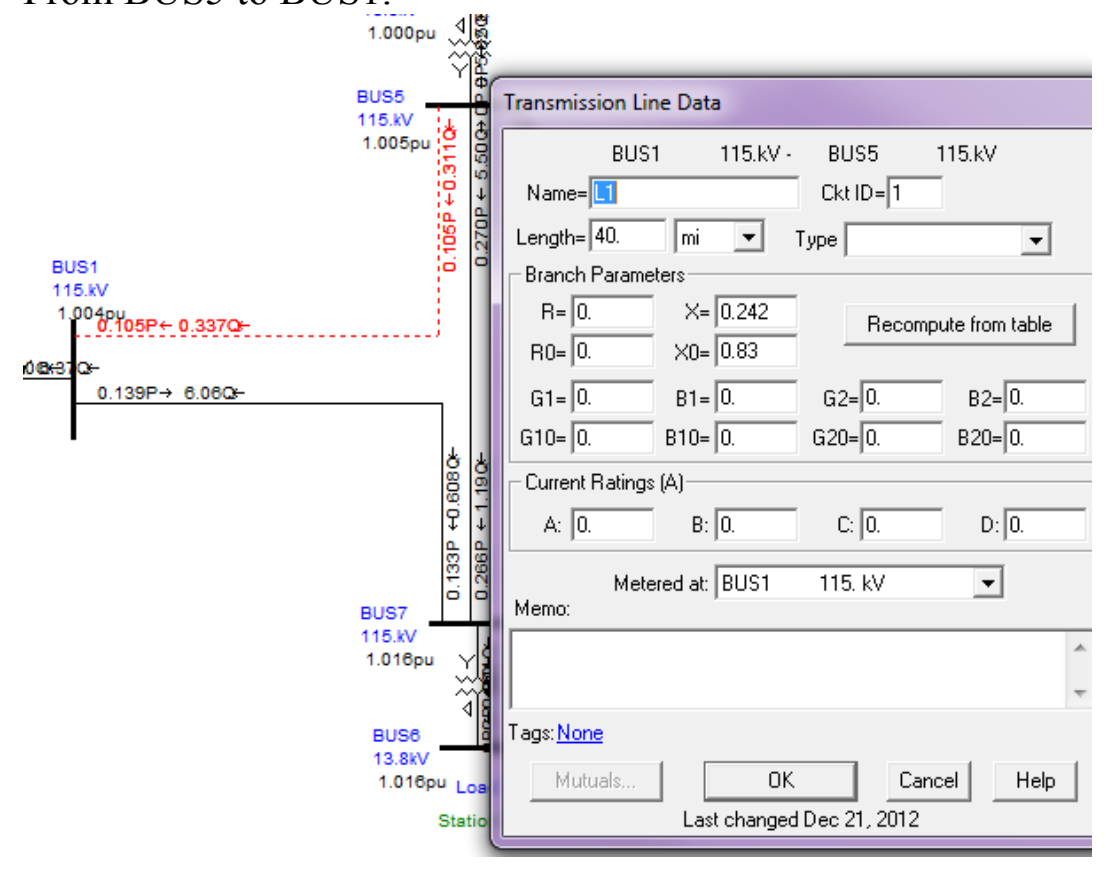




\section{Station R:}

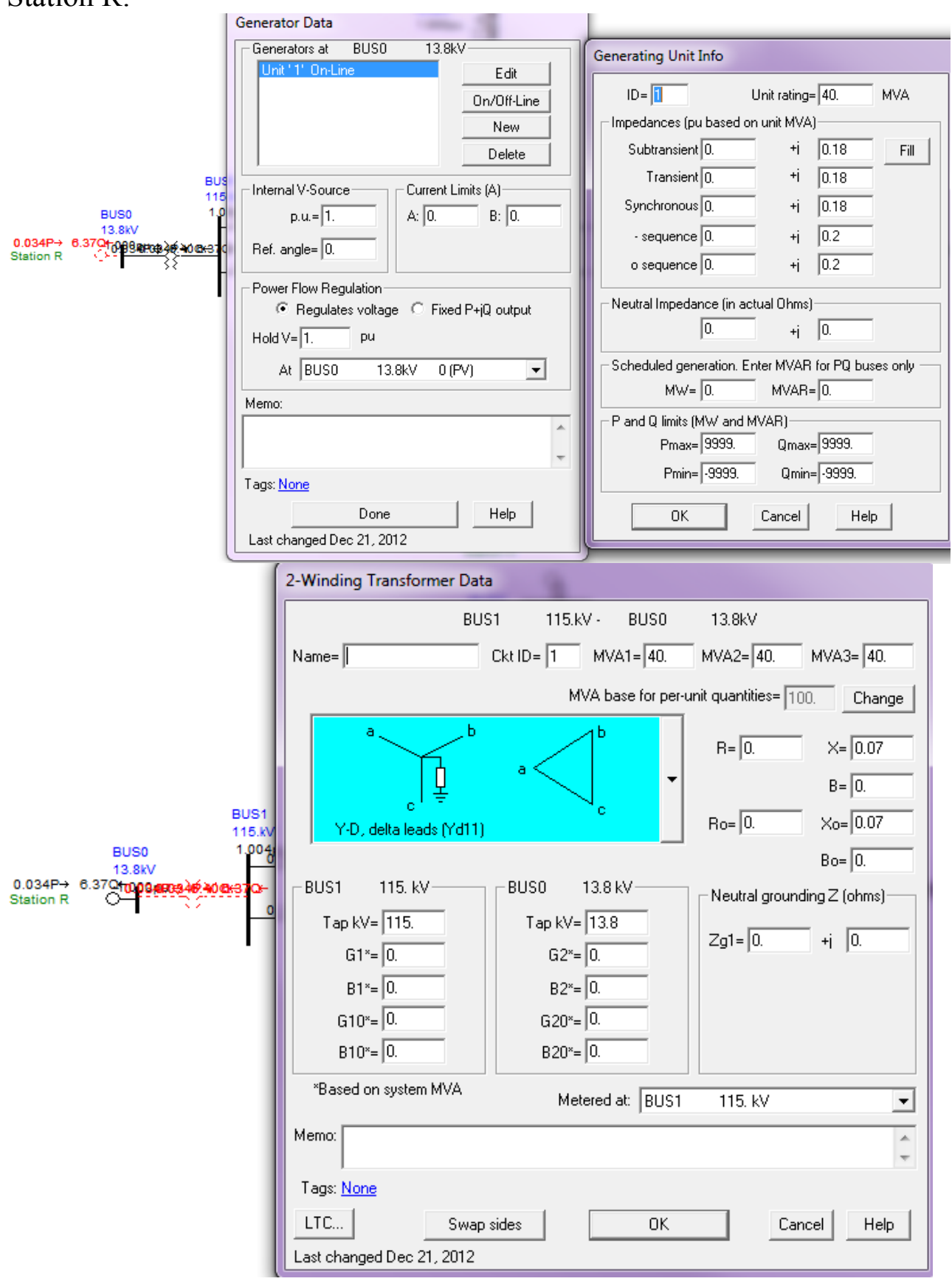


From Bus1 to Bus7:

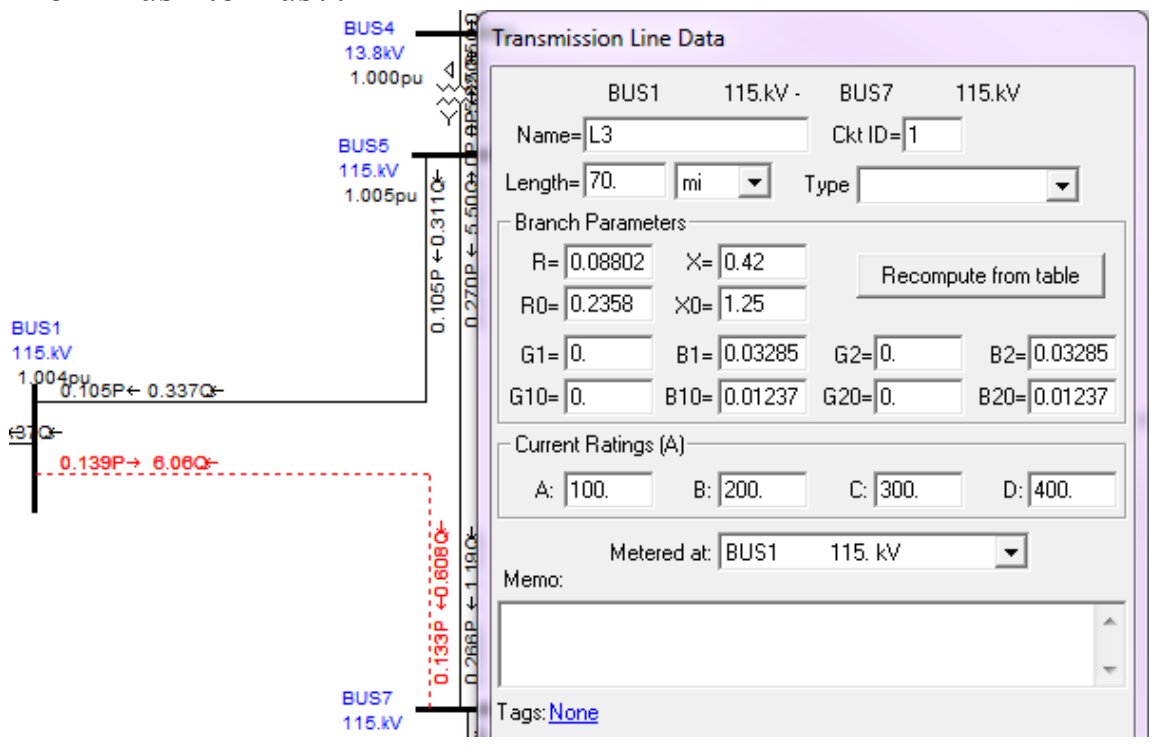

\section{Station K:}

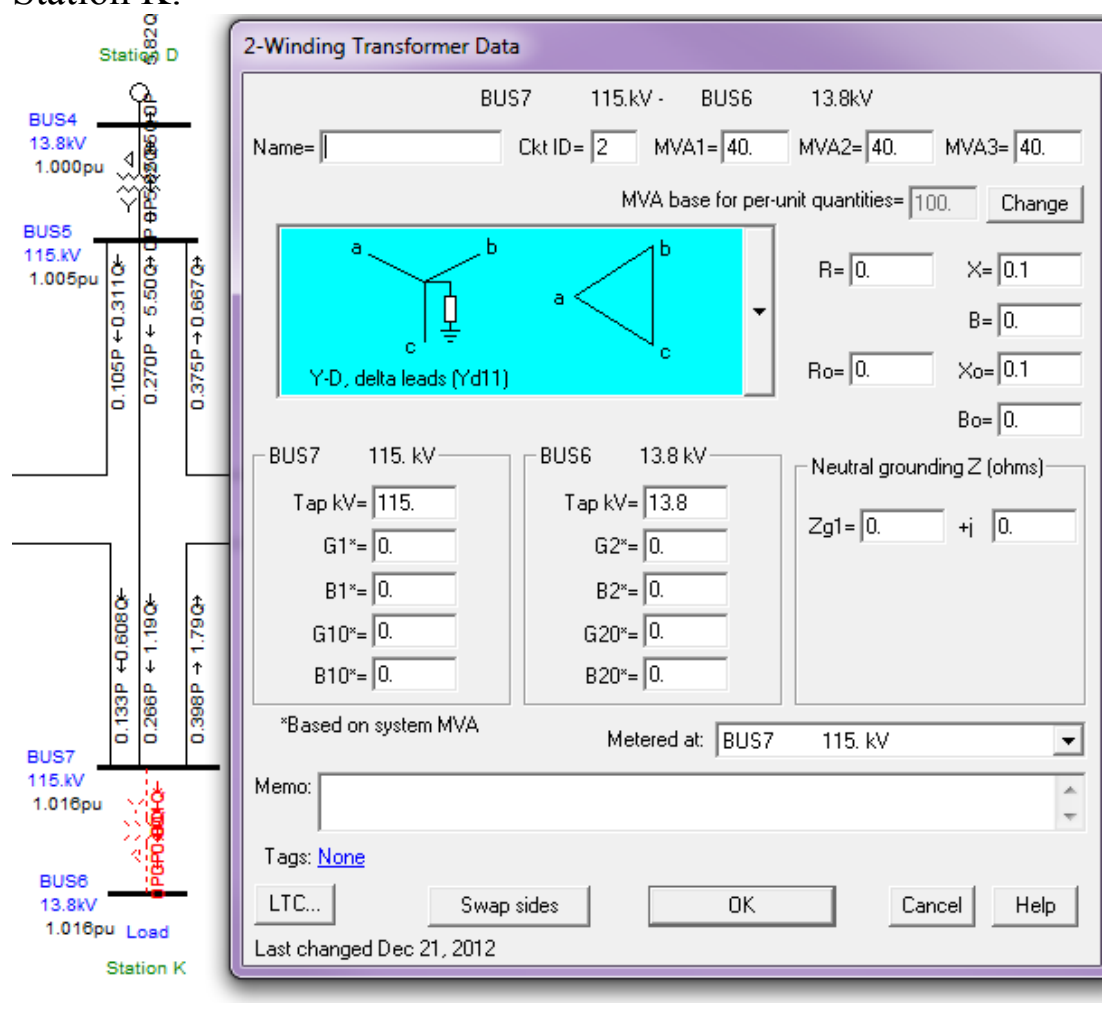


From Bus 7 to Bus3:

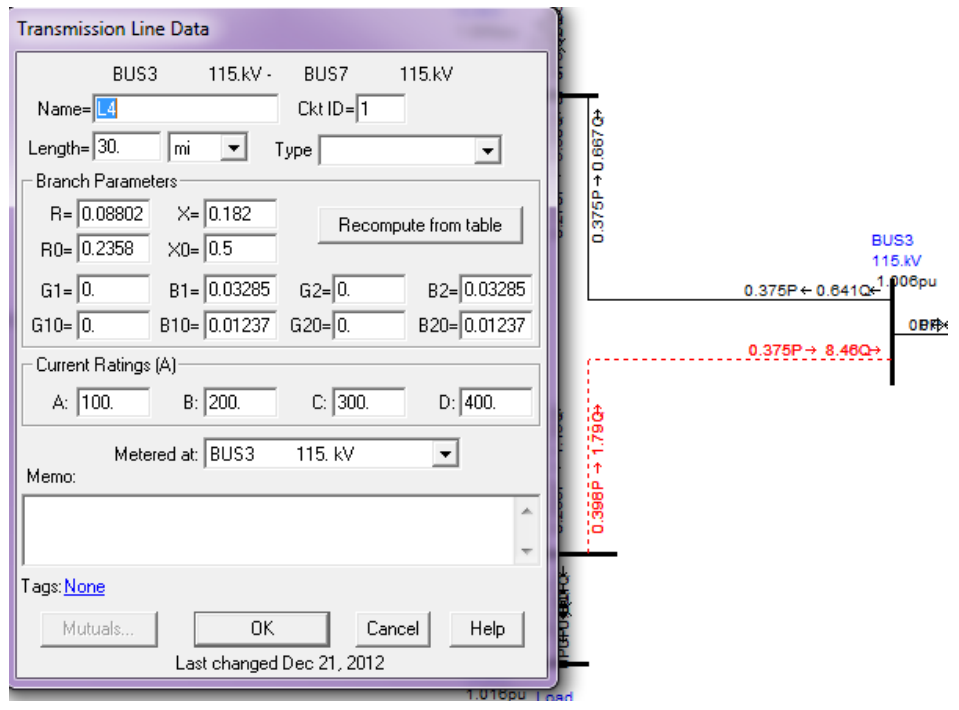

\section{Station E:}

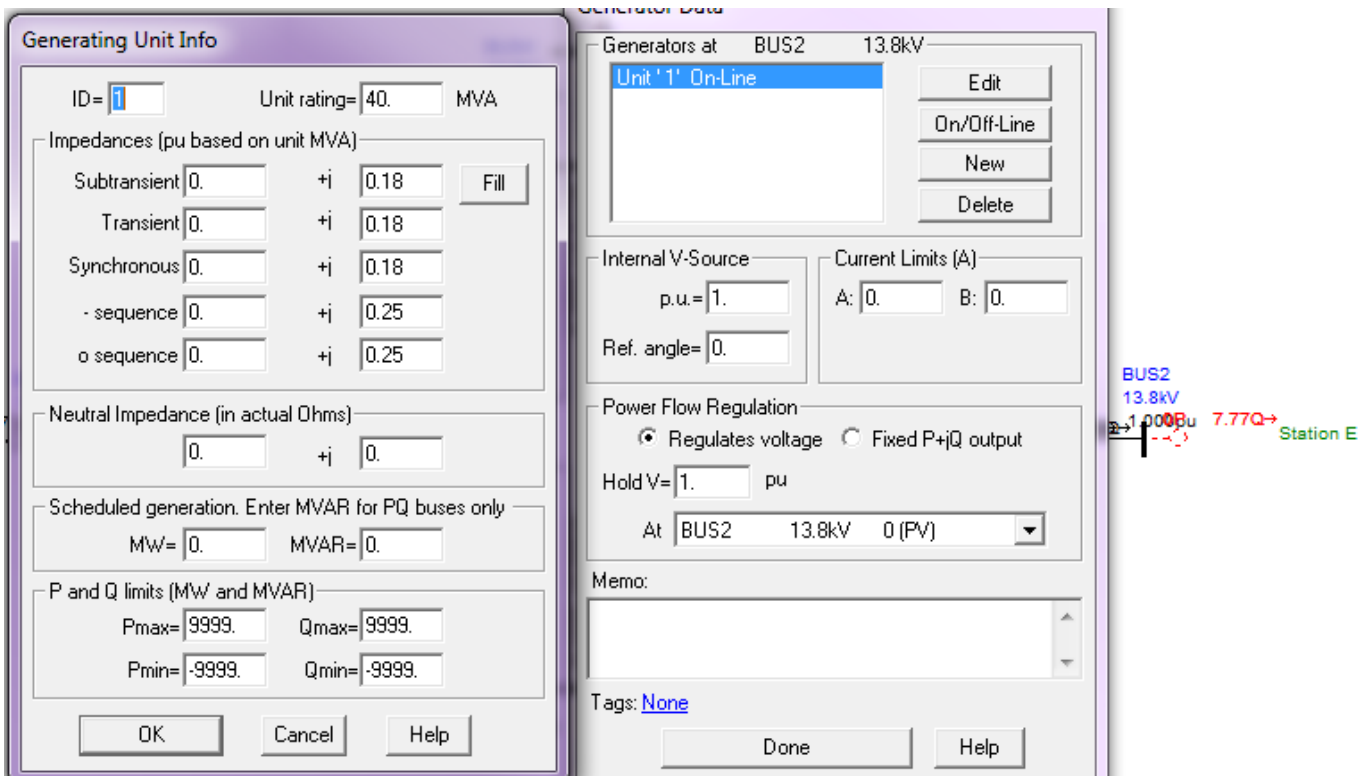




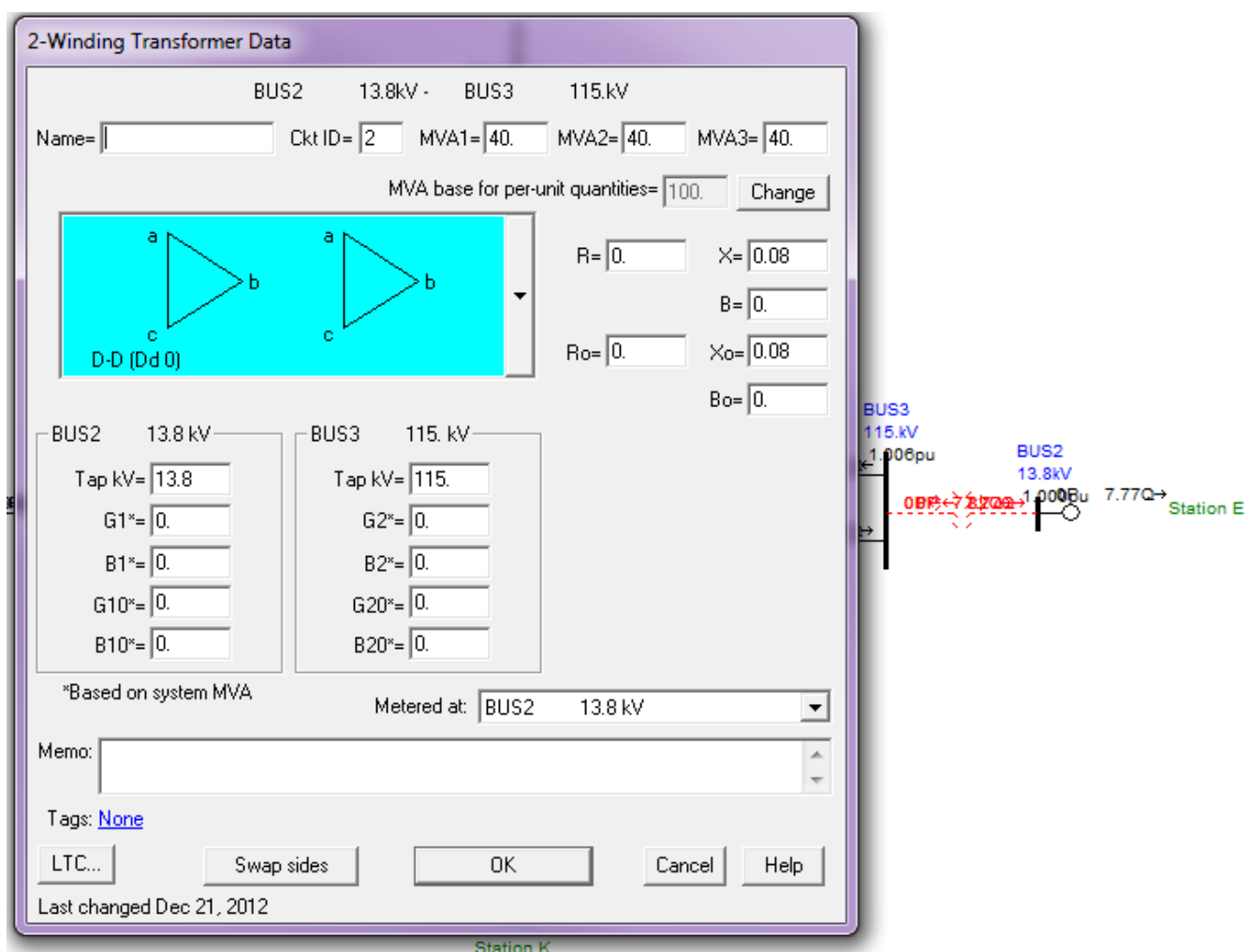




\section{From Bus3 to Bus5:}

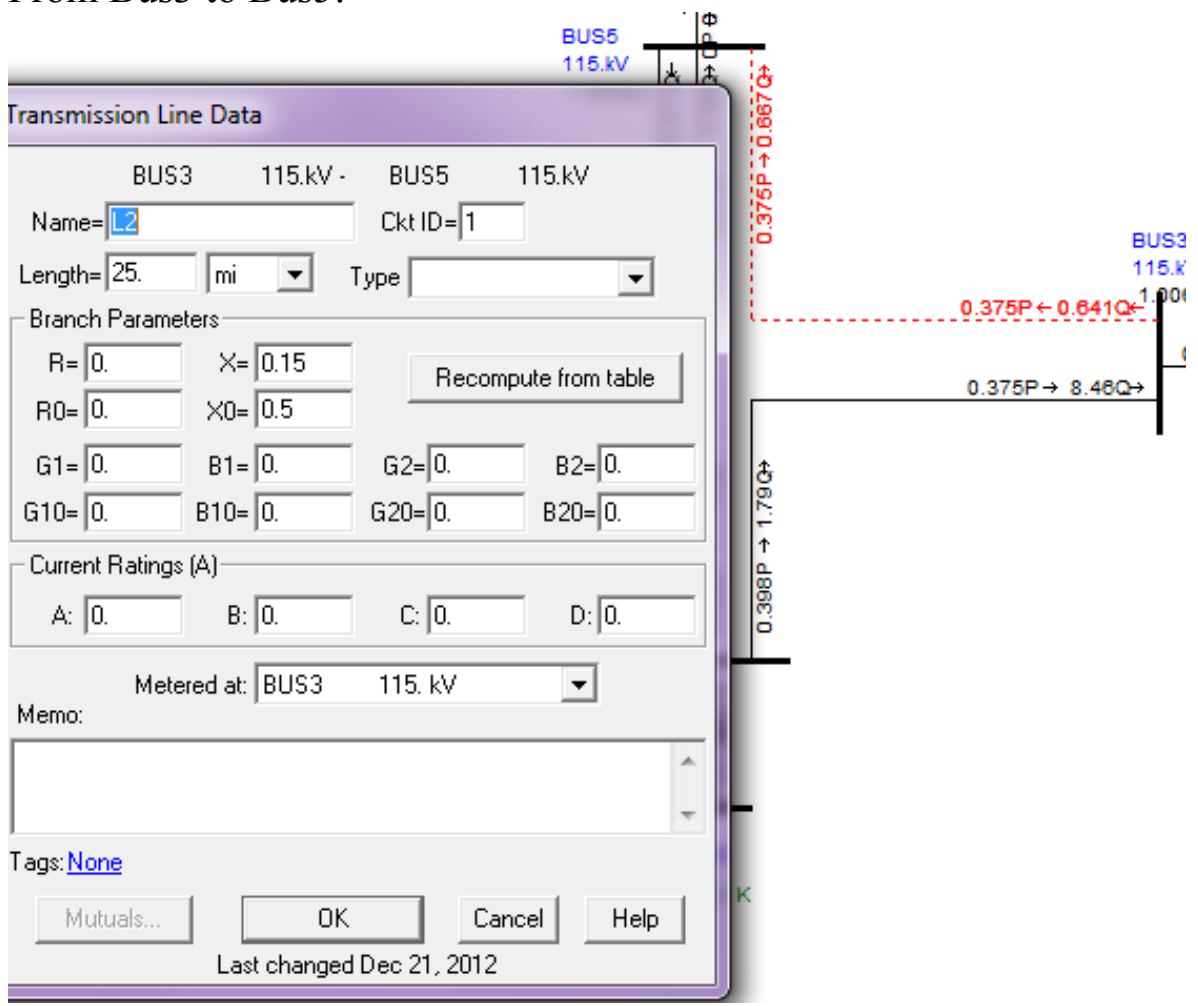




\title{
A.2 Revised Wire Heating and Fuses Instructions
}

\author{
Portland State University \\ Electrical \& Computer Engineering \\ ECE 448/548 Power System Protection I
}

\section{-Lab 2. Wire Heating and Fuses -}

\section{Introduction}

This lab introduces the student to the analysis of the dynamic heating of a wire by an electric current. The first-order differential equation will be stated and its solution (temperature vs time curve) will be compared with experimental results. In addition, the student will melt fuse wires and will construct the time-current melting curve for a fuse.

\section{Objectives}

At the end of the laboratory exercise the student will be able to do the following:

$>$ Build the temperature-vs-time heating curve of a wire in free air.

$>$ Compare the results of the experimental $\mathrm{T}(\mathrm{t})$ curve with the predicted by the single model using Matlab or other programming tool and the equation from lecture notes.

$>$ Experimentally build an approximate time-current, $\mathrm{I}(\mathrm{t})$, melting curve for the fuse.

$>$ Verify conductor material type based on thermal behavior.

D Explain the application of a time-current static curve and the dynamic temperature-time response of electrical equipment.

\section{PART 1: Determine the heating characteristics of a metallic wire in free air} Materials/Equipment

$>$ Approximately 14 inches of 8 or 10 AWG bare wire

$>$ Watch/clock with second hand

$>$ High current source: MultiAmp SR-51 or SR-76A

$>$ Appropriately rated testing leads

$>$ Multimeter with temperature probe

$>$ Oscilloscope with current probe

Heating Experiment

Determine the temperature vs. time curve for the metallic wire for two different currents 
within the ranges $20 \mathrm{~A}-30 \mathrm{~A}$ and $50 \mathrm{~A}-60 \mathrm{~A}$. Use the simple free-air model to approximate the curves obtained using Matlab.

Cut two pieces of the appropriate length of the bare 8 or 10 gauge wire provided. Attach one wire to a mounting post on fuse holder along with the appropriate testing leads. Attach the oscilloscope current probe to testing leads and prepare oscilloscope to verify measuring the testing current from the MultiAmp ammeter. BE GENTLE, DO NOT SNAP CURRENT PROBES TOGETHER. Prepare your clock by setting to zero. $* * *$ Verify set up with a TA before energizing equipment.***

$* * * \mathrm{Be}$ aware the ceramic tubes in the fuse holder stay hot for much longer than the wire. Please be careful when changing wires between tests.***

Measure the temperature of the wire through the hole in the glass of the fuse holder. Keep the temperature probe pressed on the wire in order to ensure good contact. Verify the material of the conductor using using standard metal temp vs. time curves.

Energize equipment and raise the current to a point with in the first current range simultaneously starting the clock and noting temperature at $t=0 \mathrm{sec}$. Verify the current input to the wire with the oscilloscope as well as the MultiAmp ammeter. Record the temperature every 15 seconds for the first five minutes. After the first five minutes, temperature can be recorded every 30 seconds.

Repeat this process for the second test at the higher ampere. Plot the results of the tests as well as the theoretical curves in Matlab for comparison of theoretical values to experimental values for the wire material.

\title{
PART 2: Determine the melting/clearing time curves of a fuse element
}

\author{
Materials/Equipment \\ $>$ Approximately 14 inches of 20 AWG bare wire \\ $>$ NEC Copper ampacity table \\ $>$ Watch/clock with second hand \\ $>$ Fuse holder \\ $>$ High current source: MultiAmp SR-51 or SR-76A \\ $>$ Appropriately rated testing leads \\ $>$ Oscilloscope with current probe
}

\section{Heating Experiment}

Determine the melting time vs. current curve for the fuse element (log-log) using the simple free-air model to approximate the curves obtained. Verify the material of the fuse element using the time vs. current curve and the gauge of the wire.

Verify the ampacity of the 20 AWG wire at room temperature in free air with the NEC 
copper ampacity table. Cut the four pieces at appropriate length of the bare 20 gauge wire provided. There will be four separate melting tests done to determine the $\mathrm{I}(\mathrm{t})$ curve.

Choose four levels of approximate testing current but do not exceed $60 \mathrm{~A}$, the first level of current being above the rated ampacity for $\mathrm{Cu}$ at room temperature $\left(\sim 75^{\circ} \mathrm{deg}\right)$. Attach the first wire to mounting posts on fuse holder along with the appropriate testing leads, knowing that the first test will be starting above the rated ampacity for the 20 AWG. Attach the oscilloscope current probe to testing leads and prepare oscilloscope to verify measuring the testing current from the Multi-Amp ammeter. BE GENTLE, DO NOT SNAP CURRENT PROBES TOGETHER. Prepare your clock. $* * *$ Verify set up with a TA before energizing equipment***

Starting at the lowest value of your defined range, start you clock and energize the MultiAmp testing unit, applying the first testing approximate testing current. Record this approximate value. Monitor the $\mathrm{Cu}$ wire visually, record the time when the wire turns red hot. This will be the minimum melting time. Record the time that the fuse element breaks. This will be the total melting time.

Repeat this process for the remaining three tests. Plot the results of the tests as well as the theoretical curves for comparison of theoretical values to experimental values for the wire material.

\section{Deliverables}

Produce a short report with plots of the curves and summary of results for parts 1 and 2 comparing experimental values to theoretical values. Report is due EOB on Friday, February 8th. 


\title{
A.3 Revised Auxiliary Relay Testing and CT Testing Instructions
}

\author{
Portland State University \\ Electrical \& Computer Engineering \\ ECE 448/548 Power System Protection I
}

-Lab 3. Auxiliary Relay Testing and CT Testing -

\section{Introduction}

In this laboratory exercise the students will study SC and SV type electromechanical auxiliary relays, which are currently used in the power industry and will familiarize students with the Multi-Amp relay testing equipment.

\section{Objectives}

At the end of the laboratory exercise the student will be able to do the following:

$>$ Identify the most relevant parts of the relay testing equipment

$>$ Test electromechanical auxiliary relays: pick-up and operation times

$>$ Be familiar with the features and capabilities of the electromechanical relay test equipment

\section{Auxiliary Relay Implementation}

In power protection systems, auxiliary relays are used mainly for two general functions: contact multiplication and circuit isolation. In the case of EM relaying as well as control systems there is often a need for more outputs. These outputs will be for a range of functions, including but not limited to multiple tripping, alarms, and operating other equipment, such as recording and data acquisition, and lockout. The auxiliary relays are also applied for contacts that will handle higher currents or voltages in secondary systems along with electrical and magnetic isolation of several secondary circuits. ${ }^{15}$ Due to their simplicity, testing auxiliary relays will allow students to easily become familiar with the Multi-Amp relay testing instruments and learn general concepts of relay testing and operation.

\section{Auxiliary Relay Description}

The auxiliary relays that will be tested in this exercise are SV and SC relays. These relays consist of a single instantaneous current or voltage relay and a number of contacts controlled by the operation of the instantaneous unit. Both the SV and SC use an 
electromechanical plunger relay, illustrated below in Figure 1. Each type operates in the same way, with current or voltage applied to the coil to produce flux, moving the plunger.

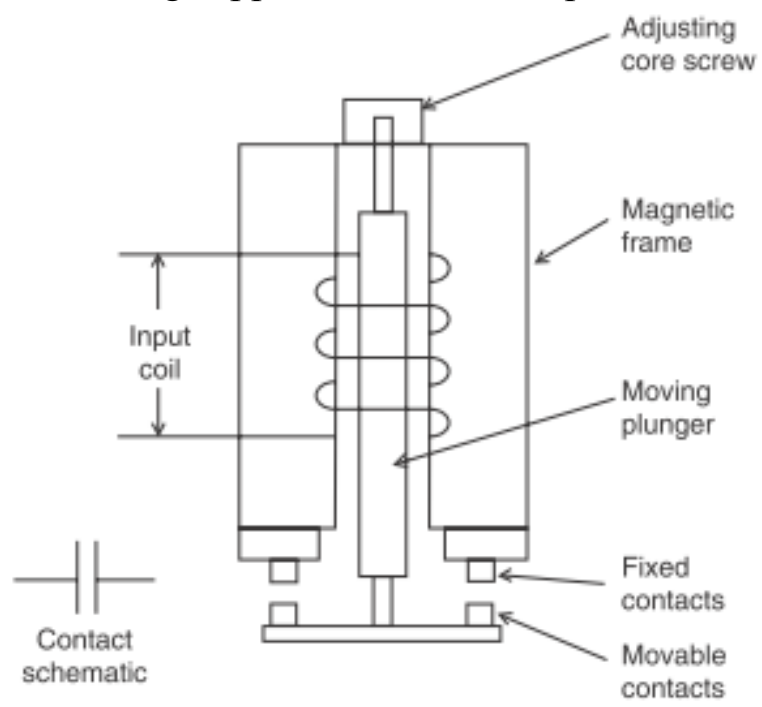

Figure 1: Typical Electromechanical Plunger Relay [Image source: Blackburn text]

\section{PART 1}

\section{Device Identification}

Remove the glass cover and physically inspect the relay, identifying the major working parts of the relays using the relay user manual. Identify the relay contact position.

\section{Testing SV and SC Relays}

Basic Connections

Figure 2 shows the internal schematic for the SC and SV auxiliary relays. The numbered terminals will be referenced below. Download the appropriate user manuals from D2L. 


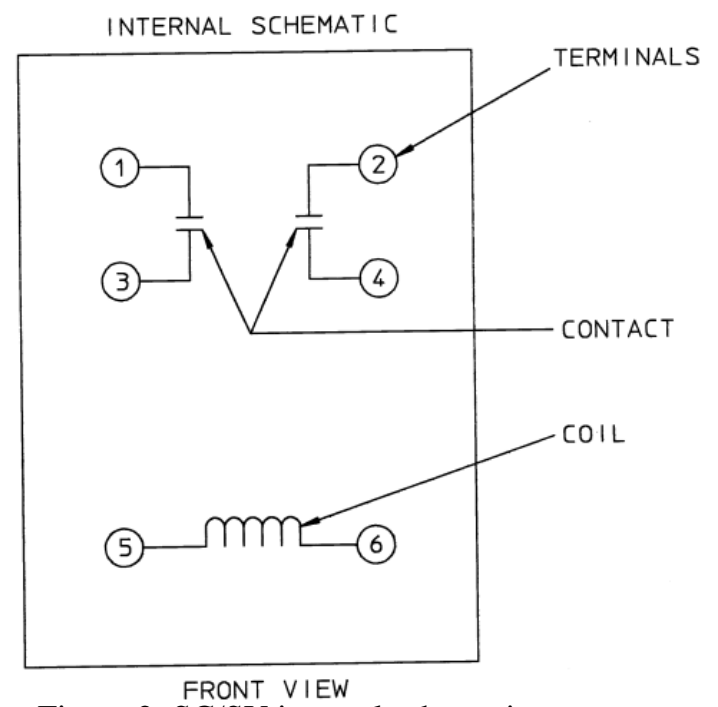

Figure 2: SC/SV internal schematic [Image source: SC/SV manual]

Note:

- Use appropriate testing leads for current being applied

- Verify type of voltage source for the SV relay

- Use an external multi-meter to verify results with analog

- Verify MultiAMP outputs are set to zero before energizing

STEP 1. Reference manuals for details regarding proper connection from the Multi AMP source output to relay operation coil terminals for the SV relays.

***for the SC relays, use the current OUTPUT listed for the IAC model relays. The manuals only list testing procedures for the SV relays, while the testing procedure is the same, the source is not***

STEP 2. Reference manuals for details regarding proper connection to relay contact terminals.

STEP 3. Consult a TA to confirm proper testing connections before continuing.

Pick Up Value Verification

This first procedure will simply verify the value that the relay operates at. Both the SC and SV relays have the same schematic and are identical in operation with the exception that the SC operates on current and the SV operates on voltage.

STEP 1. Verify pick-up value marked on core screw. Adjust core screw to different pick-up value if desired.

STEP 2. Verify that the connections are as described in the MultiAMP user manual and have been reviewed by a TA. Verify that the MAIN 
CONTROL and AUX CONTROL are dialed down to zero.

STEP 3. Follow manual instructions for testing Pick Up value verification. Verify tester settings for Pick-Up verification test with a TA before proceeding to STEP 4.

STEP 4. Energize testing unit. Push the INITIATE button, and begin to adjust the output knob slowly, watching the device meters. Once the continuity light begins to flicker, switch to adjusting the AUX CONTROL until the relay activates. Record this value and compare with value set on relay.

\section{Operation Timing}

Using the same connections and settings the relays will be tested for operation time at a number of different test instrument outputs. This procedure is not an extremely practical test, but serves to familiarize the student with the timing features of the Multi-Amp test instrument.

STEP 1. Verify that the connections and settings of the relay are identical to those used in the pick-up verification.

STEP 2. The timer controls for the relay testers are different, based on model type.

SR-51 series:

a) Make sure tester is de-energized and that relay operation coil contacts are disconnected.

b) Set TIMER OPERATION SELECTOR dial to the appropriate contact positions (NO or NC) to MOM and the toggle to CONT.

c) Based on the set pick up value of the relay, choose a target tripping value slightly higher than the pick up value.

d) Energize the unit and hold the INITIATE button as the current is ramped up to the target value. Using an external digital multi-meter may be preferred. Once the the target value is reached, release INITIATE. The value has been set.

e) Set the TIMER OPERATION SELECTOR to MAINT and the toggle to TIMER.

f) Energize tester, verify clock is set to zero and that the desired

g) units for the timer are selected. Push INITIATE button very briefly and record operation time clocked by relay. Convert time into seconds if operating in cycles.

Repeat twice. Document results.

SR-76 series:

a) Make sure tester is de-energized and that relay operation coil contacts are disconnected. 
b) Set INITIATE CONTROL dial to the appropriate contact positions (NO or NC) to NOM and the toggle to TIMER

c) Based on the set pick up value of the relay, choose a target tripping value slightly higher than the pick up value.

d) Energize the unit and hold the INITIATE button as the current is ramped up to the target value. Using an external digital multi-meter may be preferred. Once the the target value is reached, release INITIATE. The value has been set.

e) Set the INITIATE CONTROL to MAINT and the toggle to TIMER.

f) Energize tester, verify clock is set to zero and that the desired units for the timer are selected. Push INITIATE button very briefly and record operation time clocked by relay. Convert time into seconds if operating in cycles.

Repeat twice. Document results.

\section{PART 2: Current Transformer Excitation Test}

\section{Materials/Equipment}

> Veris CT data sheet for CT models AL500 and AL101

$>$ Veris CT models AL500 and AL101

$>$ Multi-Amp test unit manual

$>$ MultiAmp test unit

$>$ Oscilloscope

$>$ Current probe

$>$ Voltage Probe

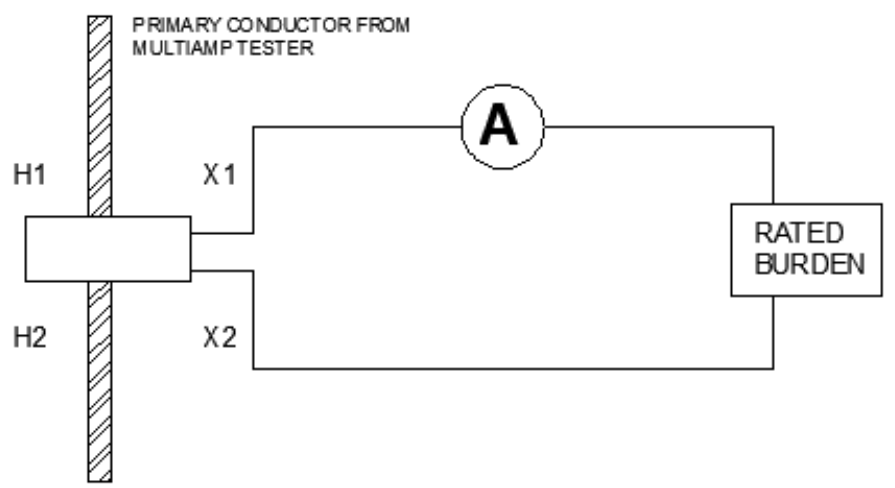

Figure 1: $\mathrm{CT}$ excitation test connection diagram 


\section{Excitation Test}

For the 50:5 CT model, run the following test: ${ }^{16}$

1) Refer to CT data sheets and course resources to determine CT accuracy class and rated burden (covert to Ohms).

2) Set oscilloscope to read voltage and current.

3) Connect the secondary CT leads to the 25 A output with the oscilloscope voltage probe and current probe attached, as shown in Figure 1.

4) Record the test voltages at intervals of approximately $0.1 \mathrm{~V}$ over a range of voltages from approximately zero up to the voltage point where the secondary current is measured at approximately $250 \%$ of rated secondary current.

5) **Never decrease the voltage during this test. If a lower voltage value needs to be tested, the test must begin from zero.**

6) Slowly decrease the voltage to zero to demagnetize the CT. Improper deenergization will lead to continued saturation of the CT core.

7) ** Note: Saturation can be reversed by re-energizing the CT back to saturation and then slowly decreasing the voltage to zero**

8) Create a log-log plot of the data and compare the results of the experimental data to the results of the manufacturer's data sheets by examining the experimental curve and calculating the approximate $\mathrm{CT}$ burden to the rated burden.

\section{PART 3: Overcurrent tests}

Materials/Equipment

$>$ Veris CT data sheet for CT model AL500

$>$ Veris CT model AL500

$>$ Rheostat

$>$ Multi-Amp test unit manual

$>$ MultiAmp test unit

$>$ Oscilloscope with two current probes

Over-current testing

The following test will be performed on the AL500 model CT only.

1) Turn on oscilloscope. Set oscilloscope to read RMS current for both current probes. Verify correct range has been set for the current probe and oscilloscope.

2) Connect the secondary leads of the AL500 to the rheostat bank.

3) Connect the $3 \mathrm{AWG}$ leads to the 0 - $100 \mathrm{~A}$ output, and appropriate ground, on the MultiAmp test unit, making sure to thread the CT through these connections.

\footnotetext{
${ }^{16}$ Excitation test procedure adapted from Back to Basics - Current Transformer Testing, C.W. Valence, http://www.netaworld.org/files/neta-journals/NWfa04-WerstiukPart1.pdf
} 
These connections serve as the primary conductor on the CT.

4) From the oscilloscope, connect one current probe around the primary conductor and one current probe around the secondary lead.

5) Verify that the control knob of the MultiAm test unit is at zero position.

6) Verify connections with a TA before proceeding

7) With TA approval, energize MultiAmp and slowly increase current output, monitoring current measurements of both sides of transformer. Verify the turns ratio experimentally under no burden.

8) Record results with screen shot of waveforms.

9) Slowly decrease the voltage to zero to demagnetize the CT. Improper deenergization will lead to continued saturation of the CT core.

\section{Deliverables}

Produce a short report with summary of results of relay tests for pick-up value verification and operation times for Part 1. Include the log-log plots of experimental data for Part 2 and oscilloscope results of the $\mathrm{CT}$ overcurrent response. Briefly discuss the results of the core saturation of the $\mathrm{CT}$ and how this relates to the rated burden. Report is due by the beginning of lab the following week. Electronic submissions are welcome. 


\title{
A.4 Revised EM Relay Radial Over-current Protection (50/51) Instructions
}

\author{
Portland State University \\ Electrical \& Computer Engineering \\ ECE 448/548 Power System Protection I
}

-Lab 4. EM Relay Radial Over-current Protection (50/51)-

\section{Introduction}

In this laboratory exercise the students will be introduced to common electromechanical over-current relays currently used by the power industry.

\section{Objectives}

At the end of the laboratory exercise the student will be able to do the following:

$>$ Identify the most important part of an electromechanical over-current relay

$>$ Explain the application of each part of the relay

$>$ Determine the pick-up current of the inverse-time relay element using a relay testing equipment

$>$ Construct the time-current curve of the EM relay

$>$ Determine the instantaneous current of the inverse-time relay element using a relay testing equipment

$>$ Compare the results with the characteristics given by the manufacture in the relay's manual

\section{Materials/Equipment}

$>$ MultiAmp relay tester

$>$ Test leads

$>$ Oscilloscope

$>$ Current probe

$>$ IAC 53B

$>$ IAC 77B

$>$ Relay curves for both relay types 

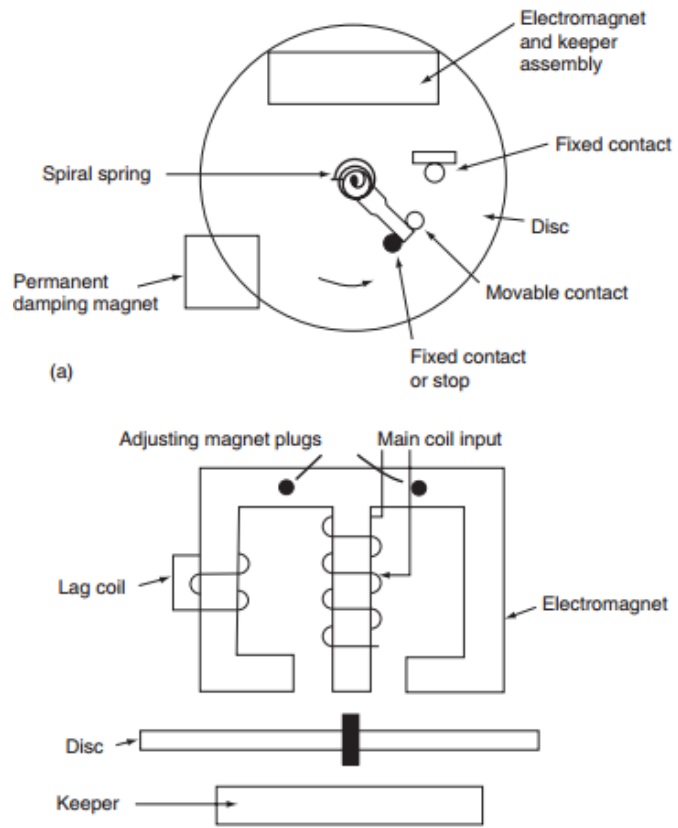

Figure 1: Induction disk construction schematic [Image source: Blackburn text]

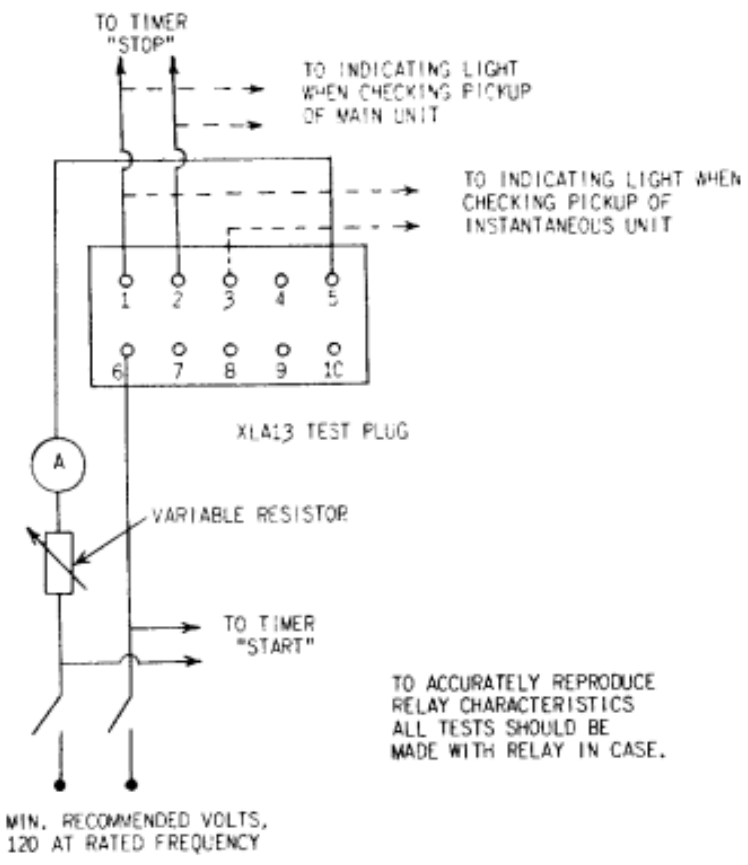

Figure 2: IAC relay test connections for pick-up and time curve settings [Image source: GE IAC relay manual] 


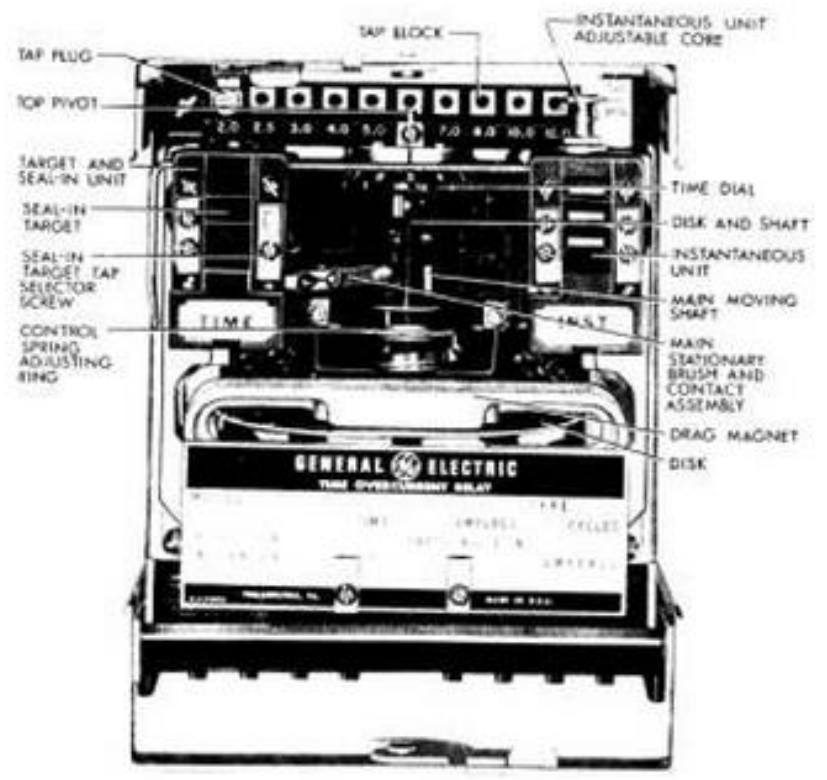

Figure 3: IAC relay elevation description [Image source: GE IAC relay manual]

\section{Basic Connections}

Figure 2 shows the internal schematic of the IAC relays. The numbered terminals in the schematic will be referenced below:

1) Connect a current source from the MultiAmp unit to relay terminals 5 and 6 .

2) Connect the Relay Contact posts to relay terminals 1 and 2 for time delay.

Connect relay terminals 1 and 3 for the instantaneous element.

\section{PART 1: Testing IAC 53B relay elements (Very Inverse)}

STEP 1: Download IAC53B manual and identify most relevant parts of the relay STEP 2: Download MultiAmp manual.

STEP 2: Reference MultiAmp manual Table of Contents for relay type. Find relay type. Go to section and read instructions for basic relay testing procedure outlined there.

STEP 3: Connect oscilloscope to relay testing circuit.

STEP 3: Perform Pick-up Test on the relay. Record results and compare to settings.

Note the difference between the MultiAmp ammeter and the oscilloscope readings. 
STEP 4: Perform Time Current Characteristic test. Record results for time and current. Note the difference between the MultiAmp ammeter and the oscilloscope readings. Compare to calculated operating time values using equations from lecture material. Plot both results on provided relay curve from relay manual.

STEP 5: Perform Instantaneous Operation test. Record result and compare to physical settings.

\section{PART 2: Testing IAC 77B relay elements (Extremely Inverse)}

STEP 1: Download IAC77B manual and identify most relevant parts of the relay STEP 2: Download MultiAmp manual.

STEP 2: Reference MultiAmp manual Table of Contents for relay type. Find relay type. Go to section and read instructions for basic relay testing procedure outlined there.

STEP 3: Connect oscilloscope to relay testing circuit.

STEP 3: Perform Pick-up Test on the relay. Record results and compare to settings.

Note the difference between the MultiAmp ammeter and the oscilloscope readings.

STEP 4: Perform Time Current Characteristic test. Record results for time and current. Note the difference between the MultiAmp ammeter and the oscilloscope readings. Compare to calculated operating time values using equations from lecture material. Plot both results on provided relay curve from relay manual.

\section{Deliverables}

Produce a short report with summary of results, including operation time calculations and the hand plotted results on the Time-Current curves, for parts 1 and 2 comparing experimental values to theoretical values. Report is due EOB on Friday, February 22nd. 


\title{
A.5 Revised Coordination of OC Relays in Radial Systems Instructions
}

\author{
Portland State University \\ Electrical \& Computer Engineering \\ ECE 448/548 Power System Protection I
}

-Lab 5. Coordination of OC Relays in Radial Systems-

\section{Introduction}

In this laboratory exercise the students will be introduced to the coordination of overcurrent relays in a radial power system using an industry software program (ASPEN OneLiner).

\section{Objectives}

At the end of the laboratory exercise the student will be able to do the following:

$>$ Create a radial case in ASPEN OneLiner containing the required information to do fault studies and overcurrent relay coordination

$>$ Introduce data of phase and ground overcurrent relay elements into the model

$>$ Simulate faults and determine the behavior (time) of overcurrent relay elements

$>$ Perform a coordination study to determine the relay settings

\section{Distributed System:}

Using the distributed system model from Lab 1, re-save model under new name and make the following alterations:

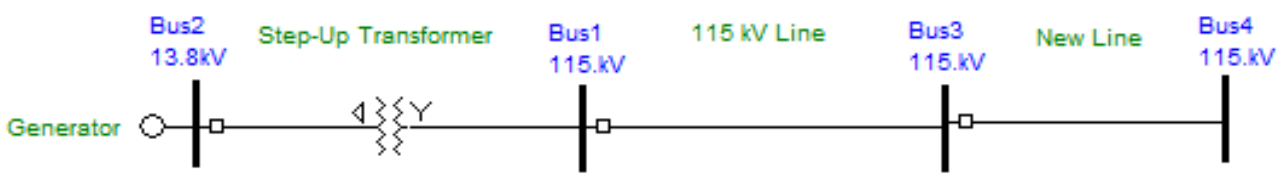

System Data:

G: 40 MVA, $13.8 \mathrm{kV}$ (L-L), X1=X2=12\%, X0 $=8 \%$

$\mathrm{T}$ : 40 MVA, $13.8 / 115 \mathrm{kV}$ (L-L, Dy11), X1 $=\mathrm{X} 2=\mathrm{X} 0=0.2$ (for 100 MVA system base)

$\mathrm{L}: 115 \mathrm{kV}, \mathrm{X} 1=\mathrm{X} 2=0.5 \mathrm{ohms} / \mathrm{km}, \mathrm{X} 0=1.2 \mathrm{ohms} / \mathrm{km}$ (adjust values to $Z_{\mathrm{pu}}$ )

Figure 1: ASPEN radial system model 
STEP 1. Simulate a 3LG fault at the same point as done in Lab 1, near Bus1 on the 20 $\mathrm{km}$ line side, to affirm the results found in lab 1 are still the same. Record fault current values of simulation with a figure of the model. Verify these results with TAs to make sure your system model is correct.

STEP 2. Delete the load on the Bus 3 and add new $10 \mathrm{~km}, 115 \mathrm{kV}$ line between Bus3 (program will automatically create the new Bus 4) with the following impedances:

$$
\begin{aligned}
& >\mathrm{Z}_{\mathrm{L} 1}=\mathrm{Z}_{\mathrm{L} 2}=\mathrm{j} 0.1 \text { p.u. } \\
& >\mathrm{Z}_{\mathrm{L} 0}=\mathrm{j} 0.3 \text { p.u. }
\end{aligned}
$$

STEP 3. Insert a circuit breaker located at Bus3 on the $10 \mathrm{~km}$ line side, as shown in Figure 1, using the Relay $\rightarrow$ New Relay Group.

STEP 4. Insert a group of phase relays in the circuit breaker located at Bus3 on the $10 \mathrm{~km}$ line side by selecting the circuit breaker and choosing the OC Phase Relay under Properties $\rightarrow$ Add (highlight $\mathrm{CB}$ and right click). Set the following characteristics for the relay:

ID:

R1

Relay Type:

CT Ratio:

Ipu:

Time Dial:

Instantaneous:
General Electric IAC53

$$
400 / 5=80
$$

\section{A mp}

3

100,000 Amps

STEP 5. Insert a group of phase relays in the circuit breaker located at Bus 1 of the 20 $\mathrm{km}$ line side:

ID:

R2

Relay Type:

General Electric IAC53

CT Ratio:

$600 / 5=120$

Ipu:

5 Amp

Time Dial:

1.5

Instantaneous:

100,000 Amps 
STEP 6. Again, using the same procedures as in Lab 1, simulate a three phase fault close to the circuit breaker located close to Bus 3 on the $10 \mathrm{~km}$ line side. Examine and record the fault currents on the one-line diagram with a figure.

STEP 7. Using the same procedure that produced the fault currents on the one-line diagram, display all relay operation times by choosing the clock icon on the program toolbar. Examine and record the relay operation times on the one-line diagram with a figure. Analyze results and determine if the relay operation sequence is correct. Compare analysis determination with relay curves.

STEP 8. Choosing Relay Curves in the Relay menu, display the relay curves at the marked relay group (circuit breaker). Use the Add command in the Relay Curves window to Add Relay Curves and add the relay from the other relay group. Under the Show menu choose Relay Operations for 1 Fault. Record the relay curves for this fault with a figure. Verify coordination with the curves, adjusting settings for proper coordination, if necessary.

STEP 9. Add another IAC53 relay on the $13.8 \mathrm{kV}$ side of the transformer near Bus2. Calculate the $\mathrm{CT}$ rating using $\mathrm{I}_{\mathrm{sc}}$ of Bus 2 provided in Table 1 above and choose appropriate rated CTR for the phase relay. Choose $\mathrm{I}_{\mathrm{pu}}=5 \mathrm{~A}$ for this new relay and calculate the time dial settings for a proper coordination with the rest of the system and put your systems in the ASPEN relay data window. Plot the relay and the transformer damage curve together with the curves of the rest of the relays. Verify coordination graphically with these curves. Write your conclusion with regard to coordination result.

\section{Deliverables}

Produce a report with an analysis of the relay coordination, including any coordination issues and solution results as well as the figures of system results and relay curves detailed in steps 1 through 9 . Report is due by email at the EOB on Friday, March $1^{\text {st }}$. 


\title{
A.6 Revised Digital Relay Radial Over-current Protection (50/51) Instructions
} Portland State University

Electrical \& Computer Engineering

ECE 448/548 Power System Protection I

\author{
-Lab 6. Digital Relay Radial Over-current Protection (50/51)-
}

\section{Introduction}

In this laboratory exercise the student will be introduced to the SEL-551 relay. It will be done using the SEL-RTS relay testing equipment.

Objectives

At the end of the laboratory exercise the student will be able to do the following:

$>$ Implement physical set-up to test SEL-551 using the SEL-RTS (AMS)

$>$ Communicate with the relay using the SEL-AMS

$>$ Introduce settings to the relay (Phase and ground elements)

$>$ Properly set a test using the SEL-5410 software

$>$ Test the relay with the AMS and obtain operation times

$>$ Verify operation results by comparing with results found under software simulation

Materials/Equipment

$>$ SEL-AMS

$>$ SEL-5401

$>$ AcSELerator QuickStart software

$>$ SEL-551

$>$ C750A ribbon cable

$>$ C734A serial cable 


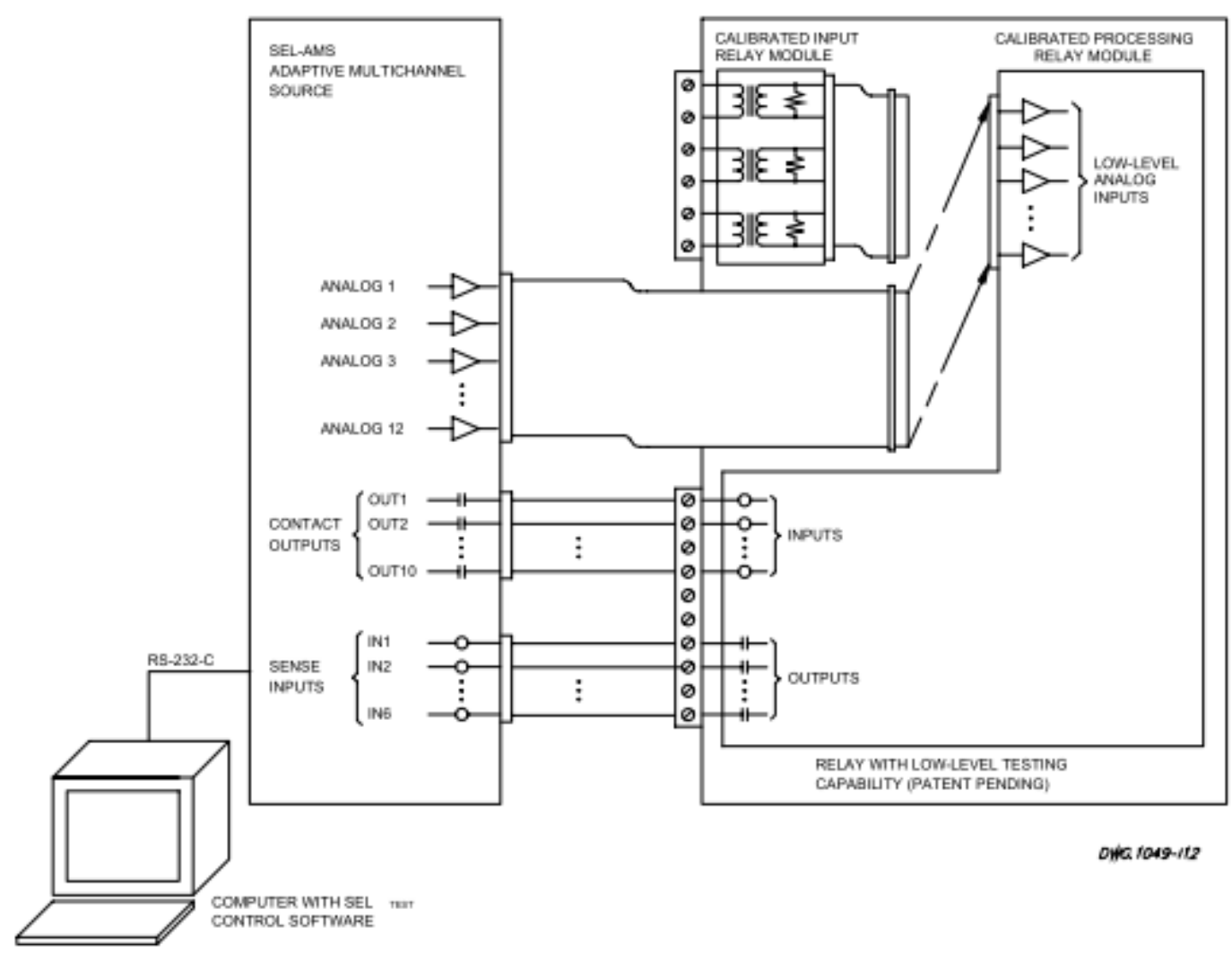

\section{Instructions}

Figure 1: Digital relay testing physical set-up with SEL-AMS

STEP 1: Use the AcSELerator software to set the SEL-551 relay. Reference Appendix A for instructions.

STEP 2: Open the Instruction Manuals for the SEL-AMS and SEL-551. Verify that the electrical connections from the SEL-AMS to the SEL-551 are correct using the wiring diagram available in the manual of the SEL-AMS.

STEP 3: To verify SEL-AMS is handshaking with PC, in the SEL-5401 software, go to File $\rightarrow$ Open $\rightarrow \mathrm{C}: / / \rightarrow$ Program files $(\mathrm{x86}) \rightarrow$ SEL $\rightarrow$ SEL-5401 $\rightarrow$ Data $\rightarrow$ SAMPLE.RTA, open this RTA file. Download and run this test. When red light on Contact Output Status 1 turns red and the SEL - 5401 window Sense Input shows a $67 \mathrm{sec}$ trip, you know you have a communication between devices.

STEP 4: File $\rightarrow$ New Relay Config window appears, go to New relay, UUT Database window appears, choose Relay Type $\rightarrow$ SEL-551. In the UUT File Selection box, choose 1 Amp relay file and Apply. Back to Relay Config window, go to $\mathbf{1 4 . 0 0}$, if so choose $\rightarrow$ Okay 
Begin setting States up in the Standard tab defining phase current values as 1004 /CTR A (the same as close-in fault on Bus1 in Lab 6). Contact Outputs for OUT1 and OUT2 set to ('c'). Set INPUTS --> IN1 to $\mathbf{C}->\mathbf{O}$. **Record Relay Config window settings as a figure for your report

STEP 5: Download and run the program on the relay. Open UUT report to view and record results for the lab report.

\section{Deliverables}

A written lab report is due complete with figures of Relay Configuration window, the UUT report results, and the printed page of relay settings from AcSELerator software for relay. A discussion of the tested results compared to the expected results characterized by the operation time of relay R2 in the ASPEN simulated model from Lab 6 is also required for this written report. The report is due EOB on Friday, March 8, 2013.

\section{Appendix A：SEL-551 Setting Instructions}

\section{Instructions}

Step 1: Go to selinc.com and register for a free account. Once you have an account, search the SEL website for the AcSELerator software download and install it on your personal computer.

Step 2: Open SEL AcSELerator Quickset software $\rightarrow$ Settings $\rightarrow$ New In the Settings Editor selection, choose SEL-551 $\rightarrow$ SEL-551 $\rightarrow \mathbf{0 0 2}$ and then Okay.

Step 3: The Device Part Number will appear. Change Communications Port to $\mathbf{2}=$ EIA-485

\section{Group 1 Settings -}

- Identifier Labels - leave as default

- CTR: 160

- Min Trip Duration Timer: 9.000 cycles (default, refers to Reclosing feature not being used in this lab.)

- Set Phase Instantaneous Overcurrent Elements 50P1-50P6 to OFF

- Set Single-phase Instantaneous Overcurrent Elements 50A, 50B, 50C to OFF

- Set Phase Time-Overcurrent Element 51P1T setting to your own Lab 6 settings designed for R2.

- Relay curve is U3.

○ Ipu = Ipu_R2_final of Lab 6 relay coordination settings

○ $\mathrm{TD}=\mathrm{TD} \_\mathrm{R} 2 \_$final of Lab 6 relay coordination settings 
○ Set 51P1RS Phase Time-Overcurrent EM Reset to N

- Set Phase Time-Overcurrent Element 51P2T to OFF, leave all other settings as default

- Set Neutral Ground Instantaneous Overcurrent Elements 50N1, 50N2 to OFF

- Set Neutral Ground Time-Overcurrent Elements 50N1T to OFF

- Set Residual Ground Instantaneous Overcurrent Elements 50G1, 50G2 to OFF

- Set Residual Ground Time-Overcurrent Elements 50G1T to OFF

- Set Negative Sequence Instantaneous Overcurrent Elements 50Q1, 50Q2 to OFF

- Set Negative Sequence Time-Overcurrent Elements 50Q1T to OFF

- Set Negative Sequence Time-Overcurrent Elements 50Q2T to OFF

- Reclosing Relay Open Interval Timer - leave as default

- Reclosing Relay Reset Timer - leave as default

- Close Failure Time - leave as default

- Demand Ammetering Settings - leave as default

- SELogic Variable Timers - leave as default

- Other System Parameters - leave as default

Print your settings by going to File $\rightarrow$ Print $\rightarrow$ Standard. In Standard Print Settings Report window, File $\rightarrow$ Print All and include in Lab 7 report.

Extra

Student may also download SEL-5401 testing software at their discretion. This is the SEL relay testing software. It is the program that will be used on the lab PCs to interface with the relay and directly test your relay settings. The student may wish to explore the SEL-5401 software before their assigned laboratory section, but this step is not required, it is left to the student to decide how much further they wish to investigate this pre-lab.

Manuals for the SEL-AMS and SEL-551 are uploaded to D2L. There is hard copies of the manual for the SEL-AMS in the lab. 


\title{
A.7 Revised Protection Coordination of Looped Systems Instructions Portland State University Electrical \& Computer Engineering ECE 448/548 Power System Protection I
}

\author{
-Lab 7. Protection Coordination of Looped Systems-
}

\section{Introduction}

In this laboratory exercise the students will be introduced to the coordination of overcurrent relays in a looped power system using a industry software program (ASPEN OneLiner).

Objectives

At the end of the laboratory exercise the student will be able to do the following:

$>$ Create a looped case in ASPEN OneLiner containing the required information to perform fault studies and overcurrent relay coordination.

$>$ Introduce data of phase and ground directional overcurrent relay elements into the model.

$>$ Simulate faults and determine the behavior (time) of overcurrent relay elements.

$>$ Perform a coordination study to determine the relay settings.

\section{Looped System:}

Using the distributed system model from Lab 1, re-save model under new name and make the following alterations outlined in the steps below. 


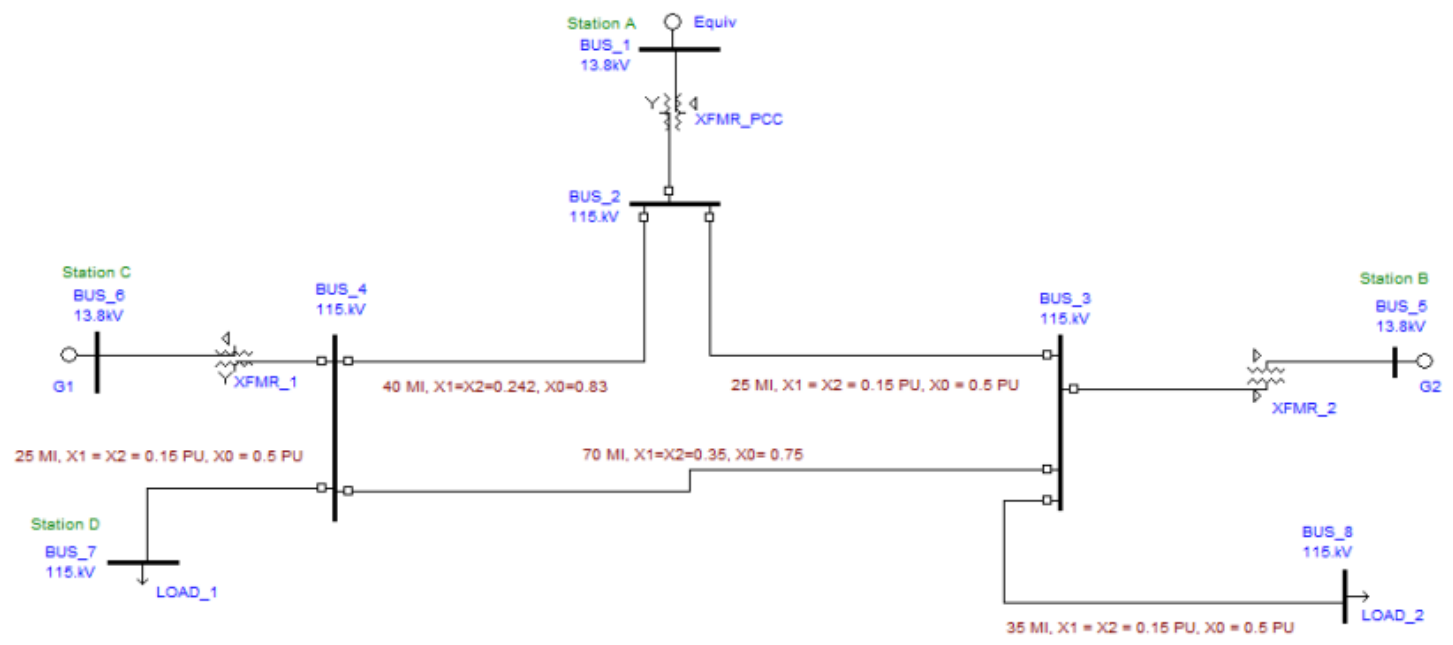

Figure 1: Power system example

\begin{tabular}{|l|r|r|}
\hline Relay & From Bus & To Bus \\
\hline R1 & 2 & 1 \\
R2 & 4 & 6 \\
R3 & 2 & 3 \\
R4 & 2 & 4 \\
R5 & 4 & 7 \\
R6 & 3 & 2 \\
R7 & 3 & 8 \\
R8 & 3 & 4 \\
R9 & 4 & 3 \\
R10 & 4 & 2 \\
R11 & 3 & 5 \\
\hline
\end{tabular}

Table 1: Relay identification for power system in Figure 1

STEP 1. Alter the system from Lab 1, as shown in Figure 1. Alter all values for the Generators, Transformers, and Transmission Lines as follows:

- G_Equiv: $1600 \mathrm{MVA}, 500 \mathrm{kV}, \mathrm{X} 1=\mathrm{X} 2=0.01$ p.u., $\mathrm{X} 0=0.05$ p.u., $\mathrm{PF}=0.98$

- G1: 80 MVA, $115 \mathrm{kV}, \mathrm{X} 1=\mathrm{X} 2=0.16$ p.u., $\mathrm{X} 0=0.1$ p.u., $\mathrm{PF}=0.98$

- G2: 100 MVA, $115 \mathrm{kV}, \mathrm{X} 1=\mathrm{X} 2=0.135$ p.u., $\mathrm{X} 0=0.09$ p.u., $\mathrm{PF}=0.98$

- LOAD_1: $115 \mathrm{kV}, 10 \mathrm{MW}, 1 \mathrm{MVAR}$

- LOAD_2: $115 \mathrm{kV}, 10 \mathrm{MW}, 1 \mathrm{MVAR}$

- XFMR_1: $13.8 / 115 \mathrm{kV}, \mathrm{X} 1=\mathrm{X} 0=0.1$

- XFMR_2: $13.8 / 115 \mathrm{kV}, \mathrm{X} 1=\mathrm{X} 0=0.09$

- XFMR_PCC: $500 / 115 \mathrm{kV}, \mathrm{X} 1=\mathrm{X} 0=0.02$ 
STEP 2. Add (67) phase relays on both ends of each line and on the network side on each transformer using the relay identification numbers indicated in Table 1. Decide which relays must be regular overcurrent, based on their placement in the system, and set in ASPEN. You can start by using the same settings for each relay inside the loop (there will be different default settings for relays outside the loop):

- Relay Type: JBC51

- CT Ratio: $400 / 5=80$

- TAP: 5 A

- Time Dial: 3

- Instantaneous: $100,000 \mathrm{~A}$

- Directional Time Element: (student discretion)

- Directional Instantaneous: unchecked

STEP 3. Neglect the fact that the pickup values should be well above load currents. Assume that relay $\mathrm{R} 1$ has a CTR $=100, \mathrm{TD}=0.5$, and $\mathrm{I} \mathrm{pu}=0.5 \mathrm{~A}$. Assume that relays $\mathrm{R} 5$ and $\mathrm{R} 7$ have a time dial of 1.0 and an $\mathrm{I} \mathrm{pu}=1 \mathrm{~A}$. That relays looking into generator units G1 and G2 are directional with a time dial setting of 0.5 and I $\mathrm{pu}=1 \mathrm{~A}$ with $\mathrm{CTR}=80$. STEP 4 . Perform the necessary simulations and calculations to determine the time dial settings of the relays in the loop. Simulate the faults and adjust the time dial until all relays are properly coordinated as explained in the theory (EE493). Use a coordination interval of 0.4 seconds. Discuss your criteria with the instructor and/or TA. You should use directional units only wherever you think is necessary.

STEP 4. For the loop, first the coordination pairs must be determined. This is done by performing a close-in, 3-phase faults as relays are analyzed first with the necessary simulations for both clockwise and counter clockwise analysis around the loop, starting at the Equivalent source, to determine the time dial settings of the relays in the loop. Use the example from 12.8 in the Blackburn text, referencing Figure 12.7 as your guide for looped coordination.

Simulate the faults and adjust the time dial and current pick-up until all relays are properly coordinated as explained in lecture theory and Chapter 12 (i.e. section 12.8) of the Blackburn text. If necessary, slightly adjust CTR values.

Make an organized presentation with the relay coordination pairs, as shown in Chapter 12 of Blackburn.

Use a loop coordination interval range of 0.3 to 0.4 seconds for the far-bus faults. Reference suggested operating times for close-in faults outlined in section 12.8. Discuss your criteria in your written report. 
**You should use directional units only wherever you think is necessary, and regular OC everywhere else**

\section{Deliverables}

Produce a report with your system screen shot along with the final settings of the relays, and the performance and operational times for each of the faults. Include figures of coordination results. Note that there may be several different solutions to this problem. Discuss how your solution can be improved. 


\title{
A.8 Revised Directional Over-current Relays (67) Instructions
}

\author{
Portland State University \\ Electrical \& Computer Engineering \\ ECE 448/548 Power System Protection I
}

\author{
-Lab 8. Directional Over-current Relays (67) -
}

\section{Introduction}

In this laboratory exercise, the student learns about electromechanical and digital directional over-current relays.

Objectives

At the end of the laboratory exercise the student will be able to do the following:

$>$ Identify the different parts of an electromechanical directional over-current relay

$>$ Determine Time Overcurrent operation of an electromechanical directional element using relay testing equipment and compare results to theoretical calculations for the appropriate relay curve

$>$ Determine the operation zone, based on current angles, of an electromechanical directional element using relay testing equipment

$>$ Implement physical set-up to test SEL-351 using the SEL-RTS (AMS)

$>$ Introduce settings to the relay (Phase and Neutral Ground elements)

$>$ Test the SEL-351 relay with SEL-5401 software and obtain operation times

$>$ Compare results to those found by theoretical calculation

Part I: Testing JBC 51N relay elements

Materials/Equipment

$>$ Avo Pulsar relay tester

$>$ Test leads

$>$ Oscilloscope

$>$ Current probe

$>\mathrm{JBC} 51 \mathrm{~N}$ relay

***The laboratory JBC51N relay manual has generic connections for relay models $\operatorname{JBC51M}(-) Y 1 A$ which do not correspond completely to the configuration of the available JBC51N. To account for this issue, for every figure that references a connection between pins 1 and 11 , use pins 1 and 10 with the same polarity.***

STEP 1: Download JBC51N instruction manual and identify most relevant parts of the relay. Identify the terminals associated with voltage and current source inputs 
as well as relay contacts, which will be used in the Timing Test and Directional Unit Test from the related diagrams. Figure 1 below shows a diagram the connection of three, single phase JBC relays in a power system. Note that the numbers labeled on the connections correspond to physical relay connection pins $1-11$ on this diagram. ***Keep in mind the note above regarding output pin connection number change for pin $11^{* * *}$

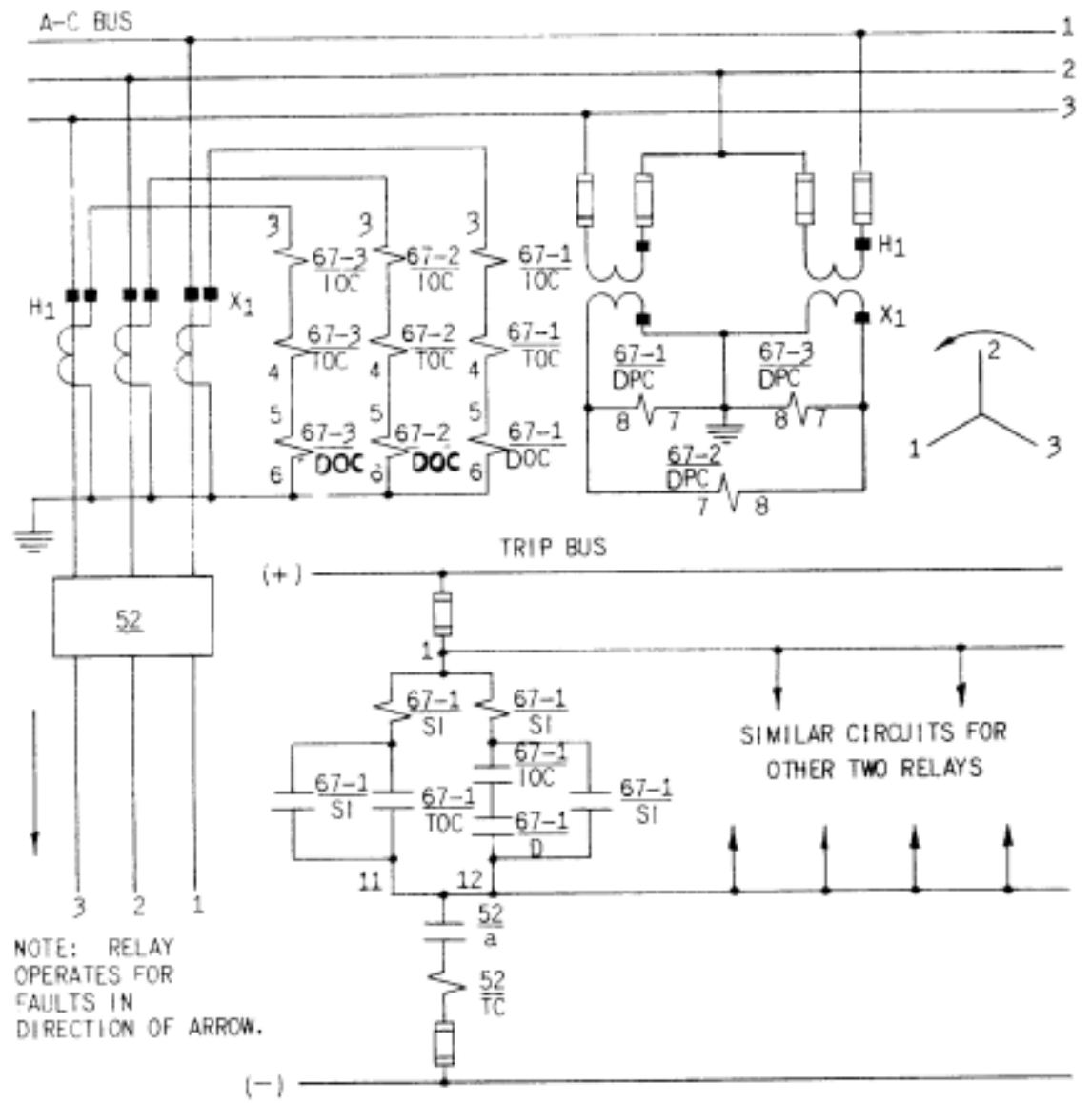

Figure 46: JBC manual relay connections for 3-single phase $\mathrm{JBC}$ relays [Image source: GE JBC51N user manual] 


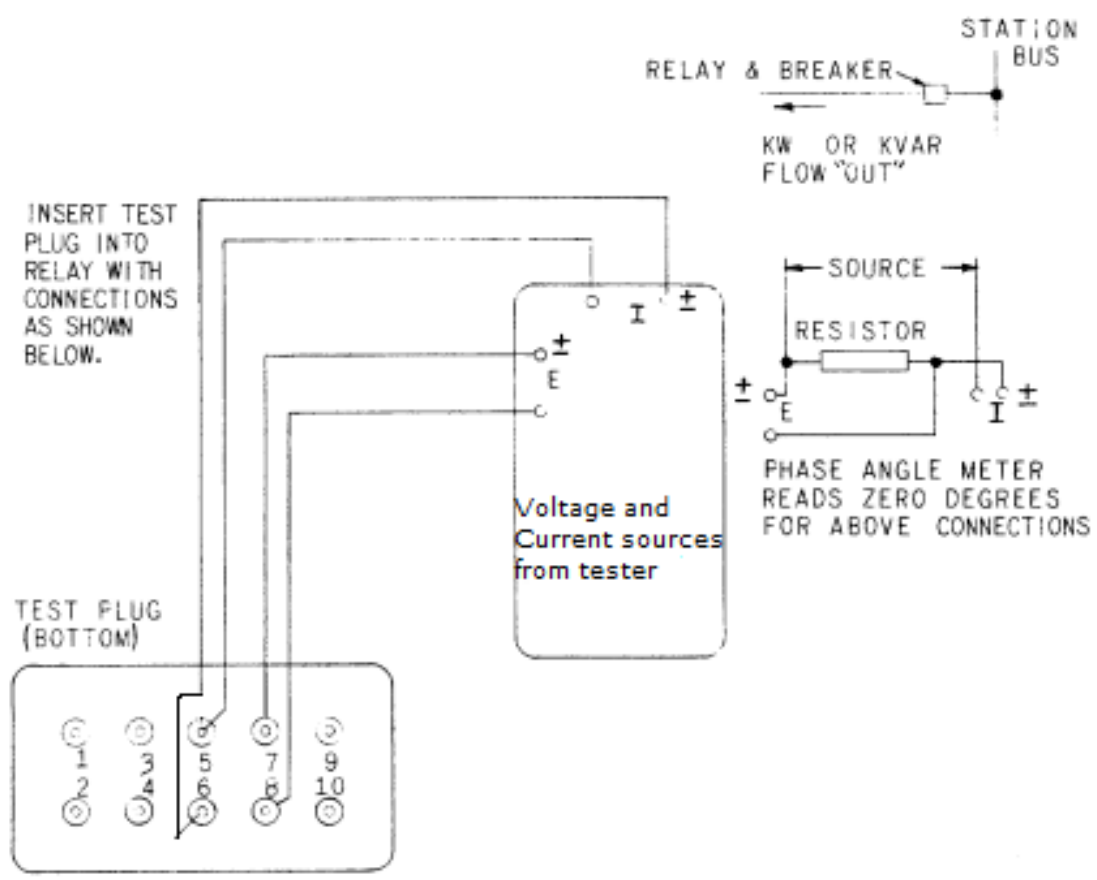

\begin{tabular}{|c|c|c|c|c|c|c|c|c|}
\hline $\begin{array}{l}\text { FOWER FACTOR ANGLE } \\
(D E G . \text { LEAD }]\end{array}$ & $4 \overline{5}$ & $\begin{array}{l}45- \\
90\end{array}$ & $\begin{array}{l}90= \\
135\end{array}$ & $135-$ & $\begin{array}{l}180- \\
225\end{array}$ & $\begin{array}{l}225- \\
270\end{array}$ & $\begin{array}{l}270- \\
315 \\
\end{array}$ & $\begin{array}{l}325- \\
360 \\
\end{array}$ \\
\hline $\begin{array}{l}\text { KW \& KVAR DIRECTIONS } \\
\text { WITH RESPECT TO THE BUS } \\
\end{array}$ & $\begin{array}{l}\text { KN OUTP } \\
\text { KYAR } \\
\text { IX }\end{array}$ & 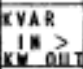 & 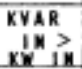 & 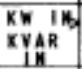 & 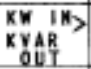 & $\begin{array}{l}\text { KVAR } \\
\text { ouT } \\
\text { oút }\end{array}$ & $\begin{array}{l}\text { KVAR } \\
\text { OUT> } \\
\text { KY QUT }\end{array}$ & $\begin{array}{l}\text { KW OUZ } \\
\text { KYAR } \\
\text { OUT }\end{array}$ \\
\hline $\begin{array}{l}\text { METER READING WITH } \\
\text { PROFER EXT.CONNS. }\end{array}$ & $90-$ & 135 & $\begin{array}{l}180- \\
225\end{array}$ & $\begin{array}{l}225- \\
270\end{array}$ & $\begin{array}{l}270- \\
315\end{array}$ & $\begin{array}{l}315- \\
360\end{array}$ & $\begin{array}{r}0 \\
45\end{array}$ & $\begin{array}{l}45 \\
90\end{array}$ \\
\hline
\end{tabular}

THE ABOVE RANGES OF FHASE ANGLE METER READINGS ARE THE ANGLES BY

WHICH THE CURRENT LEADS THE VOLTAGE WITH THE DESCRIBED CONDITIONS

OF FOWER [KW] AND REACTIVE POWER [KVAR! FLOW WITH THE STATION BUS CONSIDERED AS THE REFERENCE IN ALI CASES. > MEANS GREATER THAN. CAUTION: MAKE CORRECT:ONS FOR METER ERRORS ON LON CURRENTS.

INHERENT IN SOME PHASE-ANGLE METERS.

Fig. 24 (0377A0195-3) Test Connections for Checking Polarity of the External Wiring to the Directional Unit

Figure 47: JBC manual Figure 24, referenced in Pulsar test instructions, for directional unit test input signal connections (modified) [Image source: GE JBC51N user manual]

STEP 2: Download Pulsar manual and reference Table of Contents for relay type. Find relay type. Go to section and read instructions for three of the basic relay testing procedures outlined there, using both the Pulsar manual and the JBC manual as instructed. Figure 3 below shows an excerpt from the Pulsar manual, with notes regarding for testing the directional unit. The Figure 24 referenced in the Pulsar manual is shown above in Figure 2, with modifications, for testing the directional element of the relay. $* * *$ The timing test for the overcurrent element does have different input connections 3 and 4, which are shown in the relay manual. Reference manual, mind the polarities of the connections ${ }^{* * *}$ 


\section{$>$ TIMING TEST \\ $>$ DIRECTIONAL UNIT}

\begin{tabular}{|c|c|c|c|c|c|c|c|}
\hline \multicolumn{7}{|c|}{ PULSAR TEST CONDITIONS } & \\
\hline \multirow[t]{2}{*}{ ASSIGN } & \multicolumn{2}{|c|}{ CONNECT } & \multirow[t]{2}{*}{ TEST } & \multicolumn{2}{|c|}{ VALUES } & \multirow[t]{2}{*}{$\mathrm{HZ}$} & \multirow{6}{*}{$\begin{array}{l}\text { For the laboratory relay } \\
\text { models of JBC51N, } \\
\text { connect } 10 \text { rather than } 11 . \\
\text { There is no } 11 \text { th } \\
\text { connection on these } \\
\text { models. }\end{array}$} \\
\hline & \pm & $\mathrm{N}$ & & & $\angle$ & & \\
\hline $\mathrm{V}_{\mathrm{A}}$ & 7 & 8 & $V_{A}$ & 115 & * & 60.0 & \\
\hline$I_{A}$ & 5 & 6 & $I_{A}$ & 2 & $0^{\circ}$ & 60.0 & \\
\hline MON & 1 & $11 \nless$ & & & & & \\
\hline & & & & & & & \\
\hline
\end{tabular}

\section{DIRECTIONAL UNIT TEST} Set $V_{A}$ and $I_{A}$ for directional unit polarity check based on Figure 24 of $\sqrt{\text { manual }}$ instruction manual and table parameters below. Refer to Phase Angle Relationships Section. All current angles in JBC instruction manual are leading angles, those listed below are lagging angles.

2. Ramp current up or down to determine minimum pickup value.

3. Record the minimum pickup value. Be sure to note which ranges of current angles operate the directional unit. Include this information in your report with a synthesis of how these results compare to the theory of (67) operation based on current flow.

\begin{tabular}{|c|c|c|c|c|c|c|c|c||}
\hline P.F. Angle - Lag & $90-135$ & $135-180$ & $180-225$ & $225-270$ & $270-315$ & $315-360$ & $0-45$ & $45-90$ \\
\hline $\begin{array}{c}\text { Power Flow } \\
\text { KW IN/OUT }\end{array}$ & OUT & $\mathbb{I N}$ & $\mathbb{I N}$ & $\mathbb{I N}$ & $\mathbb{I N}$ & OUT & OUT & OUT \\
\hline \hline
\end{tabular}

4. Contacts should be closed when $\mathrm{KW}$ flow is $\mathrm{IN}$ and open when $\mathrm{KW}$ flow is out.

5. De-initiate $\mathrm{V}_{\mathrm{A}}$ and $\mathrm{I}_{\mathrm{A}}$; VOLTAGE, 1, CURRENT, 1, OFF, EXECUTE.

Figure 48: Pulsar manual testing instructions for the directional unit, with noted adjustments for the laboratory testing process (page 139)

STEP 3: Connect oscilloscope to relay testing circuit.

STEP 4: Perform TIMING TEST on the relay from the Pulsar instruction manual, using the correct input connections from the JBC relay manual. Record results for time operation at the specified fault current of twice the amount of the IPU tap. Note the difference between the Pulsar ammeter and the oscilloscope readings. Compare to calculated operating time values using equations from lecture material.

$$
\mathrm{t}_{\mathrm{r}}=\mathrm{TD} \cdot\left(\frac{5.95}{1-\mathrm{M}^{2}}\right)
$$

Plot calculated and tested results on the provided relay curve from relay manual (Figure 4). 


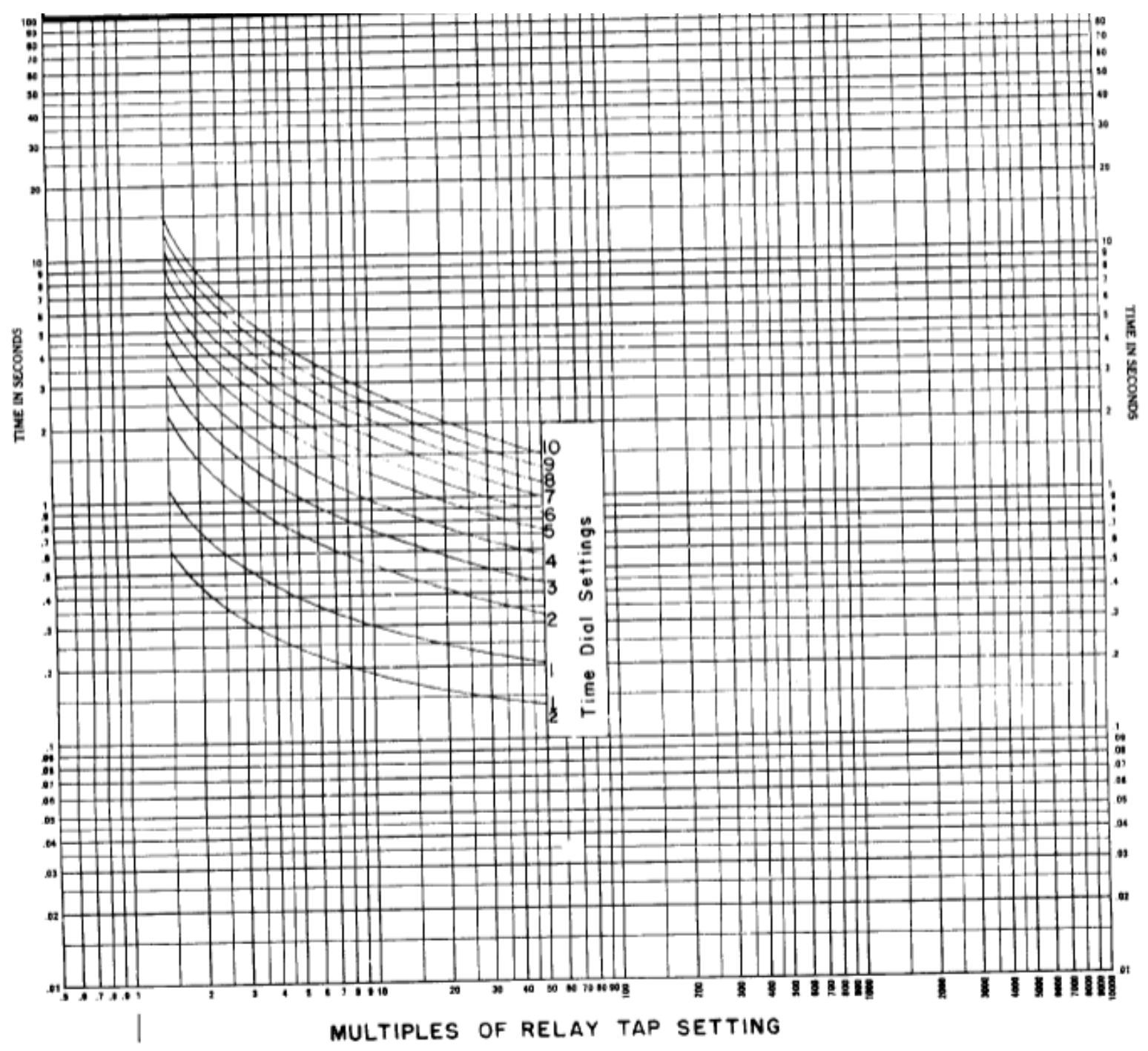

Figure 49: US Inverse Curve U2 from JBC51N instruction manual [Image Source: GE JBC51N user manual]

STEP 5: Perform DIRECTIONAL UNIT test from Pulsar instruction manual using the V and I source input connections shown in Figure 2 above. Record results for IPU value over the range of current angles. Note the difference between the Pulsar ammeter and the oscilloscope readings. Record the results of the minimum IPU value and which range of angles experience directional element operate vs. no operation. Reference Setting Phase Angle Relationships from the Pulsar instruction manual (page 23) for this step.

\begin{tabular}{|c|c|c|c|c|c|c|c|c||}
\hline P.F. Angle - Lag & $90-135$ & $135-180$ & $180-225$ & $225-270$ & $270-315$ & $315-360$ & $0-45$ & $45-90$ \\
\hline $\begin{array}{c}\text { Power Flow } \\
\text { KW IN/OUT }\end{array}$ & OUT & IN & IN & IN & IN & OUT & OUT & OUT \\
\hline \hline
\end{tabular}


Table 1: Current flow direction for lagging current angles [reference Pulsar user manual]

\section{Part II: Introductory procedure to test the SEL-351 relay}

Materials/Equipment
1) SEL-351 Protection System relay
2) SEL-AMS
3) SEL-5401
4) AcSELerator QuickStart software
5) C724 ribbon cable
6) C234A serial port cable
7) C662 USB to serial port cable

STEP 1. Download a copy of the SEL-351 instruction manual. Read the introduction and the section that corresponds to protection elements.

STEP 2. Open AcSELerator Quickset and set the SEL-351 according to the table shown in the next pages pages 2 and 3 of this document).

STEP 3. Connect the AMS to the SEL-351, using the procedure learned in previous laboratory exercises using the appropriate ribbon cable.

STEP 4. Connect the SEL-351 to the computer using the proper cable (C662), and establish communication with the relay using the proper communication settings in the AcSELerator software. Use the knowledge you acquired in the previous digital relay laboratory exercise and the settings listed at the end of this instruction sheet. If the relay password is needed, reference relay instruction manual for Default Passwords. If the C662 cable is unavailable, input setting to relay front panel manually. You will need the C662 cable to input the logic variables prior to testing, as these settings are not programmable through the relay face plate. They are able to be set in the AcSELerator software prior to any communication of AcSELerator to the relay. The relay does not have to be connected for your prep of relay logic settings, so do make a settings case in AcSELerator for your SEL-351 prior to acquiring the $\mathrm{C} 662$ cable.

STEP 5. Connect the AMS testing system to the computer as in previous laboratory exercises with the C234-A serial cable. Open the SEL-5401 program, go to UUT Database and update your SEL-351 relay to be a 5 Amp device. Verify Scale Factors have been adjusted, save and close the UUT Database window and then select the SEL-351 relay model from the menu for the new settings. Before setting the State Values (IA, IB, IC, IN), perform the METER test with the Front Panel command on the tool bar, as done in the previous digital relay laboratory exercise. Verify that the values shown on the relay front panel meter (in primary units) are consistent with the CTR programmed into the SEL-351. If needed, use the SEL-AMS instruction manual as a guide.

STEP 6. If Front Panel METER test results are inaccurate, adjust the Scale Factor of the 
SEL-351 proportionally until values read as expected. Each time the Scale Factor is adjusted, a new case with a new SEL-351 relay must be opened to include this adjusted Scale Factor.

STEP 7. Apply the fault values in the SEL-5401 software as given in Tables 1 - 3 below. Case 1 is a balanced 3- $\varphi$ fault. Cases 2 and 3 correspond with the faults used in Problem 1, Homework 5. From Homework 5, PTR $=(132000 /$ as given

\begin{tabular}{|c|c|c|}
\hline Quantity & $\begin{array}{c}\text { Magnitude } \\
\text { (Primary kV and amps) }\end{array}$ & Angle (degrees) \\
\hline $\mathrm{V}_{\mathrm{A}}$ & 13.2 & 0 \\
\hline $\mathrm{V}_{\mathrm{B}}$ & 13.2 & -120 \\
\hline $\mathrm{V}_{\mathrm{C}}$ & 13.2 & 120 \\
\hline $\mathrm{I}_{\mathrm{A}}$ & 3323 & -90 \\
\hline $\mathrm{I}_{\mathrm{B}}$ & 3323 & 150 \\
\hline $\mathrm{I}_{\mathrm{C}}$ & 3323 & 30 \\
\hline
\end{tabular}

Table 1: Case 1 fault parameters

\begin{tabular}{|c|c|c|}
\hline Quantity & $\begin{array}{c}\text { Magnitude } \\
\text { (Primary kV and amps) }\end{array}$ & Angle (degrees) \\
\hline $\mathrm{V}_{\mathrm{A}}$ & 16 & -6 \\
\hline $\mathrm{V}_{\mathrm{B}}$ & 76 & -120 \\
\hline $\mathrm{V}_{\mathrm{C}}$ & 76 & 120 \\
\hline $\mathrm{I}_{\mathrm{A}}$ & 4389 & -80 \\
\hline $\mathrm{I}_{\mathrm{B}}$ & 40 & -84 \\
\hline $\mathrm{I}_{\mathrm{C}}$ & 40 & -84 \\
\hline
\end{tabular}

Table 2: Case 2 fault parameters

\begin{tabular}{|c|c|c|}
\hline Quantity & $\begin{array}{c}\text { Magnitude } \\
\text { (Primary kV and amps) }\end{array}$ & Angle (degrees) \\
\hline $\mathrm{V}_{\mathrm{A}}$ & 21.4 & -8 \\
\hline $\mathrm{V}_{\mathrm{B}}$ & 76 & -120 \\
\hline $\mathrm{V}_{\mathrm{C}}$ & 76 & 120 \\
\hline $\mathrm{I}_{\mathrm{A}}$ & 1238 & 98 \\
\hline $\mathrm{I}_{\mathrm{B}}$ & 24 & 109 \\
\hline $\mathrm{I}_{\mathrm{C}}$ & 31 & 111 \\
\hline
\end{tabular}

Table 3: Case 3 fault parameters

\section{Deliverables}

Produce a short report with summary of results, including operation time calculations and the hand plotted results on the Time-Current curves, for parts 1 and 2 comparing experimental values to theoretical values. Include the figures of Relay Configuration window, the UUT report results, and the AcSELerator QuickSet settings and a discussion of the tested results compared to the expected results from Homework 5 for Cases A and B. Report is due EOB on Friday, March 22nd. 
In AcSELerator QuickStart, begin by creating New Settings for SEL-351:

SEL-351-6 --> 100 --> $>$ Okay $>$

In the next window, correct settings to correspond w/ $\mathbf{P} / \mathbf{N}$ of relay: $\mathbf{0 3 5 1 6 3 A 3 C 5 4 2 X 1 .}$

Use the following settings to program the SEL-351 for testing:

\begin{tabular}{|c|c|c|c|c|}
\hline Set 1 & $\begin{array}{l}\text { Setting } \\
\text { Symbol }\end{array}$ & Description & Units & Value \\
\hline \multicolumn{5}{|l|}{ General Settings } \\
\hline & CTR & Phase Current Xfmr Ratio & $\mathrm{N} / \mathrm{A}$ & 50 \\
\hline & CTRN & Neutral Phase Current Xfmr Ratio & $\mathrm{N} / \mathrm{A}$ & 50 \\
\hline & PTR & Phase Potential Xfmr Ratio & $\mathrm{N} / \mathrm{A}$ & 1137.47 \\
\hline & PTRS & Sync. Voltage Xfmr Ratio & N/A & 1137.47 \\
\hline & VNOM & Phase PT Nom Volt (L-N) & VSEC & 67 \\
\hline \multicolumn{5}{|l|}{ Line Settings } \\
\hline & Z1MAG & Positive-seq Impedance Mag & $\Omega \sec$ & 1.5 \\
\hline & Z1ANG & Positive-seq Impedance Ang & Degrees & 80 \\
\hline & Z0MAG & Zero-seq Impedance Mag & $\Omega \sec$ & 5.2 \\
\hline & Z0ANG & Zero-seq Impedance Angle & Degrees & 80 \\
\hline & $\mathrm{LL}$ & Line Length & $\%$ & 100 \\
\hline & EFLOC & Fault Locator & $\mathrm{N} / \mathrm{A}$ & $\mathrm{Y}$ \\
\hline \multicolumn{5}{|l|}{$\begin{array}{l}\text { Phase Overcurrent } \\
\text { Elements }\end{array}$} \\
\hline & E50P & Phase Overcurrent Elements & $\mathrm{N} / \mathrm{A}$ & $\mathrm{N}$ \\
\hline \multicolumn{5}{|l|}{$\begin{array}{l}\text { Neutral Ground } \\
\text { Overcurrent Elements }\end{array}$} \\
\hline & $\mathrm{E} 50 \mathrm{~N}$ & Neutral Overcurrent Elements & $\mathrm{N} / \mathrm{A}$ & $\mathrm{N}$ \\
\hline \multicolumn{5}{|l|}{$\begin{array}{l}\text { Residual Ground } \\
\text { Overcurrent Elements }\end{array}$} \\
\hline & $\mathrm{E} 50 \mathrm{~N}$ & $\begin{array}{l}\text { Residual Ground Overcurrent } \\
\text { Elements }\end{array}$ & $\mathrm{N} / \mathrm{A}$ & $\mathrm{N}$ \\
\hline \multicolumn{5}{|l|}{$\begin{array}{l}\text { Negative-Seq } \\
\text { Overrcurrent Elements }\end{array}$} \\
\hline & E50Q & Neg-Seq Overcurrent Elements & N/A & $\mathrm{N}$ \\
\hline \multicolumn{5}{|l|}{$\begin{array}{l}\text { Phase Time- } \\
\text { Overcurrent Elements }\end{array}$} \\
\hline & E51P & Phase Time-Overcurrent Elements & $\mathrm{N} / \mathrm{A}$ & 1 \\
\hline & $51 \mathrm{PP}$ & Phase Time-Overcurrent Elements & A & 4.5 \\
\hline & $51 \mathrm{PC}$ & Curve & $\mathrm{N} / \mathrm{A}$ & $\mathrm{U} 2$ \\
\hline & 51PTD & Time Dial & $\mathrm{N} / \mathrm{A}$ & 2.5 \\
\hline & 51PRS & Electromechanical Reset Delay & $\mathrm{N} / \mathrm{A}$ & $\mathrm{N}$ \\
\hline
\end{tabular}


**ALL OTHER SETTINGS LEAVE AS DEFAULT**

Neutral Ground Time-

Overcurrent

Overcurrent Elements

\begin{tabular}{|c|c|c|c|c|}
\hline & \multirow[t]{5}{*}{ E51N } & $\begin{array}{l}\text { Neutral Ground Time-Overcurrent } \\
\text { Elements Settings }\end{array}$ & N/A & Y \\
\hline & & Neutral Time-Overcurrent Element & A & 0.5 \\
\hline & & Curve & N/A & $\mathrm{U} 2$ \\
\hline & & Time Dial & N/A & 2.5 \\
\hline & & Electromechanical Reset Delay & N/A & $\mathrm{N}$ \\
\hline & \multirow{2}{*}{\multicolumn{2}{|c|}{ **ALL OTHER SETTINGS LEAVE AS DEFAULT** }} & & \\
\hline \multirow[t]{2}{*}{$\begin{array}{l}\text { Residual Ground Time- } \\
\text { Overcurrent Elements }\end{array}$} & & & & \\
\hline & E51G & $\begin{array}{l}\text { Residual Ground Time-Overcurrent } \\
\text { Elements Settings }\end{array}$ & N/A & $\mathrm{N}$ \\
\hline \multirow[t]{2}{*}{$\begin{array}{l}\text { Negative-Sequence } \\
\text { Time-Overcurrent } \\
\text { Elements }\end{array}$} & & & & \\
\hline & E51Q & $\begin{array}{l}\text { Negative-Sequence Time-Overcurrent } \\
\text { Elements Settings }\end{array}$ & N/A & $\mathrm{N}$ \\
\hline \multirow[t]{2}{*}{$\begin{array}{l}\text { Load Encroachment } \\
\text { Element }\end{array}$} & & & & \\
\hline & ELOAD & Load Encroachment Element & N/A & $\mathrm{N}$ \\
\hline \multirow[t]{6}{*}{ Directional Elements } & & & & \\
\hline & E32 & Directional Control Elements Settings & N/A & AUTO \\
\hline & ELOP & Loss-Of-Potential & N/A & $\mathrm{N}$ \\
\hline & DIR 1 - 4 & Level 1 - 4 Direction & N/A & $\mathrm{F}$ \\
\hline & ORDER & Ground Directional Priority & N/A & $\mathrm{V}$ \\
\hline & \multicolumn{3}{|c|}{ **ALL OTHER SETTINGS LEAVE AS DEFAULT** } & \\
\hline
\end{tabular}

\section{Leave all other Set 1 settings as default}

For the Logic 1 variable settings,

$>51 \mathrm{PTC}=1 \ldots$ permanently activate directional control on $51 \mathrm{P}$ elements

$>51 \mathrm{GTC}=1 \ldots$ permanently activate directional control on $51 \mathrm{G}$ elements

$>\mathrm{TR}=51 \mathrm{PT}+51 \mathrm{NT} \ldots \mathrm{TRIP}$ signal just when 51 or $51 \mathrm{~N}$ operate

$>\mathrm{SV} 6=32 \mathrm{PF}+32 \mathrm{QF}$... an internal logic variable which becomes 1 when the positive sequence directional element or the negative sequence directional element indicate forward direction

> Outputs....turn on applicable outputs which will be tested as phases A, B, C, and $\mathrm{N}$.

For the Global 1 settings,

$>\mathrm{LER}=30$

> $\mathrm{PTCONN}=\mathrm{WYE}$ 
Appendix B: Original Assignment Instructions 


\title{
B.1 Original ASPEN Software Introduction Instructions
}

\author{
Portland State University \\ Electrical \& Computer Engineering \\ ECE 448/548 Power System Protection I
}

-Lab 1. ASPEN Software Introduction -

\section{Introduction}

This lab introduces students to the power protection equipment and simulation software that will be used in subsequent laboratory assignments. The laboratory students build a single-source, three-phase radial power circuit model and a three-source, single load looped power circuit model using ASPEN software. The laboratory students review symmetrical components and will analyze the fault results by comparing simulated results to calculated results.

\section{Objectives}

At the end of the laboratory exercise the student will be able to do the following:

- Create simple cases in ASPEN OneLiner, including positive-, negative- and zerosequence impedances of line and generators as well as proper transformer connections.

- Run ASPEN Power Flow on a power system case and perform basic analysis of the results.

- Identify the results of the power flow that have an influence in the fault study

- Perform a basic fault study, obtaining results for three-phase, single line-toground, line-to-line, and line-to-line-to-ground faults in the relevant parts of the system and interpret the results.

\section{PART 1}

\section{Distributed System:}




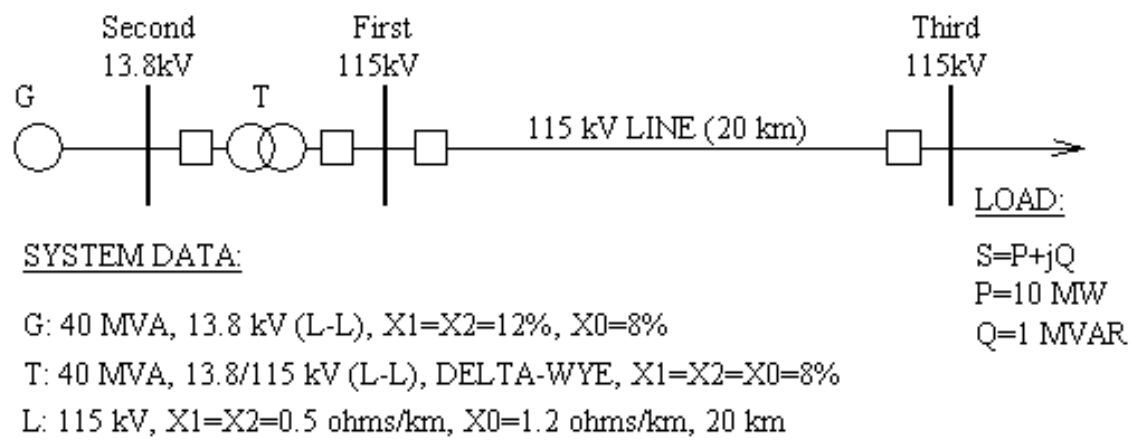

Figure 1: System diagram

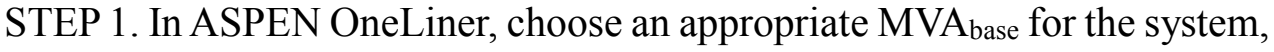
then enter the basic system data in the following order: (Make sure the Device Palette is selected under View)

- $\quad$ FIRST bus $(115 \mathrm{kV})$

- SECOND bus (13.8 kV)

- Enter Cable line information (Neglect susceptance, B, and conductance, G.). This will automatically connect to a third bus $(115 \mathrm{kV})$. Rename this new bus as THIRD.

- Transformer (IMPORTANT: First click the mouse on, or mark, bus FIRST and then mark bus SECOND. The first bus marked cannot contain the DELTA side of the transformer.) Enter the per unit values of the data listed in .

- Add generator to SECOND bus. For transient and subtransient impedance values, use the same values as X1 and X2.

- Add Load information (Must be entered in MW and MVAR. Do not use per unit quantities.)

- Add the Circuit Breakers (Called "RELAY GROUPS" in OneLiner). Do not add relays to the circuit breakers.

*Note: The provided basic system data may need to be adjusted for chosen MVAbase, if MVA base differs from element MVA nominal rating.

STEP 2. Simulate a three-phase fault close to the breaker on the line side of bus FIRST. Include a snap shot of the result of the fault on the one-line diagram for phase A as well as for the sequence component currents. On the Fault Specification window, choose CLOSE-IN FAULT with NO OUTAGE and 3LG. Save the TTY results for the assignment report.

STEP 3. Simulate a single line-to-ground fault at the same point and save the same 
information as requested in Step 2.

STEP 4. Determine the neutral currents for both faults, at the transformer and generator neutral connections.

STEP 5. Determine accuracy of fault calculation by hand or by writing a script in Matlab using theoretical calculations.

**Reference tutorial in section 2-8 of the ASPEN OneLiner on-line help. Use the IEEE09.OLR file in the library for this tutorial if you feel you need practice before beginning the assignment.**

\section{PART 2}

\section{Looped System:}

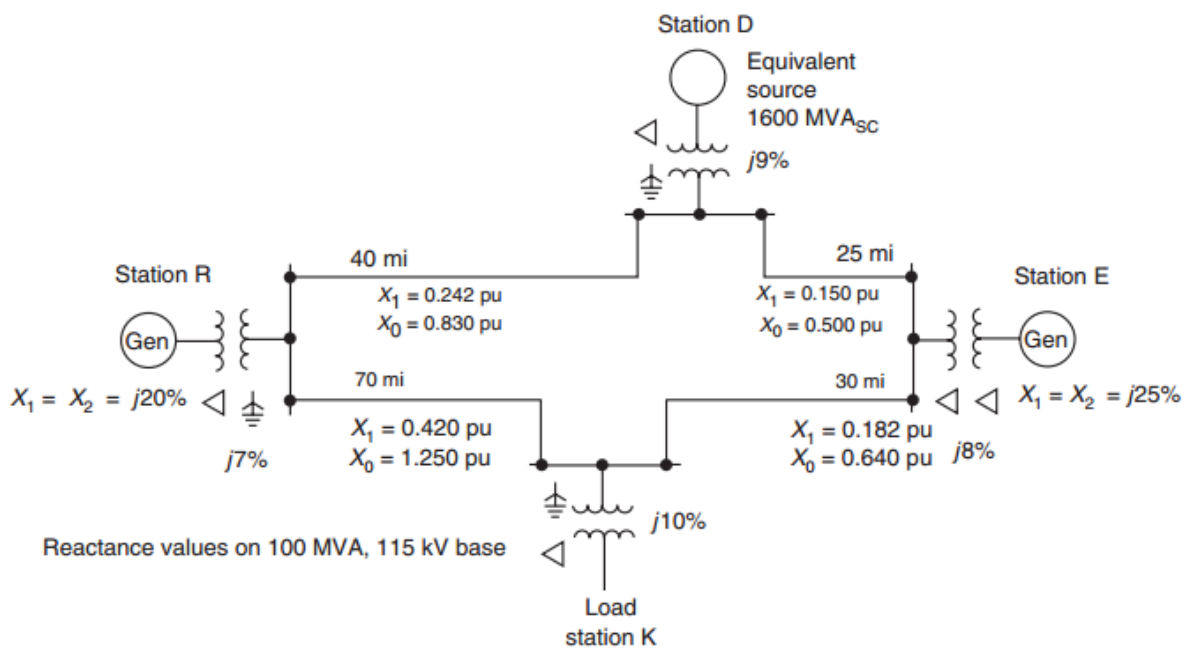

Figure 2: Power system example from Blackburn Relaying Principles (Figure 4.32)

STEP 1. Create the system from Figure 2 using in ASPEN OneLiner, adding circuit breakers to all lines and transformer ends, similar to Part 1 above. Do not add relays to the circuit breakers, this will be done in a future lab exercise.

STEP 2. Simulate three-phase and line-to-ground faults at buses in all four stations and determine the 0-1-2 sequence currents and phase a-b-c currents and voltages at each circuit breaker. 
STEP 3. Add new line between stations $\mathrm{D}$ and $\mathrm{K}$ with parameters of $\mathrm{L}=100 \mathrm{mi}$, $\mathrm{X} 1=0.5 \mathrm{pu}, \mathrm{X} 0=1.5 \mathrm{pu}$.

STEP 4. Repeat the simulations from step 2) with the additional line in the system between stations $\mathrm{D}$ and $\mathrm{K}$.

STEP 5. Simulate line-to-line fault at the midpoint of the new line and determine the sequence $0-1-2$ and phase a-b-c currents and voltages at each end of the new line.

\section{Deliverables}

Print your results in an organized table along with the images of the system one-lines. Compare the results of the calculated v. simulated results of the given faults for the phase currents. Save the models for future exercises. Report is due at EOB on Friday of the second week. 


\title{
B.2 Original Wire Heating and Fuses Instructions
}

\author{
Portland State University \\ Electrical \& Computer Engineering \\ ECE 448/548 Power System Protection I
}

-Lab 2. Wire Heating and Fuses -

\section{Introduction}

This lab introduces the student to the analysis of the dynamic heating of a wire by an electric current. The first-order differential equation will be stated and its solution (temperature vs time curve) will be compared with experimental results. In addition, the student will melt fuse wires and will construct the time-current melting curve for a fuse.

\section{Objectives}

At the end of the laboratory exercise the student will be able to do the following:

- Build the temperature-vs-time heating curve of a wire in free air.

- Compare the results of the experimental $\mathrm{T}(\mathrm{t})$ curve with the predicted by the single model using Matlab or other programming tool and the equation from lecture notes.

- Experimentally build an approximate time-current, I( $(\mathrm{t})$, melting curve for the fuse.

- Verify conductor material type based on thermal behavior.

- Explain the application of a time-current static curve and the dynamic temperature-time response of electrical equipment.

\section{PART 1: Determine the heating characteristics of a metallic wire in free air}

\section{Materials/Equipment}

- Approximately 14 inches of 8 or 10 AWG bare wire

- Watch/clock with second hand

- High current source: MultiAmp SR-51 or SR-76A

- Appropriately rated testing leads

- Multimeter with temperature probe

- Oscilloscope with current probe 


\section{Heating Experiment}

Determine the temperature vs. time curve for the metallic wire for two different currents within the ranges $20 \mathrm{~A}-30 \mathrm{~A}$ and $50 \mathrm{~A}-60 \mathrm{~A}$. Use the simple free-air model to approximate the curves obtained using Matlab.

Cut two pieces of the appropriate length of the bare 8 or 10 gauge wire provided. Attach one wire to a mounting post on fuse holder along with the appropriate testing leads. Attach the oscilloscope current probe to testing leads and prepare oscilloscope to verify measuring the testing current from the MultiAmp ammeter. BE GENTLE, DO NOT SNAP CURRENT PROBES. Prepare your clock by setting to zero.

***Verify set up with a TA before energizing equipment.***

Measure the temperature of the wire through the hole in the glass of the fuse holder. Keep the temperature probe pressed on the wire in order to ensure good contact. Verify the material of the conductor using using standard metal temp vs. time curves.

Energize equipment and raise the current to a point with in the first current range simultaneously starting the clock and noting temperature at $t=0 \mathrm{sec}$. Verify the current input to the wire with the oscilloscope as well as the MultiAmp ammeter. Record the temperature every 15 seconds for the first five minutes. After the first five minutes, temperature can be recorded every 30 seconds.

Repeat this process for the second test at the higher ampere. Plot the results of the tests as well as the theoretical curves in Matlab for comparison of theoretical values to experimental values for the wire material.

\section{PART 2: Determine the melting/clearing time curves of a fuse element}

\section{Materials/Equipment}

- Approximately 14 inches of 20 AWG bare wire

- NEC Copper ampacity table

- Watch/clock with second hand

- Fuse holder

- High current source: MultiAmp SR-51 or SR-76A

- Appropriately rated testing leads

- Oscilloscope with current probe

\section{Heating Experiment}

Determine the melting time vs current curve for the fuse element (log-log) using the simple free-air model to approximate the curves obtained. Verify the material of the fuse element using the time vs current curve and the gauge of the wire. 
Verify the ampacity of the 20 AWG wire at room temperature in free air with the NEC copper ampacity table. Cut the four pieces at appropriate length of the bare 20 gauge wire provided. There will be four separate melting tests done to determine the $I(t)$ curve.

Choose four levels of approximate testing current but do not exceed $60 \mathrm{~A}$, the first level of current being above the rated ampacity for $\mathrm{Cu}$ at room temperature $\left(\sim 75^{\circ} \mathrm{deg}\right)$.

Attach the first wire to mounting posts on fuse holder along with the appropriate testing leads, knowing that the first test will be starting above the rated ampacity for the 20

AWG. Attach the oscilloscope current probe to testing leads and prepare oscilloscope to verify measuring the testing current from the Multi-Amp ammeter. BE GENTLE, DO NOT SNAP CURRENT PROBES. Prepare your clock.

$* * *$ Verify set up with a TA before energizing equipment.***

Starting at the lowest value of your defined range, start you clock and energize the MultiAmp testing unit, applying the first testing approximate testing current. Record this approximate value. Monitor the $\mathrm{Cu}$ wire visually, record the time when the wire turns red hot. This will be the minimum melting time. Record the time that the fuse element breaks. This will be the total melting time.

Repeat this process for the remaining three tests. Plot the results of the tests as well as the theoretical curves for comparison of theoretical values to experimental values for the wire material.

\section{Deliverables}

Produce a short report with plots of the curves and summary of results for parts 1 and 2 comparing experimental values to theoretical values. Report is due EOB on Friday, February 8th. 


\title{
B.3 Original Auxiliary Relay Testing Instructions
}

\author{
Portland State University \\ Electrical \& Computer Engineering \\ ECE 448/548 Power System Protection I
}

\author{
-Lab 3. Auxiliary Relay Testing -
}

\section{Introduction}

In this laboratory exercise the students will study SC and SV type electromechanical auxiliary relays, which are currently used in the power industry and will familiarize students with the Multi-Amp relay testing equipment.

\section{Objectives}

At the end of the laboratory exercise the student will be able to do the following:

- Identify the most relevant parts of the relay testing equipment

- Test electromechanical auxiliary relays: pick-up and operation times

- Be familiar with the features and capabilities of the the electromechanical relay test equipment

\section{Auxiliary Relay Implementation}

In power protection systems, auxiliary relays are used mainly for two general functions: contact multiplication and circuit isolation. In the case of EM relaying as well as control systems there is often a need for more outputs. These outputs will be for a range of functions, including but not limited to multiple tripping, alarms, and operating other equipment, such as recording and data acquisition, and lockout. The auxiliary relays are also applied for contacts that will handle higher currents or voltages in secondary systems along with electrical and magnetic isolation of several secondary circuits. ${ }^{17}$ Due to their simplicity, testing auxiliary relays will allow students to easily become familiar with the Multi-Amp relay testing instruments and learn general concepts of relay testing and operation.

\section{Auxiliary Relay Description}

The auxiliary relays that will be tested in this exercise are SV and SC relays. These relays consist of a single instantaneous current or voltage relay and a number of contacts controlled by the operation of the instantaneous unit. Both the SV and SC use an electromechanical plunger relay, illustrated below in Figure 1. Each type operates in the 
same way, with current or voltage applied to the coil to produce flux, moving the plunger.

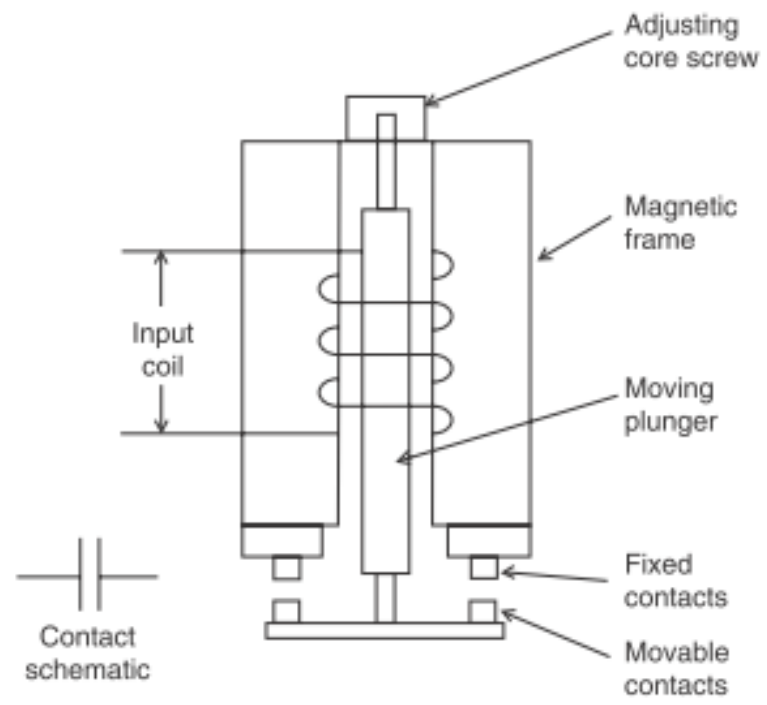

Figure 1: Typical Electromechanical Plunger Relay ${ }^{1}$

\section{PART 1}

\section{Device Identification}

Remove the glass cover and physically inspect the relay, identifying the major working parts of the relays using the relay user manual. Identify the relay contact position.

\section{Testing SV and SC Relays}

\section{Basic Connections}

Figure 2 shows the internal schematic for the SC and SV auxiliary relays. The numbered terminals will be referenced below. Download the appropriate user manuals from D2L.

${ }^{1}$ J. Blackburn, Protective Relaying Principles and Applications, Third Edition. 2006. 


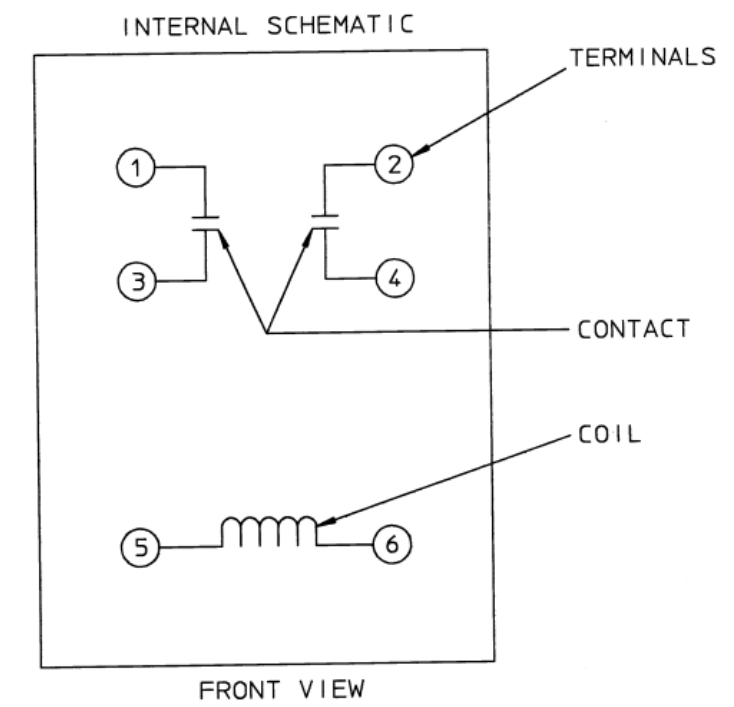

Figure 2: SC/SV internal schematic

Note:

- Use appropriate testing leads for current being applied

- Verify type of voltage source for the SV relay

- Use an external multi-meter to verify results with analog

- Verify MultiAMP outputs are set to zero before energizing

STEP 1. Reference manuals for details regarding proper connection from the Multi-AMP source output to relay operation coil terminals for the SV relays.

$* * *$ for the SC relays, use the current OUTPUT listed for the IAC model relays. The manuals only list testing procedures for the SV relays, while the testing procedure is the same, the source is not***

STEP 2. Reference manuals for details regarding proper connection to relay contact terminals.

STEP 3. Consult a TA to confirm proper testing connections before continuing.

\section{Pick Up Value Verification}

This first procedure will simply verify the value that the relay operates at. Both the SC and SV relays have the same schematic and are identical in operation with the exception that the SC operates on current and the SV operates on voltage.

STEP 1. Verify pick-up value marked on core screw. Adjust core screw to different pick-up value if desired.

STEP 2. Verify that the connections are as described in the MultiAMP user manual and have been reviewed by a TA. Verify that the MAIN 
CONTROL and AUX CONTROL are dialed down to zero.

STEP 3. Follow manual instructions for testing Pick Up value verification. Verify tester settings for Pick-Up verification test with a TA before proceeding to STEP 4.

STEP 4. Energize testing unit. Push the INITIATE button, and begin to adjust the output knob slowly, watching the device meters. Once the continuity light begins to flicker, switch to adjusting the AUX CONTROL until the relay activates. Record this value and compare with value set on relay.

\section{Operation Timing}

Using the same connections and settings the relays will be tested for operation time at a number of different test instrument outputs. This procedure is not an extremely practical test, but serves to familiarize the student with the timing features of the Multi-Amp test instrument.

STEP 1. Verify that the connections and settings of the relay are identical to those used in the pick-up verification.

STEP 2. The timer controls for the relay testers are different, based on model type.

SR-51 series:

a) Make sure tester is de-energized and that relay operation coil contacts are disconnected.

b) Set TIMER OPERATION SELECTOR dial to the appropriate contact positions (NO or NC) to MOM and the toggle to CONT.

c) Based on the set pick up value of the relay, choose a target tripping value slightly higher than the pick-up value.

d) Energize the unit and hold the INITIATE button as the current is ramped up to the target value. Using an external digital multi-meter may be preferred. Once the target value is reached, release INITIATE. The value has been set.

e) Set the TIMER OPERATION SELECTOR to MAINT and the toggle to TIMER.

f) Energize tester, verify clock is set to zero and that the desired units for the timer are selected. Push INITIATE button very briefly and record operation time clocked by relay. Convert time into seconds if operating in cycles.

g) Repeat twice. Document results.

SR-76 series:

a) Make sure tester is de-energized and that relay operation coil contacts are disconnected.

b) Set INITIATE CONTROL dial to the appropriate contact positions (NO or 
NC) to MOM and the toggle to TIMER

c) Based on the set pick up value of the relay, choose a target tripping value slightly higher than the pick-up value.

d) Energize the unit and hold the INITIATE button as the current is ramped up to the target value. Using an external digital multi-meter may be preferred. Once the target value is reached, release INITIATE. The value has been set.

e) Set the INITIATE CONTROL to MAINT and the toggle to TIMER.

f) Energize tester, verify clock is set to zero and that the desired units for the timer are selected. Push INITIATE button very briefly and record operation time clocked by relay. Convert time into seconds if operating in cycles.

g) Repeat twice. Document results.

\section{Deliverables}

Produce a short report with summary of results of relay tests for pick-up value verification and operation time. Report is due by the beginning of lab the following week. Electronic submissions are welcome. 


\title{
B.4 Original Current Transformer Testing Instructions
}

\author{
Portland State University \\ Electrical \& Computer Engineering \\ ECE 448/548 Power System Protection I \\ -Lab 4. Current Transformer Testing-
}

\section{Introduction}

In this laboratory exercise the students will be introduced to CT operation and testing.

Objectives

At the end of the laboratory exercise the student will be able to do the following:

- Determine CT accuracy class and burden rating

- Construct the magnetization curves of different CT ratios

- Experimentally determine CT burden through magnetization curve analysis

- Compare the experimental results with the burden rating given by the manufacture in the CT datasheet.

\section{RT 1: Excitation Test}

\section{Materials/Equipment}

- Veris CT data sheet for CT models AL500 and AL101

- Veris CT models AL500 and AL101

- Multi-Amp test unit manual

- MultiAmp test unit

- Oscilloscope

- Current probe

- Voltage Probe 


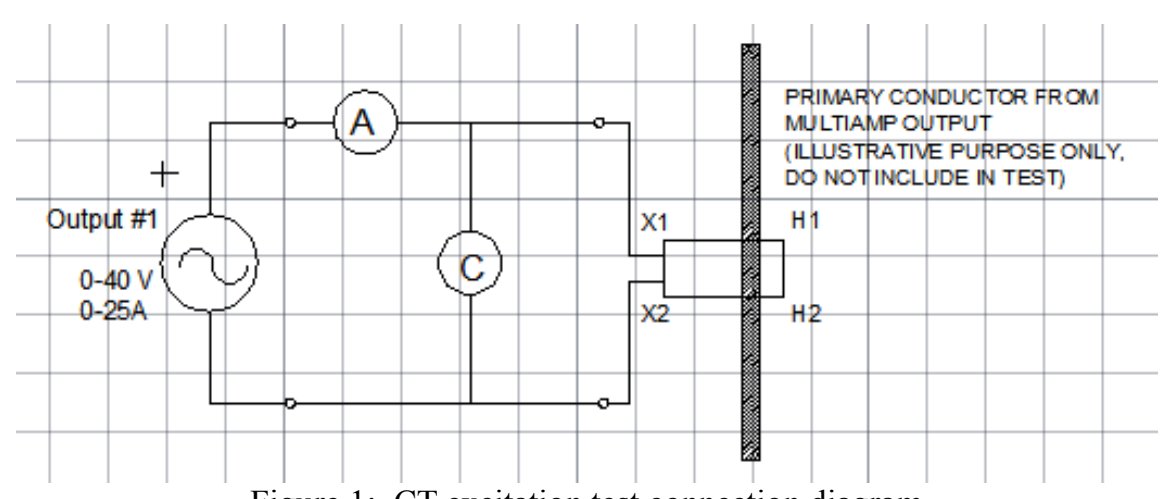

Figure 1: CT excitation test connection diagram

\section{Excitation Test}

For both CT models listed run the following test: ${ }^{18}$

- Refer to CT data sheets and course resources to determine CT accuracy class and rated burden (covert to Ohms).

- Set oscilloscope to read voltage and current.

- Connect the secondary CT leads to the $25 \mathrm{~A}$ output with the oscilloscope voltage probe and current probe attached, as shown in Figure 1.

- Record the test voltages at intervals of approximately $0.1 \mathrm{~V}$ over a range of voltages from approximately zero up to the voltage point where the secondary current is measured at approximately $250 \%$ of rated secondary current.

- ${ }^{* *}$ Never decrease the voltage during this test. If a lower voltage value needs to be tested, the test must begin from zero.**

- Slowly decrease the voltage to zero to demagnetize the CT. Improper deenergization will lead to continued saturation of the CT core.

- ** Note: Saturation can be reversed by re-energizing the CT back to saturation and then slowly decreasing the voltage to zero**

- Create a log-log plot of the data for both CT models. Observe the difference in the curves based on difference in CT ratio. Compare the results of the experimental data to the results of the manufacturer's data sheets by examining the experimental curve and calculating the approximate CT burden to the rated burden.

\section{PART 2: Overcurrent and burden tests}

\section{Materials/Equipment}

- Veris CT data sheet for CT model AL500

- Veris CT model AL500

${ }^{18}$ Excitation test procedure adapted from Back to Basics - Current Transformer Testing, C.W. Valence, http://www.netaworld.org/files/neta-journals/NWfa04-WerstiukPart1.pdf 
- Rheostat bank

- Multi-Amp test unit manual

- MultiAmp test unit

- Oscilloscope with two current probes

Over-current and burden test procedure

The following test will be performed on the AL500 model CT only.

a) Turn on oscilloscope. Set oscilloscope to read RMS current for both current probes. Verify correct range has been set for the current probe and oscilloscope.

b) Connect the secondary leads of the AL500 to the rheostat bank.

c) Connect the XX AWG leads to the 0-100 A output, and appropriate ground, on the MultiAmp test unit, making sure to thread the CT through these connections. These connections serve as the primary conductor on the CT.

d) From the oscilloscope, connect one current probe around the primary conductor and one current probe around the secondary lead.

e) Verify that the control knob of the MultiAm test unit is at zero position.

f) Verify connections with a TA before proceeding

g) With TA approval, energize MultiAmp and slowly increase current output, monitoring current measurements of both sides of transformer. Verify the turns ratio experimentally under no burden.

h) Record results with screen shot of waveforms.

i) Slowly decrease the voltage to zero to demagnetize the CT. Improper deenergization will lead to continued saturation of the CT core.

j) Repeat steps 7 through 9 for both rated burden and rated over burden.

k) Finally, under rated burden, increase the current 120 -140\% over primary current rating and record result of current waveform along with current values for both primary and secondary sides of the transformer.

\section{**Do not leave MultiAmp tester energized at high currents for long. Be timely in your record taking to avoid equipment overload.**}

\section{Deliverables}

Produce a short report with the log-log plots of experimental data. Briefly discuss the results of the core saturation for both turns ratios. Include the screen shots of overcurrent and burden tests as well as their corresponding current measurements on the primary and secondary sides of the CT. Briefly discuss results of the over-current and overburden tests and their effect on transformer core saturation. Report is due EOB on Friday, February 15 th. 


\title{
B.5 Original EM Relay Radial Over-current Protection (50/51) Instructions
}

\author{
Portland State University \\ Electrical \& Computer Engineering \\ ECE 448/548 Power System Protection I
}

-Lab 5. EM Relay Radial Over-current Protection (50/51)-

\section{Introduction}

In this laboratory exercise the students will be introduced to common electromechanical over-current relays currently used by the power industry.

\section{Objectives}

At the end of the laboratory exercise the student will be able to do the following:

- Identify the most important part of an electromechanical over-current relay

- Explain the application of each part of the relay

- Determine the pick-up current of the inverse-time relay element using a relay testing equipment

- Construct the time-current curve of the EM relay

- Determine the instantaneous current of the inverse-time relay element using a relay testing equipment

- Compare the results with the characteristics given by the manufacture in the relay's manual

\section{Materials/Equipment}

- MultiAmp relay tester

- Test leads

- Oscilloscope

- Current probe

- IAC 53B

- IAC 77B

- Relay curves for both relay types 

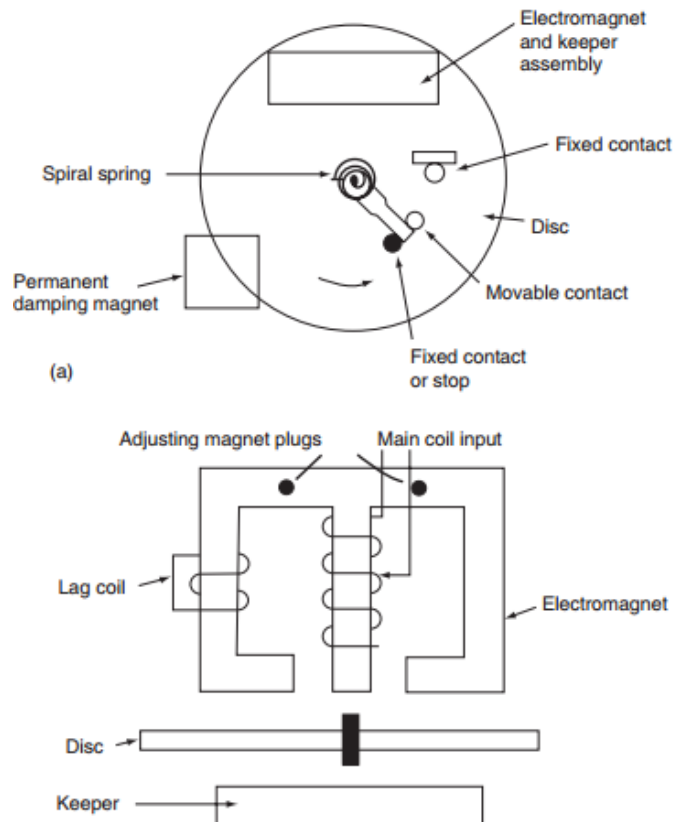

Figure 1: Induction disk construction schematic [Image source: Blackburn text]

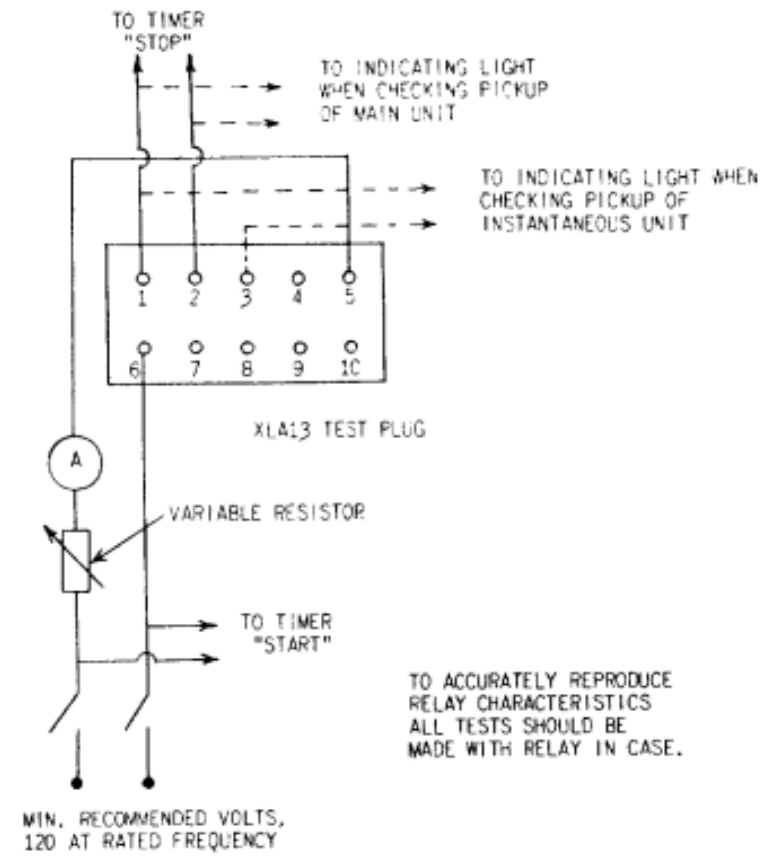

Figure 2: IAC relay test connections for pick-up and time curve settings [Image source: GE IAC user manual] 


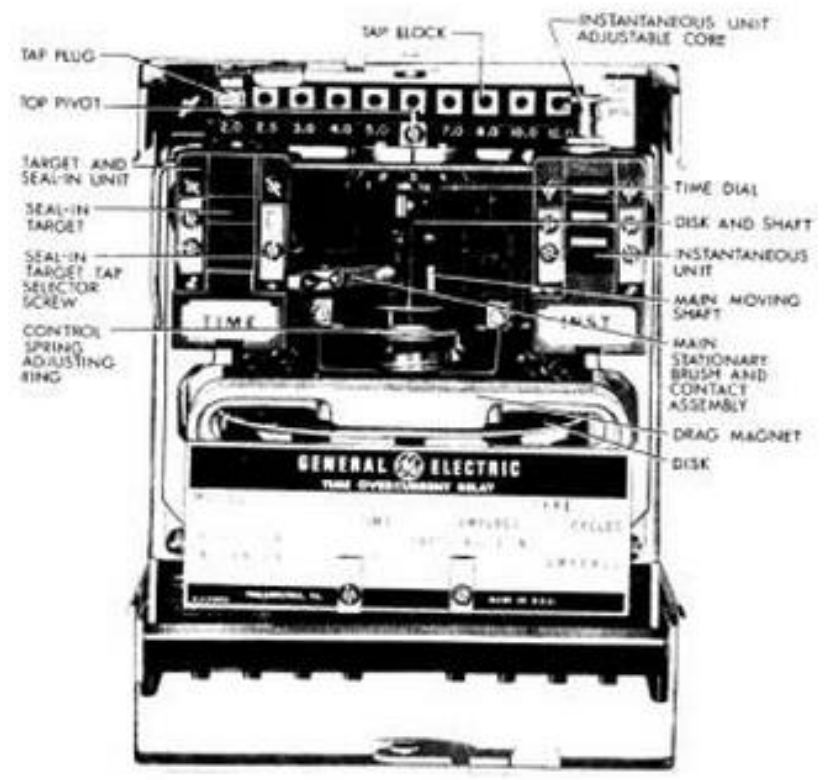

Figure 3: IAC relay elevation description [Image source: GE IAC user manual]

\section{Basic Connections}

Figure 2 shows the internal schematic of the IAC relays. The numbered terminals in the schematic will be referenced below:

- Connect a current source from the MultiAmp unit to relay terminals 5 and 6.

- Connect the Relay Contact posts to relay terminals 1 and 2 for time delay.

- Connect relay terminals 1 and 3 for the instantaneous element.

\section{PART 1: Testing IAC 53B relay elements (Very Inverse)}

STEP 1: Download IAC53B manual and identify most relevant parts of the relay

STEP 2: Download MultiAmp manual.

STEP 2: Reference MultiAmp manual Table of Contents for relay type. Find relay type. Go to section and read instructions for basic relay testing procedure outlined there.

STEP 3: Connect oscilloscope to relay testing circuit.

STEP 3: Perform Pick-up Test on the relay. Record results and compare to settings. 
Note the difference between the MultiAmp ammeter and the oscilloscope readings.

STEP 4: Perform Time Current Characteristic test. Record results for time and current. Note the difference between the MultiAmp ammeter and the oscilloscope readings. Compare to calculated operating time values using equations from lecture material. Plot both results on provided relay curve from relay manual.

STEP 5: Perform Instantaneous Operation test. Record result and compare to physical settings.

\section{PART 2: Testing IAC 77B relay elements (Extremely Inverse)}

STEP 1: Download IAC77B manual and identify most relevant parts of the relay

STEP 2: Download MultiAmp manual.

STEP 2: Reference MultiAmp manual Table of Contents for relay type. Find relay type. Go to section and read instructions for basic relay testing procedure outlined there.

STEP 3: Connect oscilloscope to relay testing circuit.

STEP 3: Perform Pick-up Test on the relay. Record results and compare to settings. Note the difference between the MultiAmp ammeter and the oscilloscope readings.

STEP 4: Perform Time Current Characteristic test. Record results for time and current. Note the difference between the MultiAmp ammeter and the oscilloscope readings. Compare to calculated operating time values using equations from lecture material. Plot both results on provided relay curve from relay manual.

\section{Deliverables}

Produce a short report with summary of results, including operation time calculations and the hand plotted results on the Time-Current curves, for parts 1 and 2 comparing experimental values to theoretical values. Report is due EOB on Friday, February 22nd. 


\title{
B.6 Original Coordination of OC Relays in Radial Systems Instructions
}

\author{
Portland State University \\ Electrical \& Computer Engineering \\ ECE 448/548 Power System Protection I
}

\begin{abstract}
-Lab 6. Coordination of OC Relays in Radial Systems-
Introduction

In this laboratory exercise the students will be introduced to the coordination of overcurrent relays in a radial power system using an industry software program (ASPEN OneLiner).
\end{abstract}

\section{Objectives}

At the end of the laboratory exercise the student will be able to do the following:

- Create a radial case in ASPEN OneLiner containing the required information to do fault studies and overcurrent relay coordination

- Introduce data of phase and ground overcurrent relay elements into the model

- Simulate faults and determine the behavior (time) of overcurrent relay elements

- Perform a coordination study to determine the relay settings

\section{Distributed System:}

Using the distributed system model from Lab 1, re-save model under new name and make the following alterations:

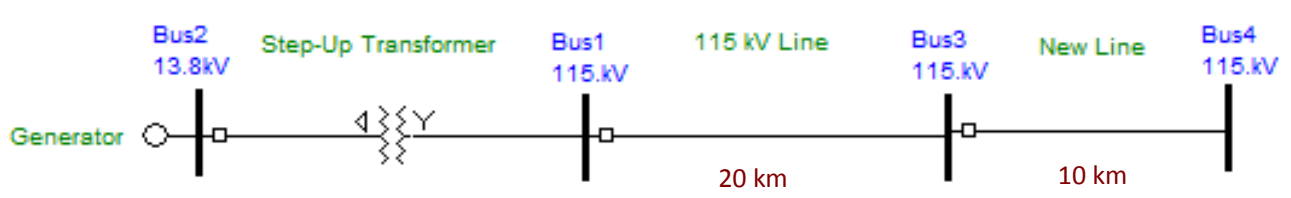

System Data:

G: 40 MVA, $13.8 \mathrm{kV}$ (L-L), X1=X2=12\%, X0 = 8\%

T: 40 MVA, $13.8 / 115 \mathrm{kV}$ (L-L, Dy11), X1 $=\mathrm{X} 2=\mathrm{X} 0=0.2$ (for 100 MVA system base)

$\mathrm{L}: 115 \mathrm{kV}, \mathrm{X} 1=\mathrm{X} 2=0.5 \mathrm{ohms} / \mathrm{km}, \mathrm{X} 0=1.2 \mathrm{ohms} / \mathrm{km}$ (adjust values to $Z_{\mathrm{pu}}$ )

Figure 1: ASPEN radial system model 
STEP 1. Simulate a 3LG fault at the same point as done in Lab 1, near Bus1 on the 20 $\mathrm{km}$ line side, to affirm the results found in lab 1 are still the same. Record fault current values of simulation with a figure of the model. Verify these results with TAs to make sure your system model is correct.

STEP 2. Delete the load on the Bus 3 and add new $10 \mathrm{~km}, 115 \mathrm{kV}$ line between Bus3 (program will automatically create the new Bus 4) with the following impedances:
8) $\mathrm{Z}_{\mathrm{L} 1}=\mathrm{Z}_{\mathrm{L} 2}=\mathrm{j} 0.1$ p.u.
9) $Z_{\mathrm{L} 0}=j 0.3$ p.u.

STEP 3. Insert a circuit breaker located at Bus3 on the $10 \mathrm{~km}$ line side, as shown in Figure 1, using the Relay $\rightarrow$ New Relay Group.

STEP 4. Insert a group of phase relays in the circuit breaker located at Bus3 on the $10 \mathrm{~km}$ line side by selecting the circuit breaker and choosing the OC Phase Relay under Properties $\rightarrow$ Add (highlight CB and right click). Set the following characteristics for the relay:

ID:

Relay Type:

CT Ratio:

Ipu:

Time Dial:

Instantaneous:
R1

\section{General Electric IAC53}

$400 / 5=80$

5 Amp

3

STEP 5. Insert a group of phase relays in the circuit breaker located at Bus 1 of the 20 $\mathrm{km}$ line side:

ID:

R2

Relay Type:

General Electric IAC53

CT Ratio:

Ipu:

$600 / 5=120$

Time Dial:

5 Amp

Instantaneous:

100,000 Amps 
STEP 6. Again, using the same procedures as in Lab 1, simulate a three phase fault close to the circuit breaker located close to Bus3 on the $10 \mathrm{~km}$ line side. Examine and record the fault currents on the one-line diagram with a figure.

STEP 7. Using the same procedure that produced the fault currents on the one-line diagram, display all relay operation times by choosing the clock icon on the program toolbar. Examine and record the relay operation times on the one-line diagram with a figure. Analyze results and determine if the relay operation sequence is correct. Compare analysis determination with relay curves.

STEP 8. Choosing Relay Curves in the Relay menu, display the relay curves at the marked relay group (circuit breaker). Use the Add command in the Relay Curves window to Add Relay Curves and add the relay from the other relay group. Under the Show menu choose Relay Operations for 1 Fault. Record the relay curves for this fault with a figure. Verify coordination with the curves, adjusting settings for proper coordination, if necessary.

STEP 9. Add another IAC53 relay on the $13.8 \mathrm{kV}$ side of the transformer near Bus2. Calculate the $\mathrm{CT}$ rating using $\mathrm{I}_{\mathrm{sc}}$ of Bus 2 provided in Table 1 above and choose appropriate rated CTR for the phase relay. Choose $\mathrm{I}_{\mathrm{pu}}=5 \mathrm{~A}$ for this new relay and calculate the time dial settings for a proper coordination with the rest of the system and put your systems in the ASPEN relay data window. Plot the relay and the transformer damage curve together with the curves of the rest of the relays. Verify coordination graphically with these curves. Write your conclusion with regard to coordination result.

\section{Deliverables}

Produce a report with an analysis of the relay coordination, including any coordination issues and solution results as well as the figures of system results and relay curves detailed in steps 1 through 9 . Report is due by email at the EOB on Friday, March $1^{\text {st }}$. 


\title{
B.7 Original Digital Relay Radial Over-current Protection (50/51) Instructions
}

\author{
Portland State University \\ Electrical \& Computer Engineering \\ ECE 448/548 Power System Protection I
}

\author{
-Lab 7. Digital Relay Radial Over-current Protection (50/51)- \\ Introduction
}

In this laboratory exercise the student will be introduced to the SEL-551 relay. It will be done using the SEL-RTS relay testing equipment.

Objectives

At the end of the laboratory exercise the student will be able to do the following:

- Implement physical set-up to test SEL-551 using the SEL-RTS (AMS)

- Communicate with the relay using the SEL-AMS

- Introduce settings to the relay (Phase and ground elements)

- Properly set a test using the SEL-5410 software

- Test the relay with the AMS and obtain operation times

- Verify operation results by comparing with results found under software simulation

Materials/Equipment

- SEL-AMS

- SEL-5401

- AcSELerator QuickStart software

- SEL-551

- C750A ribbon cable

- CXXX RS-485 cable

- C734A serial cable 


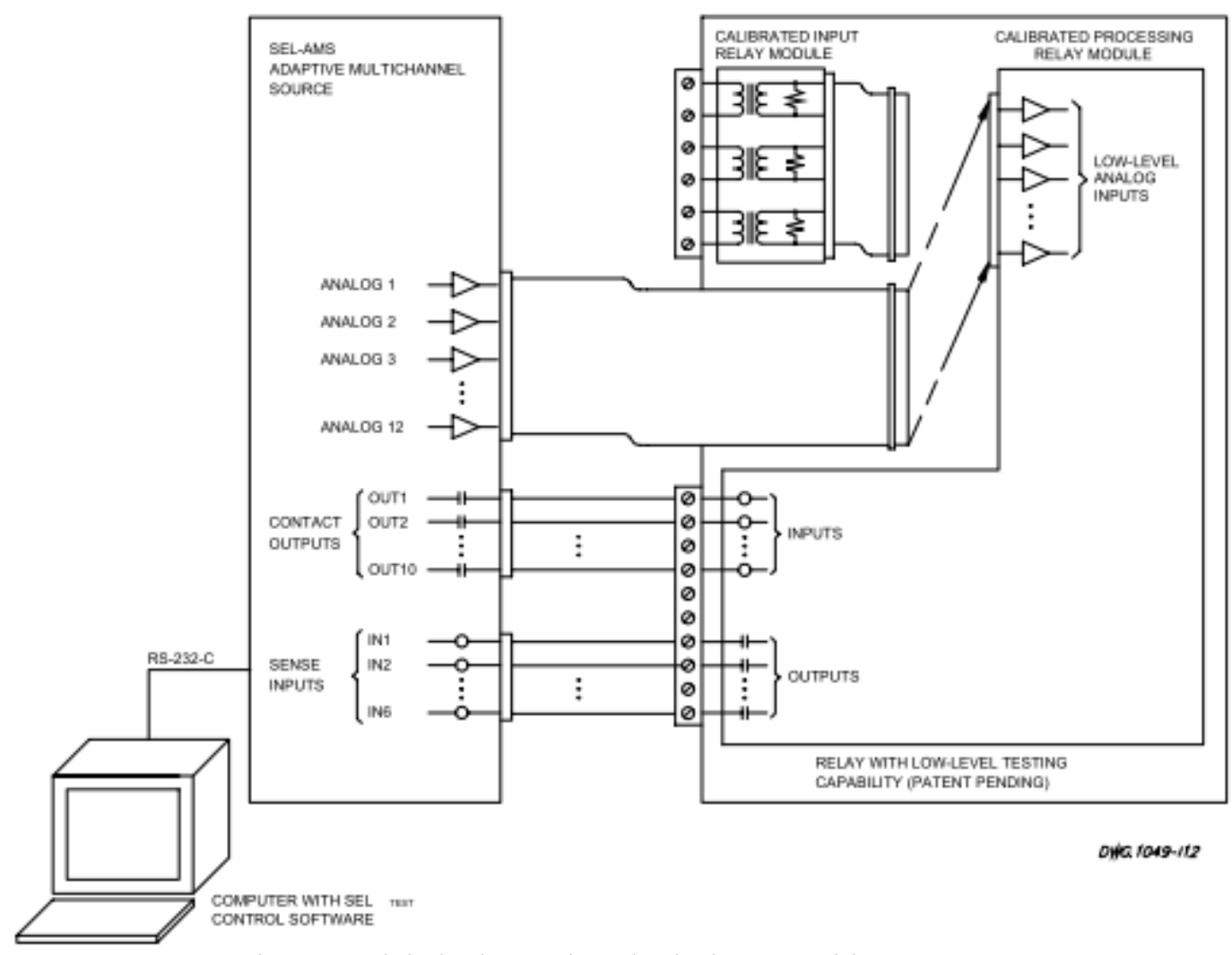

Figure 1: Digital relay testing physical set-up with SEL-AMS

[Image source: SEL-AMS user manual]

\section{Instructions}

STEP 1: Open the Instruction Manuals for the SEL-AMS and SEL-551. Verify that the electrical connections from the SEL-AMS to the SEL-551 are correct using the wiring diagram available in the manual of the SEL-AMS.

STEP 2: To verify SEL-AMS is handshaking with PC, in the SEL-5401 software, go to File --> Open --> C:// --> Program files (x86) --> SEL --> SEL-5401 --> Data --> SAMPLE.RTA, open this RTA file. Download and run this test. When red light on Contact Output Status 1 turns red and the SEL - 5401 window Sense Input shows a $67 \mathrm{sec}$ trip, you know you have a communication between devices.

STEP 3: File --> New Relay Config window appears, go to New relay, UUT Database window appears, choose Relay Type --> SEL-551. In the UUT File Selection box, choose 1 Amp relay file and Apply.

Back to Relay Config window, go to $\mathbf{1 4 . 0 0}$, if so choose --> Okay Begin setting States up in the Standard tab defining phase current values as 1004 /CTR A (the same as close-in fault on Bus1 in Lab 6). Contact Outputs 
for OUT1 and OUT2 set to ('c'). Set INPUTS --> IN1 to $\mathbf{C}->\mathbf{O}$.

$* *$ Record Relay Config window settings as a figure for your report

STEP 4: Download and run the program on the relay. Open UUT report to view and record results for the lab report.

\section{Deliverables}

A written lab report is due complete with figures of Relay Configuration window, the UUT report results, and the printed page of relay settings from the pre-lab exercise. A discussion of the tested results compared to the expected results characterized by the operation time of relay R2 in the ASPEN simulated model from Lab 6 is also required for this written report. The report is due EOB on Friday, March 8, 2013. 


\title{
B.8 Original Protection Coordination of Looped Systems Instructions
}

\author{
Portland State University \\ Electrical \& Computer Engineering \\ ECE 448/548 Power System Protection I
}

\section{-Lab 8. Protection Coordination of Looped Systems-}

\section{Introduction}

In this laboratory exercise the students will be introduced to the coordination of overcurrent relays in a looped power system using a industry software program (ASPEN OneLiner).

\section{Objectives}

At the end of the laboratory exercise the student will be able to do the following:

- Create a looped case in ASPEN OneLiner containing the required information to perform fault studies and overcurrent relay coordination.

- Introduce data of phase and ground directional overcurrent relay elements into the model.

- Simulate faults and determine the behavior (time) of overcurrent relay elements.

- Perform a coordination study to determine the relay settings.

\section{Looped System:}

Using the distributed system model from Lab 1, re-save model under new name and make the following alterations outlined in the steps below.

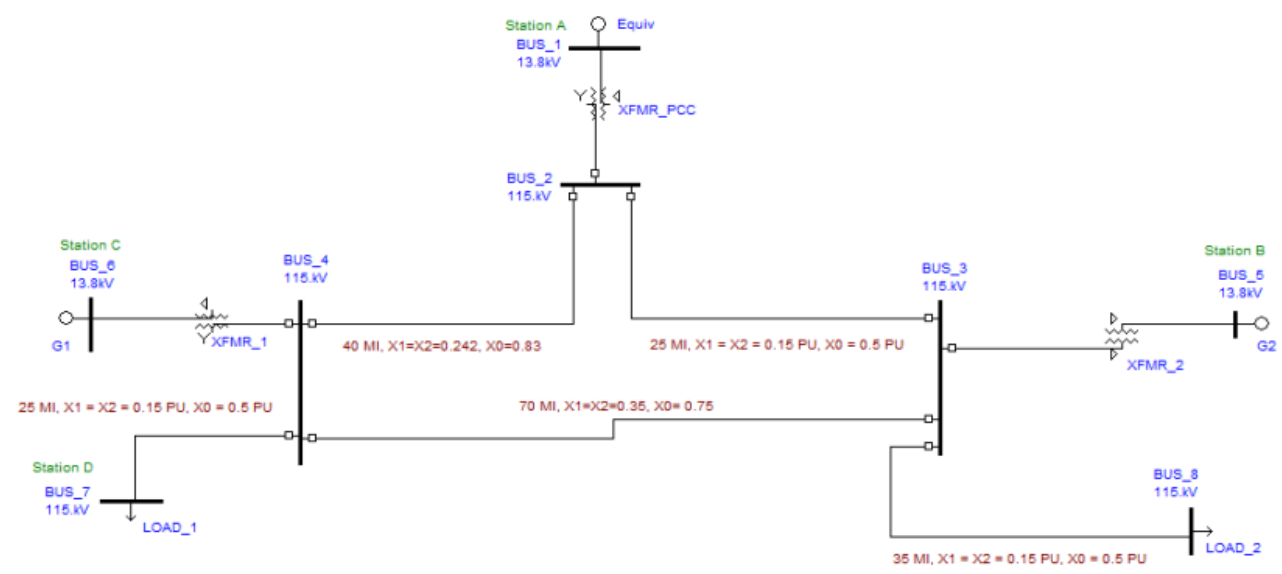

Figure 1: Power system example 


\begin{tabular}{|l|r|r|}
\hline Relay & From Bus & \multicolumn{1}{|c|}{ To Bus } \\
\hline R1 & 2 & 1 \\
R2 & 4 & 6 \\
R3 & 2 & 3 \\
R4 & 2 & 4 \\
R5 & 4 & 7 \\
R6 & 3 & 2 \\
R7 & 3 & 8 \\
R8 & 3 & 4 \\
R9 & 4 & 3 \\
R10 & 4 & 2 \\
R11 & 3 & 5 \\
\hline
\end{tabular}

Table 1: Relay identification for power system in Figure 1

STEP 1. Alter the system from Lab 1, as shown in Figure 1. Alter all values for the Generators, Transformers, and Transmission Lines as follows:

- G_Equiv: $1600 \mathrm{MVA}, 500 \mathrm{kV}, \mathrm{X} 1=\mathrm{X} 2=0.01$ p.u., $\mathrm{X} 0=0.05$ p.u., $\mathrm{PF}=0.98$

- G1: 80 MVA, $115 \mathrm{kV}, \mathrm{X} 1=\mathrm{X} 2=0.16$ p.u., $\mathrm{X} 0=0.1$ p.u., $\mathrm{PF}=0.98$

- $\quad$ G2: 100 MVA, $115 \mathrm{kV}, \mathrm{X} 1=\mathrm{X} 2=0.135$ p.u., $\mathrm{X} 0=0.09$ p.u., $\mathrm{PF}=0.98$

- LOAD_1: $115 \mathrm{kV}, 10 \mathrm{MW}, 1 \mathrm{MVAR}$

- LOAD_2: $115 \mathrm{kV}, 10 \mathrm{MW}, 1 \mathrm{MVAR}$

- $\quad$ XFMR_1: $13.8 / 115 \mathrm{kV}, \mathrm{X} 1=\mathrm{X} 0=0.1$

- XFMR_2: $13.8 / 115 \mathrm{kV}, \mathrm{X} 1=\mathrm{X} 0=0.09$

- XFMR_PCC: $500 / 115 \mathrm{Kv}, \mathrm{x} 1=\mathrm{x} 0=0.02$

STEP 2. Add (67) phase relays on both ends of each line and on the network side on each transformer using the relay identification numbers indicated in Table 1. Decide which relays must be regular overcurrent, based on their placement in the system, and set in ASPEN. You can start by using the same settings for each relay inside the loop (there will be different default settings for relays outside the loop):

- Relay Type: JBC51

- CT Ratio: $400 / 5=80$

- TAP: 5 A

- Time Dial: 3

- Instantaneous: $100,000 \mathrm{~A}$

- Directional Time Element: (student discretion) 
- Directional Instantaneous: unchecked

STEP 3. Neglect the fact that the pickup values should be well above load currents. Assume that relay $\mathrm{R} 1$ has a $\mathrm{CTR}=100, \mathrm{TD}=0.5$, and $\mathrm{Ipu}=0.5 \mathrm{~A}$. Assume that relays $\mathrm{R} 5$ and $\mathrm{R} 7$ have a time dial of 1.0 and an $\mathrm{I} \mathrm{pu}=1 \mathrm{~A}$. That relays looking into generator units G1 and G2 are directional with a time dial setting of 0.5 and Ipu $=1 \mathrm{~A}$ with $\mathrm{CTR}=$ 80 .

STEP 4. For the loop, first the coordination pairs must be determined. This is done by performing a close-in, 3-phase faults as relays are analyzed first with the necessary simulations for both clockwise and counter clockwise analysis around the loop, starting at the Equivalent source, to determine the time dial settings of the relays in the loop. Use the example from 12.8 in the Blackburn text, referencing Figure 12.7 as your guide for looped coordination.

Simulate the faults and adjust the time dial and current pick-up until all relays are properly coordinated as explained in lecture theory and Chapter 12 (i.e. section 12.8) of the Blackburn text. If necessary, slightly adjust CTR values.

Make an organized presentation with the relay coordination pairs, as shown in Chapter 12 of Blackburn.

Use a loop coordination interval range of 0.3 to 0.4 seconds for the far-bus faults. Reference suggested operating times for close-in faults outlined in section 12.8. Discuss your criteria in your written report.

**You should use directional units only wherever you think is necessary, and regular OC everywhere else ${ }^{* *}$

\section{Deliverables}

Produce a report with your system screen shot along with the final settings of the relays, and the performance and operational times for each of the faults. Include figures of coordination results. Note that there may be several different solutions to this problem. Discuss how your solution can be improved. 


\title{
B.9 Original Directional Over-current Relays (67) Instructions
}

\author{
Portland State University \\ Electrical \& Computer Engineering \\ ECE 448/548 Power System Protection I
}

-Lab 9. Directional Over-current Relays (67) -

\section{Introduction}

In this laboratory exercise, the student learns about electromechanical and digital directional over-current relays.

\section{Objectives}

At the end of the laboratory exercise the student will be able to do the following:

- Identify the different parts of an electromechanical directional over-current relay

- Determine Time Overcurrent operation of an electromechanical directional element using relay testing equipment and compare results to theoretical calculations for the appropriate relay curve

- Determine the operation zone, based on current angles, of an electromechanical directional element using relay testing equipment

- Implement physical set-up to test SEL-351 using the SEL-RTS (AMS)

- Introduce settings to the relay (Phase and Neutral Ground elements)

- Test the SEL-351 relay with SEL-5401 software and obtain operation times

- Compare results to those found by theoretical calculation

\section{Part I: Testing JBC $51 N$ relay elements}

\section{Materials/Equipment}

- Avo Pulsar relay tester

- Test leads

- Oscilloscope

- Current probe

- JBC $51 \mathrm{~N}$ relay

STEP 1: Download JBC51N instruction manual and identify most relevant parts of the relay. Identify the terminals associated with voltage and current source inputs as well as relay contacts, which will be used in the Timing Test and Directional 
Unit Test.

STEP 2: Download Pulsar manual and reference Table of Contents for relay type. Find relay type. Go to section and read instructions for three of the basic relay testing procedures outlined there:

a. TIMING TEST

b. DIRECTIONAL UNIT

c. INSTANTANEOUS OVERCURRENT PICKUP

STEP 3: Connect oscilloscope to relay testing circuit.

STEP 3: Perform TIMING TEST on the relay from the Pulsar instruction manual Record results for time operation at the specified fault current of $2 \mathrm{x} \mathrm{I}_{\mathrm{PU}}$ tap. Note the difference between the Pulsar ammeter and the oscilloscope readings. Compare to calculated operating time values using equations from lecture material.

$$
\mathrm{t}_{\mathrm{r}}=\mathrm{TD} \cdot\left(\frac{5.95}{1-\mathrm{M}^{2}}\right)
$$

Plot calculated and tested results on the provided relay curve from relay manual (Figure 1). 


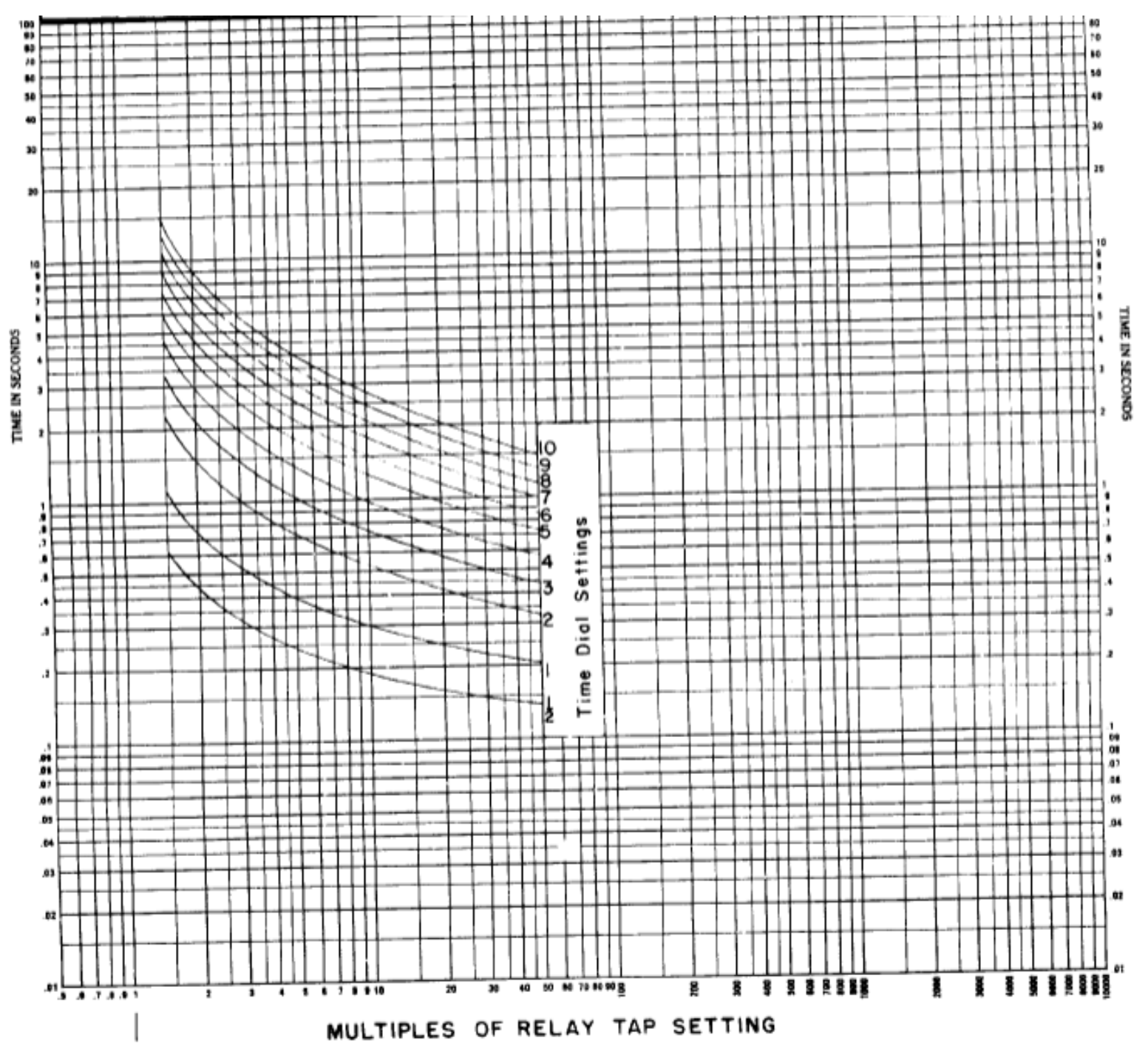

Figure 1: US Inverse Curve U2 from JBC51N instruction manual

STEP 4: Perform DIRECTIONAL UNIT test from Pulsar instruction manual. Record results for IPU value over the range of current angles. Note the difference between the Pulsar ammeter and the oscilloscope readings. Plot both results on the provided relay curve from relay manual. Reference Setting Phase Angle Relationships from the Pulsar instruction manual for this step.

\begin{tabular}{|c|c|c|c|c|c|c|c|c|}
\hline P.F. Angle - Lag & $90-135$ & $135-180$ & $180-225$ & $225-270$ & $270-315$ & $315-360$ & $0-45$ & $45-90$ \\
\hline $\begin{array}{c}\text { Power Flow } \\
\text { KW IN/OUT }\end{array}$ & OUT & $\mathbb{I N}$ & $\mathbb{I N}$ & $\mathbb{I N}$ & $\mathbb{I N}$ & OUT & OUT & OUT \\
\hline \hline
\end{tabular}

Table 1: Current flow direction for lagging current angles 


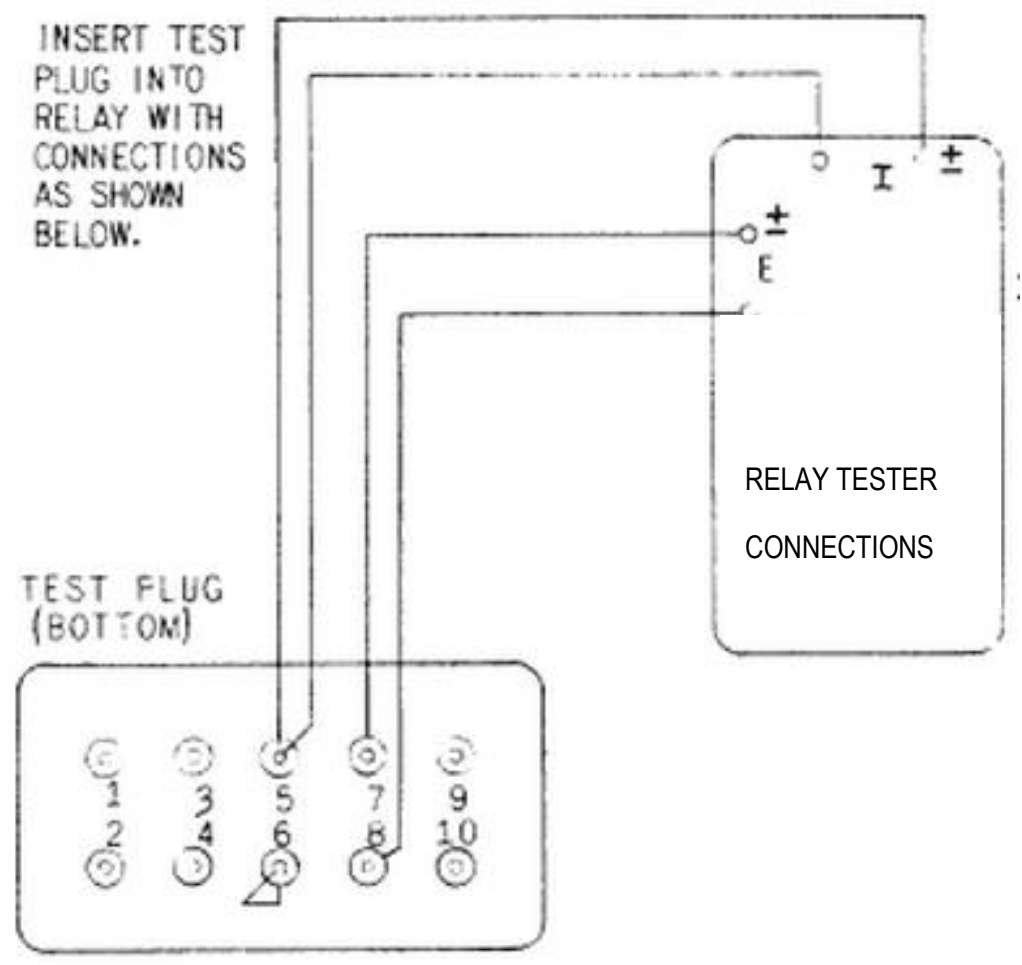

Figure 2: Pin out for directional element test, Figure 24 from JBC $51 \mathrm{~N}$ instruction manual

STEP 5: Perform INSTANTANEOUS OVERCURRENT PICKUP test. Record result and compare to physical relay setting.

\section{Part II: Introductory procedure to test the SEL-351 relay}

\section{Materials/Equipment}

- SEL-351 Protection System relay

- SEL-AMS

- SEL-5401

- AcSELerator QuickStart software

- C724 ribbon cable

- C234A serial port cable

- C662 USB to serial port cable

STEP 1. Download a copy of the SEL-351 instruction manual. Read the introduction and the part that corresponds to protection elements.

STEP 2. Open AcSELerator Quickset and set the SEL-351 according to the table shown in the next pages pages 2 and 3 of this document).

STEP 3. Connect the AMS to the SEL-351, using the procedure learned in previous 
laboratory exercises using the appropriate ribbon cable.

STEP 4. Connect the SEL-351 to the computer using the proper cable (C662), and establish communication with the relay using the proper communication settings in the AcSELerator software. Use the knowledge you acquired in the previous digital relay laboratory exercise and the settings listed at the end of this instruction sheet. If the relay password is needed, reference relay instruction manual for Default Passwords. If the C662 cable is unavailable, input setting to relay front panel manually.

STEP 5. Connect the AMS testing system to the computer as in previous laboratory exercises with the C234-A serial cable. Open the SEL-5401 program, go to UUT Database and update your SEL-351 relay to be a 5 Amp device. Verify Scale Factors have been adjusted, save and close the UUT Database window and then select the SEL-351 relay model from the menu for the new settings. Before setting the State Values (IA, IB, IC, IN), perform the METER test with the Front Panel command on the tool bar, as done in the previous digital relay laboratory exercise. Verify that the values shown on the relay front panel meter (in primary units) are consistent with the CTR programmed into the SEL-351. If needed, use the SEL-AMS instruction manual as a guide.

STEP 6. If Front Panel METER test results are inaccurate, adjust the Scale Factor of the SEL-351 proportionally until values read as expected. Each time the Scale Factor is adjusted, a new case with a new SEL-351 relay must be opened to include this adjusted Scale Factor.

STEP 7. Apply the fault values in the SEL-5401 software as given in Tables $1-3$ below. Case 1 is a balanced $3-\varphi$ fault. Cases 2 and 3 correspond with the faults used in Problem 1, Homework 5.

\begin{tabular}{|c|c|c|}
\hline Quantity & $\begin{array}{c}\text { Magnitude } \\
\text { (Primary kV and amps) }\end{array}$ & Angle (degrees) \\
\hline $\mathrm{V}_{\mathrm{A}}$ & 13.2 & 0 \\
\hline $\mathrm{V}_{\mathrm{B}}$ & 13.2 & -120 \\
\hline $\mathrm{V}_{\mathrm{C}}$ & 13.2 & 120 \\
\hline $\mathrm{I}_{\mathrm{A}}$ & 3323 & -90 \\
\hline $\mathrm{I}_{\mathrm{B}}$ & 3323 & 150 \\
\hline $\mathrm{I}_{\mathrm{C}}$ & 3323 & 30 \\
\hline
\end{tabular}

Table 2: Case 1 fault parameters 


\begin{tabular}{|c|c|c|}
\hline Quantity & $\begin{array}{c}\text { Magnitude } \\
\text { (Primary kV and amps) }\end{array}$ & Angle (degrees) \\
\hline $\mathrm{V}_{\mathrm{A}}$ & 16 & -6 \\
\hline $\mathrm{V}_{\mathrm{B}}$ & 76 & -120 \\
\hline $\mathrm{V}_{\mathrm{C}}$ & 76 & 120 \\
\hline $\mathrm{I}_{\mathrm{A}}$ & 4389 & -80 \\
\hline $\mathrm{I}_{\mathrm{B}}$ & 40 & -84 \\
\hline $\mathrm{I}_{\mathrm{C}}$ & 40 & -84 \\
\hline
\end{tabular}

Table 3: Case 2 fault parameters

\begin{tabular}{|c|c|c|}
\hline Quantity & $\begin{array}{c}\text { Magnitude } \\
\text { (Primary kV and amps) }\end{array}$ & Angle (degrees) \\
\hline $\mathrm{V}_{\mathrm{A}}$ & 21.4 & -8 \\
\hline $\mathrm{V}_{\mathrm{B}}$ & 76 & -120 \\
\hline $\mathrm{V}_{\mathrm{C}}$ & 76 & 120 \\
\hline $\mathrm{I}_{\mathrm{A}}$ & 1238 & 98 \\
\hline $\mathrm{I}_{\mathrm{B}}$ & 24 & 109 \\
\hline $\mathrm{I}_{\mathrm{C}}$ & 31 & 111 \\
\hline
\end{tabular}

Table 4: Case 3 fault parameters

\section{Deliverables}

Produce a short report with summary of results, including operation time calculations and the hand plotted results on the Time-Current curves, for parts 1 and 2 comparing experimental values to theoretical values. Include the figures of Relay Configuration window, the UUT report results, and the AcSELerator QuickSet settings and a discussion of the tested results compared to the expected results from Homework 5 for Cases A and B. Report is due EOB on Friday, March 22nd.

$* * * * * * * * * * * * * * * * * * * * * * * * * * * * * * * * * * * * * * * * * * * * * * * * * * * * * * * * * * * * * * * * * * * * * * *$

$* * * * * * * * * * * * * * * * * * * * * * * * * * * * * * * * * * * * * * * * * * * * * * * * * * * * * * * * * * * * * * * * * * * * * * *$ ***************************************************************************

In AcSELerator QuickStart, begin by creating New Settings for SEL-351:

SEL-351-6 --> 100 --> $>$ Okay $>$

In the next window, correct settings to correspond $w / P / N$ of relay: $035163 A 3 C 542 X 1$.

Use the following settings to program the SEL-351 for testing:

Set 1

General Settings
Setting

Symbol

Description

CTR Phase Current Xfmr Ratio

CTRN Neutral Phase Current Xfmr Ratio
Units Value

N/A $\quad 50$

N/A $\quad 50$




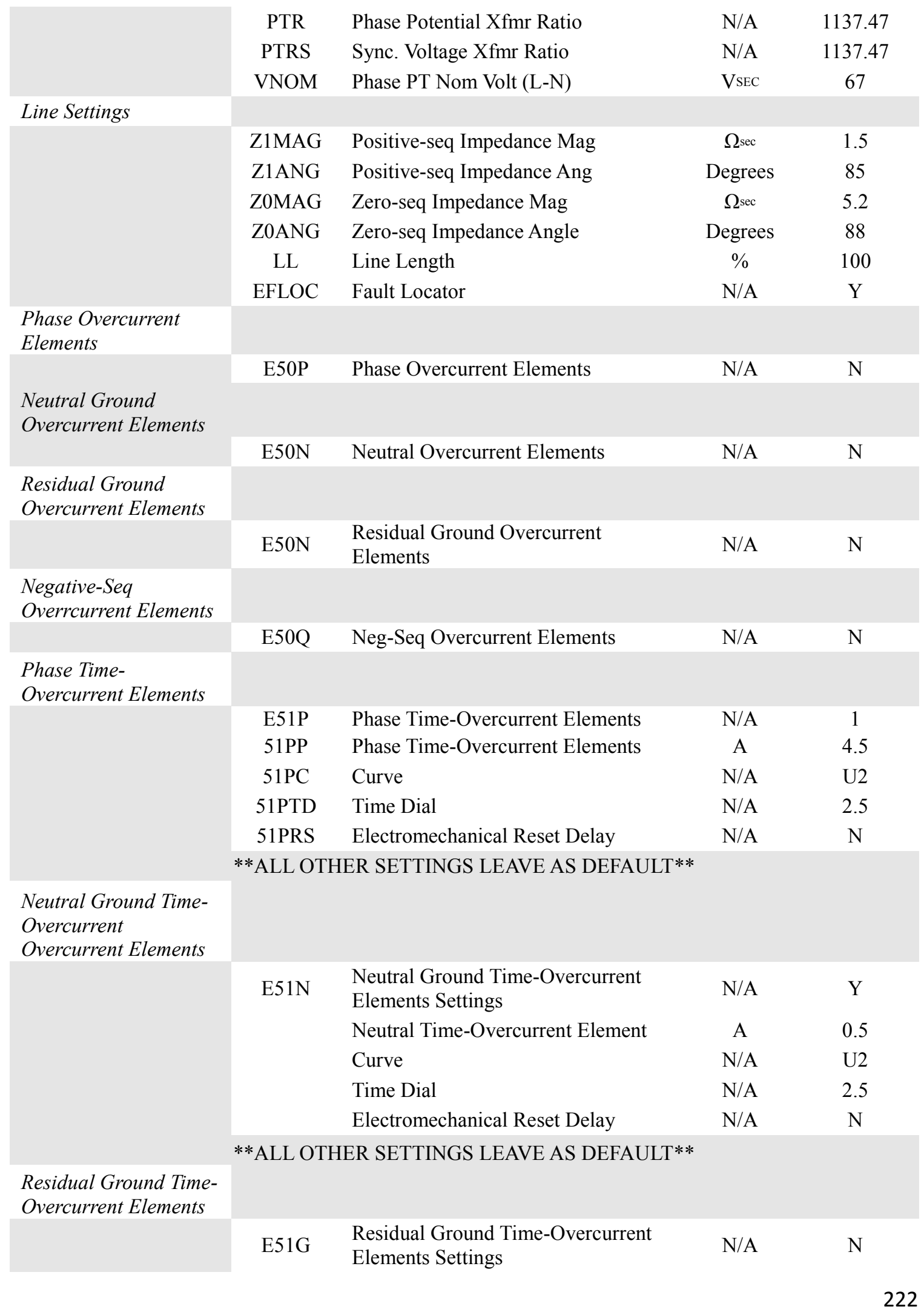




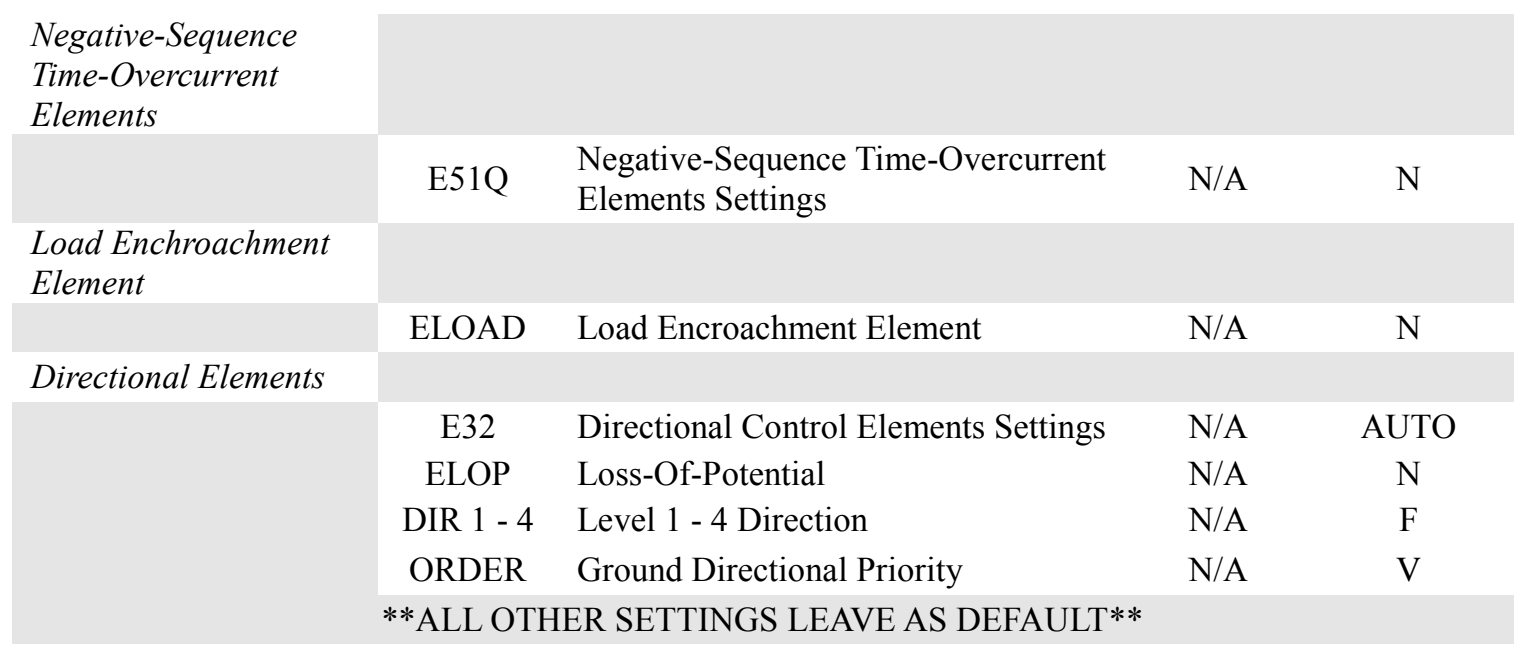

\section{Leave all other Set 1 settings as default}

For the Logic 1 variable settings,

$>51 \mathrm{PTC}=1 \ldots$ permanently activate directional control on $51 \mathrm{P}$ elements

$>51 \mathrm{GTC}=1 \ldots$ permanently activate directional control on $51 \mathrm{G}$ elements

$>\mathrm{TR}=51 \mathrm{PT}+51 \mathrm{NT} \ldots \mathrm{TRIP}$ signal just when 51 or $51 \mathrm{~N}$ operate

$>\mathrm{SV} 6=32 \mathrm{PF}+32 \mathrm{QF}$... an internal logic variable which becomes 1 when the positive sequence directional element or the negative sequence directional element indicate forward direction

$>$ Outputs....turn on applicable outputs which will be tested as phases A, B, C, and $\mathrm{N}$.

For the Global 1 settings,

$>\mathrm{LER}=30$

$>\mathrm{PTCONN}=\mathrm{WYE}$ 
Appendix C: Phase I - Laboratory Design Drawings 

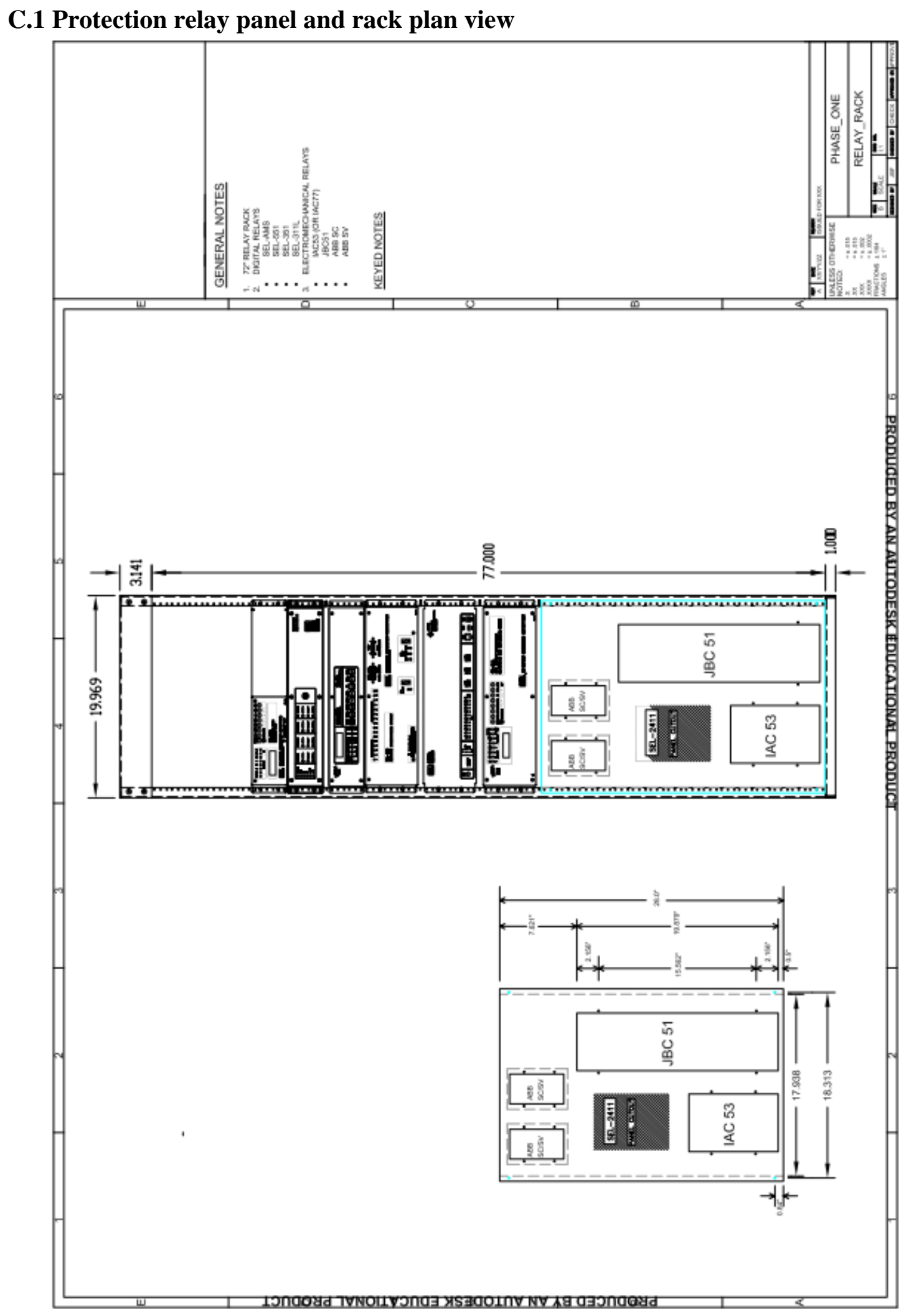

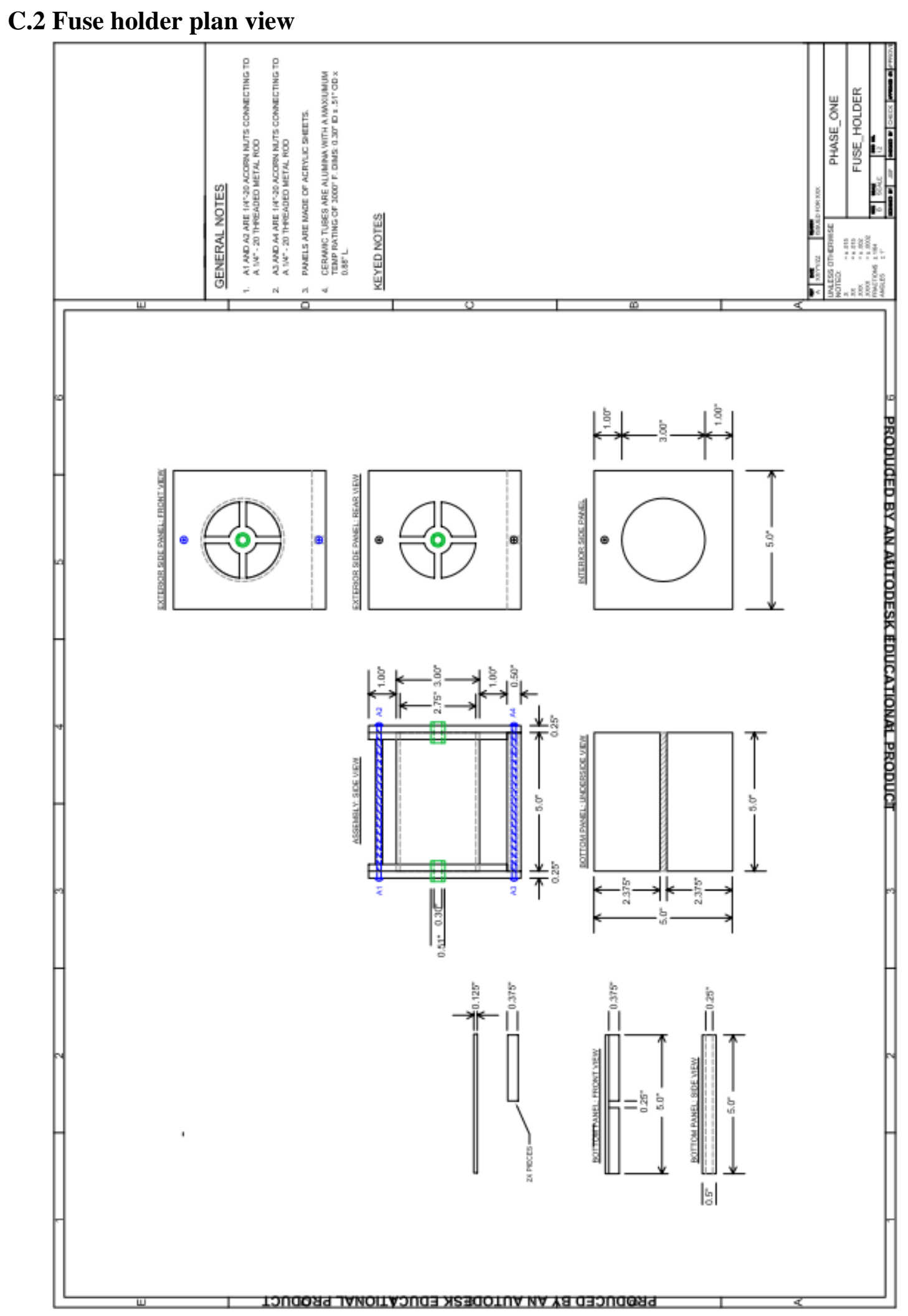
Appendix D: Phase III - Laboratory-Scale Power System Design Drawings 
D.1 Phase III model-scale power system one-line drawing

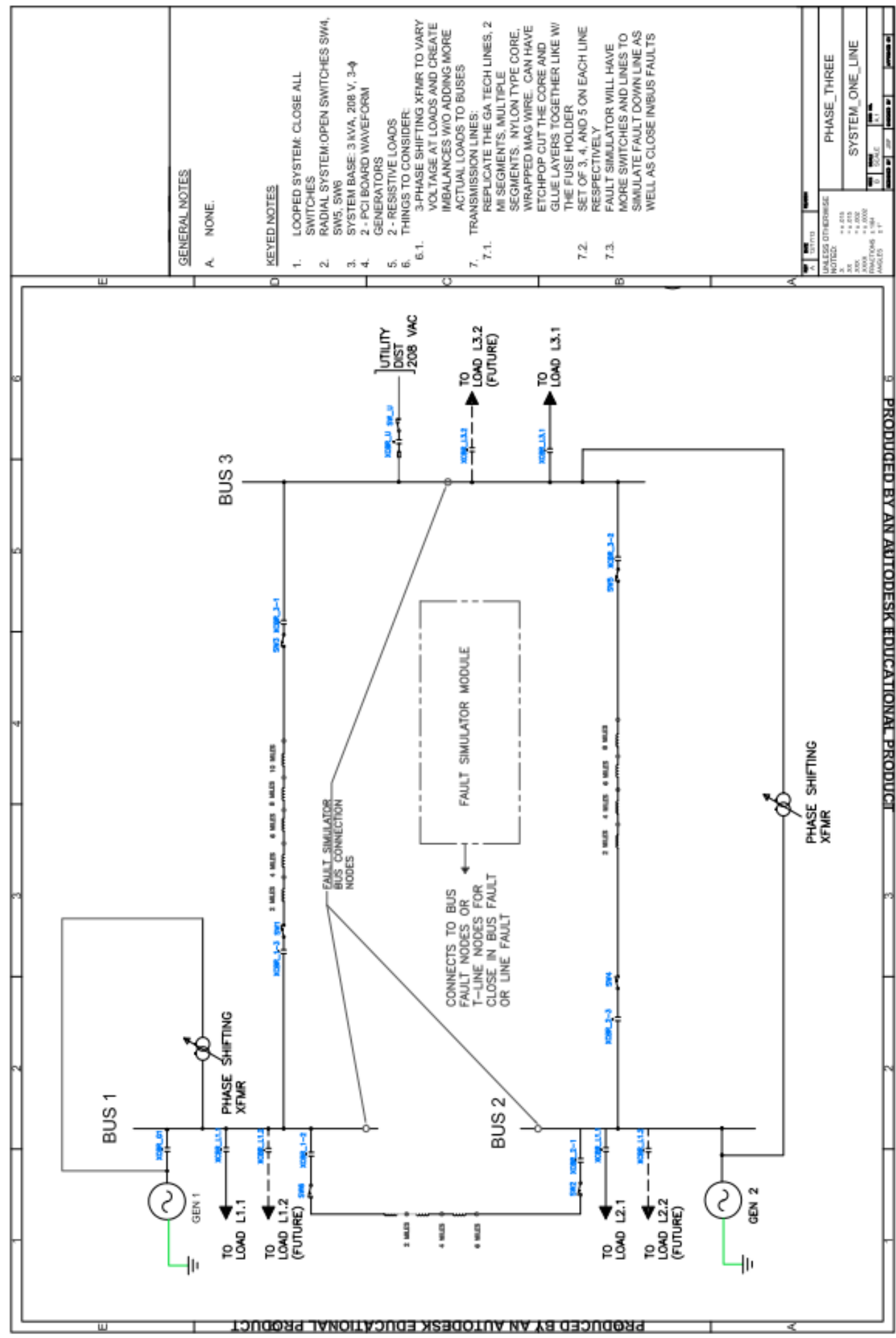




\section{D.2 Phase III model-scale power system three-phase line drawing}

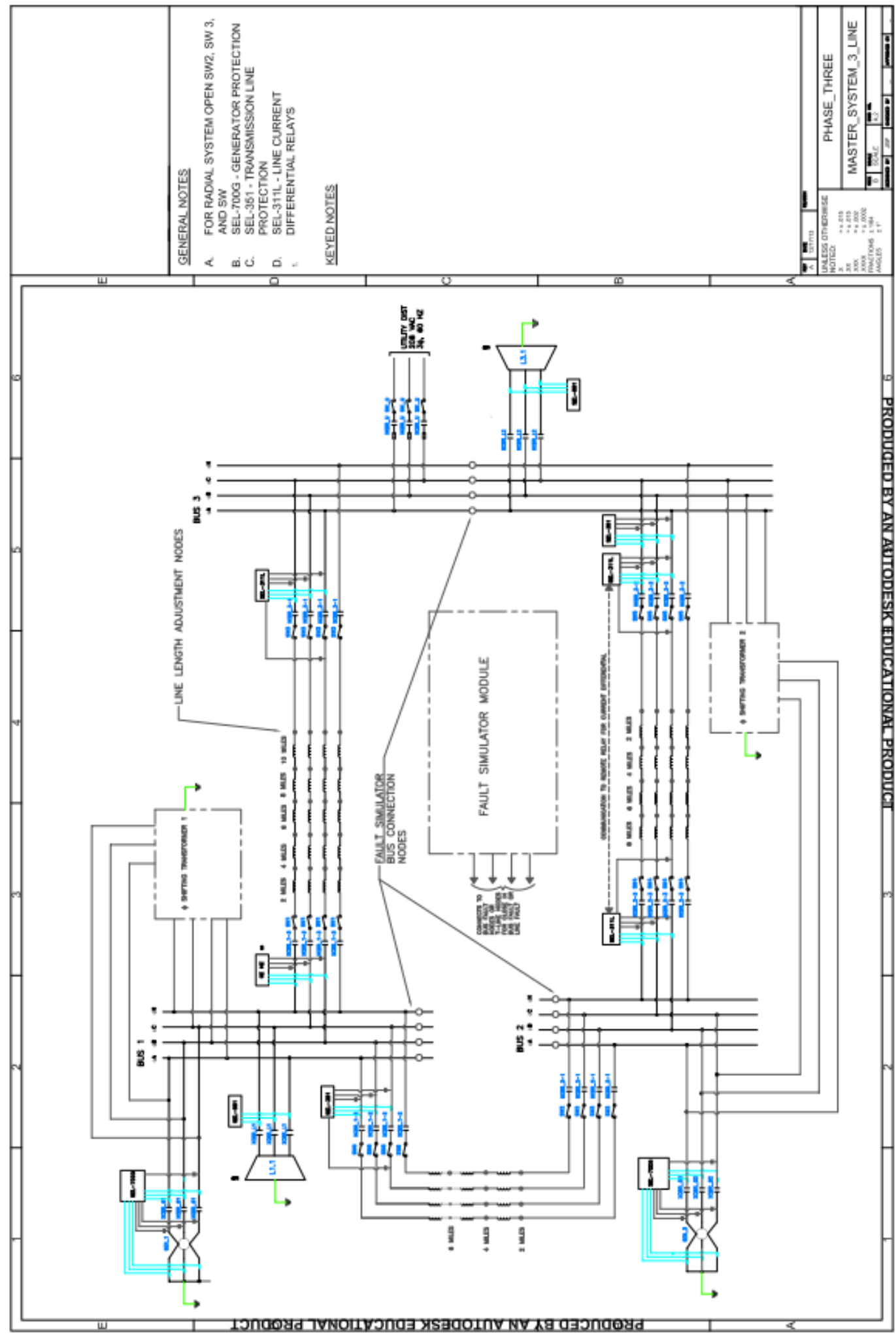




\section{D.3 Phase III model-scale power system Bus 1 three-phase wiring diagram}

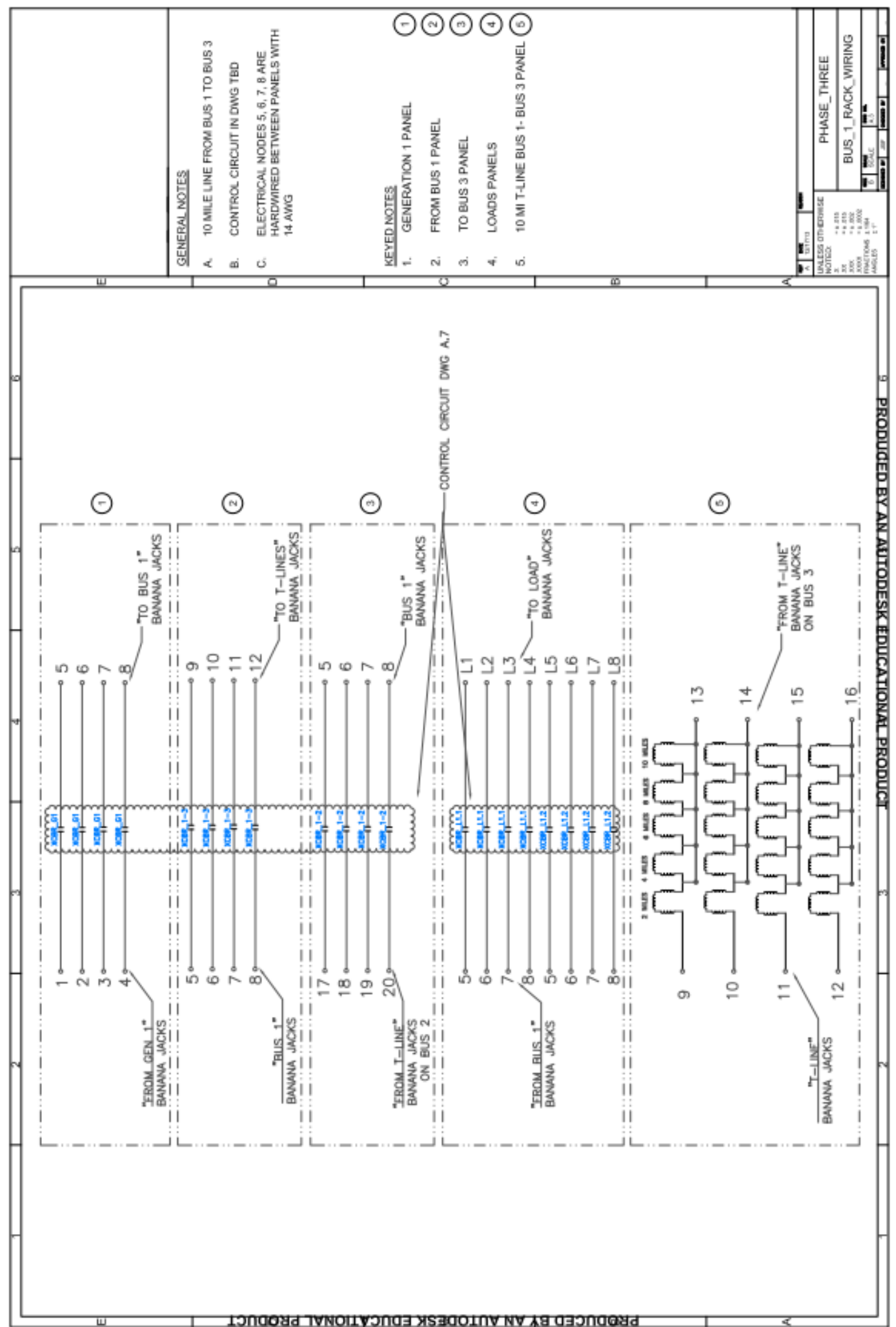




\section{D.4 Phase III model-scale power system Bus 2 three-phase wiring diagram}

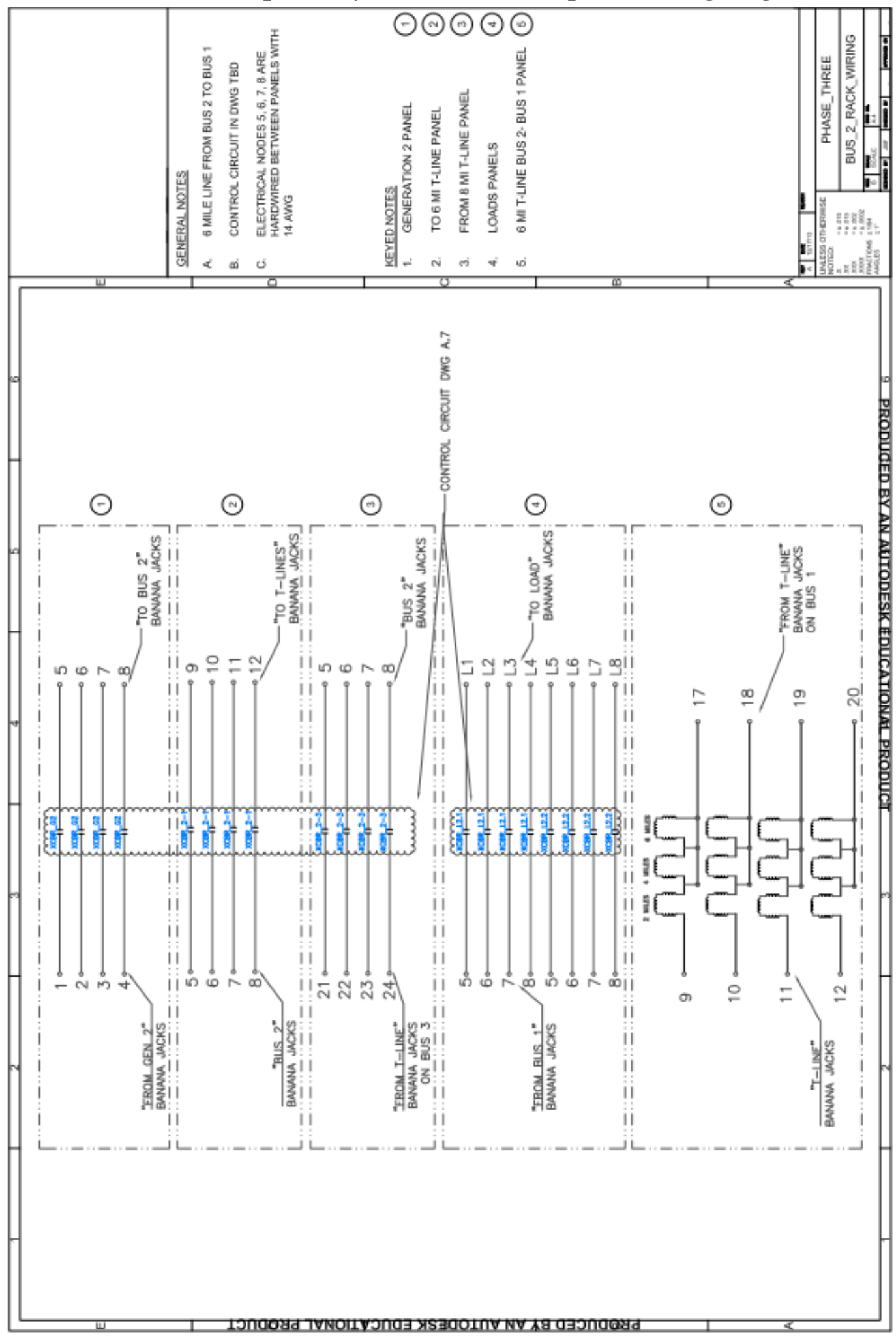




\section{D.5 Phase III model-scale power system Bus 3 three-phase wiring diagram}

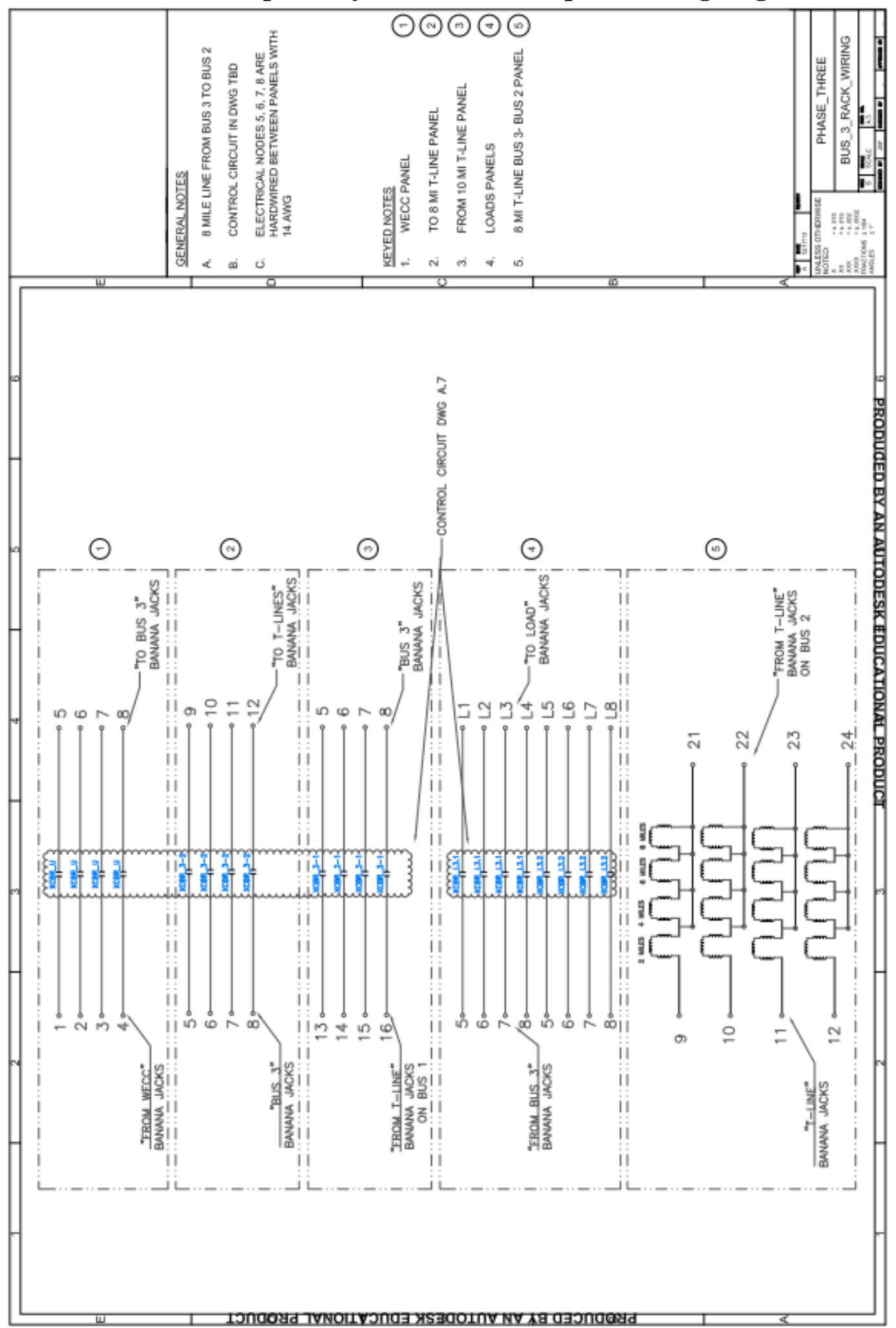




\section{D.6 Phase III model-scale power system generation transformer bank}

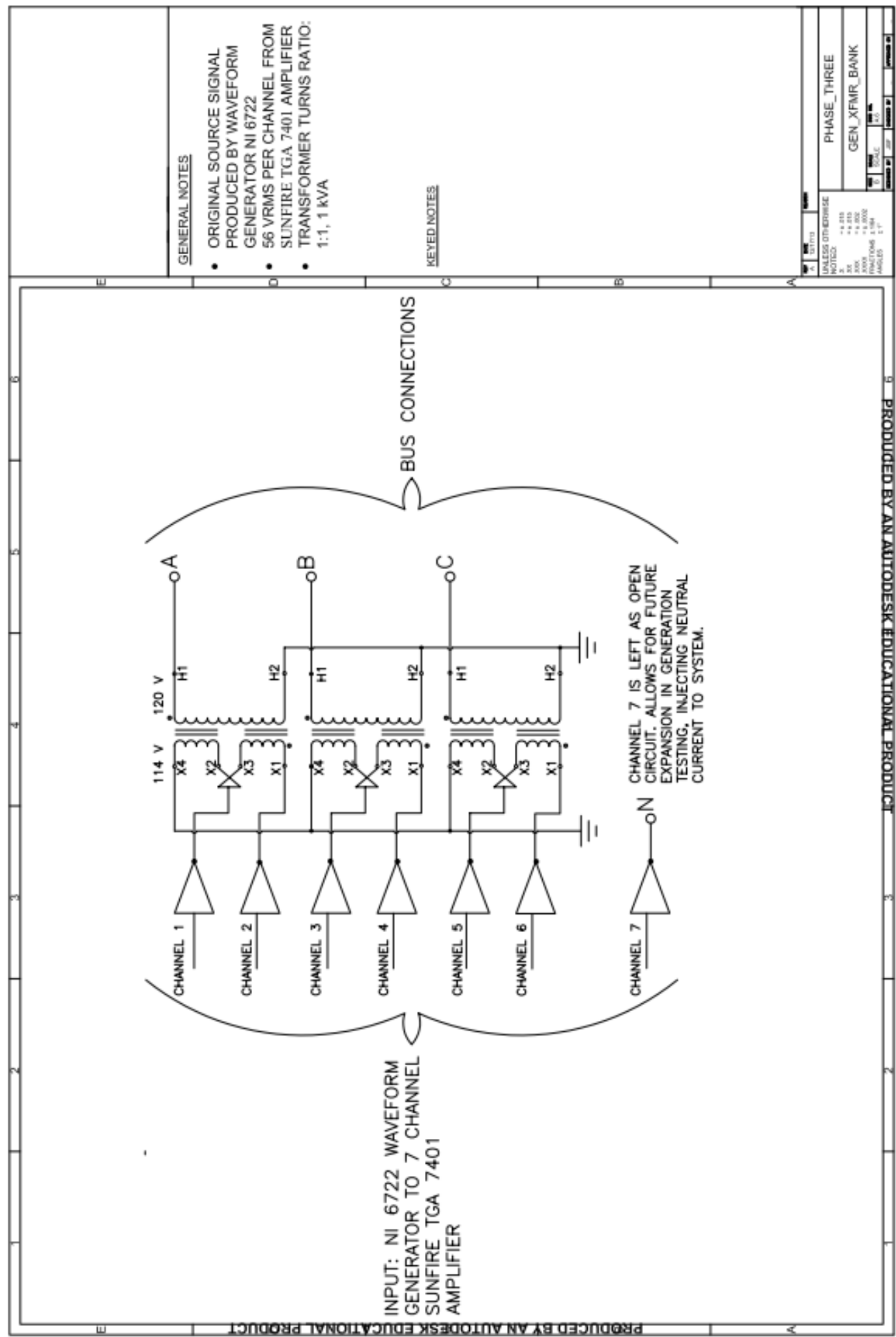




\section{D.7 Phase III model-scale power system circuit breaker control circuit diagram}

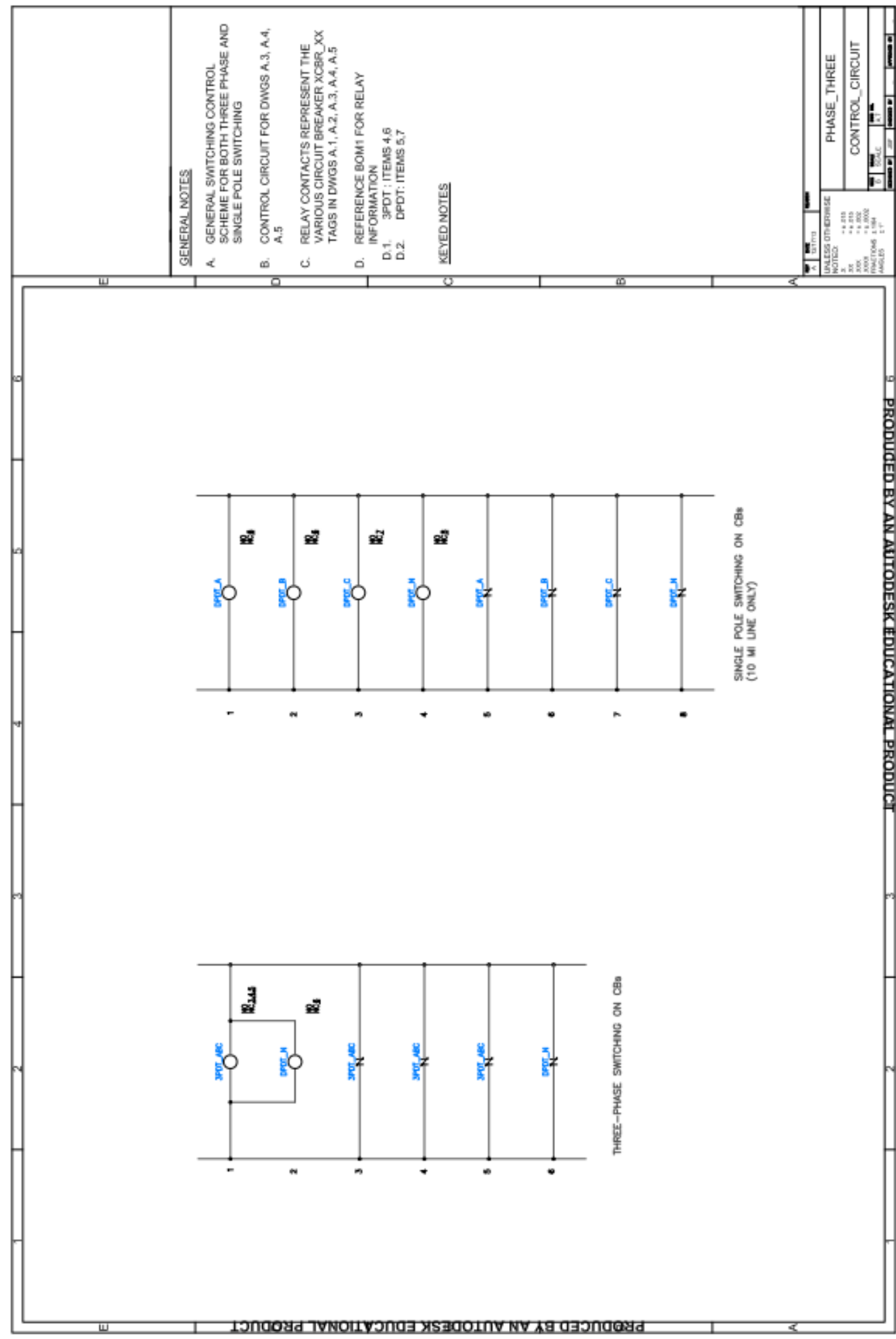


D.8 Phase III model-scale power system load shifting transformer bank

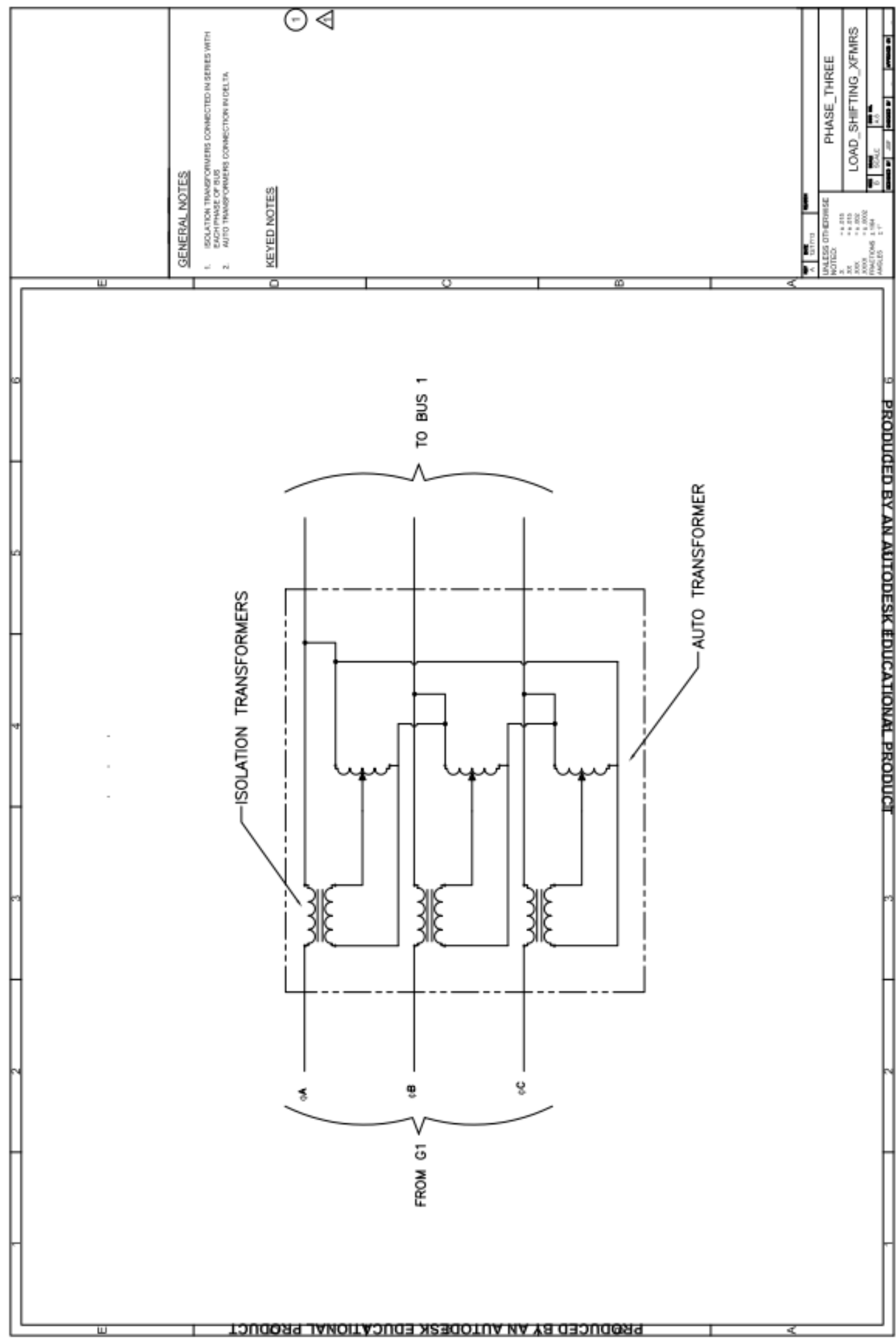




\section{D.9 Phase III model-scale power system generic fault simulator wiring diagram}
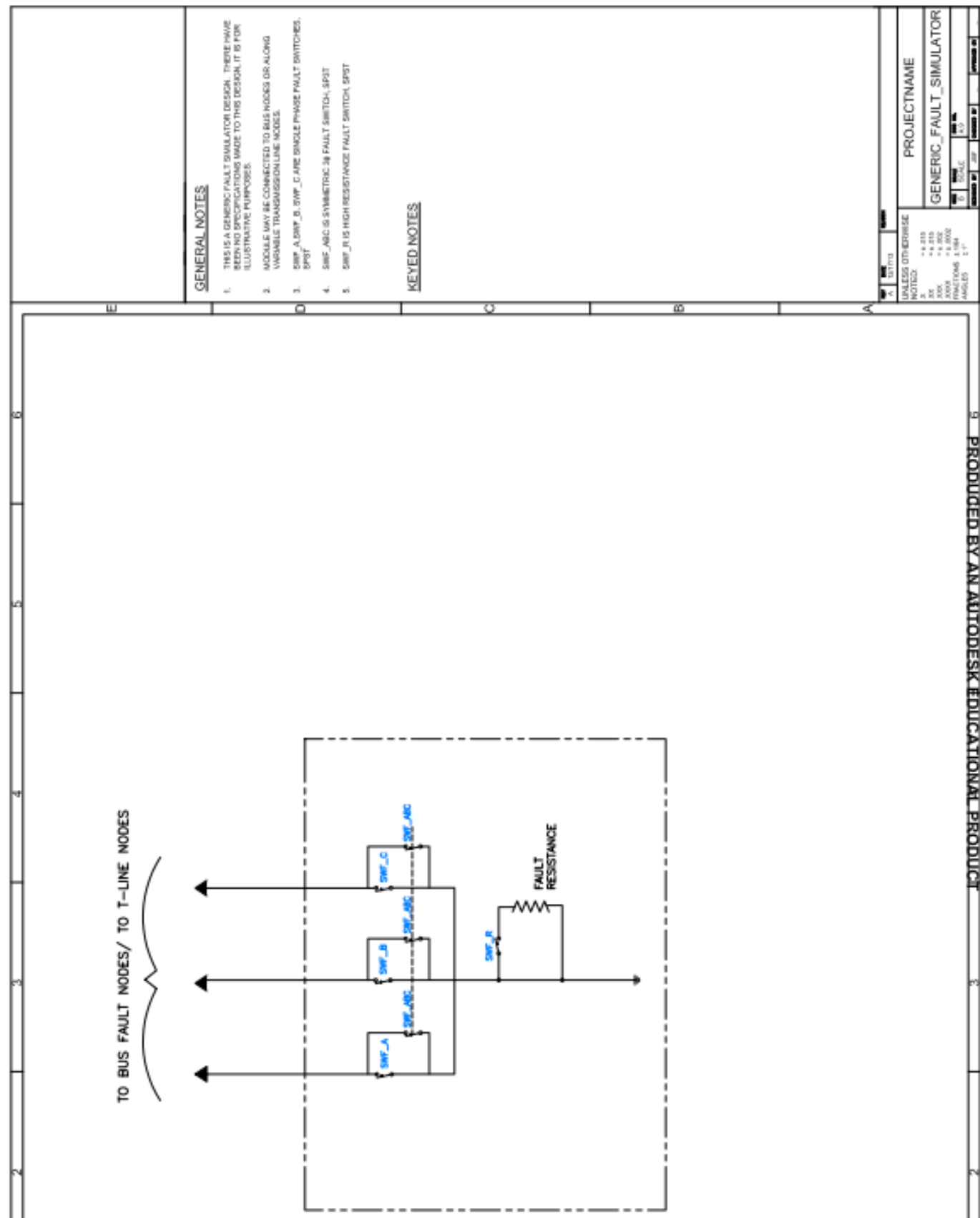


\section{D.10 Phase III model-scale power system Bus 1 plan drawing}

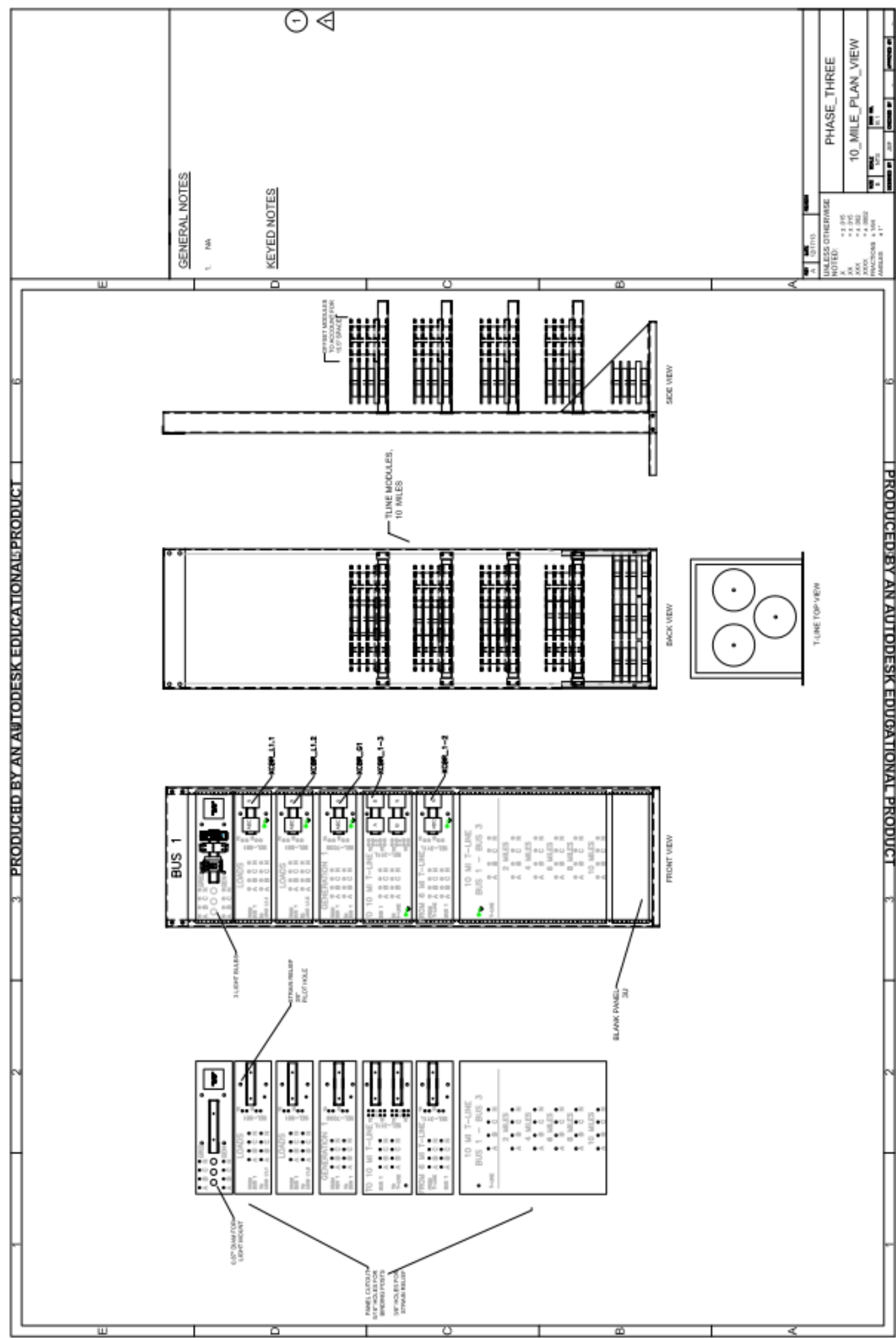


D.11 Phase III model-scale power system Bus 2 plan drawing

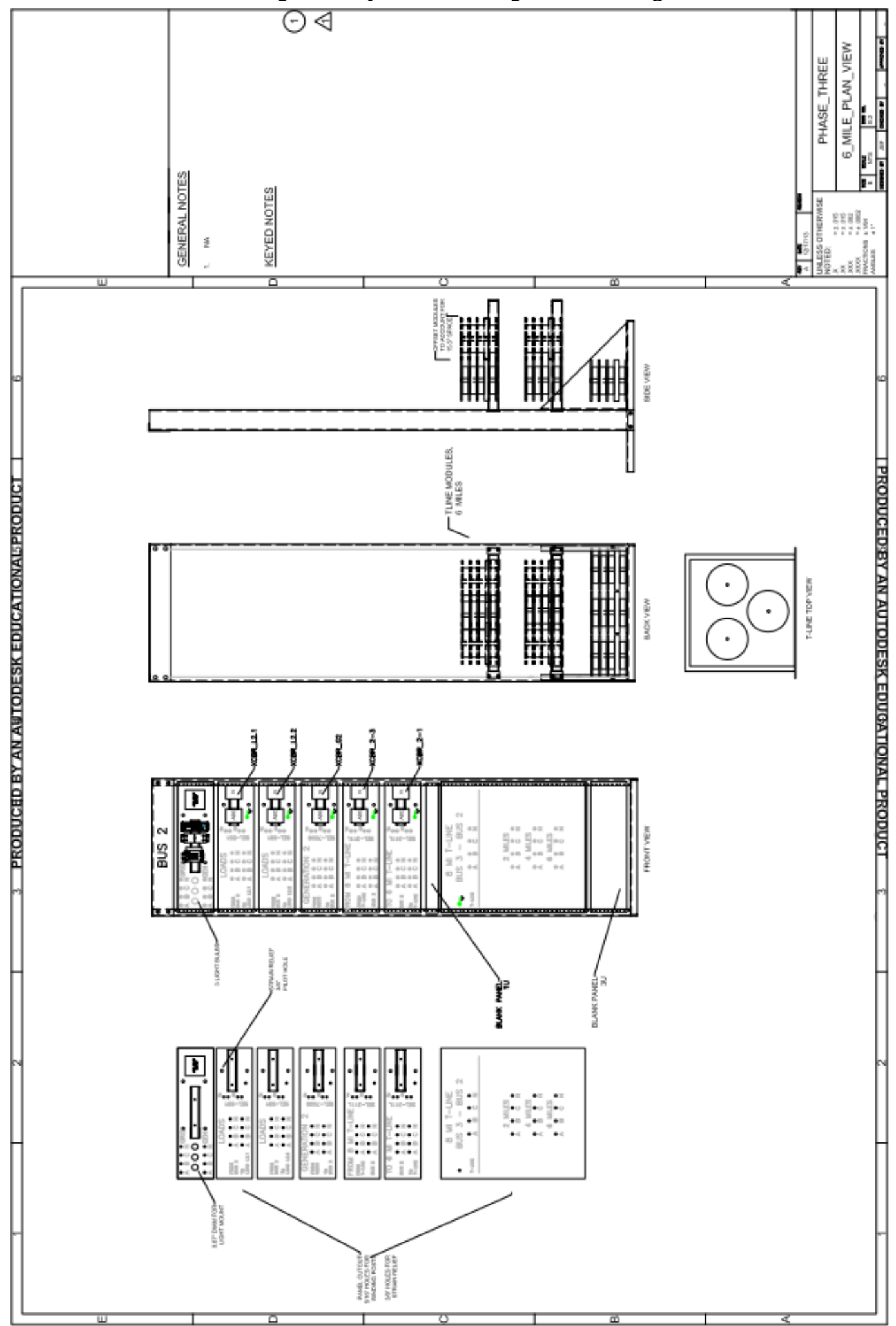




\section{D.12 Phase III model-scale power system Bus 3 plan drawing}

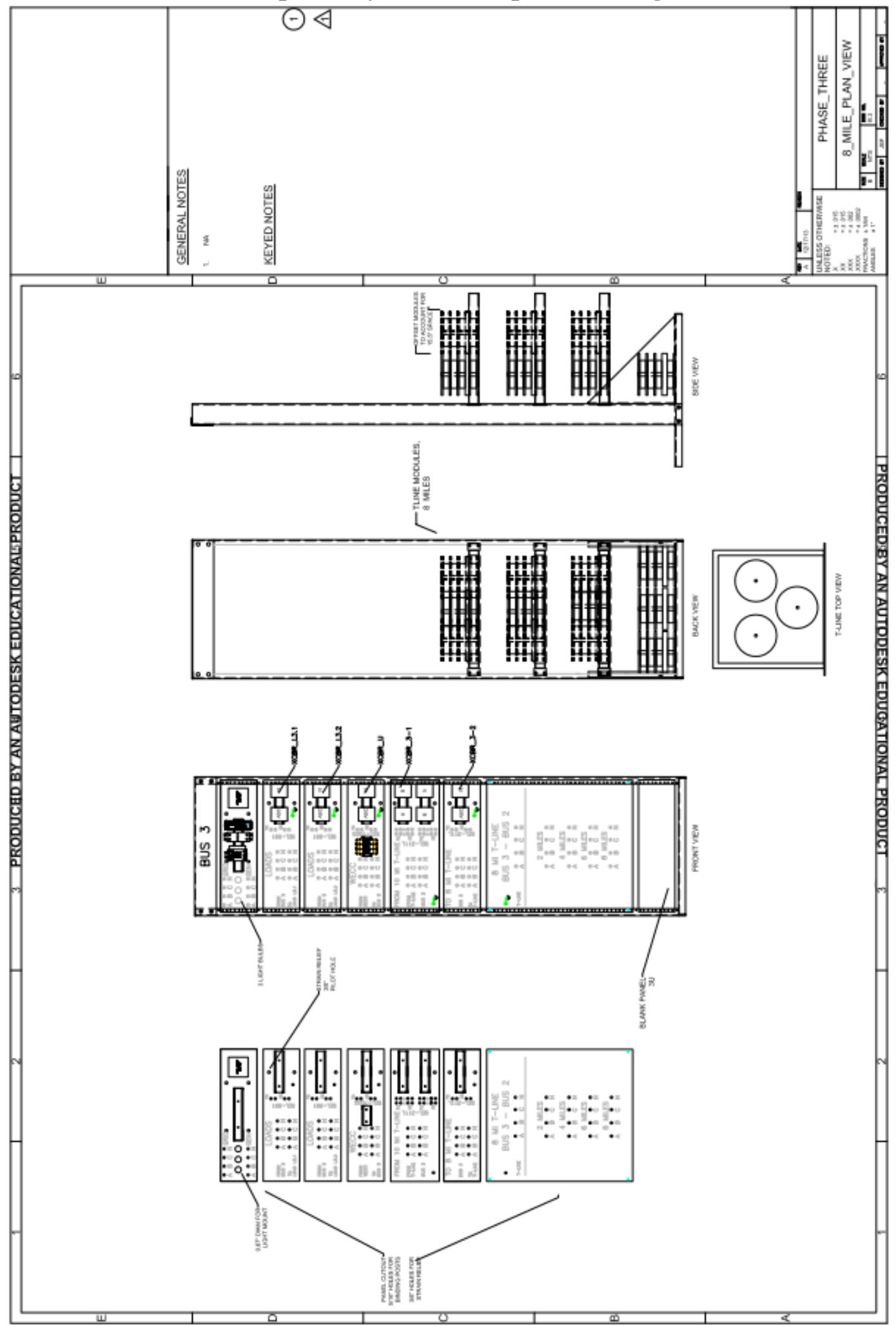




\section{D.13 Phase III model-scale power system load shifting transformer plan drawing}

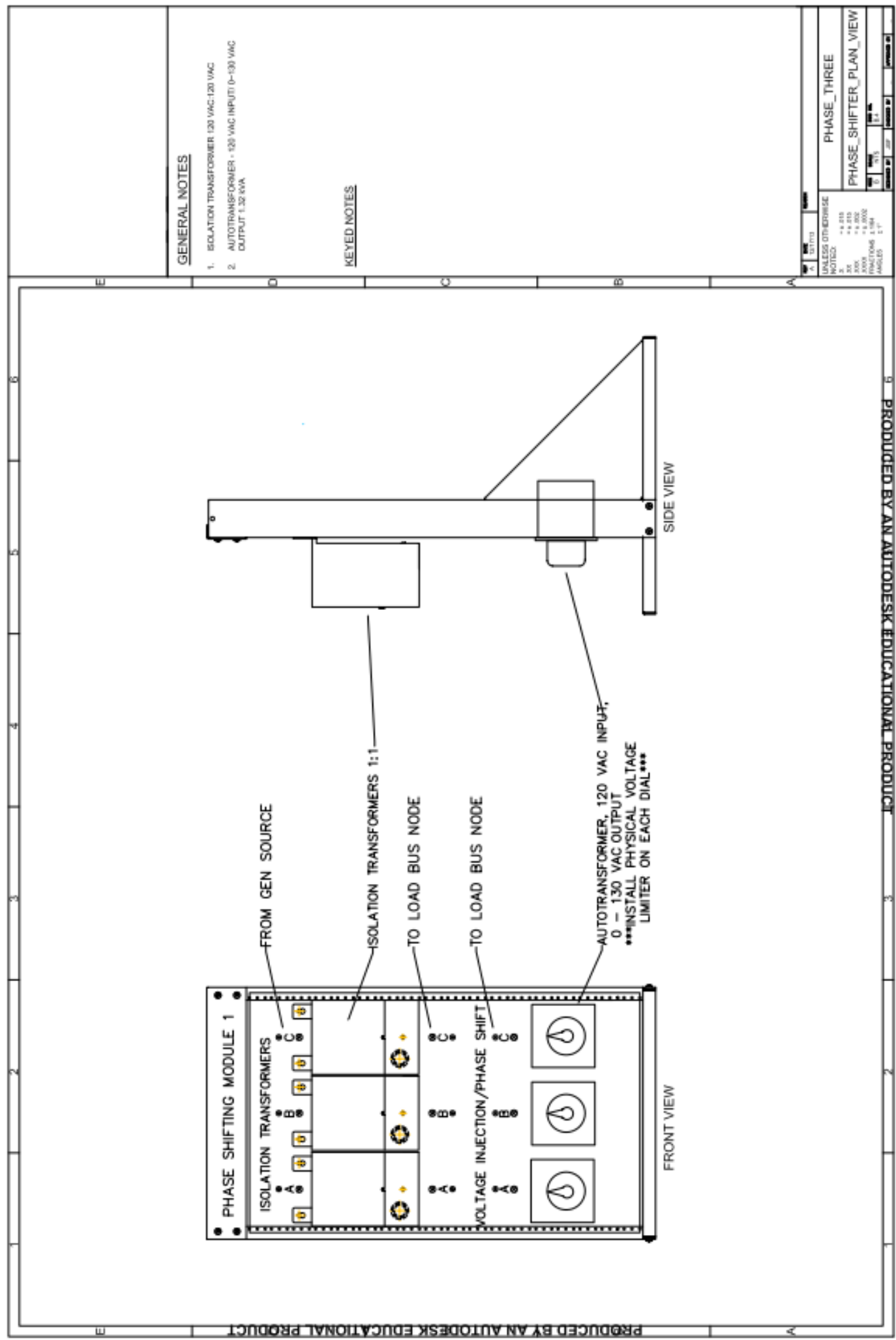


D.14 Phase III model-scale power system transmission line module plan drawing

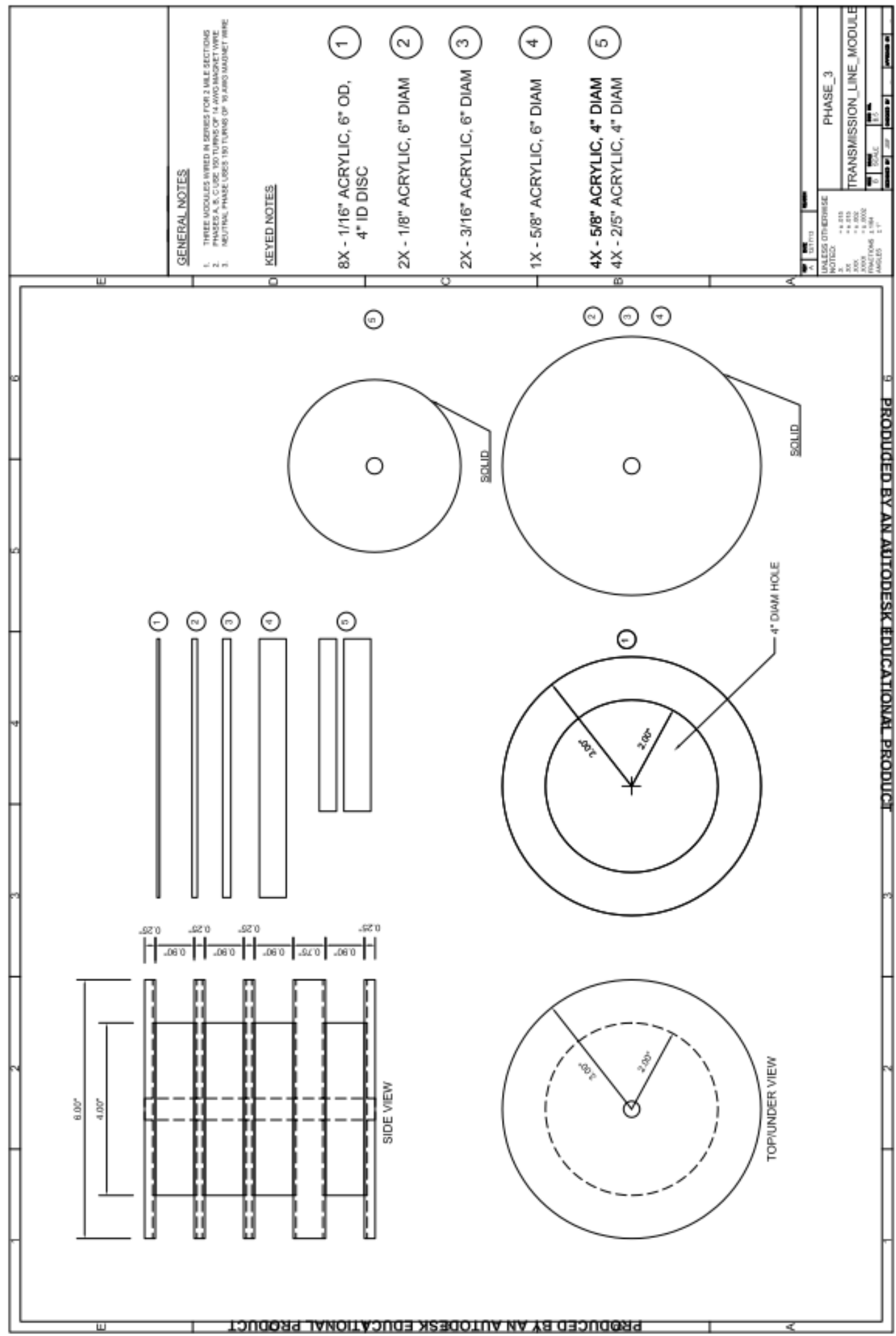


D.15 Phase III model-scale power system 10 mile transmission line panel drawing
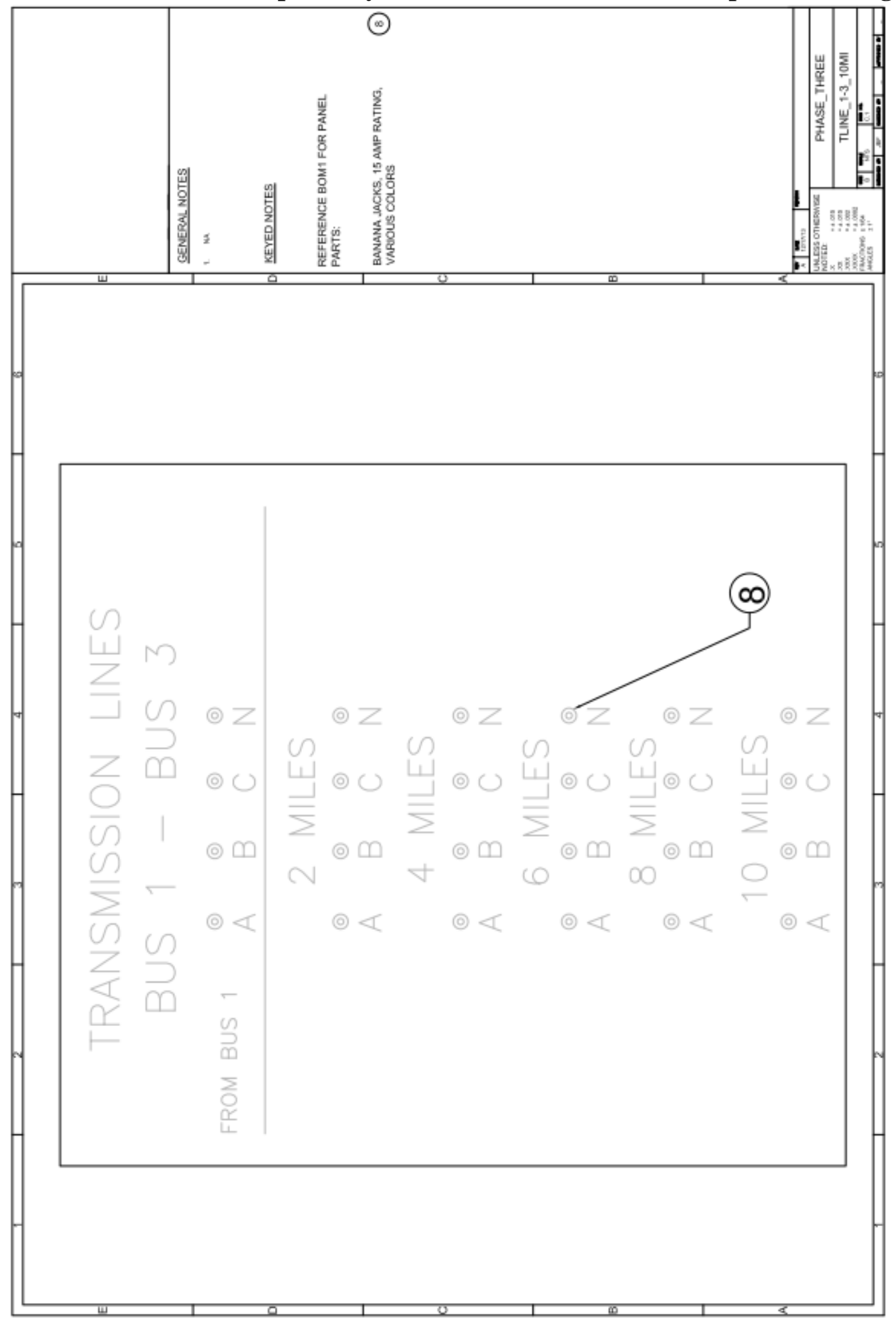
D.16 Phase III model-scale power system 8 mile transmission line panel drawing

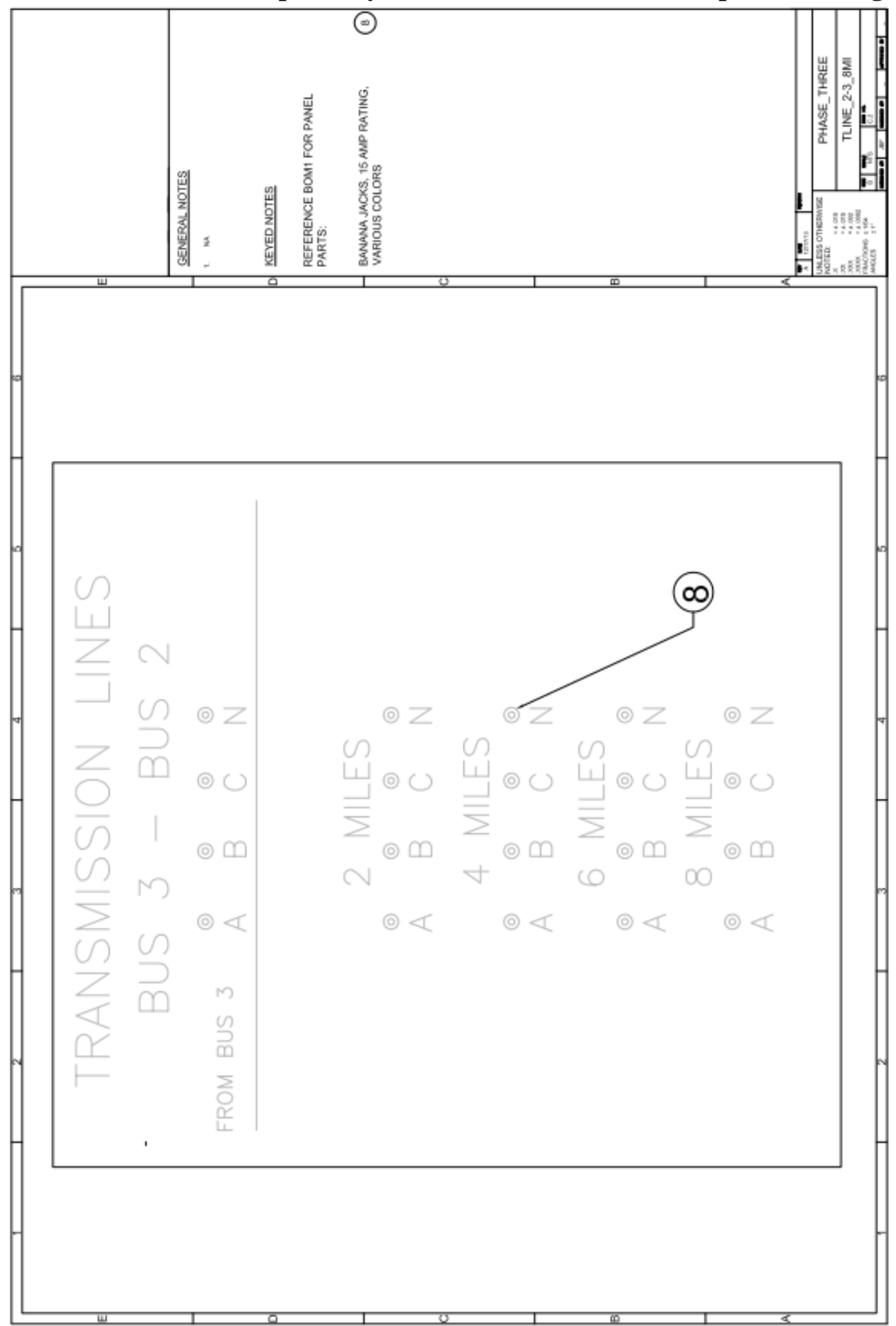


D.17 Phase III model-scale power system 6 mile transmission line panel drawing

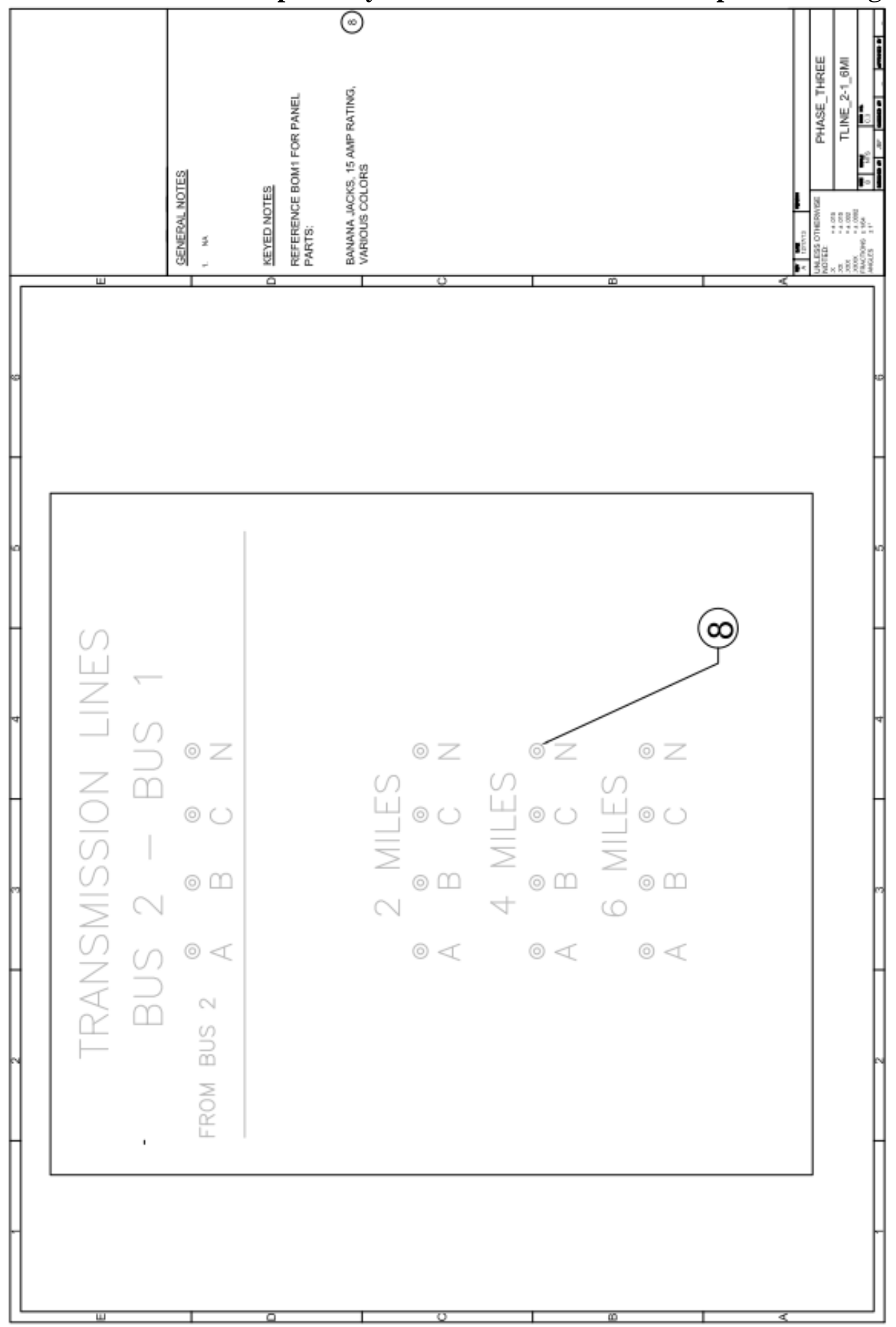




\section{D.18 Phase III model-scale power system Generation 1 panel drawing}

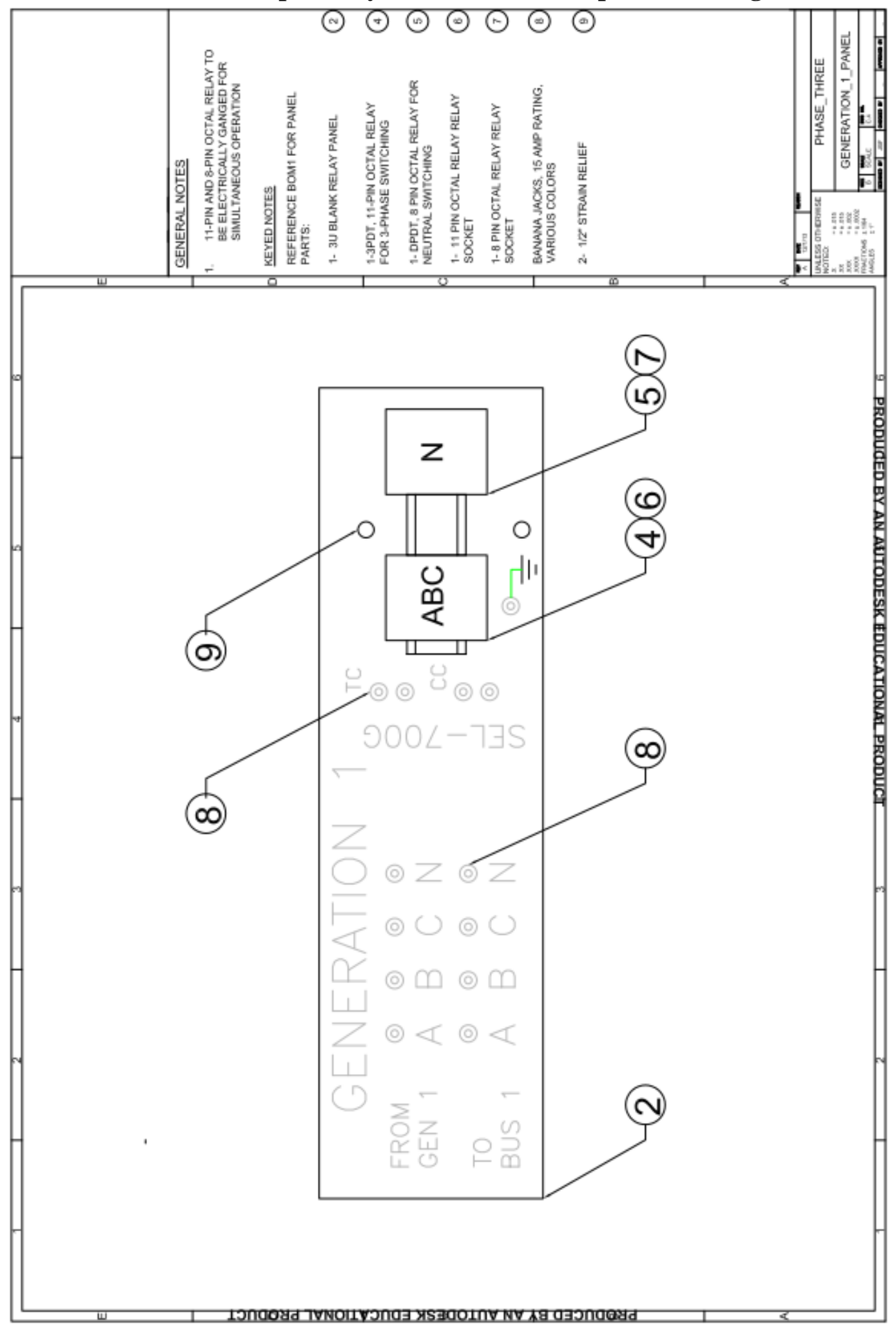




\section{D.19 Phase III model-scale power system Generation 2 panel drawing}

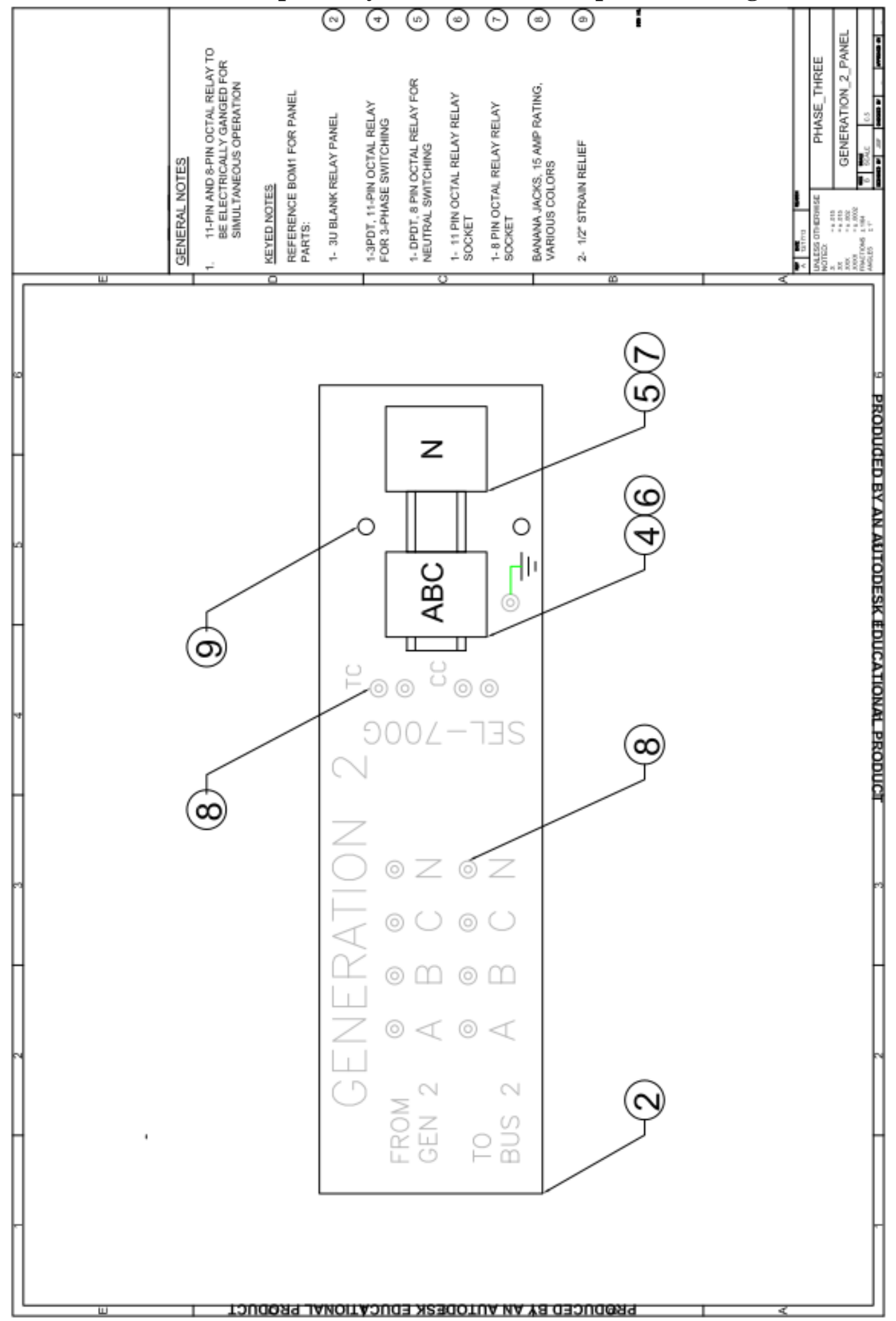



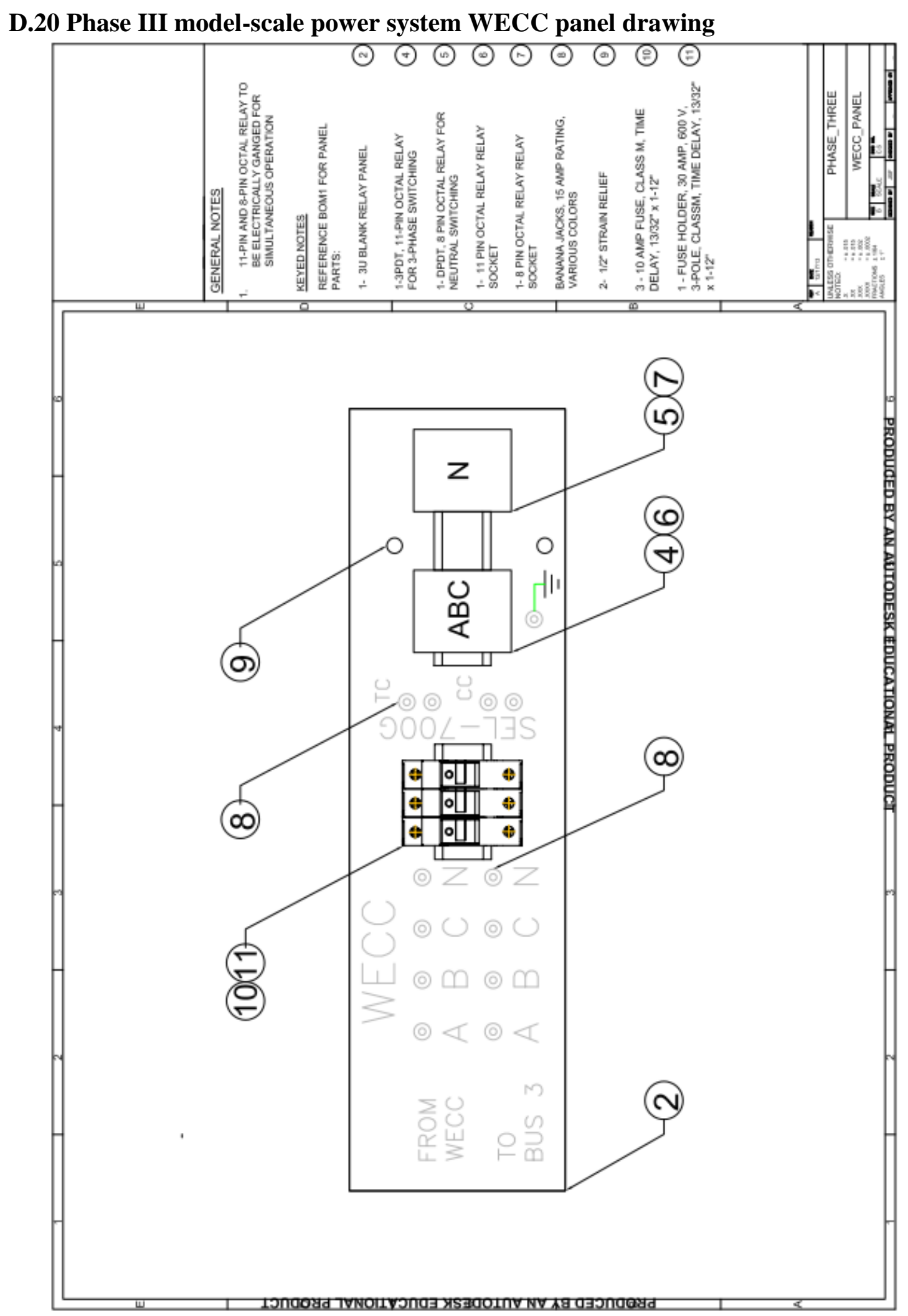
D.21 Phase III model-scale power system Loads panel drawing

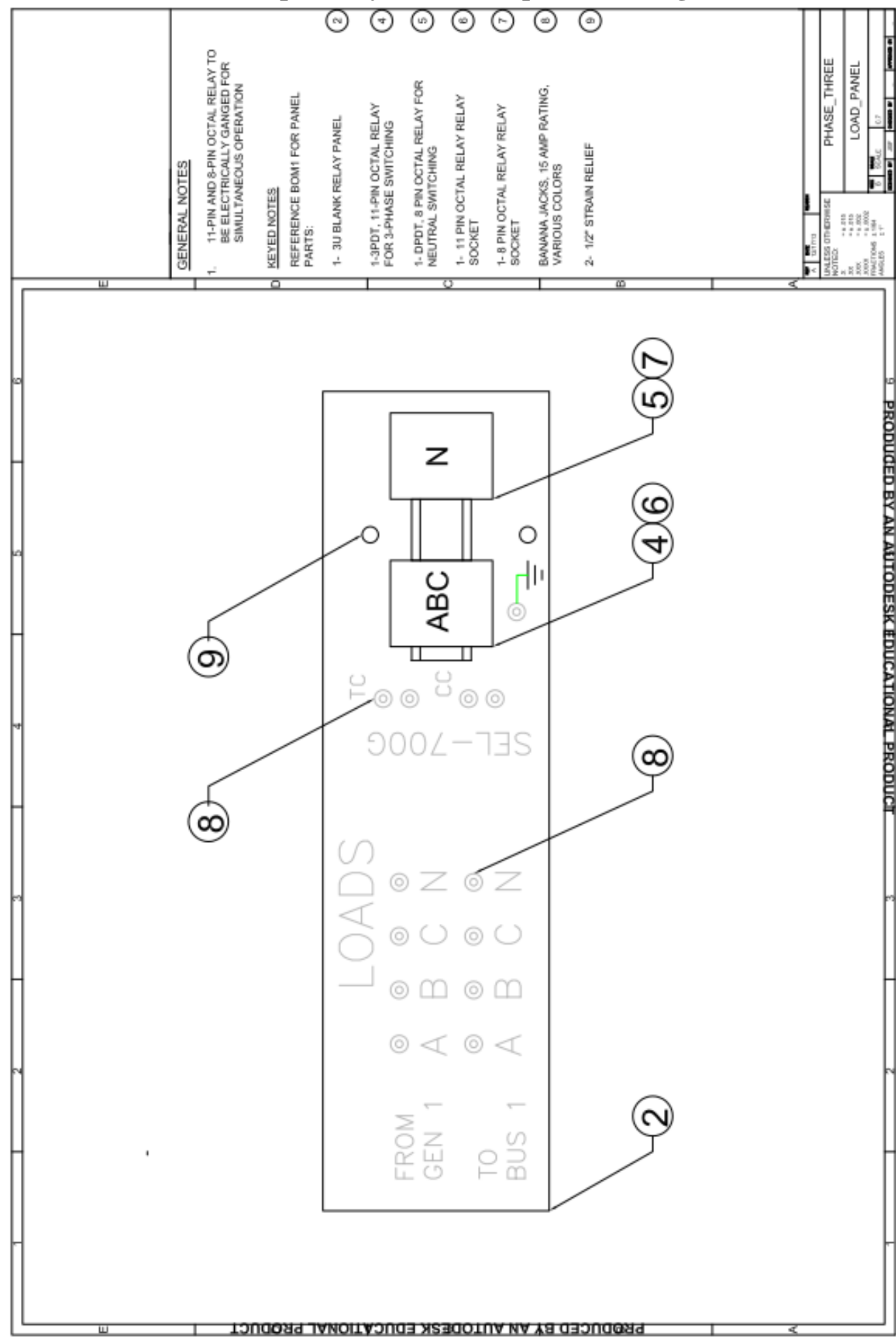




\section{D.22 Phase III model-scale power system From 10 Mile Line panel drawing}

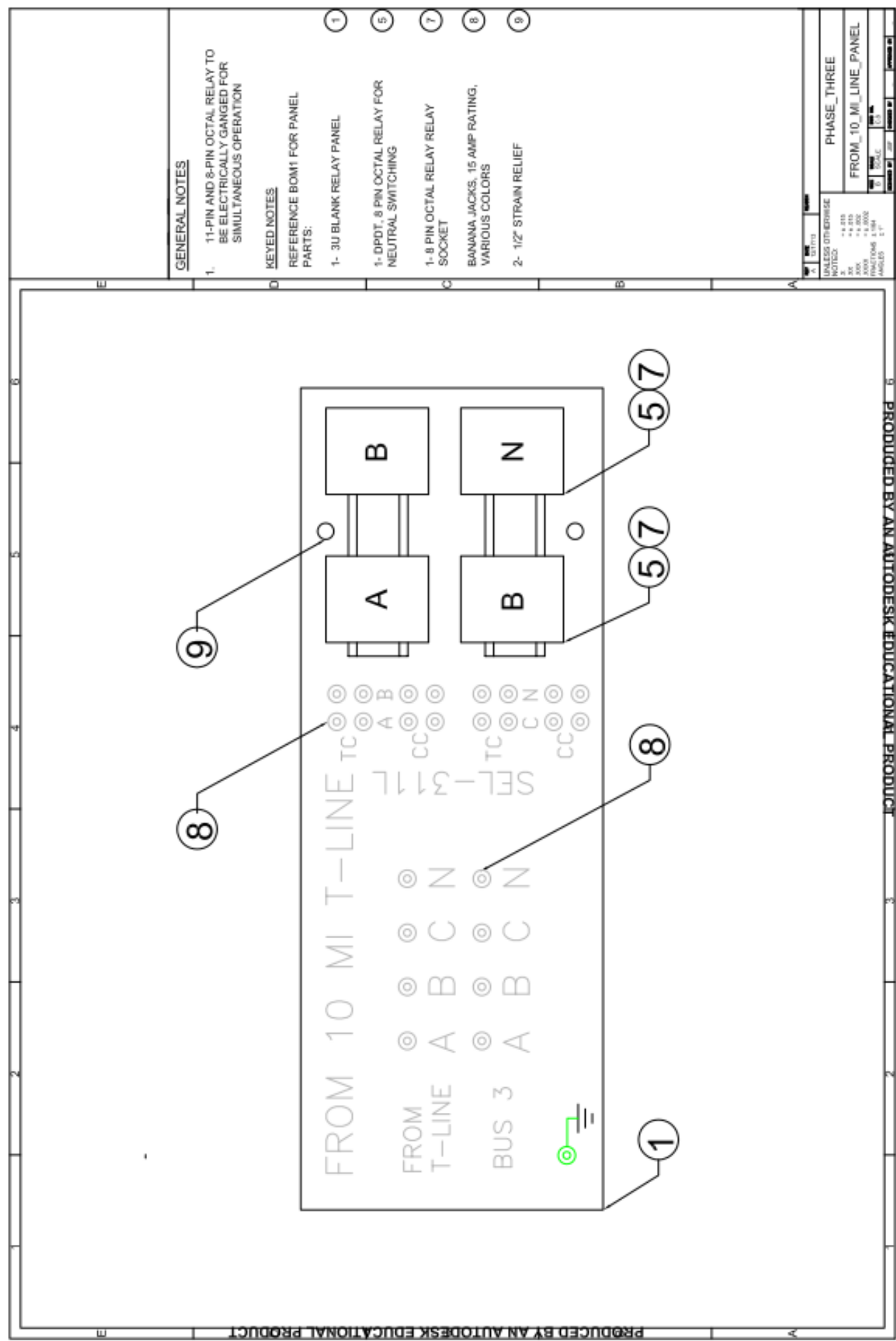


D.23 Phase III model-scale power system To 10 Mile Line panel drawing

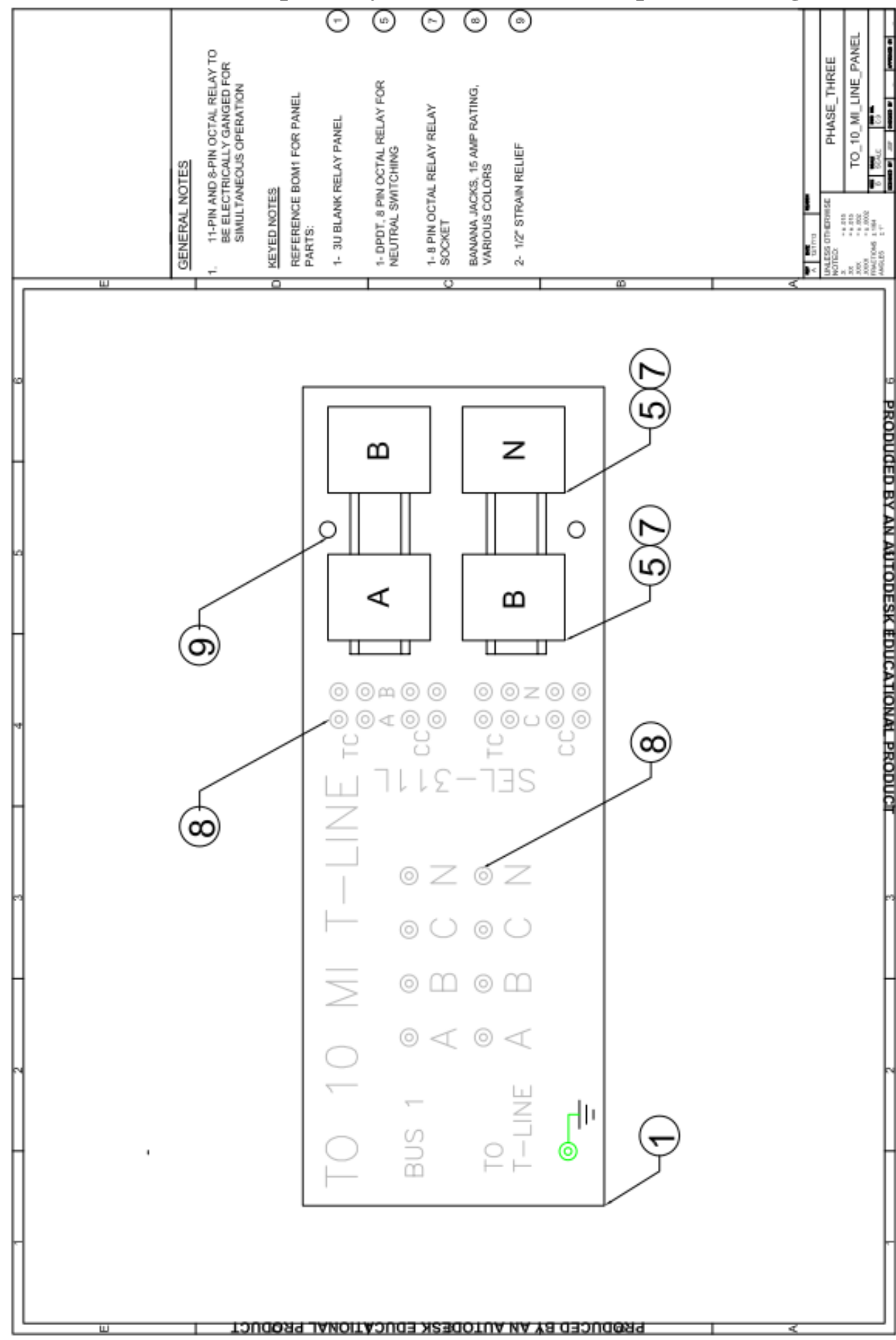


D.24 Phase III model-scale power system From 8 Mile Line panel drawing

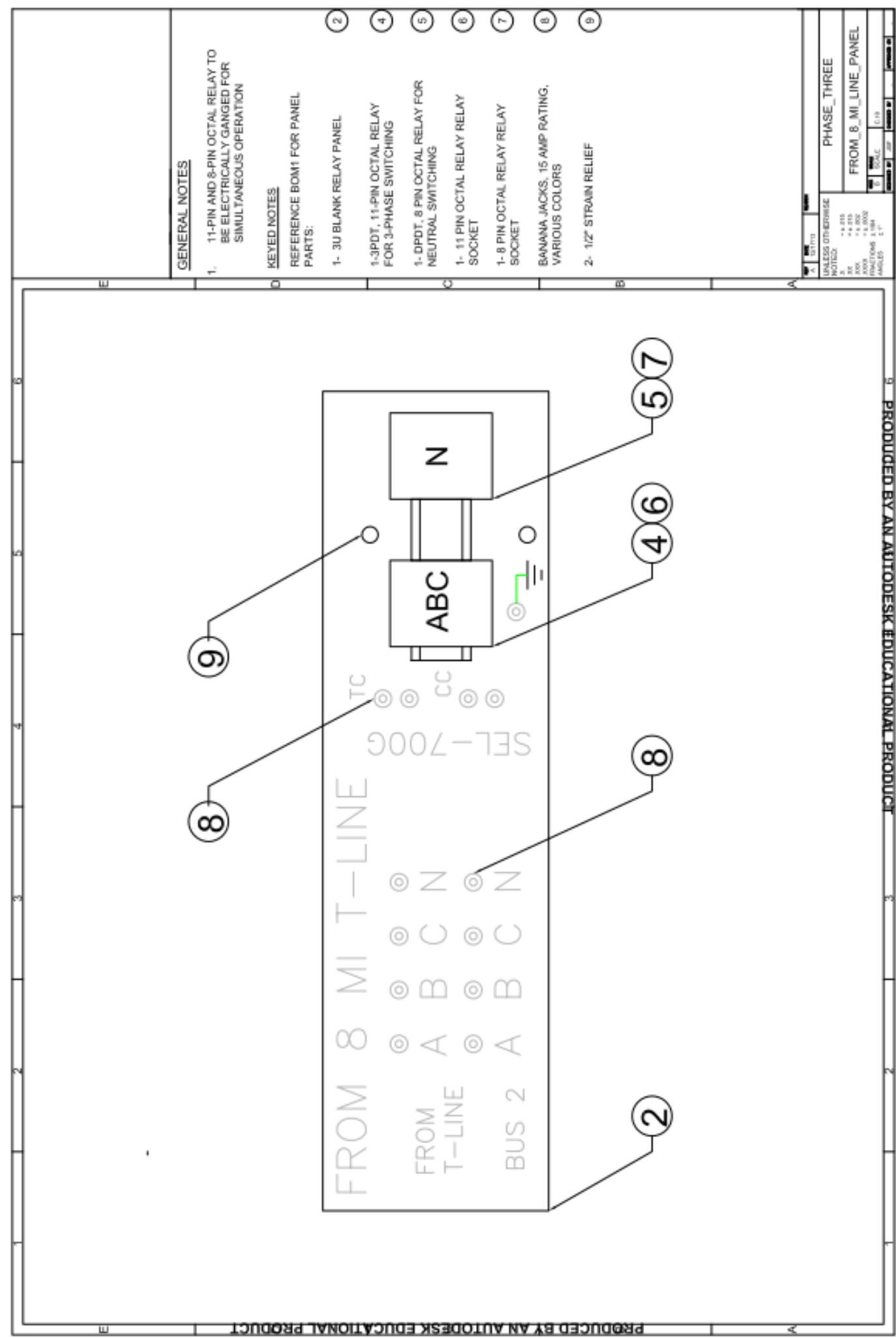



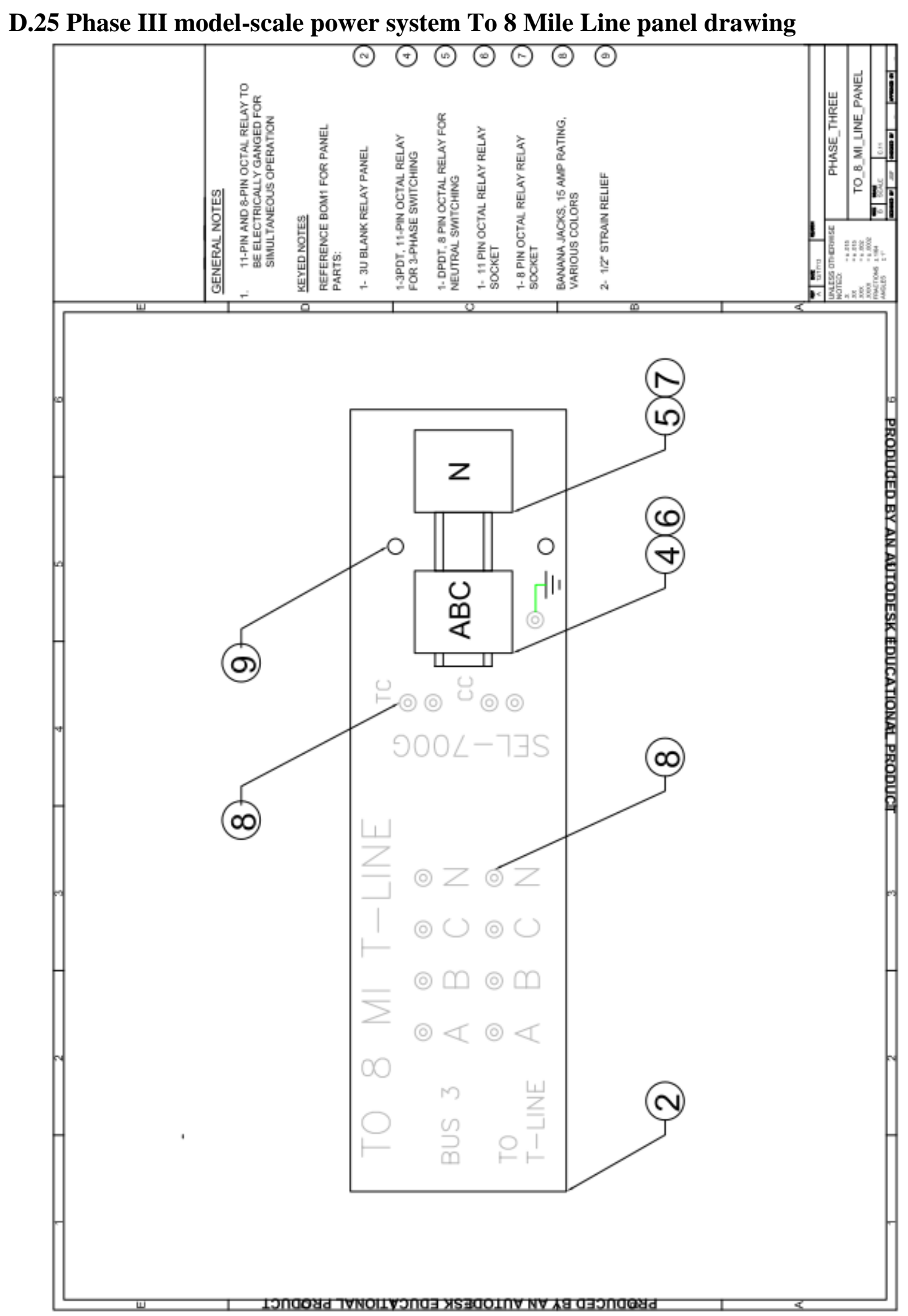
D.26 Phase III model-scale power system From 6 Mile Line panel drawing
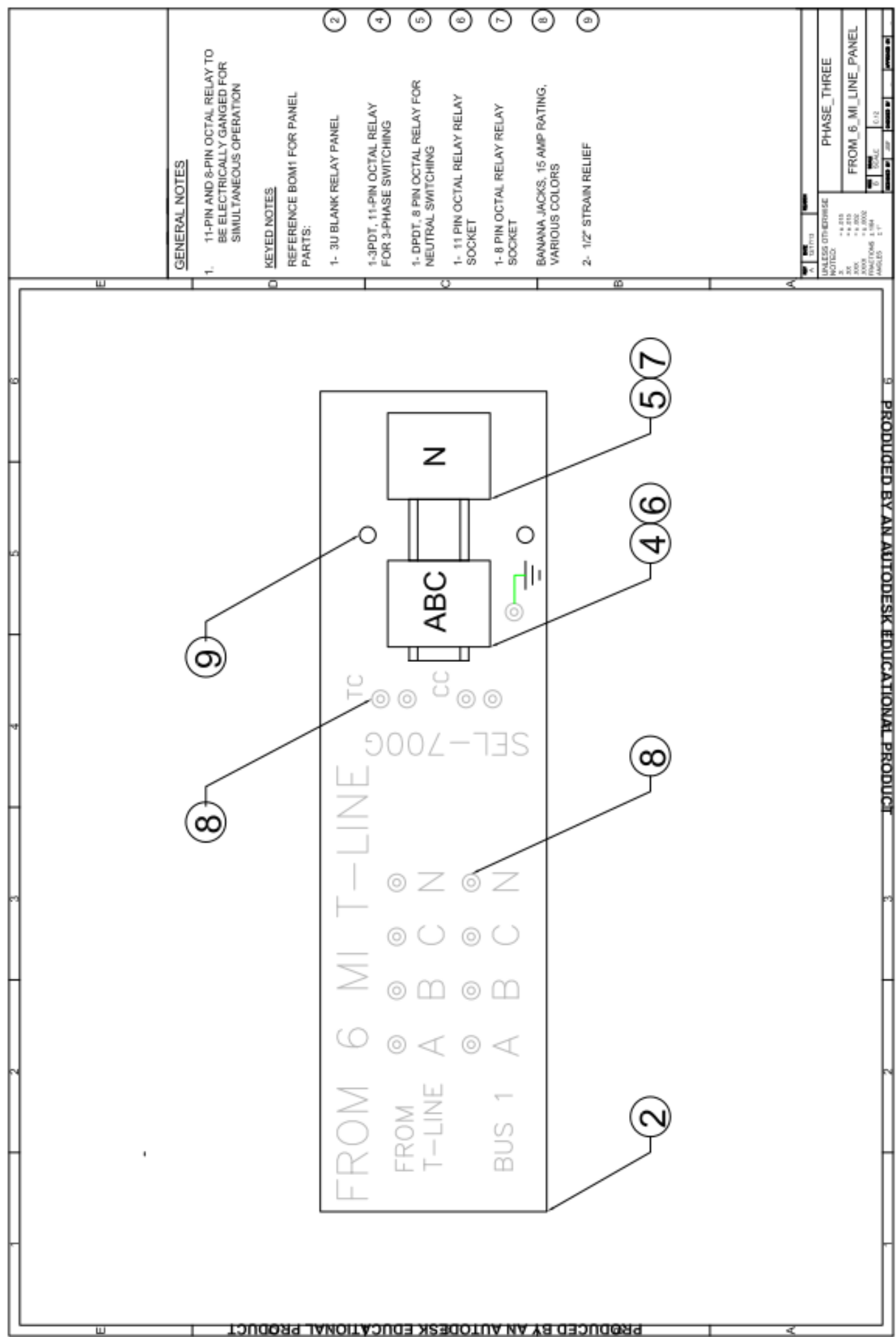


\section{D.27 Phase III model-scale power system To 6 Mile Line panel drawing}

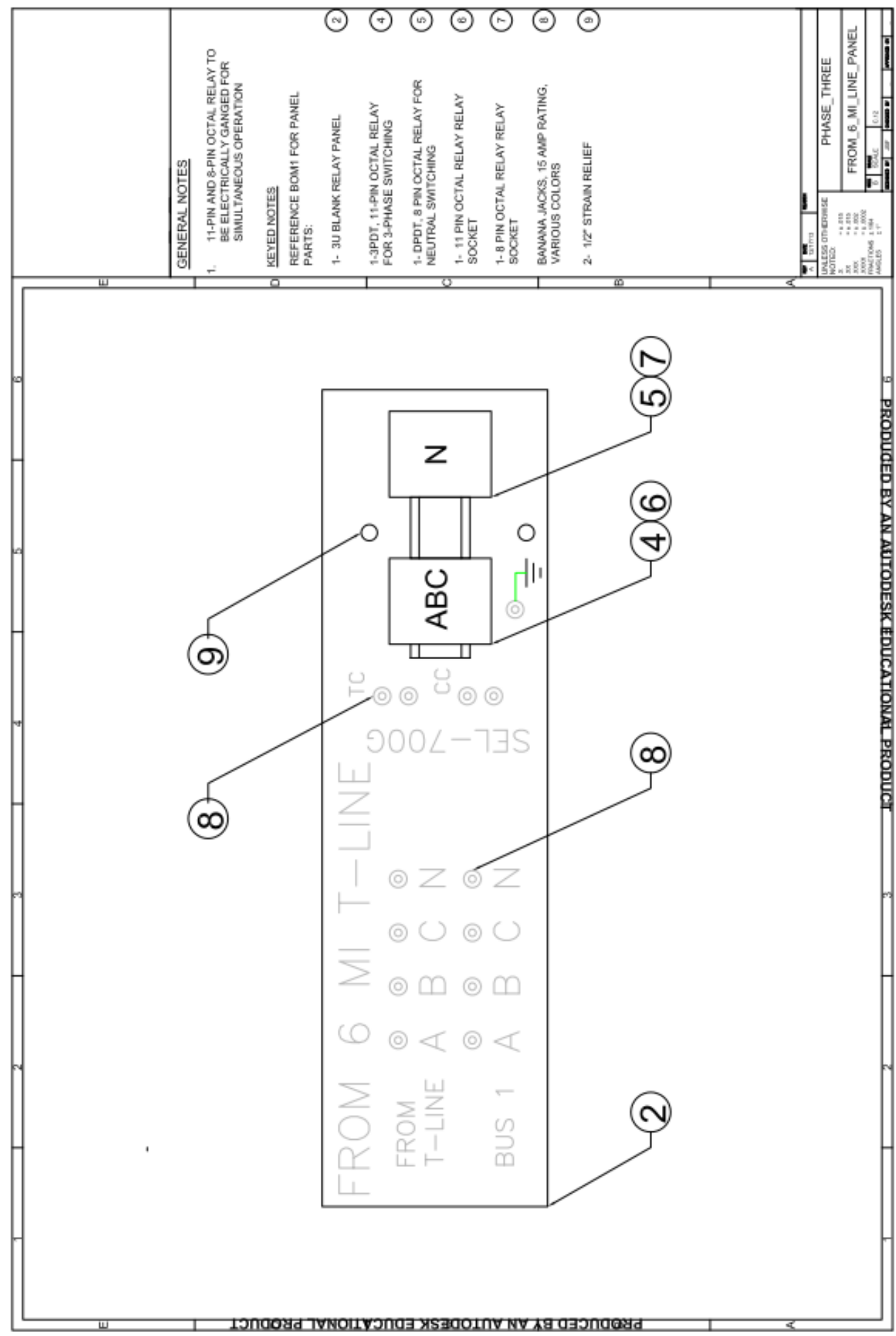



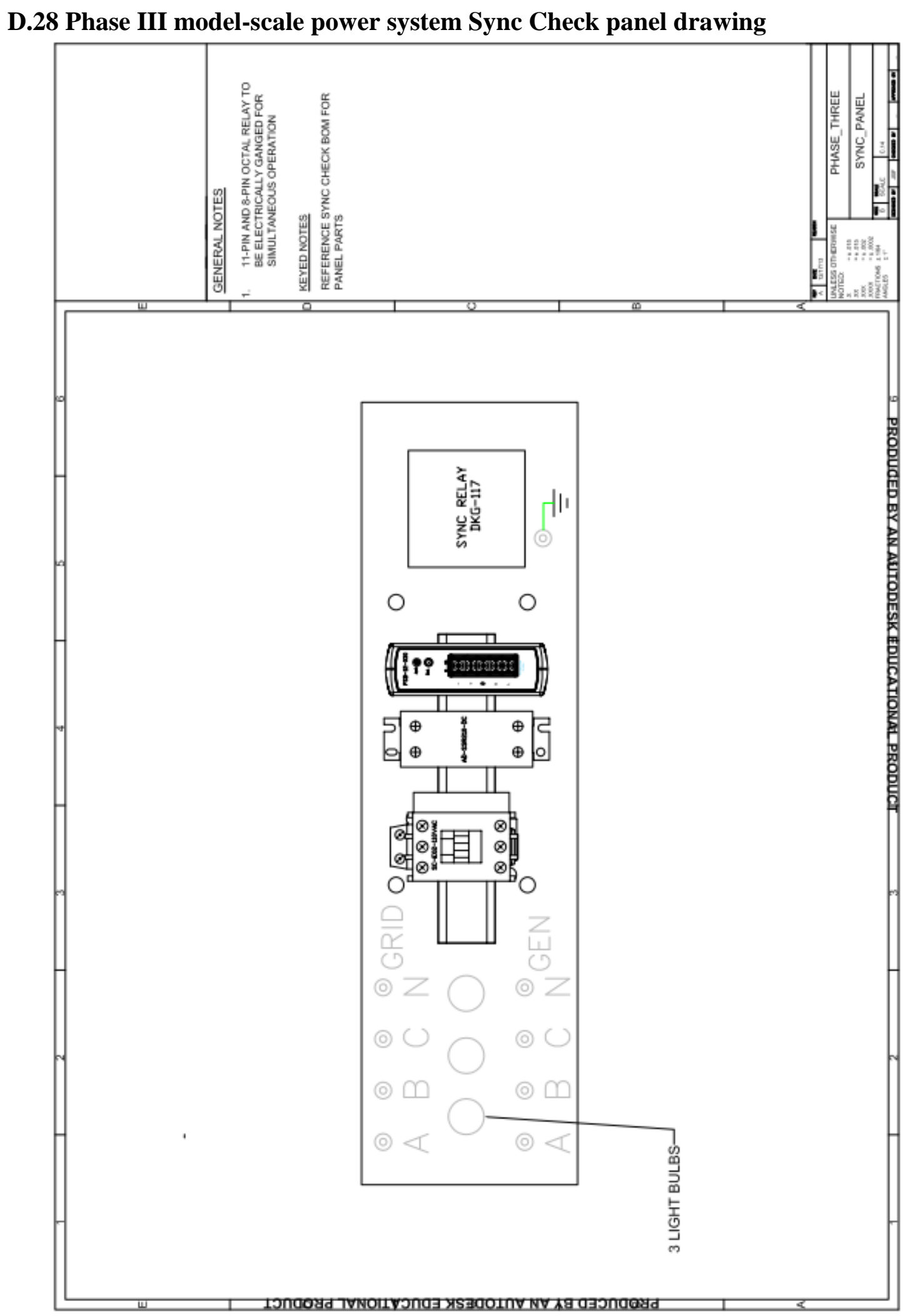
Appendix E: Laboratory Assignment Rubrics 


\title{
E.1 ASPEN Software Introduction Rubric
}

\author{
Portland State University \\ Electrical \& Computer Engineering \\ ECE 448/548 Power System Protection I \\ -Lab 1: ASPEN Introduction-
}

TA:

STUDENT NAMES:

GRADE

\begin{abstract}
ABET/RES Program Outcomes
(a) an ability to apply knowledge of mathematics, science, and engineering

(b) an ability to design and conduct experiments, as well as to analyze and interpret data

(d) an ability to function on multi-disciplinary teams

(g) an ability to communicate effectively
\end{abstract}

\begin{tabular}{|c|c|c|c|c|}
\hline CRITERIA & 1-DEVELOPING & 2-ACCOMPLISHED & 3-EXEMPLARY & SCORE \\
\hline $\begin{array}{l}\text { ASSIGNED } \\
\text { TASKS } \\
\text { Outcomes: b }\end{array}$ & $\begin{array}{l}\text { Did not perform, or did } \\
\text { not show an } \\
\text { understanding of, the } \\
\text { following tasks: } \\
\text { 8) Model radial and } \\
\text { looped power systems } \\
\text { using ASPEN software; } \\
\text { 2) Simulate systems } \\
\text { using ASPEN software; } \\
\text { 3) Perform basic power } \\
\text { studies and properly } \\
\text { interpret results; 4) } \\
\text { Perform fault studies } \\
\text { and properly interpret } \\
\text { results; 5) Correctly } \\
\text { calculate per unit } \\
\text { values; 6) Overall, } \\
\text { assigned tasks were not } \\
\text { performed competently. }\end{array}$ & $\begin{array}{l}\text { Performed and discussed } \\
\text { the following tasks: } \\
\text { 1) Model radial and } \\
\text { looped power systems } \\
\text { using ASPEN software; } \\
\text { 2) Simulate systems } \\
\text { using ASPEN software; } \\
\text { 3) Perform basic power } \\
\text { studies and properly } \\
\text { interpret results; 4) } \\
\text { Perform fault studies and } \\
\text { properly interpret } \\
\text { results; 5) Correctly } \\
\text { calculate per unit values; } \\
\text { 6) Overall, assigned } \\
\text { tasks were performed } \\
\text { competently. }\end{array}$ & $\begin{array}{l}\text { Demonstrated excellent } \\
\text { understanding of the } \\
\text { following tasks: } \\
\text { 1) Model radial and } \\
\text { looped power systems } \\
\text { using ASPEN software; } \\
\text { 2) Simulate systems } \\
\text { using ASPEN software; } \\
\text { 3) Perform basic power } \\
\text { studies and analysis on } \\
\text { system model } \\
\text { and properly interpret } \\
\text { results; 4) Perform fault } \\
\text { studies and properly } \\
\text { interpret results; 5) } \\
\text { Correctly calculate per } \\
\text { unit values; 6) Overall, } \\
\text { assigned tasks were } \\
\text { performed competently } \\
\text { and additional } \\
\text { investigations also } \\
\text { conducted. }\end{array}$ & \\
\hline $\begin{array}{l}\text { TEAM WORK } \\
\text { Outcomes: d }\end{array}$ & $\begin{array}{l}\text { 1) Group does not } \\
\text { function effective as a } \\
\text { team, group dissonance } \\
\text { is apparent.; 2) } \\
\text { members do not } \\
\text { contribute significantly } \\
\text { to the team; 3) group } \\
\text { does not delegate } \\
\text { responsibilities or does } \\
\text { so poorly. }\end{array}$ & $\begin{array}{l}\text { 1) Group functions as a } \\
\text { team; 2) members } \\
\text { assume roles as } \\
\text { individual contributors, } \\
\text { moderately enhancing } \\
\text { the team performance; } 3 \text { ) } \\
\text { group effectively } \\
\text { delegates responsibilities } \\
\text { to manage the project. }\end{array}$ & $\begin{array}{l}\text { 1) Group functions } \\
\text { effectively as a team; } 2 \text { ) } \\
\text { members assume roles as } \\
\text { individual contributors, } \\
\text { significantly enhancing } \\
\text { the team performance; } 3 \text { ) } \\
\text { effectively delegate } \\
\text { responsibilities, } \\
\text { leveraging individual } \\
\text { expertise, interests \& } \\
\text { skills. }\end{array}$ & \\
\hline
\end{tabular}




\begin{tabular}{|c|c|c|c|c|}
\hline $\begin{array}{l}\text { Data ANalysis } \\
\text { \& } \\
\text { Presentation } \\
\text { Outcomes: } \\
\text { a, b, g }\end{array}$ & $\begin{array}{l}\text { Tables and figures, if } \\
\text { included, 1) are not } \\
\text { numbered, 2) lack } \\
\text { captions, 3) axis not } \\
\text { labeled; 4) data, if } \\
\text { presented, is neither } \\
\text { analyzed nor 5) } \\
\text { properly interpreted; 6) } \\
\text { do not apply } \\
\text { engineering knowledge } \\
\text { in the analysis of data; } \\
\text { 7) report is too } \\
\text { long/short; not well } \\
\text { written. }\end{array}$ & $\begin{array}{l}\text { 1) Figures and tables } \\
\text { enhance the report, and } \\
\text { 2) are numbered, 3) } \\
\text { though may lack } \\
\text { descriptive captions, or } \\
\text { 4) have incomplete axis } \\
\text { labels; 5) data presented } \\
\text { is analyzed and 6) } \\
\text { properly interpreted; 7) } \\
\text { apply engineering } \\
\text { knowledge in analysis of } \\
\text { data; 8) report is concise } \\
\text { and well written. }\end{array}$ & $\begin{array}{l}\text { 1) High quality figures } \\
\text { and tables add depth to } \\
\text { the report, 2) are } \\
\text { numbered, and 3) include } \\
\text { descriptive captions and } \\
\text { 4) well labeled axis; 5) } \\
\text { data presented is clearly } \\
\text { analyzed and 6) expertly } \\
\text { interpreted; 7) clearly } \\
\text { apply engineering } \\
\text { knowledge in analysis of } \\
\text { data; 8) report is concise } \\
\text { and exceptionally well } \\
\text { written. }\end{array}$ & 5 \\
\hline
\end{tabular}

Adopted from rubrics made available by Professor Mary Mueller of Seton Hall University as well as Professor Bob Bass of Portland State University. 


\title{
E.2 Wire Heating and Fuses Rubric
}

\author{
Portland State University \\ Electrical \& Computer Engineering \\ ECE 448/548 Power System Protection I \\ -Lab 2: Wire Heating and Fuses-
}

TA:

GRADE

STUDENT NAMES:

\begin{abstract}
ABET/RES Program Outcomes
(a) an ability to apply knowledge of mathematics, science, and engineering

(b) an ability to design and conduct experiments, as well as to analyze and interpret data

(d) an ability to function on multi-disciplinary teams

(g) an ability to communicate effectively
\end{abstract}

\begin{tabular}{|c|c|c|c|c|}
\hline CRITERIA & 1-DEVELOPING & 2-ACCOMPLISHED & 3-EXEMPLARY & SCORE \\
\hline $\begin{array}{l}\text { ASSIGNED } \\
\text { TASKS } \\
\text { Outcomes: b }\end{array}$ & $\begin{array}{l}\text { Did not perform, or did } \\
\text { not show an } \\
\text { understanding of, the } \\
\text { following tasks: } \\
\text { Plot free air time- } \\
\text { temperature curve } \\
\text { using experimental } \\
\text { data at two different } \\
\text { current levels; 2) } \\
\text { Model free-air time - } \\
\text { temperature curve in } \\
\text { Matlab and compare to } \\
\text { experimental data; } 3 \text { ) } \\
\text { Plot approximate } \\
\text { time-current melting } \\
\text { curve for a conductor; } \\
\text { 4) Verify conductor } \\
\text { material type based on } \\
\text { thermal behavior; } \\
\text { 5) Overall, assigned } \\
\text { tasks were not } \\
\text { performed } \\
\text { competently. }\end{array}$ & $\begin{array}{l}\text { Performed and discussed } \\
\text { the following tasks: } \\
\text { 1) Plot free air time- } \\
\text { temperature curve using } \\
\text { experimental data at two } \\
\text { different current levels; } \\
\text { 2) Model free-air time - } \\
\text { temperature curve in } \\
\text { Matlab and compare to } \\
\text { experimental data; 3) } \\
\text { Plot approximate time- } \\
\text { current melting curve for } \\
\text { a conductor; 4) Verify } \\
\text { conductor material type } \\
\text { based on thermal } \\
\text { behavior; 5) Overall, } \\
\text { assigned tasks were } \\
\text { performed competently. }\end{array}$ & $\begin{array}{l}\text { Demonstrated excellent } \\
\text { understanding of the } \\
\text { following tasks: } \\
\text { 1) Plot free air time- } \\
\text { temperature curve using } \\
\text { experimental data at two } \\
\text { different current levels; 2) } \\
\text { Model free-air time - } \\
\text { temperature curve in } \\
\text { Matlab and compare to } \\
\text { experimental data; } 3 \text { ) } \\
\text { Plot approximate time- } \\
\text { current melting curve for } \\
\text { a conductor; 4) Verify } \\
\text { conductor material type } \\
\text { based on thermal } \\
\text { behavior; 5) Overall, } \\
\text { assigned tasks were } \\
\text { performed competently } \\
\text { and additional } \\
\text { investigations also } \\
\text { conducted. }\end{array}$ & \\
\hline $\begin{array}{l}\text { TEAM WORK } \\
\text { Outcomes: d }\end{array}$ & $\begin{array}{l}\text { 1) Group does not } \\
\text { function effective as a } \\
\text { team, group } \\
\text { dissonance is } \\
\text { apparent.; 2) members } \\
\text { do not contribute } \\
\text { significantly to the } \\
\text { team; 3) group does } \\
\text { not delegate } \\
\text { responsibilities or does } \\
\text { so poorly. }\end{array}$ & $\begin{array}{l}\text { 1) Group functions as a } \\
\text { team; 2) members } \\
\text { assume roles as } \\
\text { individual contributors, } \\
\text { moderately enhancing } \\
\text { the team performance; } 3 \text { ) } \\
\text { group effectively } \\
\text { delegates responsibilities } \\
\text { to manage the project. }\end{array}$ & $\begin{array}{l}\text { 1) Group functions } \\
\text { effectively as a team; 2) } \\
\text { members assume roles as } \\
\text { individual contributors, } \\
\text { significantly enhancing } \\
\text { the team performance; } 3 \text { ) } \\
\text { effectively delegate } \\
\text { responsibilities, } \\
\text { leveraging individual } \\
\text { expertise, interests \& } \\
\text { skills. }\end{array}$ & \\
\hline
\end{tabular}




\begin{tabular}{|c|c|c|c|c|}
\hline $\begin{array}{l}\text { DAta ANAlysis } \\
\text { \& } \\
\text { PreSENTAtion } \\
\text { Outcomes: } \\
\text { a, b, g }\end{array}$ & $\begin{array}{l}\text { Tables and figures, if } \\
\text { included, 1) are not } \\
\text { numbered, 2) lack } \\
\text { captions, 3) axis not } \\
\text { labeled; 4) data, if } \\
\text { presented, is neither } \\
\text { analyzed nor 5) } \\
\text { properly interpreted; 6) } \\
\text { do not apply } \\
\text { engineering knowledge } \\
\text { in the analysis of data; } \\
\text { 7) report is too } \\
\text { long/short; not well } \\
\text { written. }\end{array}$ & $\begin{array}{l}\text { 1) Figures and tables } \\
\text { enhance the report, and } \\
\text { 2) are numbered, 3) } \\
\text { though may lack } \\
\text { descriptive captions, or } \\
\text { 4) have incomplete axis } \\
\text { labels; 5) data presented } \\
\text { is analyzed and 6) } \\
\text { properly interpreted; 7) } \\
\text { apply engineering } \\
\text { knowledge in analysis of } \\
\text { data; 8) report is concise } \\
\text { and well written. }\end{array}$ & $\begin{array}{l}\text { 1) High quality figures } \\
\text { and tables add depth to } \\
\text { the report, 2) are } \\
\text { numbered, and 3) include } \\
\text { descriptive captions and } \\
\text { 4) well labeled axis; 5) } \\
\text { data presented is clearly } \\
\text { analyzed and 6) expertly } \\
\text { interpreted. 7) clearly } \\
\text { apply engineering } \\
\text { knowledge in analysis of } \\
\text { data; 8) report is concise } \\
\text { and exceptionally well } \\
\text { written. }\end{array}$ & $/ 5$ \\
\hline
\end{tabular}

Adopted from rubrics made available by Professor Mary Mueller of Seton Hall University as well as Professor Bob Bass of Portland State University. 


\title{
E.3 Auxiliary Relay Testing Rubric
}

\author{
Portland State University \\ Electrical \& Computer Engineering \\ ECE 448/548 Power System Protection I \\ -Lab 3: Auxiliary Relay Testing -
}

TA:

STUDENT NAMES:

GRADE

\begin{abstract}
ABET/RES Program Outcomes
(a) an ability to apply knowledge of mathematics, science, and engineering

(b) an ability to design and conduct experiments, as well as to analyze and interpret data

(d) an ability to function on multi-disciplinary teams

(g) an ability to communicate effectively
\end{abstract}

\begin{tabular}{|c|c|c|c|c|}
\hline CRITERIA & 1-DEVELOPING & 2-ACCOMPLISHED & 3-EXEMPLARY & SCORE \\
\hline $\begin{array}{l}\text { ASSIGNED } \\
\text { TASKS } \\
\text { Outcomes: b }\end{array}$ & $\begin{array}{l}\text { Did not perform, or } \\
\text { did not show an } \\
\text { understanding of, the } \\
\text { following tasks: } \\
\text { Plot free air time- } \\
\text { temperature curve } \\
\text { using experimental } \\
\text { data at two different } \\
\text { current levels; 2) } \\
\text { Model free-air time - } \\
\text { temperature curve in } \\
\text { Matlab and compare to } \\
\text { experimental data; } 3 \text { ) } \\
\text { Plot approximate } \\
\text { time-current melting } \\
\text { curve for a conductor; } \\
\text { 4) Verify conductor } \\
\text { material type based on } \\
\text { thermal behavior; 5) } \\
\text { Overall, assigned tasks } \\
\text { were not performed } \\
\text { competently. }\end{array}$ & $\begin{array}{l}\text { Performed and discussed } \\
\text { the following tasks: } \\
\text { 1) Plot free air time- } \\
\text { temperature curve using } \\
\text { experimental data at two } \\
\text { different current levels; } \\
\text { 2) Model free-air time - } \\
\text { temperature curve in } \\
\text { Matlab and compare to } \\
\text { experimental data; 3) } \\
\text { Plot approximate time- } \\
\text { current melting curve for } \\
\text { a conductor; 4) Verify } \\
\text { conductor material type } \\
\text { based on thermal } \\
\text { behavior; 5) Overall, } \\
\text { assigned tasks were } \\
\text { performed competently. }\end{array}$ & $\begin{array}{l}\text { Demonstrated excellent } \\
\text { understanding of the } \\
\text { following tasks: } \\
\text { 1) Plot free air time- } \\
\text { temperature curve using } \\
\text { experimental data at two } \\
\text { different current levels; } \\
\text { 2) Model free-air time - } \\
\text { temperature curve in } \\
\text { Matlab and compare to } \\
\text { experimental data; 3) } \\
\text { Plot approximate time- } \\
\text { current melting curve for } \\
\text { a conductor; 4) Verify } \\
\text { conductor material type } \\
\text { based on thermal } \\
\text { behavior; 5) Overall, } \\
\text { assigned tasks were } \\
\text { performed competently } \\
\text { and additional } \\
\text { investigations also } \\
\text { conducted. }\end{array}$ & \\
\hline $\begin{array}{l}\text { TEAM WORK } \\
\text { Outcomes: d }\end{array}$ & $\begin{array}{l}\text { 1) Group does not } \\
\text { function effective as a } \\
\text { team, group } \\
\text { dissonance is } \\
\text { apparent.; 2) members } \\
\text { do not contribute } \\
\text { significantly to the } \\
\text { team; 3) group does } \\
\text { not delegate } \\
\text { responsibilities or does } \\
\text { so poorly. }\end{array}$ & $\begin{array}{l}\text { 1) Group functions as a } \\
\text { team; 2) members } \\
\text { assume roles as } \\
\text { individual contributors, } \\
\text { moderately enhancing } \\
\text { the team performance; } 3 \text { ) } \\
\text { group effectively } \\
\text { delegates responsibilities } \\
\text { to manage the project. }\end{array}$ & $\begin{array}{l}\text { 1) Group functions } \\
\text { effectively as a team; 2) } \\
\text { members assume roles as } \\
\text { individual contributors, } \\
\text { significantly enhancing } \\
\text { the team performance; 3) } \\
\text { effectively delegate } \\
\text { responsibilities, } \\
\text { leveraging individual } \\
\text { expertise, interests \& } \\
\text { skills. }\end{array}$ & \\
\hline
\end{tabular}




\begin{tabular}{|c|c|c|c|c|}
\hline $\begin{array}{l}\text { Data ANalysis } \\
\text { \& } \\
\text { Presentation } \\
\text { Outcomes: } \\
\text { a, b, g }\end{array}$ & $\begin{array}{l}\text { Tables and figures, if } \\
\text { included, 1) are not } \\
\text { numbered, 2) lack } \\
\text { captions, 3) axis not } \\
\text { labeled; 4) data, if } \\
\text { presented, is neither } \\
\text { analyzed nor 5) } \\
\text { properly interpreted; } \\
\text { 6) do not apply } \\
\text { engineering } \\
\text { knowledge in the } \\
\text { analysis of data; 7) } \\
\text { report is too } \\
\text { long/short; not well } \\
\text { written. }\end{array}$ & $\begin{array}{l}\text { 1) Figures and tables } \\
\text { enhance the report, and } \\
\text { 2) are numbered, 3) } \\
\text { though may lack } \\
\text { descriptive captions, or } \\
\text { 4) have incomplete axis } \\
\text { labels; 5) data presented } \\
\text { is analyzed and 6) } \\
\text { properly interpreted; 7) } \\
\text { apply engineering } \\
\text { knowledge in analysis of } \\
\text { data; 8) report is concise } \\
\text { and well written. }\end{array}$ & $\begin{array}{l}\text { 1) High quality figures } \\
\text { and tables add depth to } \\
\text { the report, 2) are } \\
\text { numbered, and 3) include } \\
\text { descriptive captions and } \\
\text { 4) well labeled axis; 5) } \\
\text { data presented is clearly } \\
\text { analyzed and 6) expertly } \\
\text { interpreted. 7) clearly } \\
\text { apply engineering } \\
\text { knowledge in analysis of } \\
\text { data; 8) report is concise } \\
\text { and exceptionally well } \\
\text { written. }\end{array}$ & 5 \\
\hline
\end{tabular}

Adopted from rubrics made available by Professor Mary Mueller of Seton Hall University as well as Professor Bob Bass of Portland State University. 


\title{
E.4 Current Transformer Testing Rubric
}

\author{
Portland State University \\ Electrical \& Computer Engineering \\ ECE 448/548 Power System Protection I \\ -Lab 4: Current Transformer Testing-
}

TA:

GRADE

STUDENT NAMES:

\begin{tabular}{|c|c|c|c|c|}
\hline \multicolumn{5}{|c|}{$\begin{array}{l}\text { ABET/RES Program Outcomes } \\
\text { (a) an ability to apply knowledge of mathematics, science, and engineering } \\
\text { (b) an ability to design and conduct experiments, as well as to analyze and interpret data } \\
\text { (d) an ability to function on multi-disciplinary teams } \\
\text { (g) an ability to communicate effectively }\end{array}$} \\
\hline CRITERIA & 1-DEVELOPING & 2-ACCOMPLISHED & 3-EXEMPLARY & SCORE \\
\hline $\begin{array}{l}\text { ASSIGNED } \\
\text { TASKS } \\
\text { Outcomes: b }\end{array}$ & $\begin{array}{l}\text { Did not perform, or did } \\
\text { not show an } \\
\text { understanding of, the } \\
\text { following tasks: } \\
\text { 1) Determining CT } \\
\text { accuracy class and } \\
\text { burden rating; } \\
\text { 2) Constructing the } \\
\text { magnetization curves of } \\
\text { different CT ratios; } \\
\text { 3) Experimentally } \\
\text { determining CT burden } \\
\text { through magnetization } \\
\text { curve analysis; 4) } \\
\text { Comparing the } \\
\text { experimental results } \\
\text { with the burden rating } \\
\text { given by the } \\
\text { manufacture in } \\
\text { the CT datasheet; 5) } \\
\text { Overall, assigned tasks } \\
\text { were not performed } \\
\text { competently. }\end{array}$ & $\begin{array}{l}\text { Performed and } \\
\text { discussed the following } \\
\text { tasks: } \\
\text { 1) Determining CT } \\
\text { accuracy class and } \\
\text { burden rating; } \\
\text { 2) Constructing the } \\
\text { magnetization curves of } \\
\text { different CT ratios; } \\
\text { 3) Experimentally } \\
\text { determining CT burden } \\
\text { through magnetization } \\
\text { curve analysis; 4) } \\
\text { Comparing the } \\
\text { experimental results } \\
\text { with the burden rating } \\
\text { given by the } \\
\text { manufacture in } \\
\text { the CT datasheet; 5) } \\
\text { Overall, assigned tasks } \\
\text { were performed } \\
\text { competently. }\end{array}$ & $\begin{array}{l}\text { Demonstrated excellent } \\
\text { understanding of the } \\
\text { following tasks: } \\
\text { 1) Determining CT } \\
\text { accuracy class and burden } \\
\text { rating; } \\
\text { 2) Constructing the } \\
\text { magnetization curves of } \\
\text { different CT ratios; } \\
\text { 3) Experimentally } \\
\text { determining CT burden } \\
\text { through magnetization } \\
\text { curve analysis; 4) } \\
\text { Comparing the } \\
\text { experimental results with } \\
\text { the burden rating given } \\
\text { by the manufacture in } \\
\text { the CT datasheet; 5) } \\
\text { Overall, assigned tasks } \\
\text { were performed } \\
\text { competently and } \\
\text { additional investigations } \\
\text { also conducted. }\end{array}$ & \\
\hline $\begin{array}{l}\text { TEAM WORK } \\
\text { Outcomes: d }\end{array}$ & $\begin{array}{l}\text { 1) Group does not } \\
\text { function effective as a } \\
\text { team, group dissonance } \\
\text { is apparent.; 2) } \\
\text { members do not } \\
\text { contribute significantly } \\
\text { to the team; 3) group } \\
\text { does not delegate } \\
\text { responsibilities or does } \\
\text { so poorly. }\end{array}$ & $\begin{array}{l}\text { 1) Group functions as a } \\
\text { team; 2) members } \\
\text { assume roles as } \\
\text { individual contributors, } \\
\text { moderately enhancing } \\
\text { the team performance; } \\
\text { 3) group effectively } \\
\text { delegates } \\
\text { responsibilities to } \\
\text { manage the project. }\end{array}$ & $\begin{array}{l}\text { 1) Group functions } \\
\text { effectively as a team; 2) } \\
\text { members assume roles as } \\
\text { individual contributors, } \\
\text { significantly enhancing } \\
\text { the team performance; } 3 \text { ) } \\
\text { effectively delegate } \\
\text { responsibilities, } \\
\text { leveraging individual } \\
\text { expertise, interests \& } \\
\text { skills. }\end{array}$ & \\
\hline
\end{tabular}




\begin{tabular}{|c|c|c|c|c|}
\hline $\begin{array}{l}\text { Data ANalysis } \\
\text { \& } \\
\text { Presentation } \\
\text { Outcomes: } \\
\text { a, b, g }\end{array}$ & $\begin{array}{l}\text { Tables and figures, if } \\
\text { included, 1) are not } \\
\text { numbered, 2) lack } \\
\text { captions, 3) axis not } \\
\text { labeled; 4) data, if } \\
\text { presented, is neither } \\
\text { analyzed nor 5) } \\
\text { properly interpreted; 6) } \\
\text { do not apply } \\
\text { engineering knowledge } \\
\text { in the analysis of data; } \\
\text { 7) report is too } \\
\text { long/short; not well } \\
\text { written. }\end{array}$ & $\begin{array}{l}\text { Figures and tables } \\
\text { enhance the report, and } \\
\text { 1) are numbered, 2) } \\
\text { though may lack } \\
\text { descriptive captions, or } \\
\text { 3) have incomplete axis } \\
\text { labels; 4) data presented } \\
\text { is analyzed and 5) } \\
\text { properly interpreted; 6) } \\
\text { apply engineering } \\
\text { knowledge in analysis } \\
\text { of data; 7) report is } \\
\text { concise and well } \\
\text { written. }\end{array}$ & $\begin{array}{l}\text { High quality figures and } \\
\text { tables add depth to the } \\
\text { report, 1) are numbered, } \\
\text { and 2) include descriptive } \\
\text { captions and 3) well } \\
\text { labeled axis; 4) data } \\
\text { presented is clearly } \\
\text { analyzed and 5) expertly } \\
\text { interpreted. 6) clearly } \\
\text { apply engineering } \\
\text { knowledge in analysis of } \\
\text { data; 7) report is concise } \\
\text { and exceptionally well } \\
\text { written. }\end{array}$ & $/ 5$ \\
\hline
\end{tabular}

Adopted from rubrics made available by Professor Mary Mueller of Seton Hall University as well as Professor Bob Bass of Portland State University. 


\section{E.5 EM Relay Radial Over-current Protection (50/51) Rubric Portland State University Electrical \& Computer Engineering ECE 448/548 Power System Protection I -Lab 5: EM Relay Radial Over-current Protection (50/51)-}

TA: GRADE

\section{STUDENT NAMES:
ABET/RES Program Outcomes}

(a) an ability to apply knowledge of mathematics, science, and engineering

(b) an ability to design and conduct experiments, as well as to analyze and interpret data

(d) an ability to function on multi-disciplinary teams

(g) an ability to communicate effectively

\begin{tabular}{|c|c|c|c|c|}
\hline CRITERIA & 1-DEVELOPING & 2-ACCOMPLISHED & 3-EXEMPLARY & SCORE \\
\hline $\begin{array}{l}\text { ASSIGNED } \\
\text { TASKS } \\
\text { Outcomes: b }\end{array}$ & $\begin{array}{l}\text { Did not perform, or did } \\
\text { not show an } \\
\text { understanding of, the } \\
\text { following tasks: } \\
\text { 1) Identifying the most } \\
\text { important part of an } \\
\text { electromechanical over- } \\
\text { current relay; } \\
\text { 2) Explaining the } \\
\text { application of each part } \\
\text { of the relay; } \\
\text { 3) Determining the pick- } \\
\text { up current of the } \\
\text { inverse-time relay } \\
\text { element using a relay } \\
\text { testing equipment; } 4 \text { ) } \\
\text { Constructing the time- } \\
\text { current curve of the EM } \\
\text { relay; 5) Determining } \\
\text { the instantaneous current } \\
\text { of the inverse-time relay } \\
\text { element using relay } \\
\text { testing equipment; } \\
\text { 6) Comparing the results } \\
\text { with the characteristics } \\
\text { given by the } \\
\text { manufacture in the } \\
\text { relay's } \\
\text { manual; 7) Overall, } \\
\text { assigned tasks were not } \\
\text { performed competently. }\end{array}$ & $\begin{array}{l}\text { Performed and } \\
\text { discussed the following } \\
\text { tasks: } \\
\text { 1) Identifying the most } \\
\text { important part of an } \\
\text { electromechanical over- } \\
\text { current relay; } \\
\text { 2) Explaining the } \\
\text { application of each part } \\
\text { of the relay; } \\
\text { 3) Determining the } \\
\text { pick-up current of the } \\
\text { inverse-time relay } \\
\text { element using a relay } \\
\text { testing equipment; } 4) \\
\text { Constructing the time- } \\
\text { current curve of the EM } \\
\text { relay; 5) Determining } \\
\text { the instantaneous } \\
\text { current of the inverse- } \\
\text { time relay element using } \\
\text { relay testing equipment; } \\
\text { 6) Comparing the } \\
\text { results with the } \\
\text { characteristics given by } \\
\text { the manufacture in the } \\
\text { relay's } \\
\text { manual; 7) Overall, } \\
\text { assigned tasks were } \\
\text { performed competently. }\end{array}$ & $\begin{array}{l}\text { Demonstrated excellent } \\
\text { understanding of the } \\
\text { following tasks: } \\
\text { 1) Identifying the most } \\
\text { important part of an } \\
\text { electromechanical over- } \\
\text { current relay; } \\
\text { 2) Explaining the } \\
\text { application of each part } \\
\text { of the relay; } \\
\text { 3) Determining the pick- } \\
\text { up current of the inverse- } \\
\text { time relay element using } \\
\text { a relay testing } \\
\text { equipment; } 4 \text { ) } \\
\text { Constructing the time- } \\
\text { current curve of the EM } \\
\text { relay; 5) Determining the } \\
\text { instantaneous current of } \\
\text { the inverse-time relay } \\
\text { element using relay } \\
\text { testing equipment; } \\
\text { 6) Comparing the results } \\
\text { with the characteristics } \\
\text { given by the manufacture } \\
\text { in the relay's } \\
\text { manual; } 7 \text { ) Overall, } \\
\text { assigned tasks were } \\
\text { performed competently } \\
\text { and additional } \\
\text { investigations also } \\
\text { conducted. }\end{array}$ & \\
\hline
\end{tabular}




\begin{tabular}{|c|c|c|c|c|}
\hline $\begin{array}{l}\text { TEAM WORK } \\
\text { Outcomes: d }\end{array}$ & $\begin{array}{l}\text { 1) Group does not } \\
\text { function effective as a } \\
\text { team, group dissonance } \\
\text { is apparent.; 2) members } \\
\text { do not contribute } \\
\text { significantly to the team; } \\
\text { 3) group does not } \\
\text { delegate responsibilities } \\
\text { or does so poorly. }\end{array}$ & $\begin{array}{l}\text { 1) Group functions as a } \\
\text { team; 2) members } \\
\text { assume roles as } \\
\text { individual contributors, } \\
\text { moderately enhancing } \\
\text { the team performance; } \\
\text { 3) group effectively } \\
\text { delegates } \\
\text { responsibilities to } \\
\text { manage the project. }\end{array}$ & $\begin{array}{l}\text { 1) Group functions } \\
\text { effectively as a team; } 2 \text { ) } \\
\text { members assume roles as } \\
\text { individual contributors, } \\
\text { significantly enhancing } \\
\text { the team performance; } 3 \text { ) } \\
\text { effectively delegate } \\
\text { responsibilities, } \\
\text { leveraging individual } \\
\text { expertise, interests \& } \\
\text { skills. }\end{array}$ & \\
\hline $\begin{array}{l}\text { Data Analysis } \\
\text { \& } \\
\text { Presentation } \\
\text { Outcomes: } \\
\text { a, b, g }\end{array}$ & $\begin{array}{l}\text { Tables and figures, if } \\
\text { included, 1) are not } \\
\text { numbered, 2) lack } \\
\text { captions, 3) axis not } \\
\text { labeled; 4) data, if } \\
\text { presented, is neither } \\
\text { analyzed nor 5) properly } \\
\text { interpreted; 6) do not } \\
\text { apply engineering } \\
\text { knowledge in the } \\
\text { analysis of data; 7) } \\
\text { report is too long/short; } \\
\text { not well written. }\end{array}$ & $\begin{array}{l}\text { 1) Figures and tables } \\
\text { enhance the report, and } \\
\text { 2) are numbered, 3) } \\
\text { though may lack } \\
\text { descriptive captions, or } \\
\text { 4) have incomplete axis } \\
\text { labels; 5) data presented } \\
\text { is analyzed and 6) } \\
\text { properly interpreted; 7) } \\
\text { apply engineering } \\
\text { knowledge in analysis } \\
\text { of data; 8) report is } \\
\text { concise and well } \\
\text { written. }\end{array}$ & $\begin{array}{l}\text { 1) High quality figures } \\
\text { and tables add depth to } \\
\text { the report, 2) are } \\
\text { numbered, and 3) include } \\
\text { descriptive captions and } \\
\text { 4) well labeled axis; 5) } \\
\text { data presented is clearly } \\
\text { analyzed and 6) expertly } \\
\text { interpreted. 7) clearly } \\
\text { apply engineering } \\
\text { knowledge in analysis of } \\
\text { data; 8) report is concise } \\
\text { and exceptionally well } \\
\text { written. }\end{array}$ & \\
\hline
\end{tabular}

Adopted from rubrics made available by Professor Mary Mueller of Seton Hall University as well as Professor Bob Bass of Portland State University. 


\section{E.6 Coordination of OC Relays in Radial Systems Rubric}

Portland State University Electrical \& Computer Engineering ECE 448/548 Power System Protection I

\section{-Lab 6: Coordination of OC Relays in Radial Systems-}

TA:

GRADE

STUDENT NAMES:

\section{ABET/RES Program Outcomes}

(a) an ability to apply knowledge of mathematics, science, and engineering

(b) an ability to design and conduct experiments, as well as to analyze and interpret data

(d) an ability to function on multi-disciplinary teams

(g) an ability to communicate effectively

\begin{tabular}{|c|c|c|c|c|}
\hline CRITERIA & 1-DEVELOPING & 2-ACCOMPLISHED & 3-EXEMPLARY & SCORE \\
\hline $\begin{array}{l}\text { ASSIGNED } \\
\text { TASKS } \\
\text { Outcomes: b }\end{array}$ & $\begin{array}{l}\text { Did not perform, or did } \\
\text { not show an } \\
\text { understanding of, the } \\
\text { following tasks: } \\
\text { 1) Creating a radial } \\
\text { case in ASPEN } \\
\text { OneLiner containing } \\
\text { the required } \\
\text { information to } \\
\text { do fault studies and } \\
\text { overcurrent relay } \\
\text { coordination; } \\
\text { 2) Introducing data of } \\
\text { phase and ground } \\
\text { overcurrent relay } \\
\text { elements into the } \\
\text { model; } \\
\text { 3) Simulating faults } \\
\text { and determine the } \\
\text { behavior (time) of } \\
\text { overcurrent relay } \\
\text { elements; } \\
\text { 4) Performing a } \\
\text { coordination study to } \\
\text { determine the relay } \\
\text { settings; 5) Overall, } \\
\text { assigned tasks were not } \\
\text { performed } \\
\text { competently. }\end{array}$ & $\begin{array}{l}\text { Performed and } \\
\text { discussed the following } \\
\text { tasks: } \\
\text { 1) Creating a radial case } \\
\text { in ASPEN OneLiner } \\
\text { containing the required } \\
\text { information to } \\
\text { do fault studies and } \\
\text { overcurrent relay } \\
\text { coordination; } \\
\text { 2) Introducing data of } \\
\text { phase and ground } \\
\text { overcurrent relay } \\
\text { elements into the model; } \\
\text { 3) Simulating faults and } \\
\text { determine the behavior } \\
\text { (time) of overcurrent } \\
\text { relay elements; } \\
\text { 4) Performing a } \\
\text { coordination study to } \\
\text { determine the relay } \\
\text { settings; 5) Overall, } \\
\text { assigned tasks were } \\
\text { performed competently. }\end{array}$ & $\begin{array}{l}\text { Demonstrated excellent } \\
\text { understanding of the } \\
\text { following tasks: } \\
\text { 1) Creating a radial case } \\
\text { in ASPEN OneLiner } \\
\text { containing the required } \\
\text { information to } \\
\text { do fault studies and } \\
\text { overcurrent relay } \\
\text { coordination; } \\
\text { 2) Introducing data of } \\
\text { phase and ground } \\
\text { overcurrent relay } \\
\text { elements into the model; } \\
\text { 3) Simulating faults and } \\
\text { determine the behavior } \\
\text { (time) of overcurrent } \\
\text { relay elements; } \\
\text { 4) Performing a } \\
\text { coordination study to } \\
\text { determine the relay } \\
\text { settings; 5) Overall, } \\
\text { assigned tasks were } \\
\text { performed competently } \\
\text { and additional } \\
\text { investigations also } \\
\text { conducted. }\end{array}$ & \\
\hline $\begin{array}{l}\text { TEAM WORK } \\
\text { Outcomes: d }\end{array}$ & $\begin{array}{l}\text { 1) Group does not } \\
\text { function effective as a } \\
\text { team, group dissonance } \\
\text { is apparent.; 2) } \\
\text { members do not } \\
\text { contribute significantly } \\
\text { to the team; } 3 \text { ) group } \\
\text { does not delegate }\end{array}$ & $\begin{array}{l}\text { 1) Group functions as a } \\
\text { team; 2) members } \\
\text { assume roles as } \\
\text { individual contributors, } \\
\text { moderately enhancing } \\
\text { the team performance; } \\
\text { 3) group effectively } \\
\text { delegates }\end{array}$ & $\begin{array}{l}\text { 1) Group functions } \\
\text { effectively as a team; 2) } \\
\text { members assume roles as } \\
\text { individual contributors, } \\
\text { significantly enhancing } \\
\text { the team performance; } 3 \text { ) } \\
\text { effectively delegate } \\
\text { responsibilities, } \\
\text { leveraging individual }\end{array}$ & \\
\hline
\end{tabular}




\begin{tabular}{|c|c|c|c|c|}
\hline & $\begin{array}{l}\text { responsibilities or does } \\
\text { so poorly. }\end{array}$ & $\begin{array}{l}\text { responsibilities to } \\
\text { manage the project. }\end{array}$ & $\begin{array}{l}\text { expertise, interests \& } \\
\text { skills. }\end{array}$ & \\
\hline $\begin{array}{l}\text { Data Analysis } \\
\text { \& } \\
\text { Presentation } \\
\text { Outcomes: } \\
\text { a, b, g }\end{array}$ & $\begin{array}{l}\text { Tables and figures, if } \\
\text { included, 1) are not } \\
\text { numbered, 2) lack } \\
\text { captions, 3) axis not } \\
\text { labeled; 4) data, if } \\
\text { presented, is neither } \\
\text { analyzed nor 5) } \\
\text { properly interpreted; 6) } \\
\text { do not apply } \\
\text { engineering knowledge } \\
\text { in the analysis of data; } \\
\text { 7) report is too } \\
\text { long/short; not well } \\
\text { written. }\end{array}$ & $\begin{array}{l}\text { 1) Figures and tables } \\
\text { enhance the report, and } \\
\text { 2) are numbered, 3) } \\
\text { though may lack } \\
\text { descriptive captions, or } \\
\text { 4) have incomplete axis } \\
\text { labels; 5) data presented } \\
\text { is analyzed and 6) } \\
\text { properly interpreted; 7) } \\
\text { apply engineering } \\
\text { knowledge in analysis } \\
\text { of data; 8) report is } \\
\text { concise and well } \\
\text { written. }\end{array}$ & $\begin{array}{l}\text { 1) High quality figures } \\
\text { and tables add depth to } \\
\text { the report, 2) are } \\
\text { numbered, and 3) include } \\
\text { descriptive captions and } \\
\text { 4) well labeled axis; 5) } \\
\text { data presented is clearly } \\
\text { analyzed and 6) expertly } \\
\text { interpreted. 7) clearly } \\
\text { apply engineering } \\
\text { knowledge in analysis of } \\
\text { data; 8) report is concise } \\
\text { and exceptionally well } \\
\text { written. }\end{array}$ & \\
\hline
\end{tabular}

Adopted from rubrics made available by Professor Mary Mueller of Seton Hall University as well as Professor Bob Bass of Portland State University. 


\section{E.7 Digital Relay Radial Over-current Protection (50/51) Rubric Portland State University Electrical \& Computer Engineering ECE 448/548 Power System Protection I \\ -Lab 7: Digital Relay Radial Over-current Protection (50/51)-}

TA:

GRADE

STUDENT NAMES:
ABET/RES Program Outcomes
(a) an ability to apply knowledge of mathematics, science, and engineering
(b) an ability to design and conduct experiments, as well as to analyze and interpret data
(d) an ability to function on multi-disciplinary teams
(g) an ability to communicate effectively

\begin{tabular}{|c|c|c|c|c|}
\hline CRITERIA & 1-DEVELOPING & 2-ACCOMPLISHED & 3-EXEMPLARY & SCORE \\
\hline $\begin{array}{l}\text { ASSIGNED } \\
\text { TASKS } \\
\text { Outcomes: b }\end{array}$ & $\begin{array}{l}\text { Did not perform, or did } \\
\text { not show an } \\
\text { understanding of, the } \\
\text { following tasks: } \\
\text { 1)Implement physical } \\
\text { set-up to test SEL-351 } \\
\text { using the SEL-AMS; } \\
\text { 2) Introduce settings to } \\
\text { the relay (phase and } \\
\text { ground elements); } \\
\text { 3)Properly set a test usi } \\
\text { ng the SEL-5410 softwa } \\
\text { re; 4) Communicate } \\
\text { with the SEL-551 using } \\
\text { the SEL-AMS;5) Test } \\
\text { the relay with the SEL- } \\
\text { AMS; 6) Verify } \\
\text { operation results by } \\
\text { comparing with } \\
\text { simulated results found } \\
\text { using ASPEN software; } \\
\text { 7) Overall, assigned } \\
\text { tasks were not } \\
\text { performed competently. }\end{array}$ & $\begin{array}{l}\text { Performed and } \\
\text { discussed the following } \\
\text { tasks: } \\
\text { 1)Implement physical } \\
\text { set-up to test SEL-551 } \\
\text { using SEL-AMS; } \\
\text { 2) Introduce settings to } \\
\text { the relay (phase and } \\
\text { ground elements); } \\
\text { 3) Properly set a test } \\
\text { using the SEL-5410 } \\
\text { software; } \\
\text { 4) Communicate with } \\
\text { the SEL-551 using the } \\
\text { SEL-AMS; 5)Test the } \\
\text { relay with the SEL- } \\
\text { AMS; 6) Verify } \\
\text { operation results by } \\
\text { comparing with } \\
\text { simulated results found } \\
\text { using ASPEN software; } \\
\text { 7) Overall, assigned } \\
\text { tasks were performed } \\
\text { competently. }\end{array}$ & $\begin{array}{l}\text { Demonstrated excellent } \\
\text { understanding of the } \\
\text { following tasks: } \\
\text { 1) Implement physical } \\
\text { set-up to test SEL-551 } \\
\text { using SEL-AMS; } \\
\text { 2) Introduce settings to } \\
\text { the relay (phase and } \\
\text { ground elements); } \\
\text { 3) Properly set a test } \\
\text { using the SEL-5410 } \\
\text { software; } \\
\text { 4) Communicate with } \\
\text { the SEL-551 using the } \\
\text { SEL-AMS; 5)Test the } \\
\text { relay with the SEL- } \\
\text { AMS; 6) Verify } \\
\text { operation results by } \\
\text { comparing with } \\
\text { simulated results found } \\
\text { using ASPEN software; } \\
\text { 7) Overall, assigned } \\
\text { tasks were performed } \\
\text { competently and } \\
\text { additional } \\
\text { investigations also were } \\
\text { conducted. }\end{array}$ & \\
\hline $\begin{array}{l}\text { TEAM WORK } \\
\text { Outcomes: d }\end{array}$ & $\begin{array}{l}\text { 1) Group does not } \\
\text { function effective as a } \\
\text { team, group dissonance } \\
\text { is apparent.; 2) } \\
\text { members do not } \\
\text { contribute significantly } \\
\text { to the team; 3) group } \\
\text { does not delegate } \\
\text { responsibilities or does } \\
\text { so poorly. }\end{array}$ & $\begin{array}{l}\text { 1) Group functions as a } \\
\text { team; 2) members } \\
\text { assume roles as } \\
\text { individual contributors, } \\
\text { moderately enhancing } \\
\text { the team performance; } \\
\text { 3) group effectively } \\
\text { delegates } \\
\text { responsibilities to } \\
\text { manage the project. }\end{array}$ & $\begin{array}{l}\text { 1) Group functions } \\
\text { effectively as a team; 2) } \\
\text { members assume roles } \\
\text { as individual } \\
\text { contributors, } \\
\text { significantly enhancing } \\
\text { the team performance; } \\
\text { 3) effectively delegate } \\
\text { responsibilities, } \\
\text { leveraging individual }\end{array}$ & \\
\hline
\end{tabular}




\begin{tabular}{|c|c|c|c|c|}
\hline & & & $\begin{array}{l}\text { expertise, interests \& } \\
\text { skills. }\end{array}$ & \\
\hline $\begin{array}{l}\text { DAta Analysis } \\
\text { \& } \\
\text { Presentation } \\
\text { Outcomes: } \\
\text { a, b, g }\end{array}$ & $\begin{array}{l}\text { Tables and figures, if } \\
\text { included, 1) are not } \\
\text { numbered, 2) lack } \\
\text { captions, 3) axis not } \\
\text { labeled; 4) data, if } \\
\text { presented, is neither } \\
\text { analyzed nor 5) } \\
\text { properly interpreted; 6) } \\
\text { do not apply } \\
\text { engineering knowledge } \\
\text { in the analysis of data; } \\
\text { 7) report is too } \\
\text { long/short; not well } \\
\text { written. }\end{array}$ & $\begin{array}{l}\text { 1) Figures and tables } \\
\text { enhance the report, and } \\
\text { 2) are numbered, 3) } \\
\text { though may lack } \\
\text { descriptive captions, or } \\
\text { 4) have incomplete axis } \\
\text { labels; 5) data presented } \\
\text { is analyzed and 6) } \\
\text { properly interpreted; 7) } \\
\text { apply engineering } \\
\text { knowledge in analysis } \\
\text { of data; 8) report is } \\
\text { concise and well } \\
\text { written. }\end{array}$ & $\begin{array}{l}\text { 1) High quality figures } \\
\text { and tables add depth to } \\
\text { the report, 2) are } \\
\text { numbered, and 3) } \\
\text { include descriptive } \\
\text { captions and 4) well } \\
\text { labeled axis; 5) data } \\
\text { presented is clearly } \\
\text { analyzed and 6) } \\
\text { expertly interpreted. 7) } \\
\text { clearly apply } \\
\text { engineering knowledge } \\
\text { in analysis of data; 8) } \\
\text { report is concise and } \\
\text { exceptionally well } \\
\text { written. }\end{array}$ & \\
\hline
\end{tabular}

Adopted from rubrics made available by Professor Mary Mueller of Seton Hall University as well as Professor Bob Bass of Portland State University. 


\title{
E.8 ASPEN Looped System Coordination Rubric
}

\author{
Portland State University \\ Electrical \& Computer Engineering \\ ECE 448/548 Power System Protection I \\ -Lab 8: ASPEN Looped System Coordination-
}

TA:

STUDENT NAMES:

GRADE

\section{ABET/RES Program Outcomes}

(a) an ability to apply knowledge of mathematics, science, and engineering

(b) an ability to design and conduct experiments, as well as to analyze and interpret data

(d) an ability to function on multi-disciplinary teams

(g) an ability to communicate effectively

\begin{tabular}{|c|c|c|c|c|}
\hline CRITERIA & 1-DEVELOPING & 2-ACCOMPLISHED & 3-EXEMPLARY & SCORE \\
\hline $\begin{array}{l}\text { ASSIGNED } \\
\text { TASKS } \\
\text { Outcomes: b }\end{array}$ & $\begin{array}{l}\text { Did not perform, or did } \\
\text { not show an } \\
\text { understanding of, the } \\
\text { following tasks: } \\
\text { 1)Create a looped case in } \\
\text { ASPEN OneLiner } \\
\text { containing the required } \\
\text { information to } \\
\text { perform fault studies and } \\
\text { overcurrent relay } \\
\text { coordination; } \\
\text { 2)Introduce data of phase } \\
\text { and ground directional } \\
\text { overcurrent relay } \\
\text { elements into } \\
\text { the model; } \\
\text { 3)Simulate faults and } \\
\text { determine the behavior } \\
\text { (time) of overcurrent } \\
\text { relay elements; } \\
\text { 4)Perform a coordination } \\
\text { study to determine the } \\
\text { relay settings; }\end{array}$ & $\begin{array}{l}\text { Performed and discussed } \\
\text { the following tasks: } \\
\text { 1)Create a looped case } \\
\text { in ASPEN OneLiner } \\
\text { containing the required } \\
\text { information to } \\
\text { perform fault studies } \\
\text { and overcurrent relay } \\
\text { coordination; } \\
\text { 2)Introduce data of } \\
\text { phase and ground } \\
\text { directional overcurrent } \\
\text { relay elements into } \\
\text { the model; } \\
\text { 3)Simulate faults and } \\
\text { determine the behavior } \\
\text { (time) of overcurrent } \\
\text { relay elements; } \\
\text { 4)Perform a } \\
\text { coordination study to } \\
\text { determine the relay } \\
\text { settings; }\end{array}$ & $\begin{array}{l}\text { Demonstrated excellent } \\
\text { understanding of the } \\
\text { following tasks: } \\
\text { 1)Create a looped case in } \\
\text { ASPEN OneLiner } \\
\text { containing the required } \\
\text { information to } \\
\text { perform fault studies and } \\
\text { overcurrent relay } \\
\text { coordination; } \\
\text { 2)Introduce data of } \\
\text { phase and ground } \\
\text { directional overcurrent } \\
\text { relay elements into } \\
\text { the model; } \\
\text { 3)Simulate faults and } \\
\text { determine the behavior } \\
\text { (time) of overcurrent } \\
\text { relay elements; } \\
\text { 4)Perform a coordination } \\
\text { study to determine the } \\
\text { relay settings; }\end{array}$ & \\
\hline $\begin{array}{l}\text { TEAM WORK } \\
\text { Outcomes: d }\end{array}$ & $\begin{array}{l}\text { 1) Group does not } \\
\text { function effective as a } \\
\text { team, group dissonance is } \\
\text { apparent.; 2) members do } \\
\text { not contribute } \\
\text { significantly to the team; } \\
\text { 3) group does not } \\
\text { delegate responsibilities } \\
\text { or does so poorly. }\end{array}$ & $\begin{array}{l}\text { 1) Group functions as a } \\
\text { team; 2) members } \\
\text { assume roles as } \\
\text { individual contributors, } \\
\text { moderately enhancing } \\
\text { the team performance; } \\
\text { 3) group effectively } \\
\text { delegates } \\
\text { responsibilities to } \\
\text { manage the project. }\end{array}$ & $\begin{array}{l}\text { 1) Group functions } \\
\text { effectively as a team; 2) } \\
\text { members assume roles as } \\
\text { individual contributors, } \\
\text { significantly enhancing } \\
\text { the team performance; } 3 \text { ) } \\
\text { effectively delegate } \\
\text { responsibilities, } \\
\text { leveraging individual } \\
\text { expertise, interests \& } \\
\text { skills. }\end{array}$ & \\
\hline
\end{tabular}




\begin{tabular}{|c|c|c|c|c|}
\hline $\begin{array}{l}\text { Data Analysis } \\
\text { \& } \\
\text { Presentation } \\
\text { Outcomes: } \\
\text { a, b, g }\end{array}$ & $\begin{array}{l}\text { Tables and figures, if } \\
\text { included, 1) are not } \\
\text { numbered, 2) lack } \\
\text { captions, 3) axis not } \\
\text { labeled; 4) data, if } \\
\text { presented, is neither } \\
\text { analyzed nor 5) } \\
\text { properly interpreted; 6) } \\
\text { do not apply } \\
\text { engineering knowledge } \\
\text { in the analysis of data; } \\
\text { 7) report is too } \\
\text { long/short; not well } \\
\text { written. }\end{array}$ & $\begin{array}{l}\text { 1) Figures and tables } \\
\text { enhance the report, and } \\
\text { 2) are numbered, 3) } \\
\text { though may lack } \\
\text { descriptive captions, or } \\
\text { 4) have incomplete axis } \\
\text { labels; 5) data presented } \\
\text { is analyzed and 6) } \\
\text { properly interpreted; 7) } \\
\text { apply engineering } \\
\text { knowledge in analysis of } \\
\text { data; 8) report is concise } \\
\text { and well written. }\end{array}$ & $\begin{array}{l}\text { 1) High quality figures } \\
\text { and tables add depth to } \\
\text { the report, 2) are } \\
\text { numbered, and 3) } \\
\text { include descriptive } \\
\text { captions and 4) well } \\
\text { labeled axis; 5) data } \\
\text { presented is clearly } \\
\text { analyzed and 6) expertly } \\
\text { interpreted; 7) clearly } \\
\text { apply engineering } \\
\text { knowledge in analysis of } \\
\text { data; 8) report is concise } \\
\text { and exceptionally well } \\
\text { written. }\end{array}$ & $/ 5$ \\
\hline
\end{tabular}

Adopted from rubrics made available by Professor Mary Mueller of Seton Hall University as well as Professor Bob Bass of Portland State University. 


\title{
E.9 Directional Over-current Relays (67) Rubric
}

\author{
Portland State University \\ Electrical \& Computer Engineering \\ ECE 448/548 Power System Protection I \\ -Lab 9: Directional Over-current Relays (67) -
}

TA:

GRADE

STUDENT NAMES:

\begin{tabular}{|c|c|c|c|c|}
\hline \multicolumn{5}{|c|}{$\begin{array}{l}\text { ABET/RES Program Outcomes } \\
\text { (a) an ability to apply knowledge of mathematics, science, and engineering } \\
\text { (b) an ability to design and conduct experiments, as well as to analyze and interpret data } \\
\text { (d) an ability to function on multi-disciplinary teams } \\
\text { (g) an ability to communicate effectively }\end{array}$} \\
\hline CRITERIA & 1-DEVELOPING & 2-ACCOMPLISHED & 3-EXEMPLARY & SCORE \\
\hline $\begin{array}{l}\text { ASSIGNED } \\
\text { TASKS } \\
\text { Outcomes: b }\end{array}$ & $\begin{array}{l}\text { Did not perform, or } \\
\text { did not show an } \\
\text { understanding of, the } \\
\text { following tasks: } \\
\text { 1)Identify the } \\
\text { different parts of an } \\
\text { electromechanical } \\
\text { directional over- } \\
\text { current relay 2) } \\
\text { Determine the } \\
\text { operation zone of an } \\
\text { electromechanical } \\
\text { directional element } \\
\text { using relay testing } \\
\text { equipment; } \\
\text { 3) Implement physical } \\
\text { set-up to test SEL-351 } \\
\text { using the SEL-RTS } \\
\text { (AMS); } \\
\text { 4) Introduce settings } \\
\text { to the relay (Phase and } \\
\text { Neutral Ground } \\
\text { elements); 5) Test the } \\
\text { SEL-351 relay with } \\
\text { SEL-5401 software } \\
\text { and obtain operation } \\
\text { times; 6) Compare } \\
\text { results to those found } \\
\text { by theoretical } \\
\text { calculation. }\end{array}$ & $\begin{array}{l}\text { Performed and } \\
\text { discussed the following } \\
\text { tasks: } \\
\text { 1)Identify the different } \\
\text { parts of an } \\
\text { electromechanical } \\
\text { directional over-current } \\
\text { relay 2) Determine the } \\
\text { operation zone of an } \\
\text { electromechanical } \\
\text { directional element } \\
\text { using relay testing } \\
\text { equipment; } \\
\text { 3) Implement physical } \\
\text { set-up to test SEL-351 } \\
\text { using the SEL-RTS } \\
\text { (AMS); } \\
\text { 4) Introduce settings to } \\
\text { the relay (Phase and } \\
\text { Neutral Ground } \\
\text { elements); 5) Test the } \\
\text { SEL-351 relay with } \\
\text { SEL-5401 software and } \\
\text { obtain operation times; } \\
\text { 6) Compare results to } \\
\text { those found by } \\
\text { theoretical calculation. }\end{array}$ & $\begin{array}{l}\text { Demonstrated excellent } \\
\text { understanding of the } \\
\text { following tasks: } \\
\text { 1)Identify the different } \\
\text { parts of an } \\
\text { electromechanical } \\
\text { directional over-current } \\
\text { relay 2) Determine the } \\
\text { operation zone of an } \\
\text { electromechanical } \\
\text { directional element } \\
\text { using relay testing } \\
\text { equipment; } \\
\text { 3) Implement physical } \\
\text { set-up to test SEL-351 } \\
\text { using the SEL-RTS } \\
\text { (AMS); } \\
\text { 4) Introduce settings to } \\
\text { the relay (Phase and } \\
\text { Neutral Ground } \\
\text { elements); 5) Test the } \\
\text { SEL-351 relay with } \\
\text { SEL-5401 software and } \\
\text { obtain operation times; } \\
\text { 6) Compare results to } \\
\text { those found by } \\
\text { theoretical calculation. }\end{array}$ & \\
\hline
\end{tabular}




\begin{tabular}{|c|c|c|c|c|}
\hline $\begin{array}{l}\text { TEAM WORK } \\
\text { Outcomes: d }\end{array}$ & $\begin{array}{l}\text { 1) Group does not } \\
\text { function effective as a } \\
\text { team, group } \\
\text { dissonance is } \\
\text { apparent.; 2) members } \\
\text { do not contribute } \\
\text { significantly to the } \\
\text { team; } 3 \text { ) group does } \\
\text { not delegate } \\
\text { responsibilities or } \\
\text { does so poorly. }\end{array}$ & $\begin{array}{l}\text { 1) Group functions as a } \\
\text { team; 2) members } \\
\text { assume roles as } \\
\text { individual contributors, } \\
\text { moderately enhancing } \\
\text { the team performance; } \\
\text { 3) group effectively } \\
\text { delegates } \\
\text { responsibilities to } \\
\text { manage the project. }\end{array}$ & $\begin{array}{l}\text { 1) Group functions } \\
\text { effectively as a team; 2) } \\
\text { members assume roles } \\
\text { as individual } \\
\text { contributors, } \\
\text { significantly enhancing } \\
\text { the team performance; } \\
\text { 3) effectively delegate } \\
\text { responsibilities, } \\
\text { leveraging individual } \\
\text { expertise, interests \& } \\
\text { skills. }\end{array}$ & 5 \\
\hline $\begin{array}{l}\text { DAta ANALYSiS } \\
\text { \& } \\
\text { PreSENTAtion } \\
\text { Outcomes: } \\
\text { a, b, g }\end{array}$ & $\begin{array}{l}\text { Tables and figures, if } \\
\text { included, 1) are not } \\
\text { numbered, 2) lack } \\
\text { captions, 3) axis not } \\
\text { labeled; 4) data, if } \\
\text { presented, is neither } \\
\text { analyzed nor 5) } \\
\text { properly interpreted; } \\
\text { 6) do not apply } \\
\text { engineering } \\
\text { knowledge in the } \\
\text { analysis of data; 7) } \\
\text { report is too } \\
\text { long/short; not well } \\
\text { written. }\end{array}$ & $\begin{array}{l}\text { 1) Figures and tables } \\
\text { enhance the report, and } \\
\text { 2) are numbered, 3) } \\
\text { though may lack } \\
\text { descriptive captions, or } \\
\text { 4) have incomplete axis } \\
\text { labels; 5) data } \\
\text { presented is analyzed } \\
\text { and 6) properly } \\
\text { interpreted; 7) apply } \\
\text { engineering knowledge } \\
\text { in analysis of data; 8) } \\
\text { report is concise and } \\
\text { well written. }\end{array}$ & $\begin{array}{l}\text { 1) High quality figures } \\
\text { and tables add depth to } \\
\text { the report, 2) are } \\
\text { numbered, and 3) } \\
\text { include descriptive } \\
\text { captions and 4) well } \\
\text { labeled axis; 5) data } \\
\text { presented is clearly } \\
\text { analyzed and 6) expertly } \\
\text { interpreted; 7) clearly } \\
\text { apply engineering } \\
\text { knowledge in analysis } \\
\text { of data; 8) report is } \\
\text { concise and } \\
\text { exceptionally well } \\
\text { written. }\end{array}$ & \\
\hline
\end{tabular}

Adopted from rubrics made available by Professor Mary Mueller of Seton Hall University as well as Professor Bob Bass of Portland State University. 


\title{
Appendix F: Laboratory Course Syllabus
}

\author{
Portland State University \\ Electrical \& Computer Engineering
}

-ECE 410/548L Power Systems Protection Lab-

Hours: $\quad 3$ hours per week

Instructors: Jennifer Ferris

Shailabh Mazari

Kalyani Abhyankar

Robert Bass, Ph.D. (supervisor)

Email: jennifer.b.ferris@gmail.com

Email: smazari@pdx.edu

Email: kalyani@pdx.edu

Email: robert.bass@pdx.edu

Description: An educational lab focusing on basic elements of power system protection theory and application using industry standard software and equipment. This lab is designed to directly follow lecture course material as a direct application of concepts.

References: Protective Relaying: Principles and Applications (Third Edition), by J. L. Blackburn \& T. J. Domin, Marcel Dekker Inc., 2007.

http://www.osinerg.gob.pe/newweb/uploads/GFE/eventos/EVENTO\%207/TEXT O\%207A.pdf

\section{Required}

Lab Supplies: TBD

Instruction: The class will meet once per week for three hours. Laboratory activities include three laboratory teaching focused on different aspects of electrical power systems.

Meeting

ECE 410/548L L01: Tuesday, 6:40pm-9:30pm

Times:

ECE 410/548L L02: Thursday, 6:40pm-9:30pm

ECE 410/548L L03: Monday, 1:30pm-4:20pm

ECE 410/548L L04: Wednesday, 1:30pm-4:20pm

ECE 410/548L L05: Tuesday, 1:30pm-4:20pm

ECE 410/548L L06: Thursday, 1:30pm-4:20pm 


\section{Course Objectives:}

Upon completion of ECE 410/548 Lab, students should be able to:

- Follow safe electrical work practices

- Use software to simulate and study radial and looped power systems

- Verify through experimentation the concepts of thermal damage, fuse element protection curves, relay current pick-up, and relay operation time.

- Design simple power system protection coordination, simulate power flows and faults, and verify design effectiveness.

\section{Lab Assignments:}

1. Electrical Safety Quiz

a. Students must pass this quiz with an $80 \%$ or greater before working with the 120 $\mathrm{V}_{\mathrm{AC}}$ relay testing equipment beyond Week 1.

2. Labs

a. Nine labs featuring various power systems protection topics

b. Students investigate and characterize various power systems protection elements

3. Written Presentation of Lab Assignments

a. Written reports of lab experiments are due EOB on Friday of the following week.

Lab Schedule:

\begin{tabular}{|l|l|l|}
\hline Week 1 & Tasks & Action Items \\
\hline Week 2 & Wire Heating and Fuses & $\begin{array}{l}\text { Introduction to ASPEN software using radial and } \\
\text { looped system models. }\end{array}$ \\
\hline Week 3 & Auxiliary Relay Testing & $\begin{array}{l}\text { Verify theoretical outcomes to thermal curves of } \\
\text { both temperature and current through } \\
\text { experimentation. }\end{array}$ \\
\hline Week 4 & EM Relay Radial Over-current Protection & Introduction to EM equipment and relay testing. \\
\hline Week 5 & Coordination of OC Relays in Radial Systems & $\begin{array}{l}\text { Introduction to the coordination of over-current } \\
\text { elements in a radial power system circuit. }\end{array}$ \\
\hline Week 6 & Digital Relay Radial Over-current Protection & $\begin{array}{l}\text { Introduction to the coordination of over-current } \\
\text { relays in a radial power system using an industry } \\
\text { software program. }\end{array}$ \\
\hline Week 7 & Directional Over-current Relays & $\begin{array}{l}\text { Testing of directional over-current elements on } \\
\text { EM and digital relays. }\end{array}$ \\
\hline Week 8 & Protection Coordination of Looped Systems & $\begin{array}{l}\text { Introduction to the coordination of over-current } \\
\text { relays in a looped power system using an industry } \\
\text { software program. }\end{array}$ \\
\hline Week 9 & Distance Relay, SEL-2411, SEL-3505 & $\begin{array}{l}\text { Program and test distance relay settings or choose } \\
\text { one of the listed alternative relays to program and } \\
\text { test. }\end{array}$ \\
\hline
\end{tabular}

Any student with a disability who anticipates a need for accommodation in this course is encouraged to talk to the instructor about their needs as soon as possible. 
Appendix G: Phase III Bill of Materials (BOMs) 
G.1 BOM 1 - General panel materials list for drawings C.1 - C.14

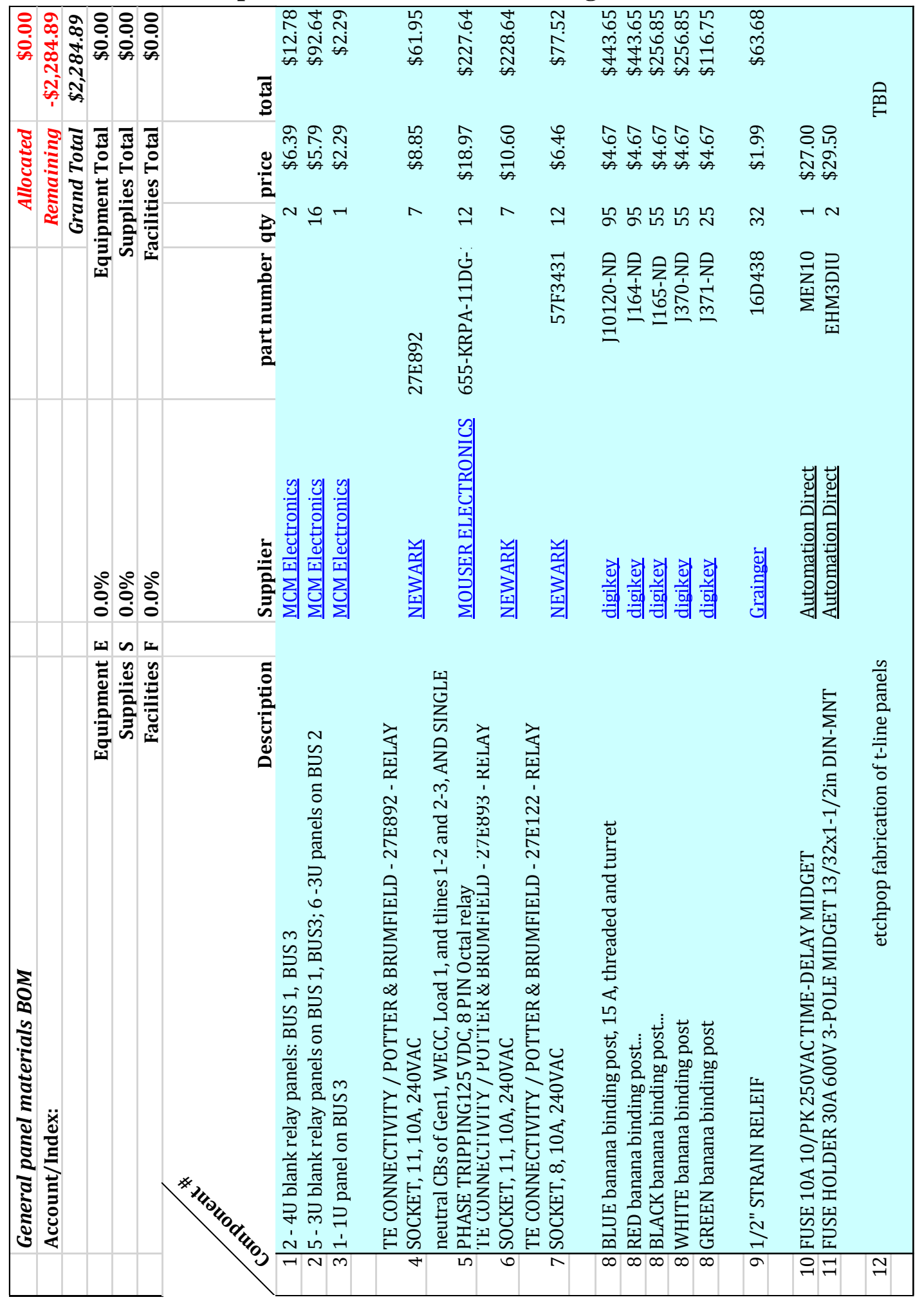




\section{G.2 Generator BOM}

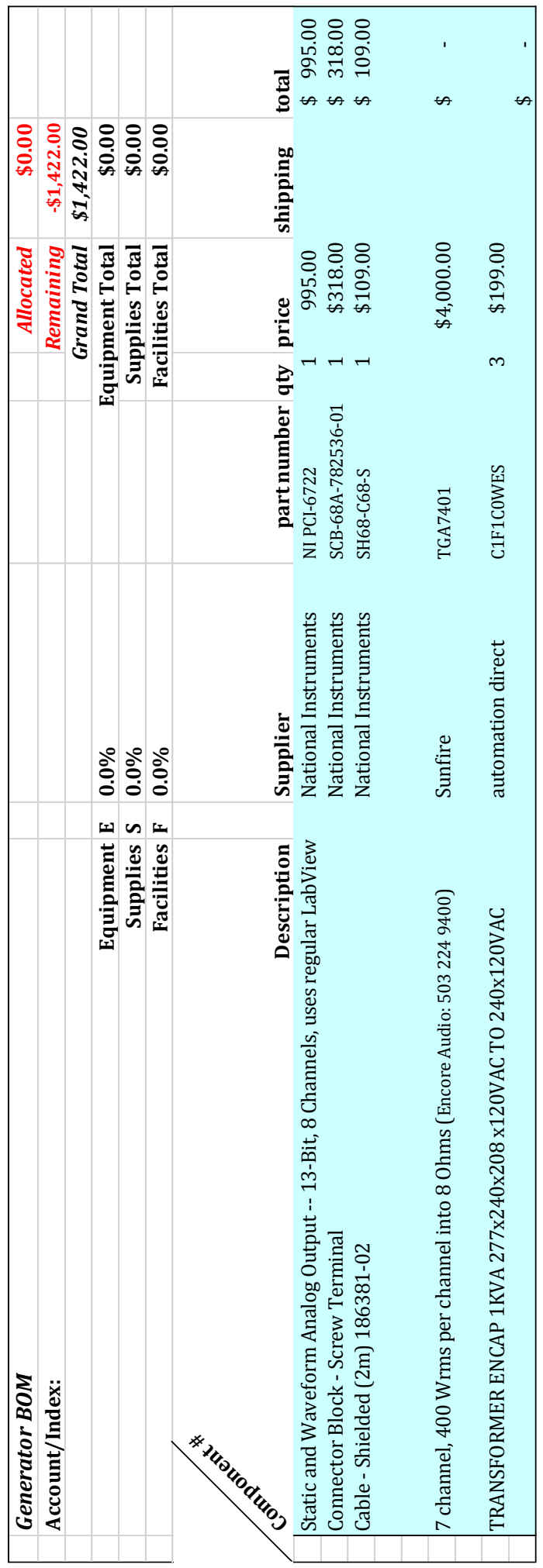




\section{G.3 Transmission line module BOM}

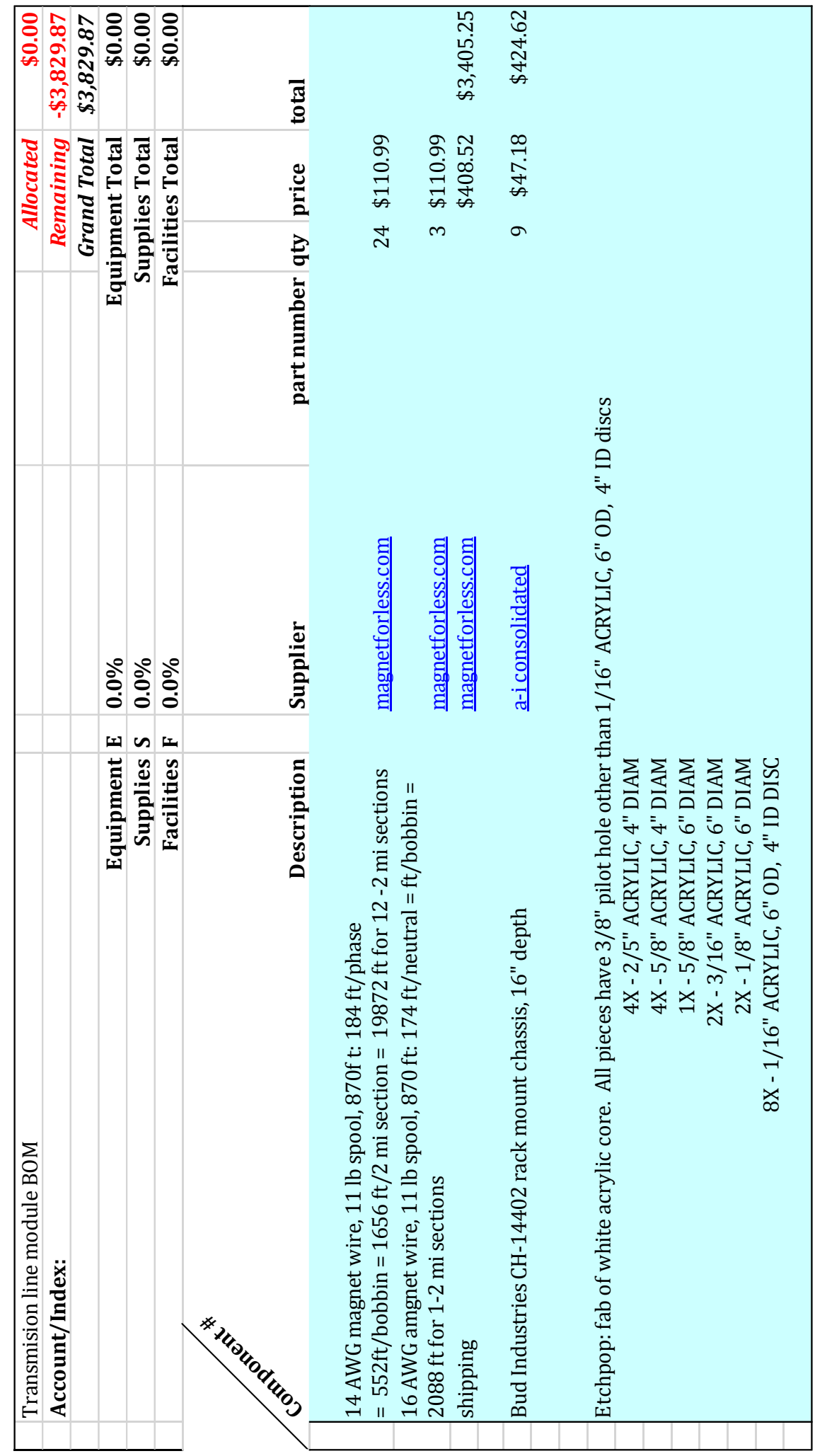


G.4 Sync check panel BOM

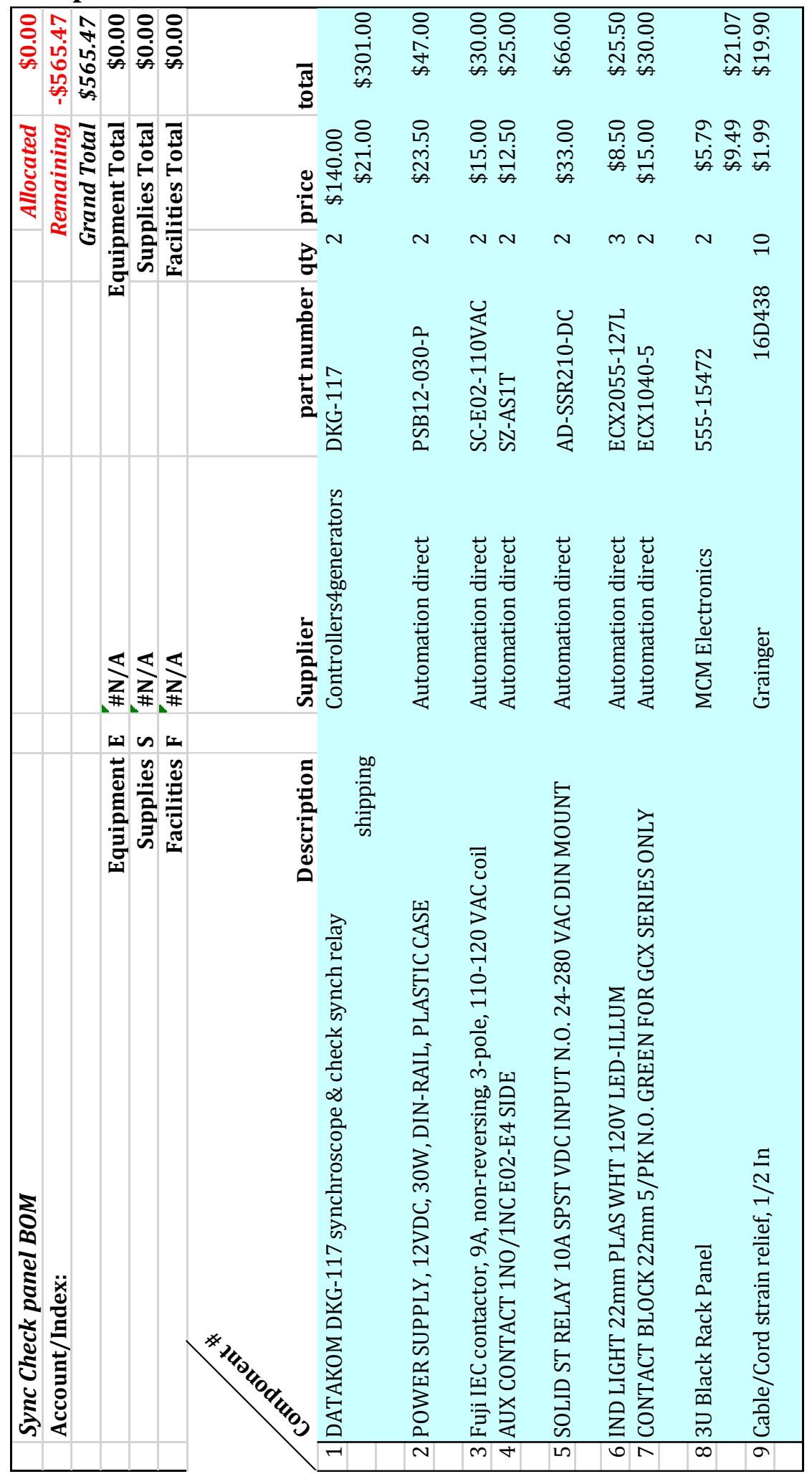




\section{G.5 Electromechanical relays and relay testers BOM}

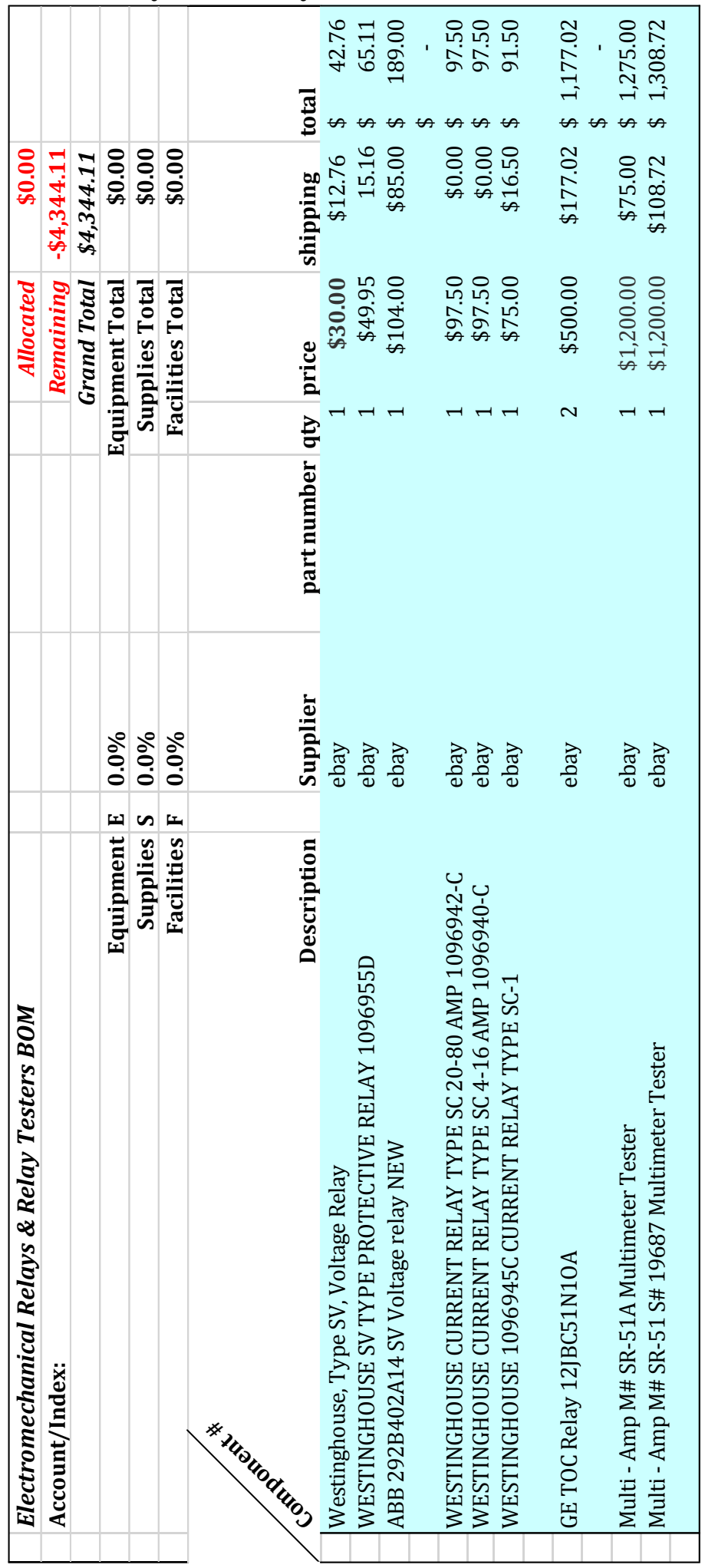




\section{G.6 Fuse holder materials BOM}

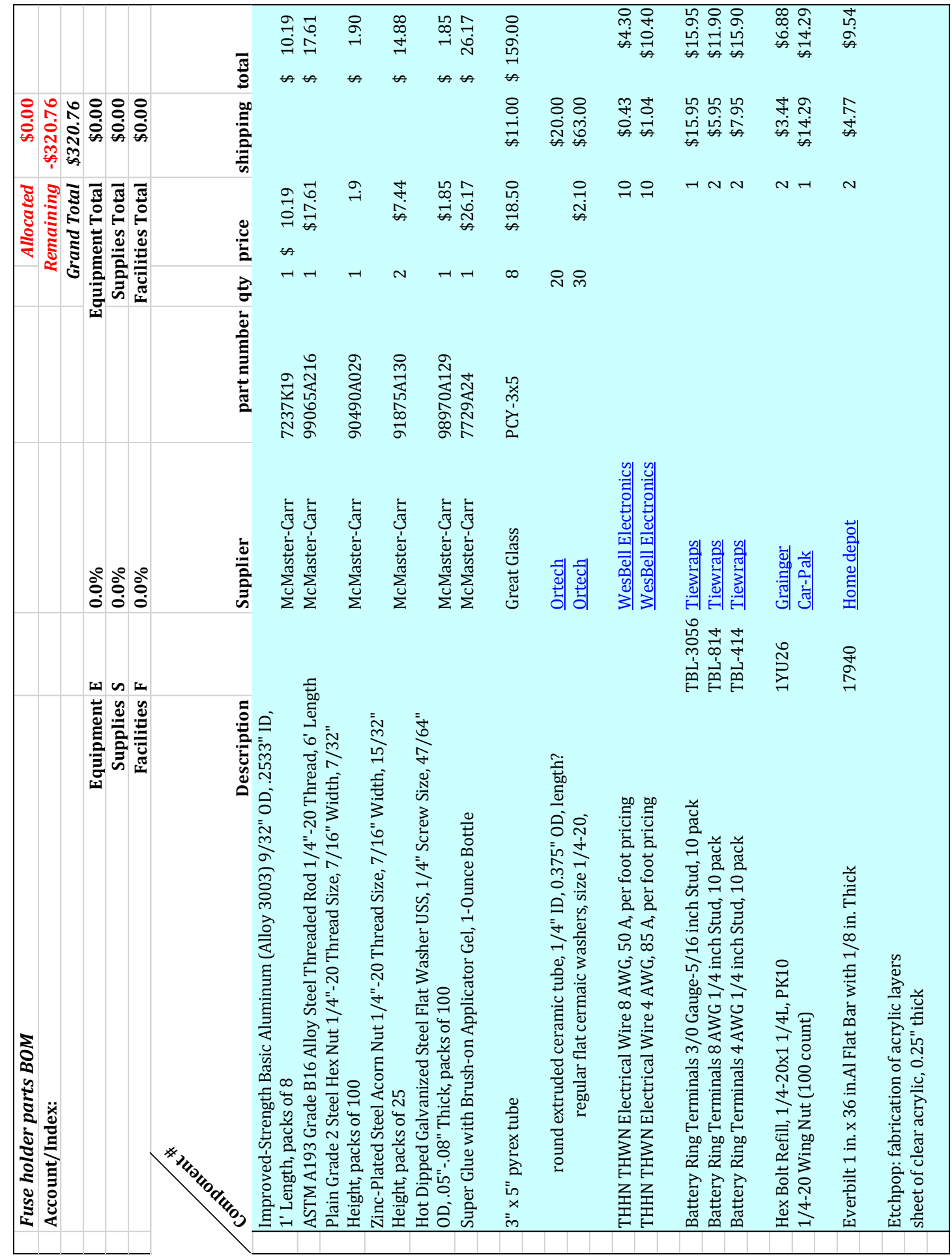




\section{G.7 Current Transformers and rheostat BOM}

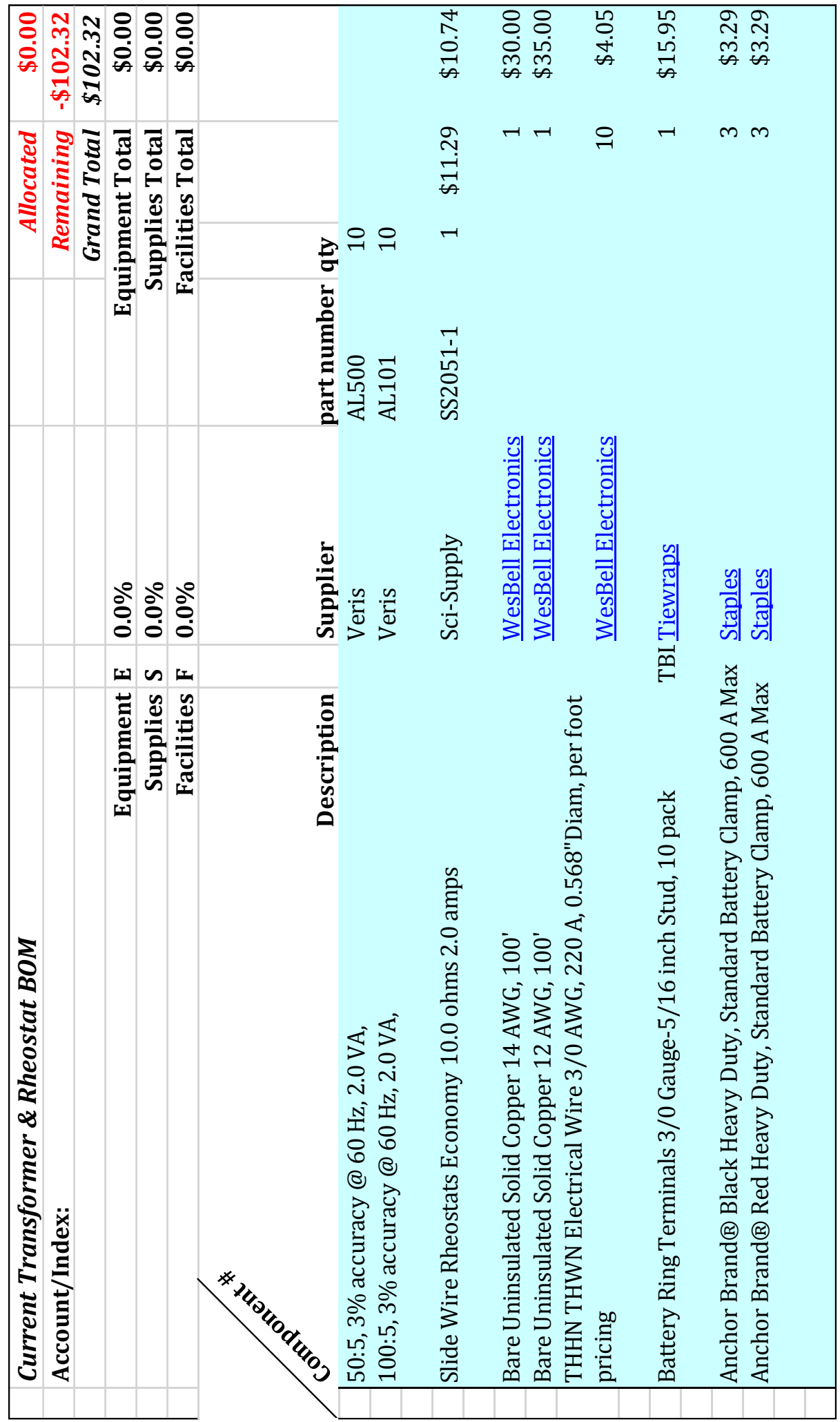




\section{Appendix F: Student Survey Comments}

\section{Lab 1: ASPEN Introduction}

- A link to or adding in a quick review of calculating faults would be a handy addition.

- Aspen is like powerworld but alot easier to navigate. The symbols are clear.

- Aspen seemed like a "clunky" application because it required all that data entry for every piece of the network. I guess in that respect it was no better than PowerWorld or other similar packages. It seemed to me like I put a lot of effort into making Aspen work for not a lot of gain. Maybe I need to spend more "quality" time with it. I liked the specific instructions of what to do ... maybe having a tutorial as a reference on D2L -- I looked for one myself and found a document that helped me limp along.

- Great introduction to ASPEN!

- Had to figure some things out in ASPEN but was good learning experience as an engineer.

- I wish the diagrams had all of the required information before starting the lab. I also wish there was an explanation of how to read the TTY results page.

- It would be nice to have ASPEN installed in other labs for practicing and further studying further

- Might be better to start with a simpler model.

- More explanation on how to use need to be put in the lab

- N/A. However, it would be nice if there were a way to batch calculate faults.

- providing more information on how to read the tty window will helpful

- quick run through on the new software would be nice. I know there is not alot of time to do this is, but it would be helpful 
- The fact that the lab is divided into multiple groups is a really plus to the lab, really great TA, and a good learning experience all in all.

- The handout to perform the lab was missing quite a bit of information. We had to make quite a few assumptions about impedences, voltages, etc., especially on section 2. Also, there could have been a little more information or explanation in the handout on how to read the TTY table. The handout was not clear on which values were needed for the write up.

- Took a while to get the hand of Aspen's interface. After some effort, the TTL output could be easily interpreted.

- $\quad$ very good experience

- Very good introduction to ASPEN

- Yes, it meets all the requirement.

Lab 2: Thermal properties - wire heating and fuses

- Fun lab

- Lab was well explained.

- $\quad$ please wrap-text $\mathrm{Q} \# 4$ for easier reading. tnx

- thanks!

- This lab was fun as well as informative.

- This lab was interesting in the fact that the damage temps/curves were physically measured. The frustrating part was that the SR51 supply unit did not work correctly which wasted our time. We did not get the correct time to current measurements due to this fact.

- This lab work was helpful. 
- this was lab was really great as we had a hands-on experience on wire-heating, and melting time.

- we enjoyed it.

- Went pretty well.

Lab 3: Auxiliary relay testing

- Good, simple little lab. This was a good way to get our hands dirty with actual devices. Thanks.

- great experiance

- Great experience using a real life machine. The TAs were awesome

- $\quad$ i guess i learned more than i expected

- I know it would be much, but do you think next year senior project you could have some seniors build a better protection system that is more up to speed with what is happening today. I.E. software interface, dials and buttons that work.

- I liked it!

- It is really really good. He is really really helpful.

- It was nice to see the components in person.

- It would be a little more clear which user manual and settings were for which relay. The part numbers on the SV and SC relays did not match the user manuals so I had to do some research. This could have been a little more clear.

- It's a great lab assignment overall. Learned more on relay (voltage/current)

- Lab assignment: great, have the student get their hands on this equipment which is truly ubiquitous in the industry. Awesome experience! Procedures: thank you for making whatever manuals available as a resource on D2L. Some of these things were made before many of us were born, so said manuals can be hard to find (not impossible) with a 
mere Google search. Having that resource available saved valuable time that the student can spend interacting with the relays. TA instruction: outstanding! It's very difficult to learn something new when you don't even know what you're looking at. Framing what the relay does, what it's components are, how it might function, was a good start for the student down the path of thinking about how this device might be used in practice in the field. I assure you that even if the TA would "spoon feed" information about every detail of the relay, there would still be questions. And that's where the learning is, in students' asking questions. Also, the test equipment was not necessarily intuitive, so having a bit of help with it saved valuable time that the student can use thinking about the relay and how it works, not being frustrated about how the damned testing machine works.

Overall, this lab gives students a good launching point for their own discovery and independent thought about relays in general and these long-in-the-tooth, but reliable mastodons. Can we have more play time, please?!

- Maybe little more info on what is each knob is there for or it do.

- Thanks for the experiment!

- that was my first experience with relays. If that was the most basic, then that was great

- The TAs are really helpful.

- this lab was fun and I learned something new

- a more in-depth explanation of lab

- This one is gonna take some practice. I wish there were a way that we could have been led to "discover" more.

- Very helpful

- Very interesting. Would be better if there were more relay types to test. 


\section{Lab 4: Current transformer testing}

- The correct or expected CT curves did not really occur on our 2 units. Since the CT manufacturer does not have any curves on their website data sheet, it was difficult to analyze and figure out what went wrong.

- the lab was really helpful. maybe it would be more helpful to have the software that downloads the screenshots from the oscilloscope to the station computer.

- we learned about relays more.

- CT used was highly magnetized during all experiments, hence the magnetization curve obtained was not a good reflection of its accuracy and range. Having additional CT's to use for a "sacrificial" experiment and then collect useful data with would be an improvement.

- ironic using a more accurate CT to measure the intended CT

- It was great to have some hands-on with CTs.

- This lab was trouble from the beginning. Mainly due to the condition of the equipment.

- the data sheet did not have any kind of curves so there was nothing to compare to

- We had some problem in the results and we shifted the graphs according to that.

- The CT we used didn't magnetize. So we had to estimate our current and voltage ratio

- CT's a bit difficult to calibrate (keeps staying at saturation)

- There were so many issues with the CT's that it was hard to concentrate on the objectives of the lab. Hopefully, some more experimentation (and preparation) time will be helpful to making it a better lab.

\section{Lab 5: EM Relay Radial Over-current Protection}

- determining the instantaneous current was a bit difficult with the type and age of relay we were using 
- elaborate more on what a good coordination design is.

- enjoyed this lab, very informative and accurate.

- Good Lab

- Great working with everyone. Happy to be practicing real relay analysis.

- I really enjoyed this lab because actually creating the curves made what the curves actually represent make. more sense

- Maybe some further elaboration on software usage in the lab assignment - overall a good experiment. The only thing difficult was learning ASPEN.

- relay working is good .

- $\quad$ pretty interesting lab

- Very clear on what will I learn in the lab.

Lab 6: Coordination of OC Relays in Radial Systems

- It was nice to see the theoretical relay values matched the physical values for proof of concept.

- elaborate more on what a good coordination design is.

- Very helpful to see how a protection engineer might do "coordination." Would be good for this lab to happen about the same time coordination is discussed in class and preferably before it is tested in a mid-term exam.

- introducing data of phase and ground overcurrent relay elements into the model was somewhat confussing

- This was awesome. Some computer connections to program the relay would have been great :) 
- It would be nice to see how easy the other program works (forgot the name) compared to aspen.

- Relay working is good .

- I wish we had more time on this lab.

- I liked this lab.

- Finally got our hands dirty with the microprocessor!

\section{Lab 7: Digital Relay Radial Over-current Protection}

- Good stuff

- good to know that SEL can accurately provide outputs

- Great hands-on.

- Next year's lab will be great.

- Not fun to program manually but good practice.

- Relay working is good .

- This one took a while to sink in

- Ty, nice.

- We didn't get around to playing with the SEL. However, the new multiamp was great to play with and get to know how it worked. Also, pulling apart the relay to find connections 10-20 was fascinating. I have not had the chance to be really hands-on before so any chance to implement that in the lab is constructive. As a side note: making us look shit up in the manuals is so real-world. Get a better grasp on what is and isn't in there then send the students on hunts. You will be much hated but the skills learned will be immensely useful. blahblahblahhhhhhhh :)

Lab 8: ASPEN looped system protection coordination 
- $\quad$ Have a good break =)

- I found this lab particularly effective in clearing up misconceptions and confusion among myself and team members in regards to looped coordination. The lab was a great experience because it allowed me to familiarize myself with the equipment but also gave us time to come together as a group of students and clarify topics discuss that we found very confusing in the lecture.

- I like building on the labs from before.

- It was nice to have a fresh one liner to start with so we were are in the same place. We could concentrate on the lab instead of trying to backtrack and figure out what was wrong with our original one liner.

- limited to the capabilites of aspen would be nice to have another program that can do the same a little better.

- N/A

- $\quad$ nothing to add

- Our team had problem coordinating the looped system. One relay closest to the bus fault didn't see the fault.

- Thanks!

- The software knowledge is really good for future.

\section{Lab 9: Directional overcurrent testing}

- Good exercise.

- $\quad$ i like this last lab.

- It is really helpful for student like me to understand the working.

- Mapping out the relay lines is a lab within itself. If you ever need any help doing so let me know. 
- N/A

- Taking the relay apart was extremely helpful for me as I do not have much hands-on-inthe-garage-with-my-dad experience. Also, getting tossed into the deep end with the Pulsar and only the manual was so close to being in a real-world type setting I though I should be getting paid!

- thanks.

- Would have been nice to see the rest of the [EM] relays tested, but still was a nice lab. Overall Lab survey comments:

- I think that you know the bugs that need to be worked out for next year's lab. Good luck on your thesis. Too bad I will not have you for any other labs.

- Lab Report Instructions: -proofread ahead of time -pages need to be numbered Lab: -needs to be more organized -would have liked to have an intro to each of the labs that explained the equipment and purpose of the lab

- One of the my favorite lab class from this University. Thanks Jan

- $\quad$ beneficial $=$ )

- I am sure with some reworking based on the feedback you receive, these lab can become an essential addition to the curriculum for this class. Some were a little hard to follow, especially when the equipment wasn't working quite as expected.

- It should be for one credit because it has lot of work to do so better to keep 1 credit for this subject.

- Lab instructions needs improvement in technical writing.

- Thank you Jen!

- Thanks for all of the guidance! The testing with the analog testing equipment was kind of rough at first, but I think that was mostly from lack of experience with reading technical 
documents. I found that the coordination of a looped system could have used a bit more explanation - the topic in itself is a bit confusing, in my opinion, and we did not really go much in depth in class. Other than that, it was great to see, touch, and work with components (EM/SEL relays) in person.

- The lab was very fun and informative. I thought that having to identify the different parts of the relays was useful. The lab testing the melting point of the wires was fun and helped me visualize what we were learning in class. The only thing I didn't like was the number of labs- 9 labs is kind of a lot. I know some of them were supposed to be pretty short, but when you're not familiar with the equipment even a simple lab can take a long time.

- The labs definitely improved as the term went on. Thank you.

\section{Lab course as a separate, one credit course:}

- $\quad \mathrm{i}$ think so. It's so interesting to miss.

- I think that it is important students have a typical understanding of all aspects of protection including use of common software packages. However, including this lab into the course grading may be a better option than making it a seperate grade than the class. Group work does not always reflect on everyones understanding though and presenting it seperately offers a presentation of this fact in transcripts.

- I think the best thing to do would be to include the lab as a percentage of the grade of the class. An extra credit means we have to pay more.

- It makes no difference as long as one goes to school to actually learn

- yes

- Yes

- Yes . 
- Yes! This course was helpful, but the lack of coordination between lecture and lab experiments made it a bit difficult as to "why" we were doing some things.

- Yes, definitely!!

- Yes, this class could benefit from adding the lab. 